\title{
Asymmetric conjugate addition of ethylene sulfonyl fluorides to 3-amido-2-oxindoles: Synthesis of chiral spirocyclic oxindole sultams
}

\author{
Jie Chen, Bao-qin Huang, Zeng-qing Wang, Xue-jing Zhang, Ming Yan* \\ Guangdong Provincial Key Laboratory of Chiral Molecules and Drug Discovery, School of \\ Pharmaceutical Sciences, \\ Sun Yat-sen University, Guangzhou 510006, China. \\ E-mail: yanming@mail.sysu.edu.cn
}

\section{Supporting Information}

\section{Table of Contents}

\begin{tabular}{|l|l|}
\hline 1. General information & $\mathrm{S} 2$ \\
\hline 2. Preparation of 3-aminooxindoles 1a-1y & $\mathrm{S} 2$ \\
\hline 3. Typical procedures and characterization data for products 3a-3y & $\mathrm{S} 13$ \\
\hline 4. Typical procedures and characterization data for products 5 & $\mathrm{S} 45$ \\
\hline 5. Typical procedures and characterization data for products 6ab-6ai & $\mathrm{S} 49$ \\
\hline 6. Procedure for the gram-scale synthesis of 3a & $\mathrm{S} 59$ \\
\hline 7. Procedures for the transformations of 3a & $\mathrm{S} 60$ \\
\hline 8. The X-ray crystal data of products 3v and $\mathbf{6 a b}$ & $\mathrm{S} 62$ \\
\hline 9. $\quad$ References & $\mathrm{S} 64$ \\
\hline 10. NMR spectra & $\mathrm{S} 65$ \\
\hline
\end{tabular}




\section{General information}

${ }^{1} \mathrm{H}$ NMR and ${ }^{13} \mathrm{C}$ NMR spectra were recorded on Bruker $400 \mathrm{MHz}$ or $500 \mathrm{MHz}$ spectrometer in $\mathrm{CDCl}_{3}$ or $\mathrm{DMSO}-d_{6}$ with tetramethylsilane (TMS) as the internal standard. Melting points were measured on a WRS-2A melting point apparatus and are uncorrected. Chemical shifts of protons are reported in parts per million downfield from tetramethylsilane and are referenced to residual protium in the NMR solvent $\left(\mathrm{CDCl}_{3}: \delta 7.26\right.$; DMSO- $\left.d_{6}: \delta 2.50\right)$. Chemical shifts of carbon are referenced to the carbon resonances of the solvent $\left(\mathrm{CDCl}_{3}: \delta 77.0 ; \mathrm{DMSO}-d_{6}: \delta 39.5\right)$. Peaks are labeled as singlet (s), broad singlet (br), doublet (d), triplet (t), double doublet (dd), multiplet (m). The enantiomeric excesses were determined by HPLC with Daicel CHIRALCEL, CHIRALPAK and Nu-Analytical HPLC columns. The chiral HPLC methods were calibrated with the corresponding racemic mixtures. All products were further characterized by HRMS (high resolution mass spectra). Copies of their ${ }^{1} \mathrm{H}$ NMR and ${ }^{13} \mathrm{C}$ NMR spectra were provided.

All reagents were used as received from commercial suppliers without further purification unless otherwise noted. All solvents were dried and distilled prior to use according to the standard protocols. $N$-Bn-isatin oximes ${ }^{1}$, ethenesulfonyl fluoride ${ }^{2}, \beta$-arylethenesulfonyl fluorides ${ }^{3-4}$ and organocatalysts $\mathbf{4 b}-\mathbf{4} \mathbf{i}^{5-9}$ were synthesized in accordance with the reported procedures.

\section{Preparation of 3-amidooxindoles (1a-1y)}

\subsection{Typical procedures for the synthesis of substrates 1a-1q}

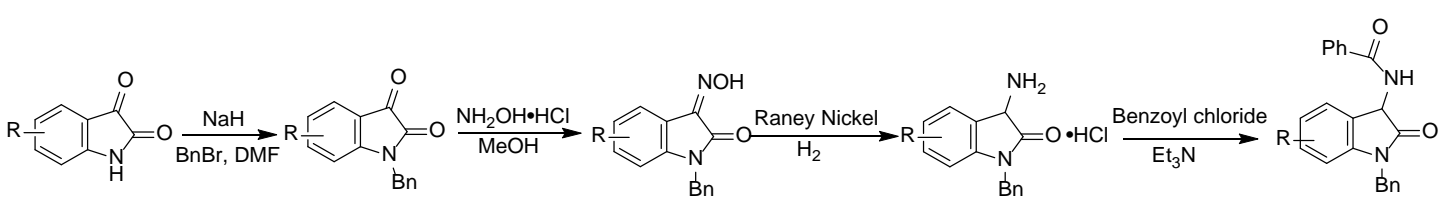

A solution of isatin $(11 \mathrm{mmol})$ in DMF $(30 \mathrm{~mL})$ was cooled under an ice bath. $\mathrm{NaH}$ (60\% dispersion in mineral oil, $12 \mathrm{mmol}$ ) was added portionwise. The color of solution changed from orange to deep purple. When the gas evolution stopped, benzyl bromide $(13 \mathrm{mmol})$ was added slowly, whereupon the mixture was turned red-brown. After the reaction mixture was stirred for $15 \mathrm{~min}$ at room temperature, $\mathrm{H}_{2} \mathrm{O}(100 \mathrm{~mL})$ was added to precipitate $N$-Bn-isatin.

To a solution of $\mathrm{N}$-Bn isatin $(5 \mathrm{mmol})$ in $\mathrm{MeOH}(100 \mathrm{~mL})$ was added hydroxylamine hydrochloride $(6.0 \mathrm{mmol})$ and potassium carbonate $(6.0 \mathrm{mmol})$. The resulting mixture was stirred under reflux overnight. The reaction mixture was concentrated under reduced pressure, and the residue was purified by column chromatography on silica gel (pentane/EtOAc $=2: 1$ ) to give isatin oxime as a yellow solid. 
To a suspension of isatin oxime $(5 \mathrm{mmol})$ in $\mathrm{MeOH}(200 \mathrm{~mL})$ was added Raney Nickel at room temperature in $100 \mathrm{~mL}$ high pressure reactor. The reaction mixture was stirred under $\mathrm{H}_{2}$ atmosphere (8 atm) for $10 \mathrm{~h}$. Then, concentrated $\mathrm{HCl}(20 \mathrm{~mL})$ was added to the mixture. After Raney Nickel was removed by the filtration, the filtrate was concentrated under reduced pressure. The crude product was purified by washing with dichloromethane $(100 \mathrm{~mL})$ to provide 3-amino-oxindole hydrochloride as a white solid.

To a solution of 3-amino-oxindole hydrochloride (2.5 mmol) in $\mathrm{CH}_{2} \mathrm{Cl}_{2}(20 \mathrm{~mL})$ was added $\mathrm{Et}_{3} \mathrm{~N}$ (0.55 mL, $3.75 \mathrm{mmol}$ ) at $0{ }^{\circ} \mathrm{C}$ under $\mathrm{N}_{2}$ atmosphere. The resulting mixture was vigorously stirred for $15 \mathrm{~min}$, and then benzoyl chloride $(2.75 \mathrm{mmol})$ was added slowly via a syringe. The mixture was stirred for $1 \mathrm{~h}$ and concentrated. The residue was purified by flash chromatography to furnish the product as a white solid.

\subsection{Typical procedures for the synthesis of substrates $1 r-1 y$}<smiles>[R]C(=O)NC1C(=O)N(Cc2ccccc2)c2ccc([GeH])cc21</smiles>

To a solution of 3-amino-oxindole hydrochloride $(2.5 \mathrm{mmol})$ in $\mathrm{CH}_{2} \mathrm{Cl}_{2}(20 \mathrm{~mL})$ was added $\mathrm{Et}_{3} \mathrm{~N}(0.55$ $\mathrm{mL}, 3.75 \mathrm{mmol}$ ) at $0{ }^{\circ} \mathrm{C}$ under $\mathrm{N}_{2}$ atmosphere. The resulting mixture was vigorously stirred for $15 \mathrm{~min}$, and then acyl chloride (2.75 mmol) was added slowly via a syringe. The mixture was stirred for $1 \mathrm{~h}$ and concentrated. The residue was purified by flash chromatography to furnish the product as a white solid.

\subsection{Characterizations of substrates 1a-1y}

\section{N-(1-benzyl-2-oxoindolin-3-yl)benzamide (1a)}<smiles>O=C(NC1C(=O)N(Cc2ccccc2)c2ccccc21)c1ccccc1</smiles>

This compound was prepared according to typical procedure 2.1. Petroleum ether / ethyl acetate $=5: 1$ was used as the eluent for column chromatography. White solid (684 mg, 80\%); m.p. 199.7-200.6 ${ }^{\circ} \mathrm{C}$; ${ }^{1} \mathbf{H}$ NMR (500 MHz, $\left.\mathrm{CDCl}_{3}\right) \delta 7.81$ (d, $\left.J=7.5 \mathrm{~Hz}, 2 \mathrm{H}\right), 7.46$ (t, $\left.J=7.5 \mathrm{~Hz}, 1 \mathrm{H}\right), 7.42-7.34$ (m, 6H), 7.347.30 (m, 2H), 7.28-7.23 (m, 1H), 7.18 (t, $J=7.5 \mathrm{~Hz}, 1 \mathrm{H}), 7.01$ (t, $J=7.5 \mathrm{~Hz}, 1 \mathrm{H}), 6.73$ (d, $J=8.0 \mathrm{~Hz}$, 
1H), 5.47 (d, $J=7.0 \mathrm{~Hz}, 1 \mathrm{H}), 4.93$ (dd, $J=63.5,15.5 \mathrm{~Hz}, 2 \mathrm{H}) .{ }^{13} \mathbf{C}$ NMR (126 MHz, $\left.\mathrm{CDCl}_{3}\right) \delta 174.72$, 167.60, 142.95, 135.47, 133.06, 131.92, 129.11, 128.87, 128.53, 127.74, 127.36, 126.61, 124.55, 123.13, 109.41, 53.33, 44.22. HRMS ESI (m/z): Calcd for $\mathrm{C}_{22} \mathrm{H}_{18} \mathrm{~N}_{2} \mathrm{O}_{2},[\mathrm{M}+\mathrm{H}]^{+}$, 341.1269; found: 341.1243 .

N-(2-oxo-1-phenylindolin-3-yl)benzamide (1b)<smiles>O=C(NC1C(=O)N(c2ccccc2)c2ccccc21)c1ccccc1</smiles>

This compound was prepared according to typical procedure 2.1. Petroleum ether $/$ ethyl acetate $=5: 1$ was used as the eluent for column chromatography. White solid (680 mg, 83\%); m.p. 204.5-206.6 ${ }^{\circ} \mathrm{C}$; ${ }^{1} \mathbf{H}$ NMR (400 MHz, DMSO-d $d_{6} \delta 9.57$ (d, $\left.J=7.6 \mathrm{~Hz}, 1 \mathrm{H}\right), 7.93$ (d, $\left.J=7.2 \mathrm{~Hz}, 2 \mathrm{H}\right), 7.63-7.56$ (m , 3H), 7.54-7.46 (m, 5H), 7.32 (d, $J=7.2 \mathrm{~Hz}, 1 \mathrm{H}), 7.26$ (t, $J=7.6 \mathrm{~Hz}, 1 \mathrm{H}), 7.08$ (t, $J=7.2 \mathrm{~Hz}, 1 \mathrm{H}), 6.76$ (d, $J$ $=8.0 \mathrm{~Hz}, 1 \mathrm{H}), 5.46(\mathrm{~d}, J=7.6 \mathrm{~Hz}, 1 \mathrm{H}) .{ }^{13} \mathrm{C}$ NMR (101 MHz, DMSO-d $)_{6} \delta$ 173.94, 166.48, 144.49, 135.23, 133.62, 132.25, 130.07, 129.01, 128.96, 128.42, 127.87, 127.67, 127.15, 124.21, 123.18, 109.19, 53.18. HRMS ESI (m/z): Calcd for $\mathrm{C}_{21} \mathrm{H}_{16} \mathrm{~N}_{2} \mathrm{O}_{2}$, [M+H] $]^{+}$, 329.1285; found: 329.1274.

N-(1-methyl-2-oxoindolin-3-yl)benzamide (1c)<smiles>CN1C(=O)C(NC(=O)c2ccccc2)c2ccccc21</smiles>

This compound was prepared according to typical procedure 2.1. Petroleum ether / ethyl acetate $=5: 1$ was used as the eluent for column chromatography. White solid (532 mg, 80\%); m.p. 190.5-192.3 ${ }^{\circ} \mathrm{C}$; ${ }^{1} \mathbf{H}$ NMR (500 MHz, $\left.\mathrm{CDCl}_{3}\right) \delta 7.81$ (d, $\left.J=7.5 \mathrm{~Hz}, 2 \mathrm{H}\right), 7.51-7.45(\mathrm{~m}, 1 \mathrm{H}), 7.40$ (dd, $J=16.0,8.0 \mathrm{~Hz}$, 3H), 7.35-7.30 (m, 1H), 7.15 (d, $J=7.0 \mathrm{~Hz}, 1 \mathrm{H}), 7.06$ (t, $J=7.5 \mathrm{~Hz}, 1 \mathrm{H}), 6.86$ (d, $J=8.0 \mathrm{~Hz}, 1 \mathrm{H}), 5.49$ (d, $J=7.0 \mathrm{~Hz}, 1 \mathrm{H}), 3.23$ (s, 3H). ${ }^{13} \mathbf{C}$ NMR $\left(101 \mathrm{MHz}, \mathrm{CDCl}_{3}\right) \delta 174.54,167.69,143.77,133.09,131.93$, 129.27, 128.54, 127.28, 126.65, 124.68, 123.16, 108.36, 53.14, 26.56. HRMS ESI (m/z): Calcd for $\mathrm{C}_{16} \mathrm{H}_{14} \mathrm{~N}_{2} \mathrm{O}_{2}$, [M+H] $]^{+}$, 267.1128; found: 267.1127.

N-(1-benzyl-5-fluoro-2-oxoindolin-3-yl)benzamide (1d)<smiles>O=C(NC1C(=O)N(Cc2ccccc2)c2ccc(F)cc21)c1ccccc1</smiles>

This compound was prepared according to typical procedure 2.1. Petroleum ether / ethyl acetate $=5: 1$ was used as the eluent for column chromatography. White solid (720 mg, 80\%); m.p.198.9-201.3 ${ }^{\circ} \mathrm{C} ;{ }^{1} \mathbf{H}$ 
NMR (400 MHz, CDCl $) \delta 7.82(\mathrm{~d}, J=7.6 \mathrm{~Hz}, 2 \mathrm{H}), 7.54-7.49(\mathrm{~m}, 1 \mathrm{H}), 7.45-7.28(\mathrm{~m}, 7 \mathrm{H}), 7.25-7.12$ (m, 2H), 6.89 (td, $J=9.2,2.4 \mathrm{~Hz}, 1 \mathrm{H}$ ), 6.65 (dd, $J=8.8,4.0 \mathrm{~Hz}, 1 \mathrm{H}$ ), 5.43 (d, $J=3.6 \mathrm{~Hz}, 1 \mathrm{H}$ ), 4.96 (dd, $J=32.8,15.6 \mathrm{~Hz}, 2 \mathrm{H}) .{ }^{13} \mathrm{C}$ NMR $\left(101 \mathrm{MHz}, \mathrm{CDCl}_{3}\right) \delta 174.27,167.65,159.46(\mathrm{~d}, J \mathrm{C}-\mathrm{F}=241.8 \mathrm{~Hz})$, 138.81, 135.13, 132.80, 132.16, 128.95, 128.65, 128.13 (d, JC-F $=8.4$ Hz), 127.87, 127.35, 127.31, 115.41 $\left(\mathrm{d}, J_{\mathrm{C}-\mathrm{F}}=23.6 \mathrm{~Hz}\right), 112.85$ (d, $\left.J_{\mathrm{C}-\mathrm{F}}=25.2 \mathrm{~Hz}\right), 110.04$ (d, JC-F $\left.=8.0 \mathrm{~Hz}\right), 53.62,44.42 .{ }^{19}$ F NMR (471 MHz, $\left.\mathrm{CDCl}_{3}\right) \delta$-119.64. HRMS ESI (m/z): Calcd for $\mathrm{C}_{22} \mathrm{H}_{17} \mathrm{~N}_{2} \mathrm{O}_{2} \mathrm{~F},[\mathrm{M}+\mathrm{H}]^{+}$, 361.1347; found: 361.1336.

N-(1-benzyl-5-chloro-2-oxoindolin-3-yl)benzamide (1e)<smiles>O=C(NC1C(=O)N(Cc2ccccc2)c2ccc(Cl)cc21)c1ccccc1</smiles>

This compound was prepared according to typical procedure 2.1. Petroleum ether / ethyl acetate $=5: 1$ was used as the eluent for column chromatography. White solid (760 mg, 81\%); m.p. 225.4-227.5 ${ }^{\circ} \mathrm{C}$; ${ }^{1}$ H NMR (500 MHz, DMSO-d $\left.d_{6}\right) \delta 9.57$ (d, $J=7.5$ Hz, 1H), 7.97-7.89 (m, 2H), 7.61-7.56 (m, 1H), 7.51 (t, $J=7.5 \mathrm{~Hz}, 2 \mathrm{H}$ ), 7.45 (d, $J=7.5 \mathrm{~Hz}, 2 \mathrm{H}$ ), 7.35 (t, $J=7.5 \mathrm{~Hz}, 2 \mathrm{H}), 7.31-7.23$ (m, 3H), 6.81 (d, $J=8.0$ $\mathrm{Hz}, 1 \mathrm{H}$ ), 5.39 (d, $J=7.5 \mathrm{~Hz}, 1 \mathrm{H}$ ), 4.95 (dd, $J=43.0,16.0 \mathrm{~Hz}, 2 \mathrm{H}) .{ }^{13} \mathrm{C}$ NMR (126 MHz, DMSO- $d_{6}$ ) $\delta$ 174.21, 166.49, 142.43, 136.41, 133.50, 132.28, 130.02, 129.00, 128.93, 128.50, 127.96, 127.78, 127.67, 126.78, 123.71, 110.79, 53.29, 43.41. HRMS ESI (m/z): Calcd for $\mathrm{C}_{22} \mathrm{H}_{17} \mathrm{~N}_{2} \mathrm{O}_{2} \mathrm{Cl}$, [M+H]+, 377.1051; found: 377.1048 .

\section{N-(1-benzyl-5-bromo-2-oxoindolin-3-yl)benzamide (1f)}<smiles>CCN1C(=O)C(NC(=O)c2ccccc2)c2cc(Br)ccc21</smiles>

This compound was prepared according to typical procedure 2.1. Petroleum ether / ethyl acetate $=5: 1$ was used as the eluent for column chromatography. White solid (840 mg, 80\%); m.p. 226.4-228.2 ${ }^{\circ} \mathrm{C}$; ${ }^{1}$ H NMR (400 MHz, DMSO-d $\left.d_{6}\right) \delta 9.56(\mathrm{~d}, J=7.6 \mathrm{~Hz}, 1 \mathrm{H}), 7.96$-7.89 (m, 2H), 7.62-7.56 (m, 1H), 7.547.48 (m, 2H), 7.47-7.42( m, 2H), 7.41-7.32 (m, 4H), 7.30-7.25 (m, 1H), 6.80-6.74 (m, 1H), 5.39 (d, $J=$ $7.2 \mathrm{~Hz}, 1 \mathrm{H}), 4.95$ (dd, $J=37.2,16.4 \mathrm{~Hz}, 2 \mathrm{H}) .{ }^{13} \mathrm{C}$ NMR $\left(101 \mathrm{MHz}\right.$, DMSO-d $\left.d_{6}\right) \delta 174.10,166.49,142.84$, 136.38, 133.49, 132.29, 131.37, 130.39, 129.00, 128.94, 127.97, 127.78, 127.66, 126.37, 114.47, 111.34, 53.22, 43.38. HRMS ESI (m/z): Calcd for $\mathrm{C}_{22} \mathrm{H}_{17} \mathrm{~N}_{2} \mathrm{O}_{2} \mathrm{Br}$, [M+H] ${ }^{+}$, 421.0546; found: 421.0545 .

N-(1-benzyl-5-methyl-2-oxoindolin-3-yl) benzamide (1g) 
<smiles>Cc1ccc2c(c1)C(NC(=O)c1ccccc1)C(=O)N2Cc1ccccc1</smiles>

This compound was prepared according to typical procedure 2.1. Petroleum ether $/$ ethyl acetate $=5: 1$ was used as the eluent for column chromatography. White solid (756 mg, 85\%); m.p. 204.9-206.5 ${ }^{\circ} \mathrm{C}$; ${ }^{1}$ H NMR (500 MHz, DMSO-d $\left.d_{6}\right) \delta 9.48$ (d, $J=7.5$ Hz, 1H), 7.94 (d, $J=7.5$ Hz, 2H), 7.61-7.55 (m, 1H), 7.51 (t, $J=7.5 \mathrm{~Hz}, 2 \mathrm{H}), 7.44$ (d, $J=7.5 \mathrm{~Hz}, 2 \mathrm{H}), 7.34$ (t, $J=7.5 \mathrm{~Hz}, 2 \mathrm{H}), 7.29-7.24(\mathrm{~m}, 1 \mathrm{H}), 7.06$ (s, $1 \mathrm{H}$ ), 6.99 (d, $J=8.0 \mathrm{~Hz}, 1 \mathrm{H}), 6.68$ (d, $J=8.0 \mathrm{~Hz}, 1 \mathrm{H}), 5.39$ (d, $J=7.5 \mathrm{~Hz}, 1 \mathrm{H}), 4.92$ (dd, $J=39.0,16.0 \mathrm{~Hz}$, 2H), 2.22 (s, 3H). ${ }^{13} \mathrm{C}$ NMR (126 MHz, DMSO-d $) \delta 174.36,166.42,141.13,136.81,133.75,132.17$, 131.71, 128.94, 128.91, 127.92, 127.79, 127.69, 124.43, 109.22, 53.13, 43.36, 21.01. HRMS ESI (m/z): Calcd for $\mathrm{C}_{23} \mathrm{H}_{20} \mathrm{~N}_{2} \mathrm{O}_{2},[\mathrm{M}+\mathrm{H}]^{+}$, 357.1598; found: 357.1586.

\section{N-(1-benzyl-5-methoxy-2-oxoindolin-3-yl)benzamide (1h)}<smiles>COc1ccc2c(c1)C(NC(=O)c1ccccc1)C(=O)N2Cc1ccccc1</smiles>

This compound was prepared according to typical procedure 2.1. Petroleum ether / ethyl acetate $=5: 1$ was used as the eluent for column chromatography. White solid (790 mg, 85\%); m.p. 201.4-202.9 ${ }^{\circ} \mathrm{C}$; ${ }^{1}$ H NMR (500 MHz, DMSO- $\left.d_{6}\right) \delta 9.48$ (d, $\left.J=8.0 \mathrm{~Hz}, 1 \mathrm{H}\right), 7.93$ (d, $\left.J=7.5 \mathrm{~Hz}, 2 \mathrm{H}\right), 7.61-7.55(\mathrm{~m}, 1 \mathrm{H})$, 7.53-7.48 (m, 2H), 7.44 (d, $J=7.0 \mathrm{~Hz}, 2 \mathrm{H}), 7.34$ (t, $J=7.5 \mathrm{~Hz}, 2 \mathrm{H}), 7.29-7.24$ (m, 1H), 6.86 (s, 1H), 6.77 (dd, $J=8.5,2.5 \mathrm{~Hz}, 1 \mathrm{H}$ ), 6.70 (d, $J=8.5 \mathrm{~Hz}, 1 \mathrm{H}$ ), 5.39 (d, $J=8.0 \mathrm{~Hz}, 1 \mathrm{H}$ ), 4.91 (dd, $J=39.5,16.0$ $\mathrm{Hz}, 2 \mathrm{H}), 3.66$ (s, 3H). ${ }^{13} \mathrm{C}$ NMR (126 MHz, DMSO-d $) \delta 174.13,166.47,155.92,136.87,136.82,133.76$, 132.17, 129.07, 128.95, 128.90, 127.94, 127.72, 127.68, 113.22, 110.93, 109.89, 55.98, 53.44, 43.40. HRMS ESI (m/z): Calcd for $\mathrm{C}_{23} \mathrm{H}_{20} \mathrm{~N}_{2} \mathrm{O}_{3}$, [M+H] $]^{+}$, 373.1547; found: 373.1536.

\section{N-(1-benzyl-6-fluoro-2-oxoindolin-3-yl)benzamide (1i)}<smiles>O=C(NC1C(=O)N(Cc2ccccc2)c2cc(F)ccc21)c1ccccc1</smiles>

$1 \mathrm{i}$

This compound was prepared according to typical procedure 2.1. Petroleum ether / ethyl acetate $=5: 1$ was used as the eluent for column chromatography. White solid (747 mg, 83\%); m.p. 202-203.5 ${ }^{\circ} \mathrm{C} ;{ }^{1} \mathbf{H}$ NMR (500 MHz, DMSO-d $)) \delta .51(\mathrm{~d}, J=7.5 \mathrm{~Hz}, 1 \mathrm{H}), 7.92$ (d, $J=7.5 \mathrm{~Hz}, 2 \mathrm{H}), 7.58$ (t, $J=7.5 \mathrm{~Hz}$, 1H), 7.53-7.45 (m, 4H), 7.36 (t, $J=7.5 \mathrm{~Hz}, 2 \mathrm{H}), 7.31-7.22(\mathrm{~m}, 2 \mathrm{H}), 6.82-6.73$ (m, 2H), 5.36 (d, $J=7.5$ 
Hz, 1H), 4.95 (dd, $J=37.5,15.5 \mathrm{~Hz}, 2 \mathrm{H}) .{ }^{13} \mathrm{C}$ NMR (126 MHz, DMSO-d $\left.)_{6}\right) \delta 174.90,166.44,162.93$ (d, $\left.J_{\mathrm{C}-\mathrm{F}}=241.9 \mathrm{~Hz}\right), 145.29\left(\mathrm{~d}, J_{\mathrm{C}-\mathrm{F}}=12.0 \mathrm{~Hz}\right), 136.45,133.62,132.23,129.00,128.93,127.90,127.83$, $124.97\left(\mathrm{~d}, J_{\mathrm{C}-\mathrm{F}}=9.8 \mathrm{~Hz}\right), 123.56$ (d, JC-F $=2.4 \mathrm{~Hz}$ ), 108.43 (d, JC-F $\left.=22.1 \mathrm{~Hz}\right), 98.05$ (d, JC-F = 27.8 Hz), 52.74, 43.44. ${ }^{19}$ F NMR (471 MHz, DMSO- $\left.d_{6}\right) \delta-112.60$. HRMS ESI (m/z): Calcd for $\mathrm{C}_{22} \mathrm{H}_{17} \mathrm{~N}_{2} \mathrm{O}_{2} \mathrm{~F}$, $[\mathrm{M}+\mathrm{H}]^{+}, 361.1347$; found: 361.1344.

N-(1-benzyl-6-chloro-2-oxoindolin-3-yl)benzamide (1j)<smiles>O=C(NC1C(=O)N(Cc2ccccc2)c2cc(Cl)ccc21)c1ccccc1</smiles>

1j

This compound was prepared according to typical procedure 2.1. Petroleum ether / ethyl acetate $=5: 1$ was used as the eluent for column chromatography. White solid (799 mg, 85\%); m.p. 216.6-218.5 ${ }^{\circ} \mathrm{C}$; ${ }^{1}$ H NMR (500 MHz, DMSO- $d_{6}$ ) $\delta 9.57$ (d, $J=7.5$ Hz, 1H), 7.94-7.89 (m, 2H), 7.61-7.55 (m, 1H), 7.537.45 (m, 4H), 7.36 (t, $J=7.5 \mathrm{~Hz}, 2 \mathrm{H}), 7.30-7.23$ (m, 2H), 7.03 (dd, $J=8.0,1.5 \mathrm{~Hz}, 1 \mathrm{H}), 6.92$ (d, $J=1.5$ $\mathrm{Hz}, 1 \mathrm{H}), 5.35$ (d, $J=7.5 \mathrm{~Hz}, 1 \mathrm{H}), 4.97$ (dd, $J=39.5,16.0 \mathrm{~Hz}, 2 \mathrm{H}) .{ }^{13} \mathrm{C}$ NMR $\left(126 \mathrm{MHz}\right.$, DMSO- $\left.d_{6}\right) \delta$ 174.61, 166.43, 145.07, 136.41, 133.51, 133.13, 132.27, 129.01, 128.94, 127.91, 127.81, 127.71, 126.77, 125.00, 122.22, 109.61, 52.90, 43.33. HRMS ESI (m/z): Calcd for $\mathrm{C}_{22} \mathrm{H}_{17} \mathrm{~N}_{2} \mathrm{O}_{2} \mathrm{Cl},[\mathrm{M}+\mathrm{H}]^{+}, 377.1051$; found: 377.1046 .

N-(1-benzyl-6-bromo-2-oxoindolin-3-yl)benzamide (1k)<smiles>O=C(NC1C(=O)N(Cc2ccccc2)c2cc(Br)ccc21)c1ccccc1</smiles>

This compound was prepared according to typical procedure 2.1. Petroleum ether $/$ ethyl acetate $=5: 1$ was used as the eluent for column chromatography. White solid (892 mg, 85\%); m.p. 215.6-217.1 ${ }^{\circ} \mathrm{C}$; ${ }^{1} \mathbf{H}$ NMR (400 MHz, DMSO-d $\left.d_{6}\right) \delta 9.53(\mathrm{dd}, J=22.4,7.6 \mathrm{~Hz}, 1 \mathrm{H}), 7.99-7.84(\mathrm{~m}, 2 \mathrm{H}), 7.62-7.43(\mathrm{~m}, 5 \mathrm{H})$, 7.40-7.15 (m, 5H), 7.07-6.96 (m, 1H), 5.37 (dd, $J=37.6,7.6 \mathrm{~Hz}, 1 \mathrm{H}), 5.05-4.86(\mathrm{~m}, 2 \mathrm{H}) .{ }^{13} \mathrm{C}$ NMR $(101$ MHz, DMSO- $\left.d_{6}\right) \delta 174.50,166.44,145.21,136.41,133.50,132.28,129.01,128.95,127.90,127.81$, 127.68, 127.23, 125.38, 125.17, 121.37, 112.29, 52.96, 43.30. HRMS ESI (m/z): Calcd for $\mathrm{C}_{22} \mathrm{H}_{17} \mathrm{~N}_{2} \mathrm{O}_{2} \mathrm{Br},[\mathrm{M}+\mathrm{H}]^{+}$, 421.0546; found: 421.0549 .

\section{N-(1-benzyl-6-methoxy-2-oxoindolin-3-yl)benzamide (11)}


<smiles>COc1ccc2c(c1)N(Cc1ccccc1)C(=O)C2NC(=O)c1ccccc1</smiles>

11

This compound was prepared according to typical procedure 2.1. Petroleum ether $/$ ethyl acetate $=5: 1$ was used as the eluent for column chromatography. White solid (772 mg, 83\%); m.p. 201.4-203.2 ${ }^{\circ} \mathrm{C}$; ${ }^{1} \mathbf{H}$ NMR (400 MHz, $\left.\mathrm{CDCl}_{3}\right) \delta 7.83(\mathrm{~d}, J=7.2 \mathrm{~Hz}, 2 \mathrm{H}), 7.53-7.47(\mathrm{~m}, 1 \mathrm{H}), 7.41(\mathrm{t}, J=7.6 \mathrm{~Hz}, 2 \mathrm{H}), 7.38-$ 7.27 (m, 6H), 7.02 (d, $J=7.2 \mathrm{~Hz}, 1 \mathrm{H}), 6.51$ (dd, $J=8.4,2.4 \mathrm{~Hz}, 1 \mathrm{H}), 6.33$ (d, $J=2.0 \mathrm{~Hz}, 1 \mathrm{H}$ ), 5.51 (d, $J=7.2 \mathrm{~Hz}, 1 \mathrm{H}), 4.92$ (dd, $J=50.4,15.6 \mathrm{~Hz}, 2 \mathrm{H}), 3.72(\mathrm{~s}, 3 \mathrm{H}) .{ }^{13} \mathbf{C}$ NMR $\left(101 \mathrm{MHz}, \mathrm{CDCl}_{3}\right) \delta 175.02$, 167.65, 160.80, 144.32, 135.41, 133.25, 131.93, 128.87, 128.56, 127.77, 127.40, 127.29, 125.51, 118.44, 106.56, 97.71, 55.47, 52.77, 44.25. HRMS ESI (m/z): Calcd for $\mathrm{C}_{23} \mathrm{H}_{20} \mathrm{~N}_{2} \mathrm{O}_{3},[\mathrm{M}+\mathrm{H}]^{+}$, 373.1547; found: 373.1536.

\section{N-(1-benzyl-7-fluoro-2-oxoindolin-3-yl)benzamide (1m)}<smiles>O=C(NC1C(=O)N(Cc2ccccc2)c2c(F)cccc21)c1ccccc1</smiles>

This compound was prepared according to typical procedure 2.1. Petroleum ether $/$ ethyl acetate $=5: 1$ was used as the eluent for column chromatography. White solid (765 mg, 85\%); m.p.184.6-186.3 ${ }^{\circ} \mathrm{C} ;{ }^{1} \mathbf{H}$ NMR (500 MHz, DMSO-d $) \delta 9.61$ (d, $J=7.5$ Hz, 1H), 7.96 -7.91 (m, 2H), 7.60-7.56 (m, 1H), 7.537.49 (m, 2H), 7.42 (d, $J=7.5$ Hz, 2H), 7.37-7.24 (m, 4H), 7.12-7.06 (m, 2H), 7.04 -6.97 (m, 1H), 5.43 (d, $J=7.5 \mathrm{~Hz}, 1 \mathrm{H}), 5.03$ (dd, $J=50.0,16.0 \mathrm{~Hz}, 2 \mathrm{H}) .{ }^{13} \mathrm{C}$ NMR (126 MHz, DMSO-d $)$ ) $\delta 174.35,166.46$, $147.06\left(\mathrm{~d}, J_{\mathrm{C}-\mathrm{F}}=242.3 \mathrm{~Hz}\right), 137.71,133.50,132.30,131.06\left(\mathrm{~d}, J_{\mathrm{C}-\mathrm{F}}=3.0 \mathrm{~Hz}\right), 129.86\left(\mathrm{~d}, J_{\mathrm{C}-\mathrm{F}}=8.8 \mathrm{~Hz}\right)$, 128.95, 128.88, 127.92, 127.58, 127.07, 123.68 (d, JC-F $=6.3 \mathrm{~Hz}), 119.92$ (d, JC-F $=2.1 \mathrm{~Hz}), 116.81$ (d, $\left.J_{\mathrm{C}-\mathrm{F}}=19.2 \mathrm{~Hz}\right), 53.43,45.19,45.16 .{ }^{19}$ F NMR (471 MHz, DMSO- $\left.d_{6}\right) \delta-135.74$. HRMS ESI (m/z): Calcd for $\mathrm{C}_{22} \mathrm{H}_{17} \mathrm{~N}_{2} \mathrm{O}_{2} \mathrm{~F}$, [M+H] $]^{+}$, 361.1347; found: 361.1341.

\section{N-(1-benzyl-7-chloro-2-oxoindolin-3-yl)benzamide (1n)}


<smiles>O=C(NC1C(=O)N(Cc2ccccc2)c2c(Cl)cccc21)c1ccccc1</smiles>

This compound was prepared according to typical procedure 2.1. Petroleum ether $/$ ethyl acetate $=5: 1$ was used as the eluent for column chromatography. White solid (752 mg, 80\%); m.p. 222.8-223.4 ${ }^{\circ} \mathrm{C}$; ${ }^{1}$ H NMR (500 MHz, DMSO- $d_{6}$ ) $\delta 9.63$ (d, $J=7.5$ Hz, 1H), 7.96-7.89 (m, 2H), 7.61-7.56 (m, 1H), 7.547.48 (m, 2H), 7.39 (d, $J=7.5 \mathrm{~Hz}, 2 \mathrm{H}), 7.36-7.3(\mathrm{~m}, 2 \mathrm{H}), 7.28-7.19$ (m, 3H), 7.02 (dd, $J=8.0,7.5 \mathrm{~Hz}$, 1H), 5.42 (d, $J=7.5 \mathrm{~Hz}, 1 \mathrm{H}$ ), 5.27 (dd, $J=34.5,17.0 \mathrm{~Hz}, 2 \mathrm{H}) .{ }^{13} \mathbf{C}$ NMR (126 MHz, DMSO-d $\left.)\right) \delta 175.18$, 166.41, 139.34, 138.40, 133.44, 132.33, 131.23, 130.89, 128.97, 128.83, 127.92, 127.25, 126.58, 124.09, 122.58, 114.36, 53.11, 44.87. HRMS ESI (m/z): Calcd for $\mathrm{C}_{22} \mathrm{H}_{17} \mathrm{~N}_{2} \mathrm{O}_{2} \mathrm{Cl}$, $[\mathrm{M}+\mathrm{H}]^{+}, 377.1051$; found: 377.1049 .

\section{N-(1-benzyl-7-bromo-2-oxoindolin-3-yl)benzamide (10)}<smiles>O=C(NC1C(=O)N(Cc2ccccc2)c2c(Br)cccc21)c1ccccc1</smiles>

This compound was prepared according to typical procedure 2.1. Petroleum ether $/$ ethyl acetate $=5: 1$ was used as the eluent for column chromatography. White solid (840 mg, 80\%); m.p. 209.6-211.8 ${ }^{\circ} \mathrm{C}$; ${ }^{1} \mathbf{H}$ NMR (400 MHz, DMSO- $d_{6}$ ) $\delta 9.63$ (d, $\left.J=7.2 \mathrm{~Hz}, 1 \mathrm{H}\right), 7.95-7.90$ (m, 2H), $7.60-7.57$ (m, 1H), 7.547.49 (m, 2H), 7.41-7.37 (m, 3H), 7.34 (t, $J=7.5 \mathrm{~Hz}, 2 \mathrm{H}), 7.29-7.24$ (m, 2H), 6.96 (t, $J=7.7 \mathrm{~Hz}, 1 \mathrm{H})$, $5.43(\mathrm{~d}, J=7.3 \mathrm{~Hz}, 1 \mathrm{H}), 5.32$ (dd, $J=24.8,17.2 \mathrm{~Hz}, 2 \mathrm{H}) .{ }^{13} \mathrm{C}$ NMR (101 MHz, DMSO-d 6 ) $\delta 175.37$, 166.41, 140.76, 138.32, 134.25, 133.44, 132.33, 131.57, 128.97, 128.80, 127.91, 127.19, 126.59, 124.49, 123.04, 101.80, 53.06, 44.60. HRMS ESI (m/z): Calcd for $\mathrm{C}_{22} \mathrm{H}_{17} \mathrm{~N}_{2} \mathrm{O}_{2} \mathrm{Br}$, $[\mathrm{M}+\mathrm{H}]^{+}$, 421.0546; found: 421.0550 .

N-(1-benzyl-2-oxo-7-(trifluoromethyl)indolin-3-yl)benzamide (1p)<smiles>O=C(NC1C(=O)N(Cc2ccccc2)c2c1cccc2C(F)(F)F)c1ccccc1</smiles>

This compound was prepared according to typical procedure 2.1. Petroleum ether $/$ ethyl acetate $=5: 1$ was used as the eluent for column chromatography. White solid (768 mg, 75\%); m.p. 183.5-186.4 ${ }^{\circ} \mathrm{C}$; ${ }^{1} \mathbf{H}$ NMR (400 MHz, $\left.\mathrm{CDCl}_{3}\right) \delta 7.80(\mathrm{~d}, J=7.6 \mathrm{~Hz}, 1 \mathrm{H}), 7.70(\mathrm{~d}, J=7.6 \mathrm{~Hz}, 2 \mathrm{H}), 7.56(\mathrm{~d}, J=8.4 \mathrm{~Hz}$, 1H), 7.51 (d, $J=7.6 \mathrm{~Hz}, 1 \mathrm{H}), 7.42$ (t, $J=7.6 \mathrm{~Hz}, 1 \mathrm{H}), 7.31-7.21(\mathrm{~m}, 6 \mathrm{H}), 7.20-7.15$ (m, $1 \mathrm{H}), 7.11$ (t, $J$ 
$=7.6 \mathrm{~Hz}, 1 \mathrm{H}), 5.22(\mathrm{~s}, 2 \mathrm{H}), 5.20(\mathrm{~s}, 1 \mathrm{H}) .{ }^{13} \mathrm{C}$ NMR $\left(101 \mathrm{MHz}, \mathrm{CDCl}_{3}\right) \delta$ 176.42, 167.37, 140.82, 136.06, 132.31, 132.02, 129.47, 128.50, 128.44, 127.85, 127.29, 127.14 (d, JC-F $=6.2 \mathrm{~Hz}$ ), 126.96, 125.80, 122.76, $113.15\left(\mathrm{~d}, \mathrm{JC}_{\mathrm{C}-\mathrm{F}}=32.9 \mathrm{~Hz}\right), 52.14,46.17 .{ }^{19} \mathbf{F}$ NMR $\left(376 \mathrm{MHz}, \mathrm{CDCl}_{3}\right) \delta$-54.72. HRMS ESI (m/z): Calcd for $\mathrm{C}_{23} \mathrm{H}_{17} \mathrm{~N}_{2} \mathrm{O}_{2} \mathrm{~F}_{3},[\mathrm{M}+\mathrm{H}]^{+}$, 411.1315; found: 411.1313.

N-(1-benzyl-5,7-dimethyl-2-oxoindolin-3-yl)benzamide (1q)<smiles>Cc1cc(C)c2c(c1)C(NC(=O)c1ccccc1)C(=O)N2Cc1ccccc1</smiles>

This compound was prepared according to typical procedure 2.1. Petroleum ether / ethyl acetate $=5: 1$ was used as the eluent for column chromatography. White solid (749 mg, 81\%); m.p. 230.1-232.5 ${ }^{\circ} \mathrm{C}$; ${ }^{1} \mathbf{H}$ NMR $\left(400 \mathrm{MHz}, \mathrm{CDCl}_{3}\right) \delta 7.82(\mathrm{~d}, J=7.6 \mathrm{~Hz}, 2 \mathrm{H}), 7.47$ (t, $\left.J=7.2 \mathrm{~Hz}, 1 \mathrm{H}\right), 7.41-7.30$ (m, 5H), 7.267.22 (m, 2H), 7.08 (s, 1H), 6.78 (s, 1H), 5.46 (d, $J=7.2 \mathrm{~Hz}, 1 \mathrm{H}), 5.27-5.12$ (m, 2H), 2.22 (d, $J=3.2 \mathrm{~Hz}$, 6H). ${ }^{13}$ C NMR (101 MHz, CDCl $) \delta 175.61,167.53,138.43,137.39,133.51,133.12,132.85,131.87$, 128.92, 128.51, 127.43, 127.37, 127.24, 125.76, 123.15, 119.82, 53.08, 45.44, 20.69, 18.54. HRMS ESI (m/z): Calcd for $\mathrm{C}_{24} \mathrm{H}_{22} \mathrm{~N}_{2} \mathrm{O}_{2}$, [M+H] $]^{+}$, 371.1754; found: 371.1745 .

N-(1-benzyl-2-oxoindolin-3-yl)-2-naphthamide (1r)<smiles></smiles>

This compound was prepared according to typical procedure 2.2. Petroleum ether / ethyl acetate $=4: 1$ was used as the eluent for column chromatography. White solid (755 mg, 76\%); m.p. 190.4-191.7 ${ }^{\circ} \mathrm{C}$; ${ }^{1}$ H NMR (400 MHz, DMSO-d $\left.d_{6}\right) \delta 9.67$ (d, $J=7.6 \mathrm{~Hz}, 1 \mathrm{H}$ ), 8.55 (s, 1H), 8.06-7.97 (m, 4H), 7.67-7.59 (m, 2H), 7.48 (d, $J=7.2 \mathrm{~Hz}, 2 \mathrm{H}$ ), 7.39-7.33 (m, 2H), 7.31-7.25 (m, 2H), 7.24-7.18 m, 1H), 7.05-6.97(m , 1H), 6.82 (d, $J=7.6 \mathrm{~Hz}, 1 \mathrm{H}), 5.50$ (d, $J=7.6 \mathrm{~Hz}, 1 \mathrm{H}), 4.96$ (dd, $J=34.8,16.0 \mathrm{~Hz}, 2 \mathrm{H}) .{ }^{13} \mathbf{C}$ NMR $(101$ MHz, DMSO- $\left.d_{6}\right) \delta 174.51,166.59,143.57,136.75,134.79,132.59,131.14,129.41,128.99,128.86$, 128.54, 128.41, 128.34, 128.15, 127.79, 127.74, 127.36, 124.59, 123.83, 122.70, 109.48, 53.18, 43.38. HRMS ESI (m/z): Calcd for $\mathrm{C}_{26} \mathrm{H}_{20} \mathrm{~N}_{2} \mathrm{O}_{2}$, [M+H] $]^{+}$, 393.1598; found:393.1599.

N-(1-benzyl-2-oxoindolin-3-yl)-1H-indole-3-carboxamide (1s) 
<smiles>O=C(NC1C(=O)N(Cc2ccccc2)c2ccccc21)c1c[nH]c2ccccc12</smiles>

This compound was prepared according to typical procedure 2.2. Petroleum ether $/$ ethyl acetate $=4: 1$ was used as the eluent for column chromatography. White solid (695 mg, 73\%); m.p. 185.5-186.4 ${ }^{\circ} \mathrm{C}$; ${ }^{1} \mathbf{H}$ NMR (500 MHz, $\left.\mathrm{CDCl}_{3}\right) \delta 9.27(\mathrm{~s}, 1 \mathrm{H}), 8.07$ (d, $\left.J=7.5 \mathrm{~Hz}, 1 \mathrm{H}\right), 7.46$ (s, 1H), 7.35 (d, $J=7.6 \mathrm{~Hz}$, 2H), 7.28 (t, $J=6.8 \mathrm{~Hz}, 3 \mathrm{H}), 7.25-7.20$ (m, 1H), 7.17 (d, $J=7.8 \mathrm{~Hz}, 2 \mathrm{H}), 7.15-7.05$ (m, 3H), 6.98 (t, $J$ $=7.5 \mathrm{~Hz}, 1 \mathrm{H}), 6.84(\mathrm{~d}, J=7.5 \mathrm{~Hz}, 1 \mathrm{H}), 6.71(\mathrm{~d}, J=7 . .5 \mathrm{~Hz}, 1 \mathrm{H}), 5.35(\mathrm{~d}, J=7.6 \mathrm{~Hz}, 1 \mathrm{H}), 4.95(\mathrm{~d}, J=$ $15.7 \mathrm{~Hz}, 1 \mathrm{H}), 4.79$ (d, $J=15.8 \mathrm{~Hz}, 1 \mathrm{H}) .{ }^{13} \mathrm{C}$ NMR $\left(126 \mathrm{MHz}, \mathrm{CDCl}_{3}\right) \delta 175.80,165.57,142.66,136.15$, 135.48, 128.86, 128.82, 128.68, 127.69, 127.28, 126.92, 125.28, 124.17, 123.18, 122.65, 121.50, 120.55, 112.06, 109.78, 109.40, 53.16, 44.11. HRMS ESI (m/z): Calcd for $\mathrm{C}_{24} \mathrm{H}_{19} \mathrm{~N}_{3} \mathrm{O}_{2},[\mathrm{M}+\mathrm{H}]^{+}, 382.1550$; found: 382.1536 .

\section{N-(1-benzyl-2-oxoindolin-3-yl)thiophene-2-carboxamide (1t)}<smiles>CCc1ccccc1C1C(=O)N(Cc2ccccc2)c2ccccc21</smiles>

This compound was prepared according to typical procedure 2.2. Petroleum ether $/$ ethyl acetate $=5: 1$ was used as the eluent for column chromatography. White solid (609 mg, 70\%); m.p. 189.5-190.1 ${ }^{\circ} \mathrm{C}$; ${ }^{1} \mathbf{H}$ NMR (400 MHz, DMSO-d $\left.)\right) \delta 9.49$ (d, $\left.J=8.0 \mathrm{~Hz}, 1 \mathrm{H}\right), 7.86$ (dd, $\left.J=4.0,1.2 \mathrm{~Hz}, 1 \mathrm{H}\right), 7.82(\mathrm{dd}, J=$ 5.2, 1.2 Hz, 1H), 7.48-7.41 (m, 2H), 7.38-7.31 (m, 2H), 7.30-7.17 (m, 4H), 7.04-6.97 (m, 1H), 6.82 (d, $J$ $=7.6 \mathrm{~Hz}, 1 \mathrm{H}), 5.42(\mathrm{~d}, J=7.6 \mathrm{~Hz}, 1 \mathrm{H}), 4.93$ (dd, $J=23.2,16.0 \mathrm{~Hz}, 2 \mathrm{H}) .{ }^{13} \mathrm{C}$ NMR $(101 \mathrm{MHz}$, DMSO$\left.d_{6}\right) \delta 174.36,161.53,143.49,139.22,136.71,132.01,129.41,128.99,128.93,128.57,127.74,127.57$, 123.86, 122.73, 109.51, 52.91, 43.37. HRMS ESI (m/z): Calcd for $\mathrm{C}_{20} \mathrm{H}_{16} \mathrm{~N}_{2} \mathrm{O}_{2} \mathrm{~S},[\mathrm{M}+\mathrm{H}]^{+}, 349.1005$; found: 349.0990 .

\section{N-(1-benzyl-2-oxoindolin-3-yl)furan-2-carboxamide (1u)}<smiles>O=C(NC1C(=O)N(Cc2ccccc2)c2ccccc21)c1ccco1</smiles> 
This compound was prepared according to typical procedure 2.2. Petroleum ether / ethyl acetate $=5: 1$ was used as the eluent for column chromatography. White solid (581 mg, 70\%); m.p. 187.6-189.4 ${ }^{\circ} \mathrm{C}$; ${ }^{1} \mathbf{H}$ NMR (400 MHz, DMSO- $d_{6}$ ) $\delta 9.38$ (d, $\left.J=8.0 \mathrm{~Hz}, 1 \mathrm{H}\right), 7.90$ (dd, $J=1.6,0.8 \mathrm{~Hz}, 1 \mathrm{H}$ ), 7.46-7.41 ( m, 2H), 7.36-7.30 (m, 2H), 7.29-7.16 (m, 4H), 7.01- 6.96 (m, 1H), 6.78 (d, $J=7.6 \mathrm{~Hz}, 1 \mathrm{H}), 6.66$ (dd, $J=$ 3.6, 1.6 Hz, 1H), 5.39 (d, $J=8.0 \mathrm{~Hz}, 1 \mathrm{H}), 4.92$ (dd, $J=41.2,16.0 \mathrm{~Hz}, 2 \mathrm{H}) .{ }^{13} \mathrm{C}$ NMR $(101 \mathrm{MHz}$, DMSO$\left.d_{6}\right) \delta 174.30,158.07,147.61,146.00,143.49,136.67,128.95,128.88,127.70,127.56,123.81,122.67$, 114.75, 112.49, 109.49, 52.45, 43.36. HRMS ESI (m/z): Calcd for $\mathrm{C}_{20} \mathrm{H}_{16} \mathrm{~N}_{2} \mathrm{O}_{3},[\mathrm{M}+\mathrm{H}]^{+}, 331.1088$; found: 331.1087 .

N-(1-benzyl-2-oxoindolin-3-yl)cyclopropanecarboxamide (1v)<smiles>O=C(NC1C(=O)N(Cc2ccccc2)c2ccccc21)C1CC1</smiles>

This compound was prepared according to typical procedure 2.2. Petroleum ether $/$ ethyl acetate $=4: 1$ was used as the eluent for column chromatography. White solid (482 mg, 63\%); m.p. 190.4-191.7 ${ }^{\circ} \mathrm{C}$; ${ }^{1} \mathbf{H}$ NMR (400 MHz, $\mathrm{CDCl}_{3}$ ) $\delta$ 7.38-7.29 (m, 5H), 7.21-715 (m, 1H), 7.05-6.99 (m, 1H), 6.71 (d, $J=8.0$ Hz, 1H), 6.49 (d, $J=7.2 \mathrm{~Hz}, 1 \mathrm{H}$ ), 5.43 (d, $J=7.6 \mathrm{~Hz}, 1 \mathrm{H}$ ), 4.91 (dd, $J=55.2,16.0 \mathrm{~Hz}, 2 \mathrm{H}$ ), 1.54-1.46 (m, 1H), 1.09-1.00 (m, 2H), 0.86-0.77 (m, 2H). ${ }^{13}$ C NMR (101 MHz, $\left.\mathrm{CDCl}_{3}\right) \delta 174.56,174.20,142.83$, 135.46, 129.08, 128.83, 127.72, 127.34, 126.74, 124.68, 123.05, 109.32, 52.89, 44.14, 14.62, 7.97, 7.90. HRMS ESI (m/z): Calcd for $\mathrm{C}_{19} \mathrm{H}_{18} \mathrm{~N}_{2} \mathrm{O}_{2}$, [M-H]', 305.1296; found: 305.1297.

\section{N-(1-benzyl-2-oxoindolin-3-yl)cyclopentanecarboxamide (1w)}<smiles>O=C(NC1C(=O)N(Cc2ccccc2)c2ccccc21)C1CCCC1</smiles>

This compound was prepared according to typical procedure 2.2. Petroleum ether $/$ ethyl acetate $=4: 1$ was used as the eluent for column chromatography. White solid (492 mg, 59\%); m.p. 187.5-189.7 ${ }^{\circ} \mathrm{C}$; ${ }^{1} \mathbf{H}$ NMR (400 MHz, DMSO-d $\left.d_{6}\right) \delta .71$ (d, $\left.J=8.0 \mathrm{~Hz}, 1 \mathrm{H}\right), 7.39$ (d, $\left.J=7.2 \mathrm{~Hz}, 2 \mathrm{H}\right), 7.34-7.29(\mathrm{~m}, 2 \mathrm{H})$, 7.28-7.23(m, 1H), 7.20-7.13 (m, 2H), 7.01-6.95 (m, 1H), 6.77 (d, $J=7.6 \mathrm{~Hz}, 1 \mathrm{H}), 5.23$ (d, $J=8.0 \mathrm{~Hz}$, 1H), 4.89 (dd, $J=25.2,16.0$ Hz, 2H), 2.71-2.61 (m, 1H), 1.84 -1.74 (m, 2H), 1.72-1.59 (m, 4H), 1.551.46 (m, 2H). ${ }^{13}$ C NMR (101 MHz, DMSO-d $\left.{ }_{6}\right) \delta 175.97,174.59,143.34,136.74,128.96,128.73,128.04$, 127.69, 123.60, 122.66, 109.40, 52.45, 44.23, 43.25, 30.39, 26.18, 26.15. HRMS ESI (m/z): Calcd for $\mathrm{C}_{21} \mathrm{H}_{22} \mathrm{~N}_{2} \mathrm{O}_{2},[\mathrm{M}+\mathrm{H}]^{+}$, 335.1754; found: 335.1741.

\section{N-(1-benzyl-2-oxoindolin-3-yl)tetrahydro-2H-pyran-4-carboxamide (1x)}


<smiles>[Y4]c1ccccc1C1CC(C(=O)C2CCOCC2)N(Cc2ccccc2)C1=O</smiles>

This compound was prepared according to typical procedure 2.2. Petroleum ether / ethyl acetate $=4: 1$ was used as the eluent for column chromatography. White solid (116 mg, 33\%); m.p. 174.6-176.9 ${ }^{\circ} \mathrm{C}$; ${ }^{1} \mathbf{H}$ NMR (500 MHz, $\mathrm{CDCl}_{3}$ ) $\delta$ 7.36-7.27 (m, 6H), 7.23-7.16 (m, 1H), 7.06-6.99 (m, 1H), 6.73 (d, $J=7.5$ $\mathrm{Hz}, 1 \mathrm{H}), 6.29$ (d, $J=5.0 \mathrm{~Hz}, 1 \mathrm{H}), 5.41$ (d, $J=6.5 \mathrm{~Hz}, 1 \mathrm{H}), 4.90$ (dd, $J=65.5,16.0 \mathrm{~Hz}, 2 \mathrm{H}), 4.07-3.96$ (m, 2H), 3.46-3.36 (m, 2H), 2.54-2.43(m, 1H), 1.92-1.79 (m, 4H). ${ }^{13} \mathbf{C}$ NMR (126 MHz, CDCl $) \delta 174.80$, 174.35, 142.88, 135.38, 129.18, 128.85, 127.77, 127.34, 126.53, 124.43, 123.12, 109.40, 67.16, 67.14, 52.60, 44.15, 41.77, 29.24, 29.10. HRMS ESI (m/z): Calcd for $\mathrm{C}_{21} \mathrm{H}_{22} \mathrm{~N}_{2} \mathrm{O}_{3}$, [M+H] $]^{+}, 351.1703$; found: 351.1710 .

ethyl (1-benzyl-2-oxoindolin-3-yl)carbamate (1y)<smiles>CCOC(=O)NC1C(=O)N(Cc2ccccc2)c2ccccc21</smiles>

This compound was prepared according to typical procedure 2.1. Petroleum ether / ethyl acetate $=5: 1$ was used as the eluent for column chromatography. White solid (1.02 g, 83\%); m.p.152.5-153.9 ${ }^{\circ} \mathrm{C} .{ }^{1} \mathbf{H}$ NMR (400 MHz, $\left.\mathrm{CDCl}_{3}\right) \delta$ 7.39-7.29 (m, 5H), 7.28-7.22 (m, 1H), 7.18 (t, $\left.J=7.6 \mathrm{~Hz}, 1 \mathrm{H}\right)$, 7.05-7.00 (m, 1H), 6.71 (d, $J=8.0 \mathrm{~Hz}, 1 \mathrm{H}), 5.47$ (d, $J=6.8 \mathrm{~Hz}, 1 \mathrm{H}), 5.16$ (d, $J=4.8 \mathrm{~Hz}, 1 \mathrm{H}), 4.91$ (dd, $J=40.0,16.0$ Hz, 2H), 4.17 (d, $J=6.8 \mathrm{~Hz}, 2 \mathrm{H}), 1.25$ (s, 3H). ${ }^{13} \mathbf{C}$ NMR (126 MHz, $\left.\mathrm{CDCl}_{3}\right) \delta 174.46,156.47,142.84$, 135.46, 129.20, 128.80, 127.70, 127.34, 126.43, 124.41, 122.98, 109.35, 61.65, 53.87, 44.10, 14.49. HRMS ESI (m/z): Calcd for $\mathrm{C}_{18} \mathrm{H}_{18} \mathrm{~N}_{2} \mathrm{O}_{3}$, [M+H] $]^{+}, 311.1390$; found: 311.1395.

\section{Typical procedures and characterization data for compounds $3 a-3 y$}

\subsection{General procedure for the synthesis of compounds 3a-3y}<smiles>[R][R]1ccc2c(c1)[C@@H](NC([R])=O)C(=O)N2[R]</smiles>

A solution of 3-benzamido-2-oxindole 1a-1y (0.1 mmol), ethylene sulfonyl fluoride $2 \mathbf{a}$ (13.2 mg, 0.12 mmol) and catalyst $4 \mathbf{d}(12.6 \mathrm{mg}, 0.02 \mathrm{mmol})$ in $\mathrm{CH}_{2} \mathrm{Cl}_{2}(1.0 \mathrm{~mL})$ was stirred at rt for $12 \mathrm{~h}$. After the solvent was evaporated under vacuum, the residue was purified by flash column chromatography over silica gel (petroleum ether / ethyl acetate $=8: 1$ ) to afford the product $\mathbf{3 a - 3 y}$. 
3.2 General procedure for the synthesis of racemic products 3a-3y

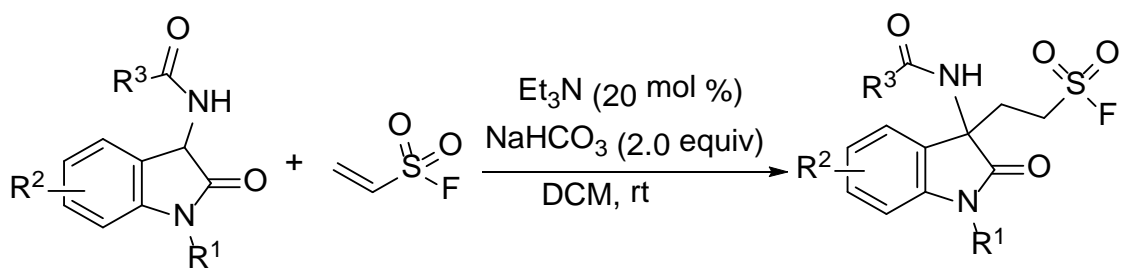

A solution of 3-benzamido-2-oxindole 1a-1y (0.1 mmol), ethylene sulfonyl fluoride $\mathbf{2 a}$ (13.2 $\mathrm{mg}, 0.12$ mmol), $\mathrm{NaHCO}_{3}(16.8 \mathrm{mg}, 0.2 \mathrm{mmol})$ and $\mathrm{Et}_{3} \mathrm{~N}(3 \mathrm{mg}, 0.02 \mathrm{mmol})$ in $\mathrm{CH}_{2} \mathrm{Cl}_{2}(1.0 \mathrm{~mL})$ was stirred at $\mathrm{rt}$ for $12 \mathrm{~h}$. After the solvent was evaporated under vacuum, the residue was purified by flash column chromatography over silica gel (petroleum ether / ethyl acetate $=8: 1$ ) to afford the racemic products 3a-3y.

\subsection{Characterizations of products $3 a-3 y$}

(S)-2-(3-benzamido-1-benzyl-2-oxoindolin-3-yl)ethane-1-sulfonyl fluoride (3a)<smiles>O=C(N[C@]1(CCS(=O)(=O)F)C(=O)Nc2ccccc21)c1ccccc1</smiles>

3a

Petroleum ether / ethyl acetate $=8: 1$ was used as the eluent for column chromatography. White solid (44.5 mg, 98\%), m.p. $172.1-173.5^{\circ} \mathrm{C} .{ }^{1} \mathbf{H}$ NMR $\left(500 \mathrm{MHz}, \mathrm{CDCl}_{3}\right)$ $\delta$ 7.79-7.72 (m, 2H), 7.51-7.46 (m, 1H), 7.43-7.34 (m, 7H), 7.33-7.26 (m, 2H), 7.25-7.20 (m, 1H), 7.05 (td, $J=7.5,0.5 \mathrm{~Hz}, 1 \mathrm{H}), 6.79$ (d, $J$ $=8.0 \mathrm{~Hz}, 1 \mathrm{H}), 4.99(\mathrm{dd}, J=31.5,16.0 \mathrm{~Hz}, 2 \mathrm{H}), 3.72-3.63(\mathrm{~m}, 1 \mathrm{H}), 3.49-3.40(\mathrm{~m}, 1 \mathrm{H}), 2.82$ (ddd, $J=$ 14.0, 10.0, $5.0 \mathrm{~Hz}, 1 \mathrm{H}), 2.49$ (ddd, $J=14.5,10.0,5.0 \mathrm{~Hz}, 1 \mathrm{H}) .{ }^{13} \mathrm{C} \mathbf{N M R}\left(126 \mathrm{MHz}, \mathrm{CDCl}_{3}\right) \delta 175.02$, 166.71, 142.19, 135.28, 132.35, 132.26, 129.81, 128.99, 128.63, 128.31, 127.89, 127.34, 127.29, 123.60 , 123.01, 109.99, 59.92, 44.95 (d, $\left.J_{\mathrm{C}-\mathrm{F}}=18.1 \mathrm{~Hz}\right), 44.39,30.40 .{ }^{19} \mathbf{F}$ NMR $\left(471 \mathrm{MHz}, \mathrm{CDCl}_{3}\right) \delta 53.31$. HRMS ESI (m/z): Calcd for $\mathrm{C}_{24} \mathrm{H}_{21} \mathrm{FN}_{2} \mathrm{O}_{4} \mathrm{~S}$, [M+Na] $]^{+}$, 475.3274; found: 475.3276. [ $\left.\boldsymbol{\alpha}\right]_{\mathrm{D}}^{20}=+25.8$ (c 1.0, $\mathrm{CH}_{2} \mathrm{Cl}_{2}$ ). Chiral HPLC analysis (Chiral pak AS-H, $i-\mathrm{PrOH} / n$-hexane $=20: 80$, flow rate $=0.5 \mathrm{~mL} / \mathrm{min}$, wave length $=254 \mathrm{~nm}), \mathrm{t}_{\mathrm{R}}($ minor enantiomer $)=16.959 \mathrm{~min}, \mathrm{t}_{\mathrm{R}}($ major enantiomer $)=32.849 \mathrm{~min}, 99 \%$ ee. 


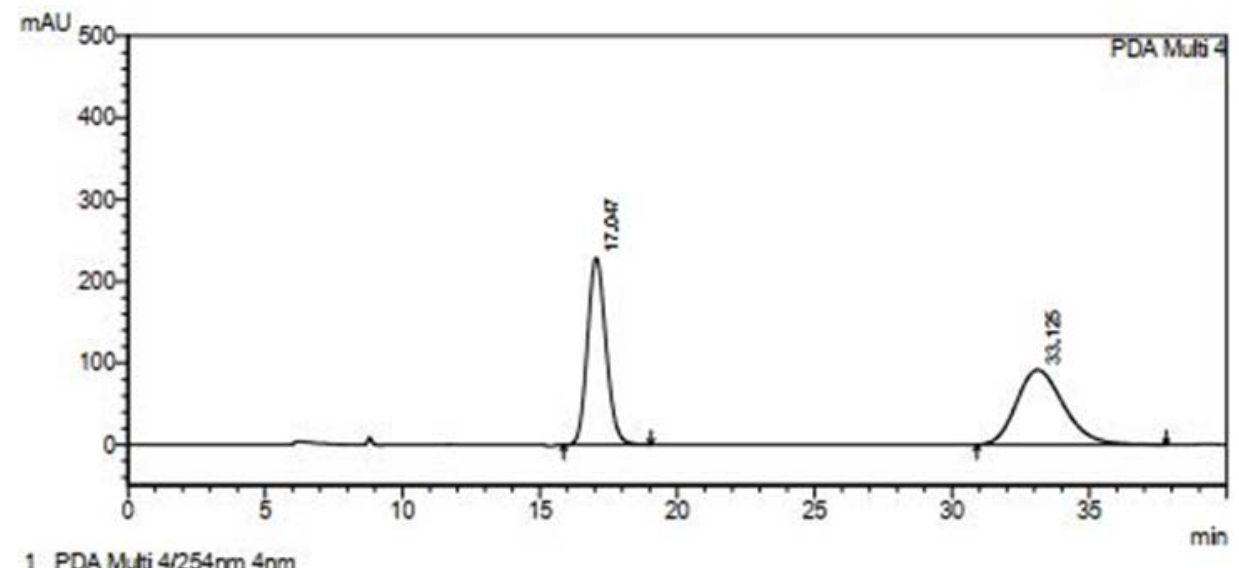

1 PDA Muti $4 / 254 \mathrm{~mm} 4 \mathrm{~nm}$
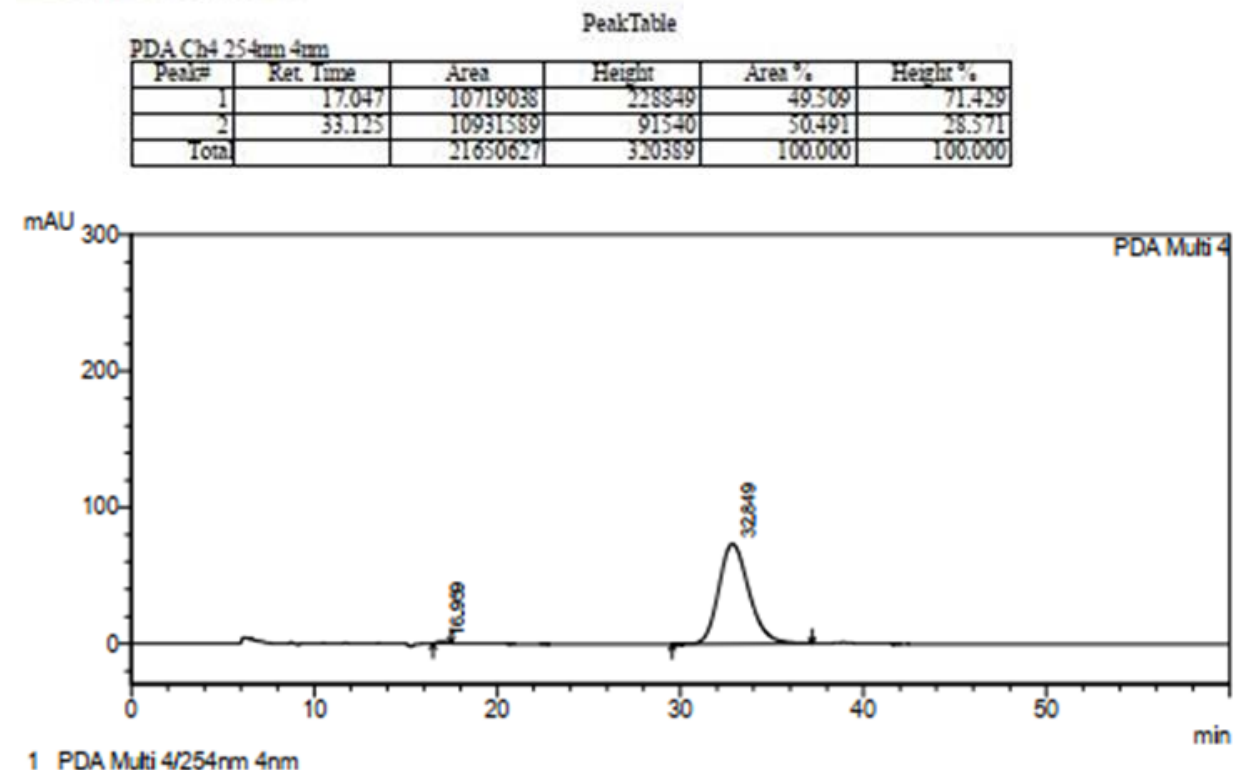

1 PDA Muti $4 / 254 \mathrm{~mm} 4 \mathrm{~nm}$

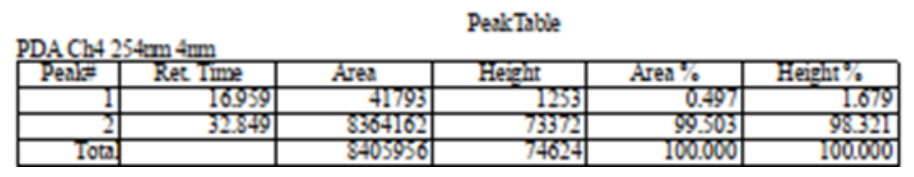

(S)-2-(3-benzamido-2-oxo-1-phenylindolin-3-yl)ethane-1-sulfonyl fluoride (3b)<smiles>O=C(N[C@]1(CCS(=O)(=O)F)C(=O)Nc2ccccc21)c1ccccc1</smiles>

$\mathrm{Ph}$

3b

Petroleum ether / ethyl acetate $=8: 1$ was used as the eluent for column chromatography. White solid (38.5 mg, 88\%), m. p. 183.6-184.3 ${ }^{\circ} \mathrm{C} .{ }^{1} \mathbf{H}$ NMR (500 MHz, $\left.\mathrm{CDCl}_{3}\right) \delta$ 7.79-7.74 (m, 2H), 7.55-7.49 (m, 4H), 7.49-7.42 (m, 3H), 7.37 (t, $J=7.5 \mathrm{~Hz}, 2 \mathrm{H}), 7.32-7.25$ (m, 2H), 7.12-7.07 (m, 1H), 6.83 (d, $J=8.0$ Hz, 1H), 3.86-3.77 ( m, 1H), 3.57-3.48 (m, 1H), 2.87-2.78 (m, 1H), 2.60-2.51 (m, 1H). ${ }^{13}$ C NMR (126 $\left.\mathrm{MHz}, \mathrm{CDCl}_{3}\right) \delta 174.68,166.91,143.60,134.14,132.33,132.30,129.84,128.69,128.66,128.04,127.41$, 
126.90, 123.93, 123.04, 110.13, 59.92, 44.99 (d, $\left.J_{\text {C-F }}=18.1 \mathrm{~Hz}\right), 30.21 .{ }^{19} \mathbf{F}$ NMR $\left(471 \mathrm{MHz}, \mathrm{CDCl}_{3}\right) \delta$ 53.38. HRMS ESI (m/z): Calcd for $\mathrm{C}_{23} \mathrm{H}_{19} \mathrm{FN}_{2} \mathrm{O}_{4} \mathrm{~S}$, $[\mathrm{M}+\mathrm{Na}]^{+}$, 461.0942; found: 461.0946. [ $\left.\boldsymbol{\alpha}\right]_{\mathrm{D}}{ }^{20}=+$ 26.2 (с 1.0, $\mathrm{CH}_{2} \mathrm{Cl}_{2}$ ). Chiral HPLC analysis (Chiral pak AS-H, $i-\mathrm{PrOH} / n$-hexane $=20: 80$, flow rate $=$ $0.5 \mathrm{~mL} / \mathrm{min}$, wave length $=254 \mathrm{~nm}), \mathrm{t}_{\mathrm{R}}($ minor enantiomer $)=13.335 \mathrm{~min}, \mathrm{t}_{\mathrm{R}}($ major enantiomer $)=30.536$ $\min , 99 \%$ ee.

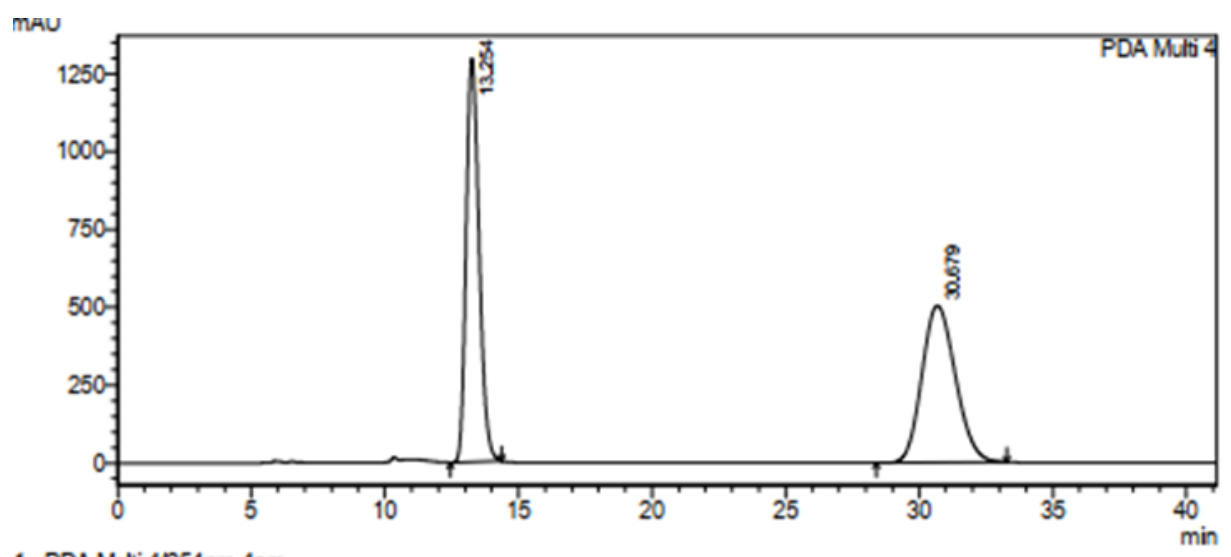

1 PDA Muti $4 / 254 \mathrm{~mm} 4 \mathrm{~nm}$
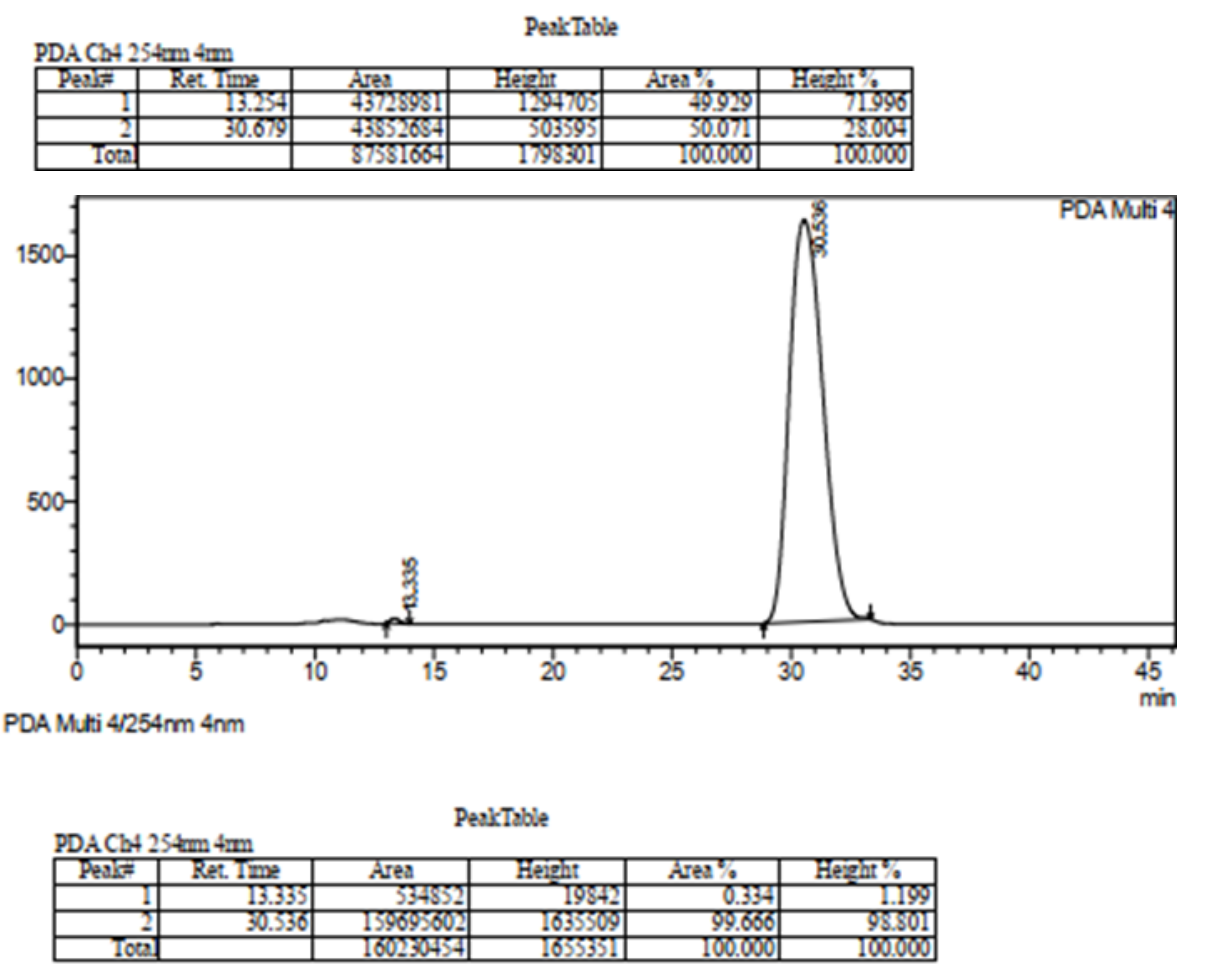

(S)-2-(3-benzamido-1-methyl-2-oxoindolin-3-yl)ethane-1-sulfonyl fluoride (3c)<smiles>CN1C(=O)C(CCS(=O)(=O)F)(NC(=O)c2ccccc2)c2ccccc21</smiles> 
Petroleum ether / ethyl acetate $=8: 1$ was used as the eluent for column chromatography. White solid (32 mg, 85\%), m. p. 187.3-188.9 ㄷ. ${ }^{1} \mathbf{H}$ NMR (400 MHz, $\left.\mathrm{CDCl}_{3}\right) \delta 7.77$ (d, $\left.J=7.6 \mathrm{~Hz}, 2 \mathrm{H}\right), 7.55-7.35$ (m, 5H), 7.16-7.09 (m, 1H), 7.04 (s, 1H), 6.94 (d, $J=8.0$ Hz, 1H), 3.76 (ddd, $J=15.6,10.8,6.0$ Hz, 1H), 3.54-3.44 (m, 1H), 3.32 (s, 3H), 2.88 (ddd, $J=14.4,9.6,5.2 \mathrm{~Hz}, 1 \mathrm{H}), 2.49-2.38$ (m, 1H). ${ }^{13} \mathrm{C}$ NMR (101 $\left.\mathrm{MHz}_{\mathrm{CDCl}}\right) \delta 174.86,166.87,143.19,132.43,132.31,130.01,128.67,128.34,127.34,123.61,123.20$, 108.93, 59.91, 45.04 (d, $\left.J_{\mathrm{C}-\mathrm{F}}=18.0 \mathrm{~Hz}\right), 29.96,26.80 .{ }^{19} \mathbf{F}$ NMR $\left(376 \mathrm{MHz}, \mathrm{CDCl}_{3}\right) \delta 53.16$. HRMS ESI $\left(\mathrm{m} / \mathrm{z} \text { ): Calcd for } \mathrm{C}_{18} \mathrm{H}_{17} \mathrm{~N}_{2} \mathrm{O}_{4} \mathrm{FS} \text {, [M+Na] }\right]^{+}$, 399.0785; found: 399.0787. [ $\left.\boldsymbol{\alpha}\right]_{\mathrm{D}}{ }^{20}=+32.5$ (c 1.0, $\mathrm{CH}_{2} \mathrm{Cl}_{2}$ ). Chiral HPLC analysis (Chiral pak AS-H, $i$-PrOH $/ n$-hexane $=20: 80$, flow rate $=0.5 \mathrm{~mL} / \mathrm{min}$, wave length $=254 \mathrm{~nm}), \mathrm{t}_{\mathrm{R}}($ minor enantiomer $)=13.405 \mathrm{~min}, \mathrm{t}_{\mathrm{R}}($ major enantiomer $)=30.599 \mathrm{~min}, 99 \%$ ee.

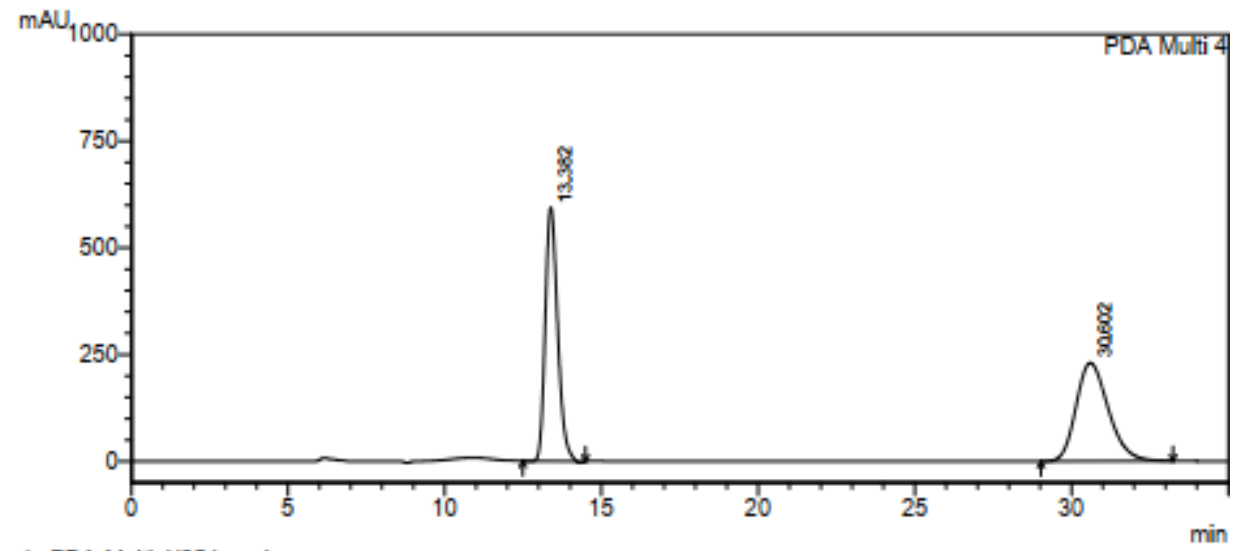

1 PDA Multi 4/254nm 4nm

PeakTable

\begin{tabular}{|c|c|c|c|c|c|}
\hline \multicolumn{6}{|c|}{ PDA Cb4 254am $4 \mathrm{~nm}$} \\
\hline Pealk & Rec. Time & Areas & Height & Area \% & Height \% \\
\hline 1 & 13.382 & 10271949 & 594225 & 49.685 & 72.098 \\
\hline 2 & 30.002 & 16478272 & 229971 & 50.315 & 27902 \\
\hline Toca & & 32750221 & 824196 & 100.000 & 100.000 \\
\hline
\end{tabular}
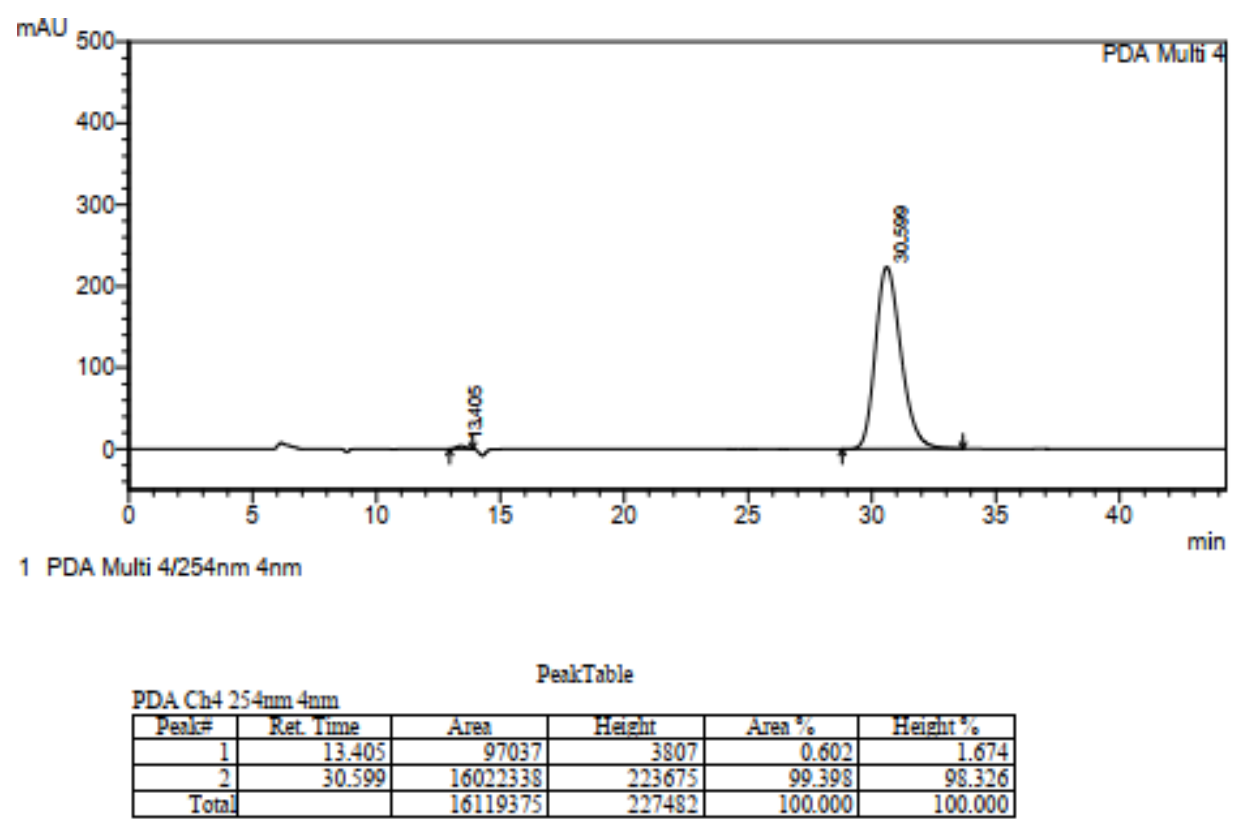

(S)-2-(3-benzamido-1-benzyl-5-fluoro-2-oxoindolin-3-yl)ethane-1-sulfonyl fluoride (3d) 
<smiles>O=C(N[C@]1(CCS(=O)(=O)F)C(=O)Nc2ccc(F)cc21)c1ccccc1</smiles>

Petroleum ether / ethyl acetate $=8: 1$ was used as the eluent for column chromatography. White solid (45.6 mg, 97\%), m. p. 227.5-228.5 ${ }^{\circ} \mathrm{C} .{ }^{1} \mathbf{H}$ NMR (400 MHz, $\left.\mathrm{CDCl}_{3}\right) \delta$ 7.79-7.71 (m, 2H), 7.51 (t, $J=7.6$ Hz, 1H), 7.43-7.27 (m, 8H), 7.14 (dd, $J=7.6,2.8 \mathrm{~Hz}, 1 \mathrm{H}$ ), 6.92 (td, $J=8.8,2.8 \mathrm{~Hz}, 1 \mathrm{H}$ ), 6.70 (dd, $J=$ 8.4, $4.0 \mathrm{~Hz}, 1 \mathrm{H}), 5.02-4.93(\mathrm{~m}, 2 \mathrm{H}), 3.77-3.67(\mathrm{~m}, 1 \mathrm{H}), 3.53-3.42(\mathrm{~m}, 1 \mathrm{H}), 2.87-2.76(\mathrm{~m}, 1 \mathrm{H}), 2.52-2.41$ (m, 1H). ${ }^{13}$ C NMR (101 MHz, CDCl $) \delta 174.91,166.86,159.62\left(\mathrm{~d}, J_{\mathrm{C}-\mathrm{F}}=243.2 \mathrm{~Hz}\right), 138.06$ (d, $J_{\mathrm{C}-\mathrm{F}}=$ $2.1 \mathrm{~Hz}$ ), 134.94, 132.46, 132.09, 130.05 (d, $J_{\mathrm{C}-\mathrm{F}}=7.8 \mathrm{~Hz}$ ), 129.10, 128.73, 128.05, 127.38, 127.28, $116.16\left(\mathrm{~d}, J_{\mathrm{C}-\mathrm{F}}=23.5 \mathrm{~Hz}\right), 111.35\left(\mathrm{~d}, J_{\mathrm{C}-\mathrm{F}}=25.3 \mathrm{~Hz}\right), 110.81\left(\mathrm{~d}, J_{\mathrm{C}-\mathrm{F}}=8.0 \mathrm{~Hz}\right), 60.12,44.82\left(\mathrm{~d}, J_{\mathrm{C}-\mathrm{F}}=\right.$ 18.3 Hz), 44.57, 30.27. ${ }^{19} \mathbf{F}$ NMR (376 MHz, $\left.\mathrm{CDCl}_{3}\right) \delta 53.40,-118.35$. HRMS ESI (m/z): Calcd for $\mathrm{C}_{24} \mathrm{H}_{20} \mathrm{~N}_{2} \mathrm{O}_{4} \mathrm{~F}_{2} \mathrm{~S}$, $[\mathrm{M}+\mathrm{Na}]^{+}$, 493.1004; found: 493.1014. $[\alpha]_{\mathrm{D}}{ }^{20}=+7.9$ (c 1.0, $\mathrm{CH}_{2} \mathrm{Cl}_{2}$ ). Chiral HPLC analysis (Chiral pak AS-H, $i-\mathrm{PrOH} / n$-hexane $=20: 80$, flow rate $=0.5 \mathrm{~mL} / \mathrm{min}$, wave length $=254 \mathrm{~nm}$ ), $t_{R}($ minor enantiomer $)=14.508$ min, $t_{R}($ major enantiomer $)=27.752 \mathrm{~min}, 98 \%$ ee.

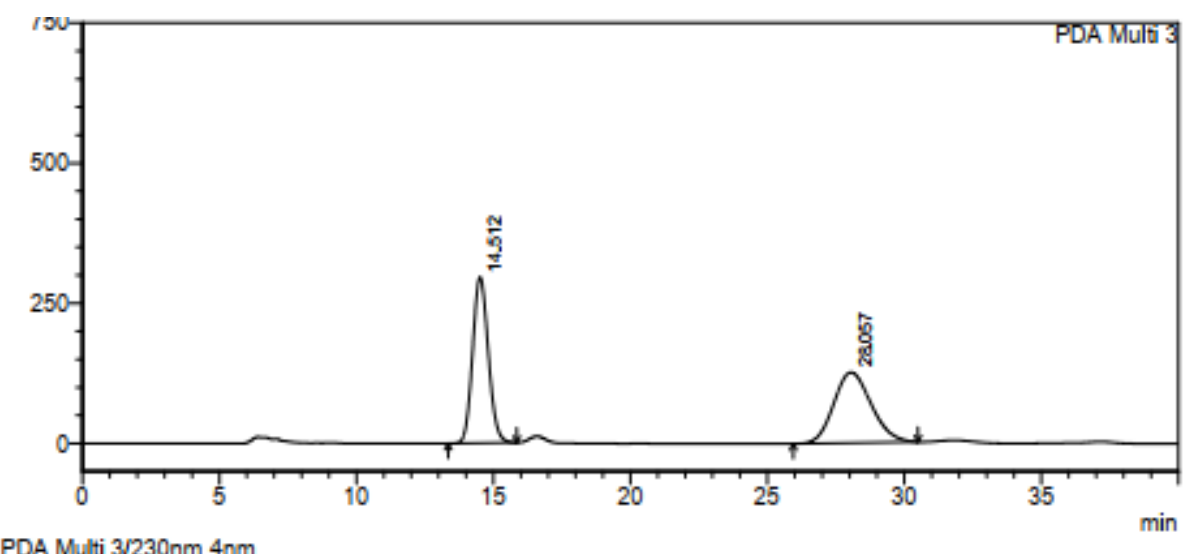

PDA Multi $3 / 230 \mathrm{~nm} 4 \mathrm{~nm}$

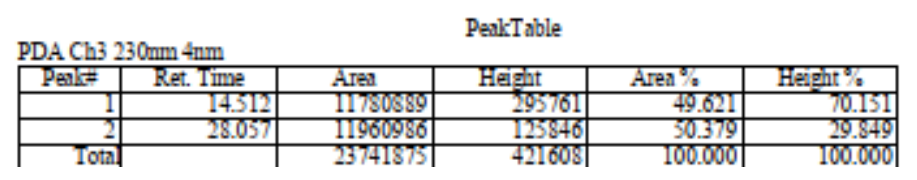




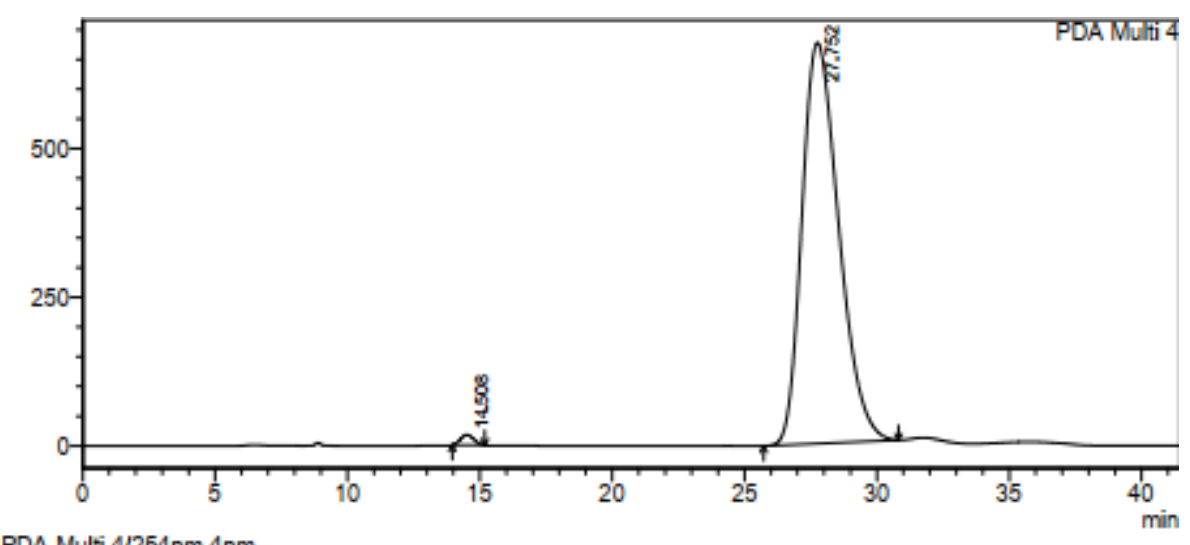

PDA Multi 4/254nm 4nm

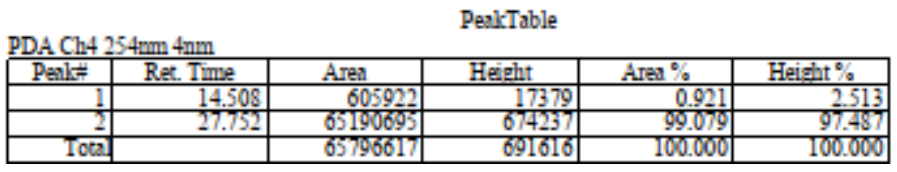

(S)-2-(3-benzamido-1-benzyl-5-chloro-2-oxoindolin-3-yl)ethane-1-sulfonyl fluoride (3e)<smiles>O=C(N[C@]1(CCS(=O)(=O)F)C(=O)Nc2ccc(Cl)cc21)c1ccccc1</smiles>

$3 e$

Petroleum ether / ethyl acetate $=8: 1$ was used as the eluent for column chromatography. White solid (48.5 mg, 99\%), m. p. $246.7-247.5{ }^{\circ} \mathrm{C} .{ }^{1} \mathbf{H}$ NMR (400 MHz, $\left.\mathrm{CDCl}_{3}\right) \delta 7.75$ (d, $\left.J=7.6 \mathrm{~Hz}, 2 \mathrm{H}\right), 7.54-$ 7.48 (m, 1H), 7.44-7.28 (m, 9H), 7.20 (d, $J=8.0 \mathrm{~Hz}, 1 \mathrm{H}), 6.70$ (d, $J=8.4 \mathrm{~Hz}, 1 \mathrm{H}), 4.97$ (s, 2H), 3.81$3.69(\mathrm{~m}, 1 \mathrm{H}), 3.55$-3.44 (m, 1H), 2.85-2.73 (m, 1H), 2.50-2.39 (m, 1H). ${ }^{13} \mathbf{C}$ NMR (101 MHz, $\left.\mathrm{CDCl}_{3}\right) \delta$ 174.69, 166.82, 140.71, 134.83, 132.50, 132.01, 130.15, 129.78, 129.11, 128.75, 128.09, 127.41, 127.27, 123.41, 111.07, 59.83, 44.75 (d, $\left.J_{\mathrm{C}-\mathrm{F}}=18.2 \mathrm{~Hz}\right), 44.55,30.27 .{ }^{19} \mathbf{F} \mathbf{~ N M R}\left(376 \mathrm{MHz}, \mathrm{CDCl}_{3}\right) \delta 53.40$. HRMS ESI (m/z): Calcd for $\mathrm{C}_{24} \mathrm{H}_{20} \mathrm{~N}_{2} \mathrm{O}_{4} \mathrm{FSCl},[\mathrm{M}+\mathrm{H}]^{+}$, 487.0889; found: 487.0868. [ $[\boldsymbol{\alpha}]_{\mathrm{D}}^{20}=-18.6$ (c 1.0, $\mathrm{CH}_{2} \mathrm{Cl}_{2}$ ). Chiral HPLC analysis (Chiral pak AS-H, $i-\mathrm{PrOH} / n$-hexane $=20: 80$, flow rate $=0.5$ $\mathrm{mL} / \mathrm{min}$, wave length $=230 \mathrm{~nm}), \mathrm{t}_{\mathrm{R}}($ minor enantiomer $)=13.165 \mathrm{~min}, \mathrm{t}_{\mathrm{R}}($ major enantiomer $)=25.218$ min, $98 \%$ ee. 


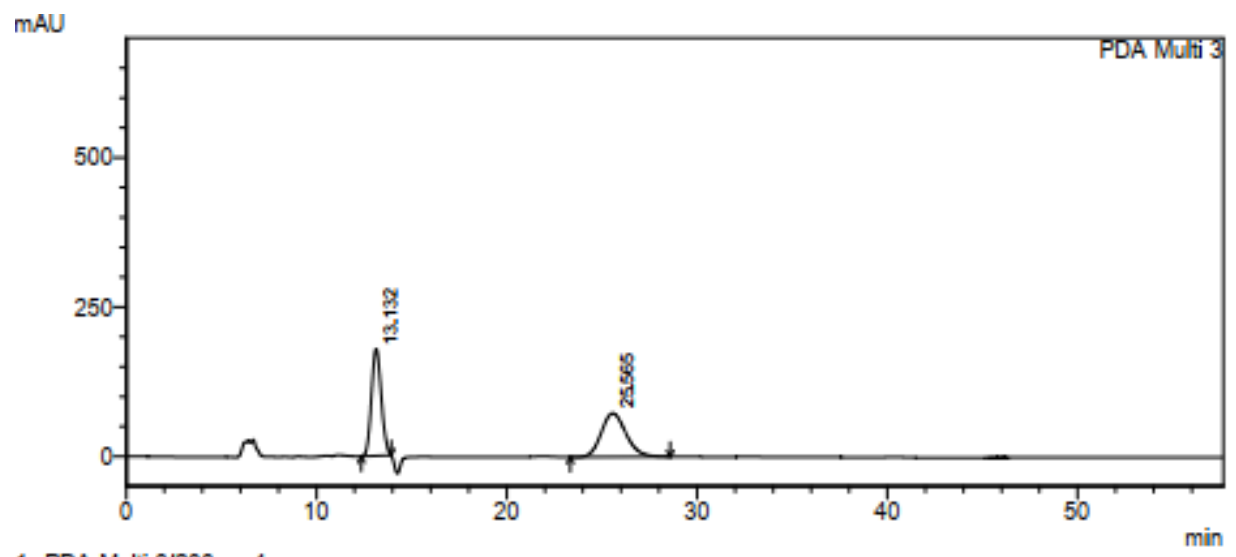

1 PDA Multi $3 / 230 \mathrm{~nm} 4 \mathrm{~nm}$

PeakTable
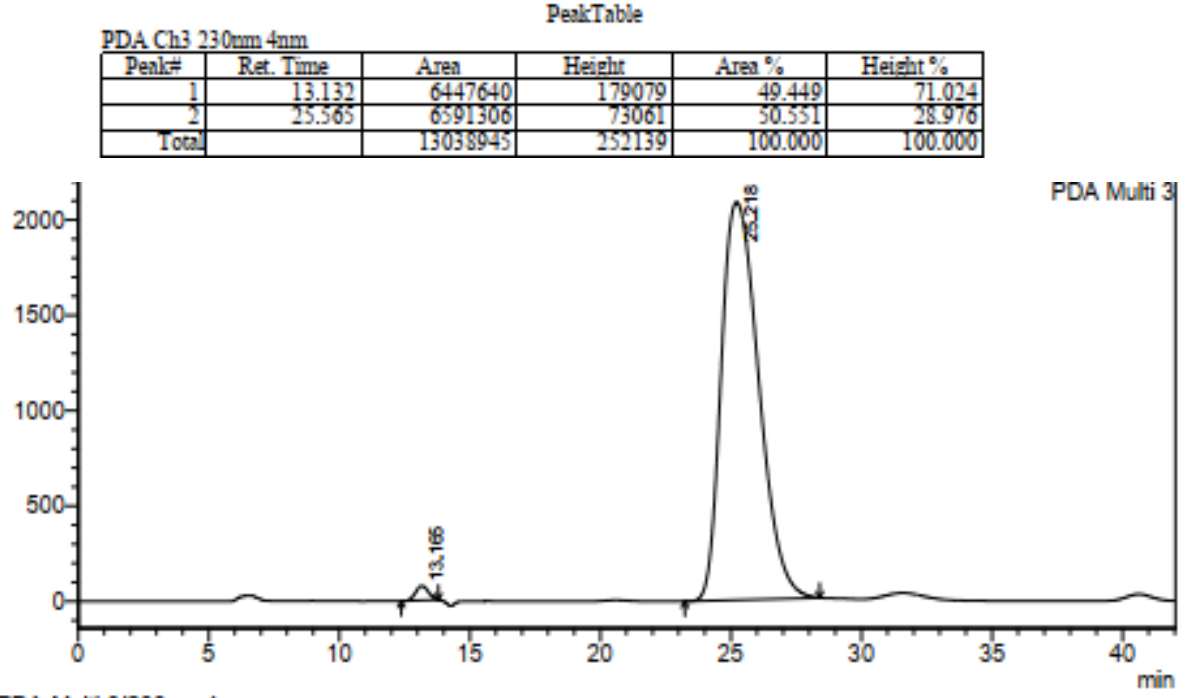

PDA Multi $3 / 230 \mathrm{~nm} 4 \mathrm{~nm}$

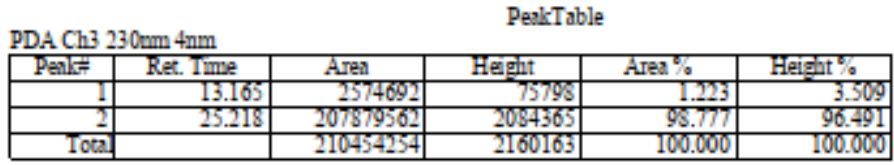

(S)-2-(3-benzamido-1-benzyl-5-bromo-2-oxoindolin-3-yl)ethane-1-sulfonyl fluoride (3f)<smiles>O=C(P)N[C@]1(CCS(=O)(=O)F)C(=O)N(Cc2ccccc2)c2ccc(Br)cc21</smiles>

Petroleum ether / ethyl acetate $=8: 1$ was used as the eluent for column chromatography. White solid (51.6 mg, 98\%), m. p. 201.8-203.4 ${ }^{\circ} \mathrm{C} .{ }^{1} \mathbf{H}$ NMR $\left(500 \mathrm{MHz}, \mathrm{CDCl}_{3}\right) \delta 7.79$ (d, $J=7.5 \mathrm{~Hz}, 2 \mathrm{H}$ ), 7.577.49 (m, 2H), 7.43 (t, $J=7.5 \mathrm{~Hz}, 2 \mathrm{H}), 7.41-7.33$ (m, 5H), 7.33-7.28 (m, 1H), 7.23 (s, 1H), 6.66 (d, $J=$ $8.5 \mathrm{~Hz}, 1 \mathrm{H}$ ), 4.99 (s, 2H), 3.87-3.78 (m, 1H), 3.56-3.47 (m, 1H), 2.83 (ddd, $J=14.5,9.0,5.5 \mathrm{~Hz}, 1 \mathrm{H}$ ), 2.42 (ddd, $J=15.0,9.5,6.0 \mathrm{~Hz}, 1 \mathrm{H}) .{ }^{13} \mathrm{C}$ NMR $\left(126 \mathrm{MHz}, \mathrm{CDCl}_{3}\right) \delta 174.43,166.74,141.19,134.79$, 132.67, 132.52, 132.04, 130.52, 129.09, 128.75, 128.06, 127.36, 127.25, 126.17, 116.31, 111.49, 59.60, $44.67\left(\mathrm{~d}, J_{\mathrm{C}-\mathrm{F}}=18.3 \mathrm{~Hz}\right), 44.50,30.24 .{ }^{19} \mathbf{F}$ NMR $\left(471 \mathrm{MHz}, \mathrm{CDCl}_{3}\right) \delta 53.33$. HRMS ESI $(\mathrm{m} / \mathrm{z})$ : Calcd 


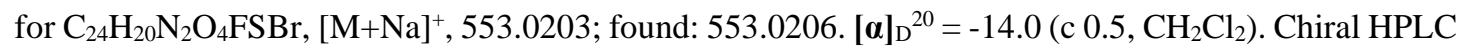
analysis (Chiral pak AS-H, $i$-PrOH$/ n$-hexane $=20: 80$, flow rate $=0.5 \mathrm{~mL} / \mathrm{min}$, wave length $=254 \mathrm{~nm}$ ), $t_{R}($ minor enantiomer $)=14.052$ min, $t_{R}$ (major enantiomer $)=28.872 \mathrm{~min}, 99 \%$ ee.

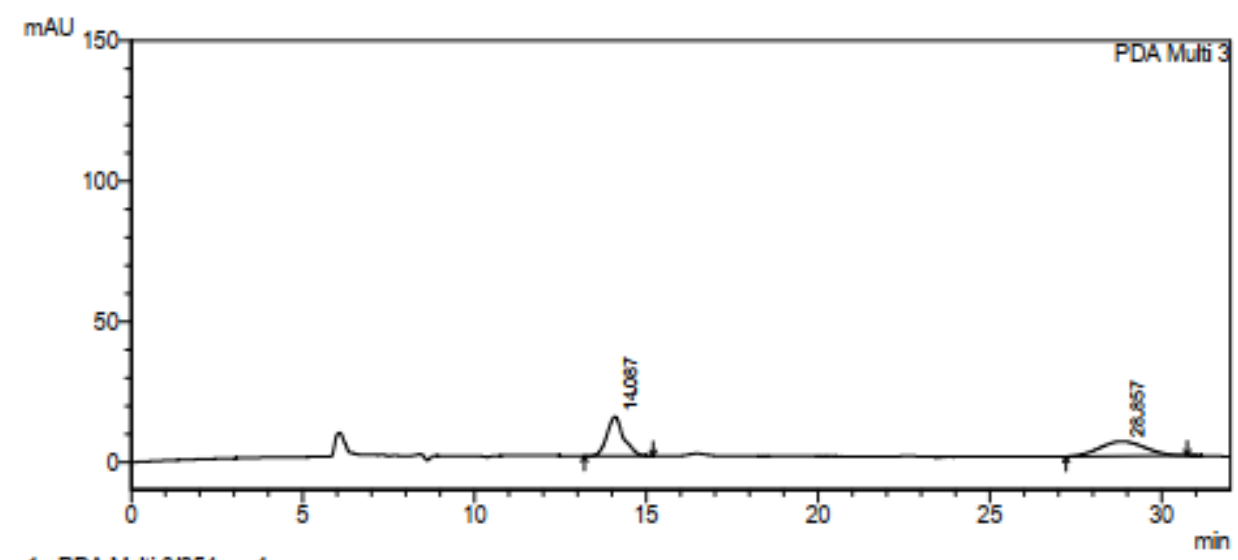

1 PDA Multi $3 / 254 \mathrm{~mm} 4 \mathrm{~nm}$

PeakTable

\begin{tabular}{|c|c|c|c|c|c|}
\hline Peali= & Ret. Tme & Area & Height & Area \% & Height \% \\
\hline & 14.087 & 475336 & 13976 & 49.975 & 72.768 \\
\hline & 28.859 & 475804 & 3230 & 50.025 & 27.232 \\
\hline Tota & & 951140 & 19206 & 100.000 & 100.000 \\
\hline
\end{tabular}

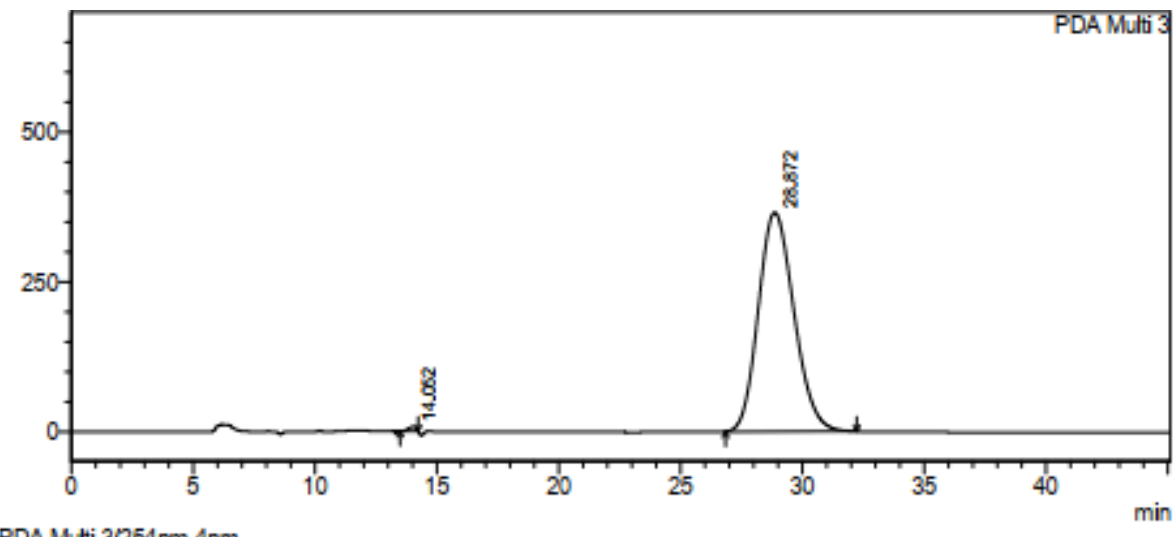

PDA Mutti $3 / 254 \mathrm{rm} 4 \mathrm{~nm}$

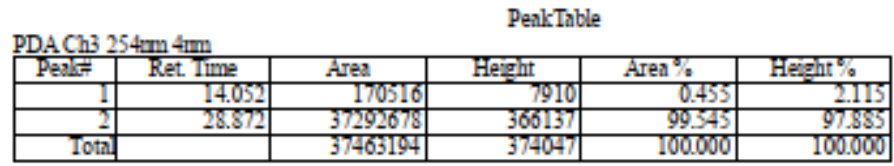

(S)-2-(3-benzamido-1-benzyl-5-methyl-2-oxoindolin-3-yl)ethane-1-sulfonyl fluoride (3g)<smiles>Cc1ccc2c(c1)[C@](CCS(=O)(=O)F)(CCS(=O)(=O)c1ccccc1)C(=O)N2Cc1ccccc1</smiles>

Petroleum ether / ethyl acetate $=8: 1$ was used as the eluent for column chromatography. White solid (45 mg, 98\%), m. p. 221.5-223.1 ${ }^{\circ} \mathrm{C} .{ }^{1} \mathbf{H}$ NMR (400 MHz, $\left.\mathrm{CDCl}_{3}\right) \delta$ 7.83-7.76 (m, 2H), 7.56-7.49 (m, 1H), 7. 45-7.39(m, 4H), 7.39-7.34 (m, 2H), 7.32-7.27 (m, 1H), 7.24 (s, 1H), 7.12 (s, 1H), 7.03 (dd, $J=$ 8.0, 0.8 Hz, 1H), 6.68 (d, $J=8.0 \mathrm{~Hz}, 1 \mathrm{H}), 5.06-4.92$ (m, 2H), 3.79-3.65 (m, 1H), 3.52-3.41(m, 1H), 2.86 (ddd, $J=14.4,9.6,5.2 \mathrm{~Hz}, 1 \mathrm{H}), 2.47$ (ddd, $J=14.4,10.0,5.6 \mathrm{~Hz}, 1 \mathrm{H}), 2.28$ (s, 3H). ${ }^{13} \mathbf{C}$ NMR (101 
$\left.\mathrm{MHz}, \mathrm{CDCl}_{3}\right) \delta 174.80,166.66,139.74,135.38,133.35,132.49,132.29,130.17,128.96,128.67,128.31$, 127.85, 127.33, 127.30, 123.98, 109.76, 59.95, 44.97 (d, $\left.J_{\mathrm{C}-\mathrm{F}}=18.1 \mathrm{~Hz}\right), 44.41,30.44,21.12 .{ }^{19} \mathbf{F}$ NMR $\left(376 \mathrm{MHz}, \mathrm{CDCl}_{3}\right) \delta$ 53.19. HRMS ESI (m/z): Calcd for $\mathrm{C}_{25} \mathrm{H}_{23} \mathrm{~N}_{2} \mathrm{O}_{4} \mathrm{FS}$, [M+Na] $]^{+}, 489.1255$; found: 489.1271. $[\alpha]_{\mathrm{D}}{ }^{20}=-4.4\left(\right.$ c $\left.0.5, \mathrm{CH}_{2} \mathrm{Cl}_{2}\right)$. Chiral HPLC analysis (Chiral pak AS-H, $i-\mathrm{PrOH} / n$-hexane $=20$ : 80 , flow rate $=0.5 \mathrm{~mL} / \mathrm{min}$, wave length $=254 \mathrm{~nm}), \mathrm{t}_{\mathrm{R}}($ minor enantiomer $)=14.919 \mathrm{~min}, \mathrm{t}_{\mathrm{R}}$ (major enantiomer) $=27.823 \mathrm{~min}, 99 \%$ ee.

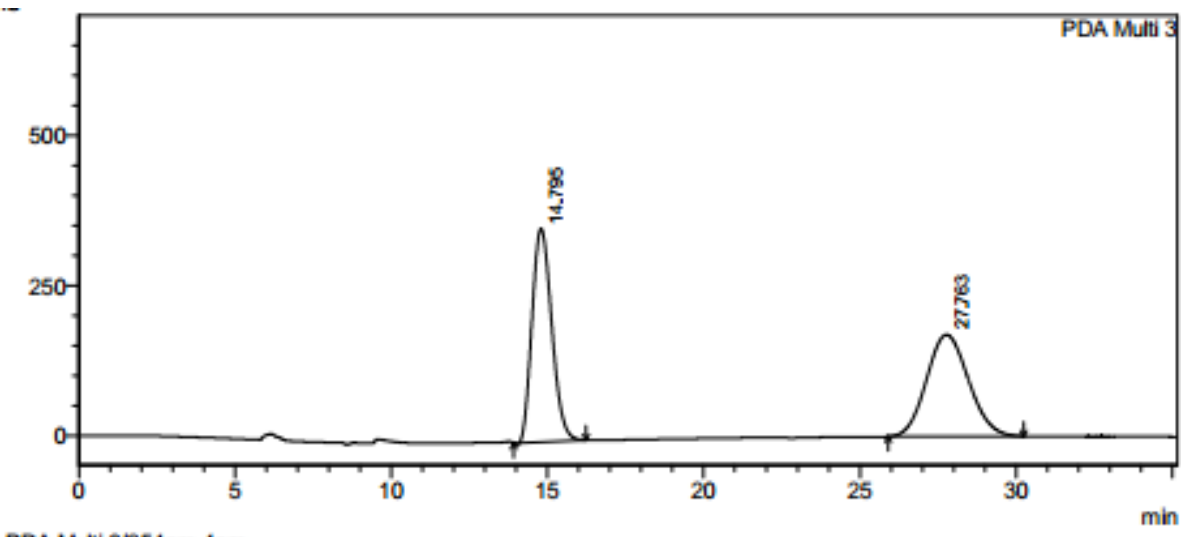

PDA Multi $3 / 254 \mathrm{~mm} 4 \mathrm{~nm}$
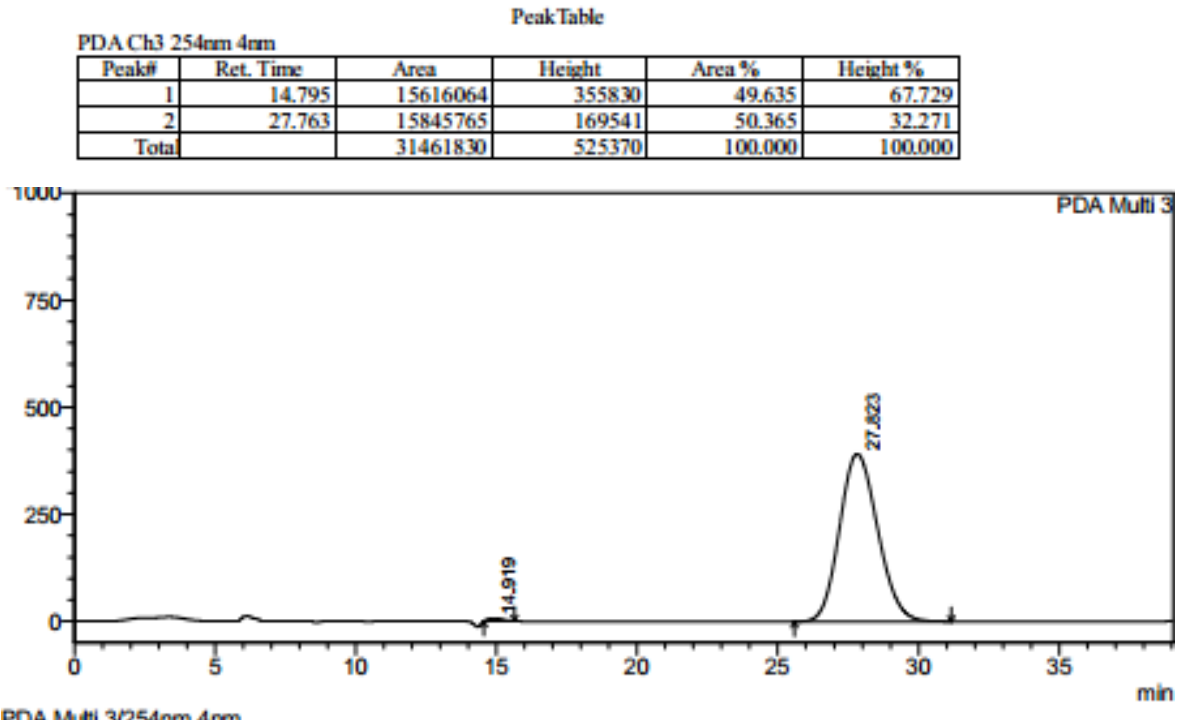

PDA Mutti $3 / 254 \mathrm{~nm} 4 \mathrm{rm}$

PDA Ch3 $254 \mathrm{~nm} 4 \mathrm{~nm}$
\begin{tabular}{|r|r|r|r|r|r|}
\hline Peakity & Ret Time & \multicolumn{1}{c|}{ Area } & Height & \multicolumn{1}{c|}{ Area $\%$} & Heinht \% \\
\hline 1 & 14.919 & 233791 & 6716 & 0.627 & 1.685 \\
\hline 2 & 27.823 & 37033738 & 391729 & 99.373 & 98.315 \\
\hline Total & & 37267530 & 398444 & 100.000 & 100000 \\
\hline
\end{tabular}

(S)-2-(3-benzamido-1-benzyl-5-methoxy-2-oxoindolin-3-yl)ethane-1-sulfonyl fluoride (3h)<smiles>COc1ccc2c(c1)[C@](CCS(=O)(=O)F)(NC(=O)c1ccccc1)C(=O)N2Cc1ccccc1</smiles>

$3 h$

Petroleum ether / ethyl acetate $=8: 1$ was used as the eluent for column chromatography. White solid (47.5 mg, 99\%), m. p. 204.6-205.6 ${ }^{\circ} \mathrm{C} .{ }^{1} \mathbf{H}$ NMR (400 MHz, $\left.\mathrm{CDCl}_{3}\right) \delta$ 7.81-7.77 (m, 2H), 7.56-7.50 (m,1H), 7.46-7.36 (m, 7H), 7.34-7.30 (m, 1H), 7.23 (s, 1H), 7.07 (d, $J=2.4 \mathrm{~Hz}, 1 \mathrm{H}), 6.77$ (dd, $J=8.8$, 
$2.4 \mathrm{~Hz}, 1 \mathrm{H}), 6.72$ (d, $J=8.4 \mathrm{~Hz}, 1 \mathrm{H}), 5.00$ (s, 2H), 3.75 (s, 3H), 3.73-3.67 (m, 1H), 3.53-3.43 (m, 1H), 2.93-2.83 (m, 1H), 2.56-2.47 (m, 1H). ${ }^{13} \mathrm{C}$ NMR (101 MHz, $\left.\mathrm{CDCl}_{3}\right) \delta 174.65,166.74,156.64,135.41$, 135.34, 132.45, 132.27, 129.66, 128.98, 128.65, 127.87, 127.34, 127.31, 113.98, 110.66, 110.52, 60.30, 55.75, 45.00 (d, JC-F = 18.3 Hz), 44.50, 30.45. ${ }^{19}$ F NMR (471 MHz, CDCl 3 ) $\delta 53.30$. HRMS ESI (m/z):

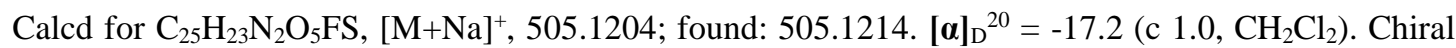
HPLC analysis (Chiral pak AS-H, $i-\mathrm{PrOH} / n$-hexane $=20: 80$, flow rate $=0.5 \mathrm{~mL} / \mathrm{min}$, wave length $=$ $254 \mathrm{~nm}), \mathrm{t}_{\mathrm{R}}($ minor enantiomer $)=8.296 \mathrm{~min}, \mathrm{t}_{\mathrm{R}}$ (major enantiomer $)=23.530 \mathrm{~min}, 98 \%$ ee .

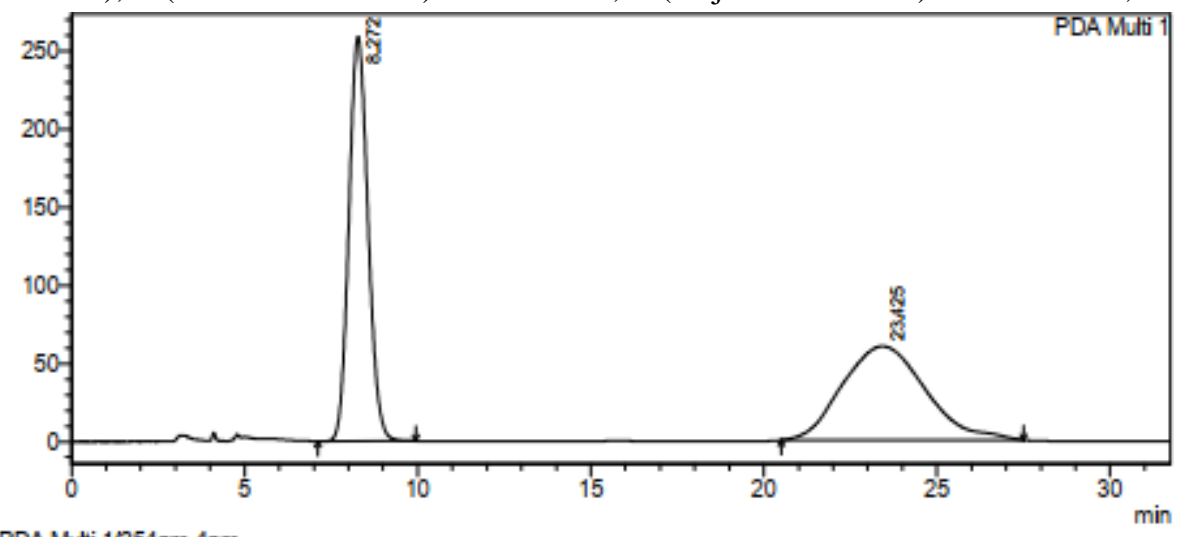

PDA Muti $1 / 254 \mathrm{~mm} 4 \mathrm{~nm}$

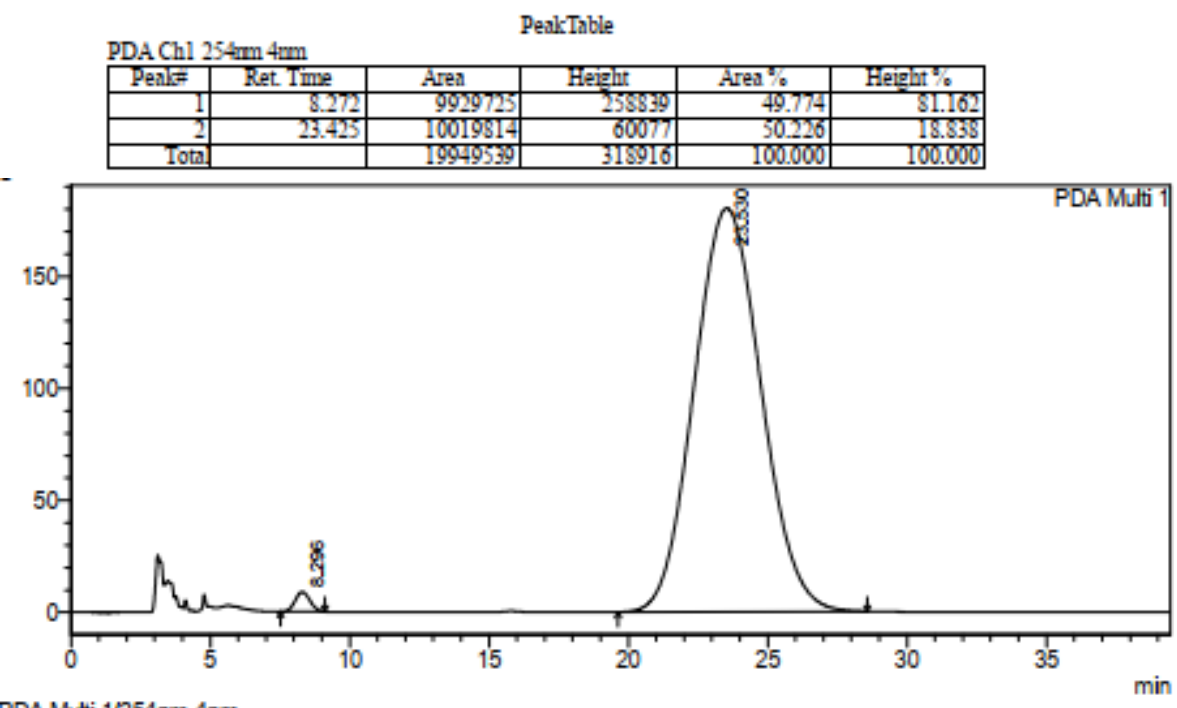

PDA Muti $1 / 254 \mathrm{rm} 4 \mathrm{~nm}$

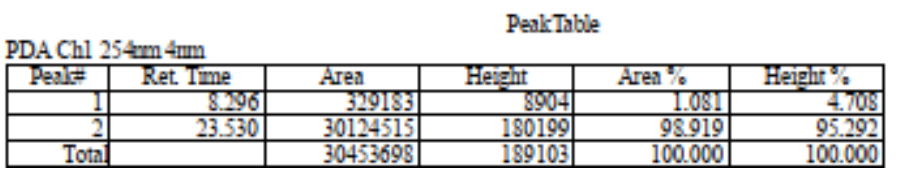

(S)-2-(3-benzamido-1-benzyl-6-fluoro-2-oxoindolin-3-yl)ethane-1-sulfonyl fluoride (3i)<smiles>O=C(N[C@]1(CCS(=O)(=O)F)C(=O)N(Cc2ccccc2)c2cc(F)ccc21)c1ccccc1</smiles>

3i 
Petroleum ether / ethyl acetate $=8: 1$ was used as the eluent for column chromatography. White solid (42.6 mg, 91\%), m. p. 241.5-242.2 ${ }^{\circ} \mathrm{C} .{ }^{1} \mathbf{H}$ NMR (400 MHz, $\left.\mathrm{CDCl}_{3}\right) \delta$ 7.81-7.74 (m, 2H), 7.55-7.50 (m, 1H), 7.44-7.29 (m, 8H), 7.27 (s, 1H), 6.73 (ddd, $J=9.2,8.4,2.4$ Hz, 1H), 6.60-6.50 (m, 1H), 4.97 (dd, $J$ $=28.0,16.0 \mathrm{~Hz}, 2 \mathrm{H}), 3.79-3.68(\mathrm{~m}, 1 \mathrm{H}), 3.54-3.43(\mathrm{~m}, 1 \mathrm{H}), 2.82$ (ddd, $J=14.8,10.0,5.6 \mathrm{~Hz}, 1 \mathrm{H}), 2.51-$ $2.40(\mathrm{~m}, 1 \mathrm{H}) .{ }^{13} \mathrm{C}$ NMR $\left(101 \mathrm{MHz}, \mathrm{CDCl}_{3}\right) \delta 175.27,166.79,163.67$ (d, JC-F $\left.=247.8 \mathrm{~Hz}\right), 143.87$ (d, JC$\mathrm{F}=11.7 \mathrm{~Hz}), 134.76,132.45,132.24,129.15,128.74,128.15,127.34,127.32,124.36$ (d, JC-F = 10.0 Hz), $123.80\left(\mathrm{~d}, J_{\mathrm{C}-\mathrm{F}}=2.9 \mathrm{~Hz}\right), 109.83\left(\mathrm{~d}, J_{\mathrm{C}-\mathrm{F}}=22.7 \mathrm{~Hz}\right), 98.96\left(\mathrm{~d}, J_{\mathrm{C}-\mathrm{F}}=27.9 \mathrm{~Hz}\right), 59.44,44.85\left(\mathrm{~d}, J_{\mathrm{C}-\mathrm{F}}=\right.$ 18.2 Hz), 44.62, 30.35, 29.72. ${ }^{19} \mathbf{F}$ NMR (376 $\left.\mathbf{M H z}, \mathrm{CDCl}_{3}\right) \delta$ 53.34. ${ }^{\mathbf{1 9}} \mathbf{F} \mathbf{~ N M R}\left(376 \mathrm{MHz}, \mathrm{CDCl}_{3}\right) \delta-$

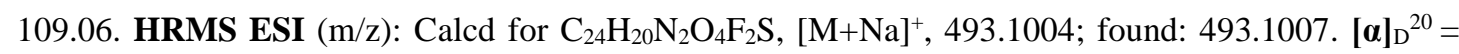
+11.4 (c 1.0, $\mathrm{CH}_{2} \mathrm{Cl}_{2}$ ). Chiral HPLC analysis (Chiral pak AS-H, $i-\mathrm{PrOH} / n$-hexane $=20: 80$, flow rate $=$ $0.5 \mathrm{~mL} / \mathrm{min}$, wave length $=254 \mathrm{~nm})$, $\mathrm{t}_{\mathrm{R}}($ minor enantiomer $)=14.657 \mathrm{~min}, \mathrm{t}_{\mathrm{R}}($ major enantiomer $)=22.642$ $\min , 99 \%$ ee.

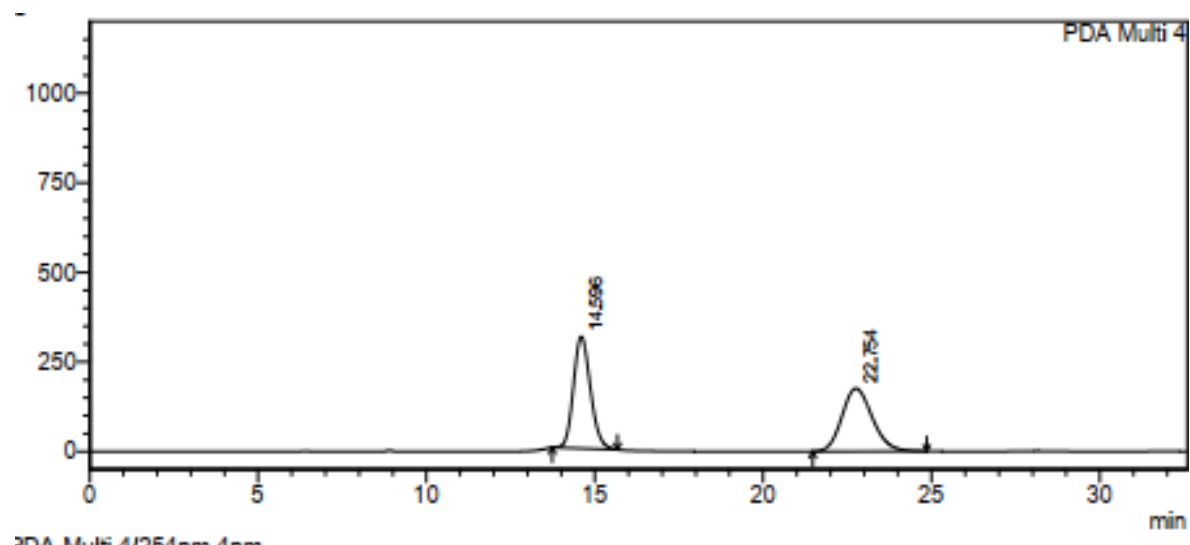

'DA Multi 4/254nm 4nm

PeakTable
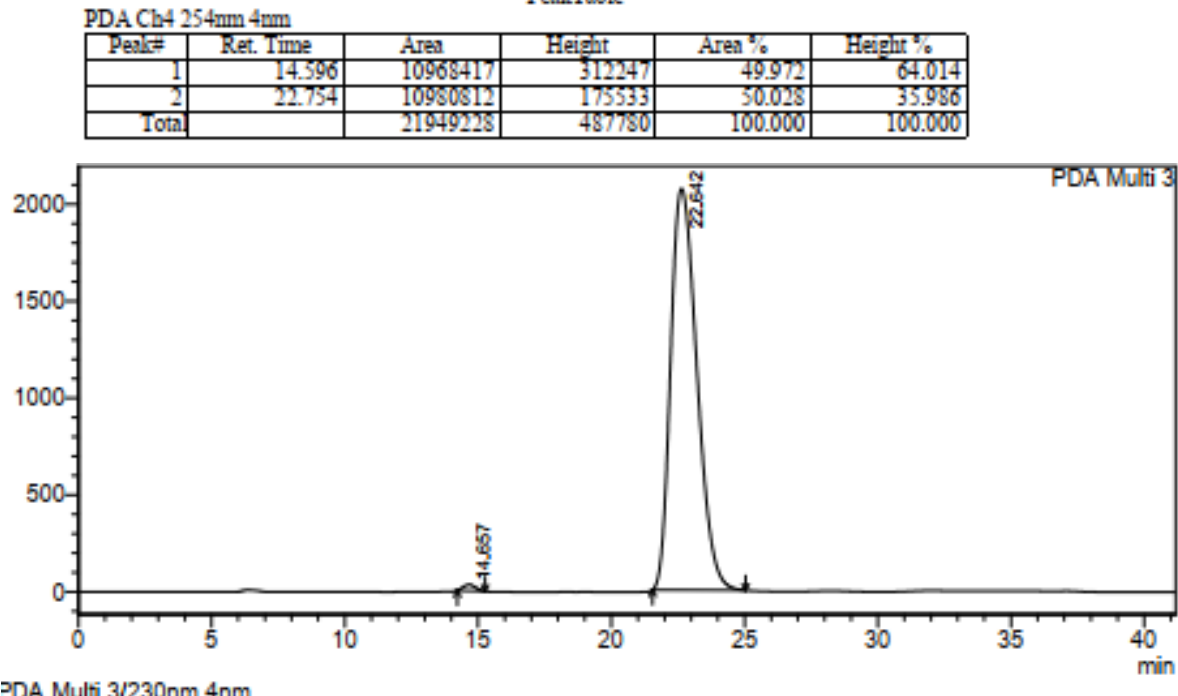

गDA Multi $3 / 230 \mathrm{~nm} 4 \mathrm{~nm}$

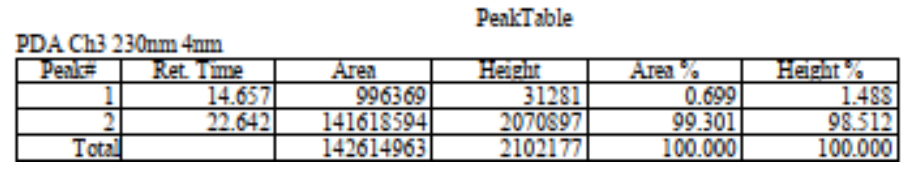

(S)-2-(3-benzamido-1-benzyl-6-chloro-2-oxoindolin-3-yl)ethane-1-sulfonyl fluoride (3j) 
<smiles>O=C(N[C@]1(CCS(=O)(=O)F)C(=O)N(Cc2ccccc2)c2cc(Cl)ccc21)c1ccccc1</smiles>

Petroleum ether / ethyl acetate $=8: 1$ was used as the eluent for column chromatography. White solid (44.8 mg, 92\%), m. p. 207.9-208.4 ${ }^{\circ} \mathrm{C} .{ }^{1} \mathbf{H}$ NMR (400 MHz, $\left.\mathrm{CDCl}_{3}\right) \delta 7.76$ (d, $\left.J=7.6 \mathrm{~Hz}, 2 \mathrm{H}\right), 7.54-$ 7.49 ( m, 1H), 7.45-7.26 (m, 9H), 7.03 (dd, $J=8.0,1.6 \mathrm{~Hz}, 1 \mathrm{H}$ ), 6.79 (d, $J=1.2 \mathrm{~Hz}, 1 \mathrm{H}$ ), 4.96 (dd, $J=$ 30.0,15.6 Hz, 2H), 3.80-3.67 (m, 1H), 3.51-3.40 (m, 1H), 2.79 (ddd, $J=14.4$, 9.6, $5.2 \mathrm{~Hz}, 1 \mathrm{H}$ ), 2.502.38 (m, 1H). ${ }^{13}$ C NMR (101 MHz, $\left.\mathrm{CDCl}_{3}\right) \delta$ 175.01, 166.78, 143.45, 135.69, 134.75, 132.49, 132.10, 129.16, 128.74, 128.16, 127.35, 127.28, 126.79, 123.95, 123.54, 110.66, 59.48, 44.78 (d, Jc-F = 18.4 Hz), 44.56, 30.22. ${ }^{19}$ F NMR (376 MHz, $\left.\mathrm{CDCl}_{3}\right) \delta$ 53.39. HRMS ESI (m/z): Calcd for $\mathrm{C}_{24} \mathrm{H}_{20} \mathrm{~N}_{2} \mathrm{O}_{4} \mathrm{FSCl}$, $[\mathrm{M}+\mathrm{Na}]^{+}$, 509.0709; found: 509.0725. $[\boldsymbol{\alpha}]_{\mathrm{D}}{ }^{20}=+22.8\left(\mathrm{c} 1.0, \mathrm{CH}_{2} \mathrm{Cl}_{2}\right.$ ). Chiral HPLC analysis (Chiral pak AS- $\mathrm{H}, i-\mathrm{PrOH} / n$-hexane $=20: 80$, flow rate $=0.5 \mathrm{~mL} / \mathrm{min}$, wave length $=254 \mathrm{~nm}$ ), $\mathrm{t}_{\mathrm{R}}$ (minor enantiomer) $=14.289 \mathrm{~min}, \mathrm{t}_{\mathrm{R}}$ (major enantiomer) $=21.853 \mathrm{~min}, 97 \%$ ee.

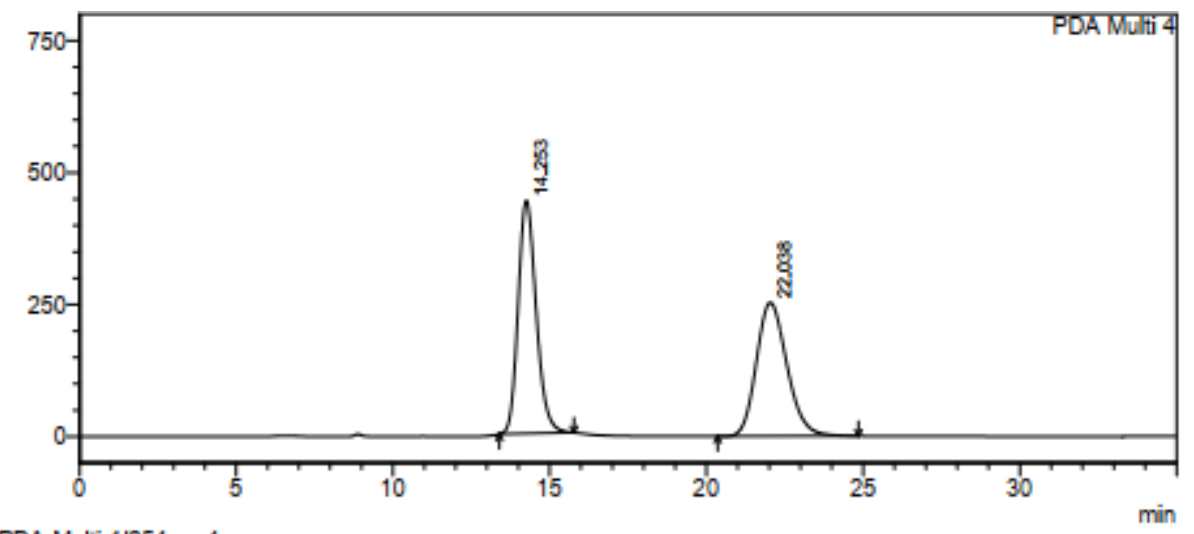

PDA Multi $4 / 254 \mathrm{~nm} 4 \mathrm{~nm}$

PeakTable

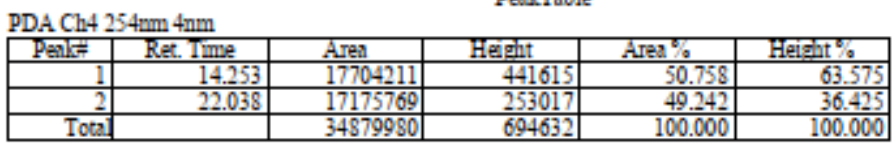




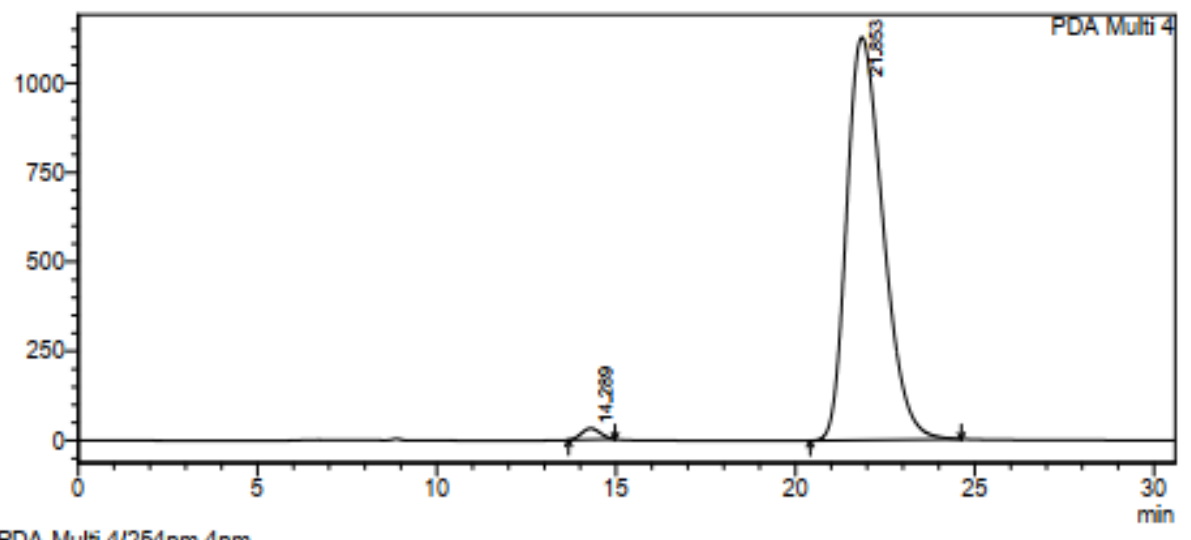

PDA Multi 4/254nm 4nm

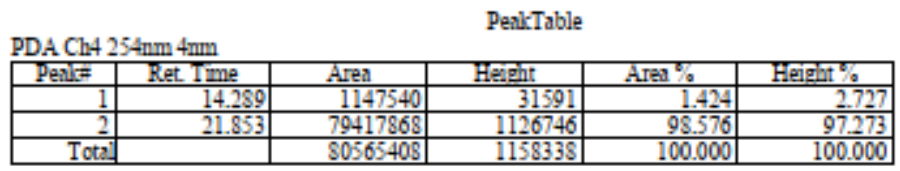

(S)-2-(3-benzamido-1-benzyl-6-bromo-2-oxoindolin-3-yl)ethane-1-sulfonyl fluoride (3k)<smiles>O=C(N[C@]1(CCS(=O)(=O)F)C(=O)N(Cc2ccccc2)c2cc(Br)ccc21)c1ccccc1</smiles>

Petroleum ether / ethyl acetate $=8: 1$ was used as the eluent for column chromatography. White solid (49 mg, 93\%), m. p. 188.7-189 ${ }^{\circ} \mathrm{C} .{ }^{1} \mathbf{H}$ NMR (400 MHz, $\left.\mathrm{CDCl}_{3}\right) \delta 7.77$ (d, $J=7.2 \mathrm{~Hz}, 2 \mathrm{H}$ ), 7.57-7.49 (m, 1H), 7.46-7.28 (m, 7H), 7.25-7.14 (m, 2H), 6.94 (d, $J=1.2 \mathrm{~Hz}, 1 \mathrm{H}$ ), 4.96 (dd, $J=30.0,16.0 \mathrm{~Hz}, 2 \mathrm{H}$ ), 3.83-3.69 (m, 1H), 3.53-3.40 (m, 1H), 2.80 (ddd, $J=14.8$, 9.6, 5.6 Hz, 1H), 2.51-2.35 (m, 1H). ${ }^{13}$ C NMR $\left(101 \mathrm{MHz}, \mathrm{CDCl}_{3}\right) \delta 174.86,166.75,143.56,134.74,132.51,132.08,129.17,128.76,128.16,127.34$, 127.27, 126.49, 124.29, 123.57, 113.38, 59.49, 44.74 (d, $\left.J_{\mathrm{C}-\mathrm{F}}=18.3 \mathrm{~Hz}\right), 44.54,30.13 .{ }^{19} \mathbf{F}$ NMR (376 $\left.\mathrm{MHz}, \mathrm{CDCl}_{3}\right) \delta$ 53.39. HRMS ESI (m/z): Calcd for $\mathrm{C}_{24} \mathrm{H}_{20} \mathrm{~N}_{2} \mathrm{O}_{4} \mathrm{FSBr}$, [M+Na] $]^{+}$553.0203; found: 553.0214. $[\boldsymbol{\alpha}]_{\mathrm{D}}{ }^{20}=+26.0\left(\right.$ c $1.0, \mathrm{CH}_{2} \mathrm{Cl}_{2}$ ). Chiral HPLC analysis (Chiral pak AS-H, $i-\mathrm{PrOH} / n$-hexane $=$ $20: 80$, flow rate $=0.5 \mathrm{~mL} / \mathrm{min}$, wave length $=254 \mathrm{~nm}), \mathrm{t}_{\mathrm{R}}$ (minor enantiomer $)=14.069 \mathrm{~min}, \mathrm{t}_{\mathrm{R}}$ (major enantiomer) $=21.708 \mathrm{~min}, 99 \%$ ee. 


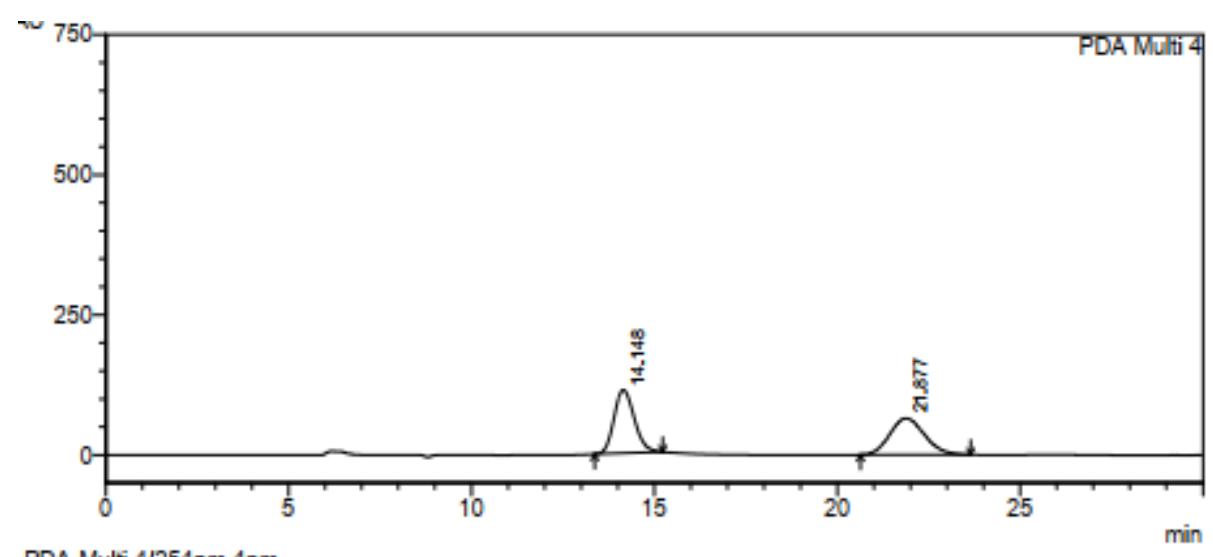

PDA Multi 4/254nm 4nm
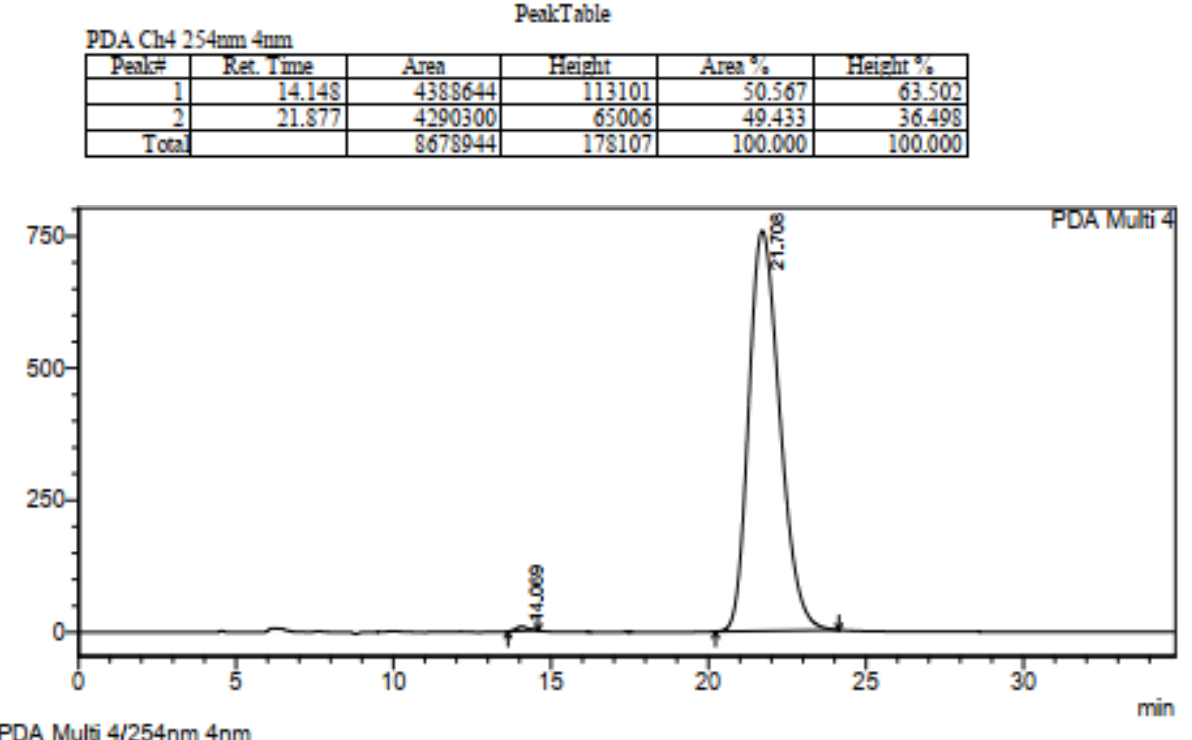

PDA Multi 4/254nm 4nm

PDA Ch4 $254 \mathrm{~nm}$ 4mm
\begin{tabular}{|r|r|r|r|r|r|}
\hline PealtF & Ret. Time & Area & Height & Aren $\%$ & Height \% \\
\hline 1 & 14.069 & 224552 & 8300 & 0.430 & 1.083 \\
\hline 2 & 21.708 & 52040373 & 757732 & 99.570 & 98.917 \\
\hline Total & & 52264925 & 765032 & 100.000 & 100.000 \\
\hline
\end{tabular}

(S)-2-(3-benzamido-1-benzyl-6-methoxy-2-oxoindolin-3-yl)ethane-1-sulfonyl fluoride (3l)<smiles>COc1ccc2c(c1)N(Cc1ccccc1)C(=O)[C@H]2CCS(=O)(=O)F</smiles>

31

Petroleum ether / ethyl acetate $=8: 1$ was used as the eluent for column chromatography. White solid (47.5 mg, 99\%), m. p. 189.4-190.4 ${ }^{\circ} \mathrm{C} .{ }^{1} \mathbf{H}$ NMR (400 MHz, $\left.\mathrm{CDCl}_{3}\right) \delta$ 7.81-7.75 (m, 2H), 7.54-7.48 (m, 1H), 7.45-7.37 ( m, 7H), 7.35-7.29 (m, 2H), 6.55 (dd, $J=8.4,2.4$ Hz, 1H), 6.41 (d, $J=2.0$ Hz, 1H), 4.97 (dd, $J=38.8,15.6$ Hz, 2H), 3.74 (s, 3H), 3.64-3.54 (m, 1H), 3.49-3.40 m, 1H), 2.86-2.77 (m, 1H), 2.57$2.48(\mathrm{~m}, 1 \mathrm{H}) .{ }^{13} \mathrm{C}$ NMR $\left(101 \mathrm{MHz}, \mathrm{CDCl}_{3}\right) \delta$ 175.35, 166.70, 161.17, 143.61, 135.25, 132.60, 132.28, 
129.03, 128.68, 127.96, 127.35, 127.28, 124.38, 120.04, 107.20, 98.11, 59.62, 55.48, $45.08\left(\mathrm{~d}, J_{\mathrm{C}-\mathrm{F}}=\right.$ $18.1 \mathrm{~Hz}), 44.46$, 30.50. ${ }^{19} \mathbf{F}$ NMR (376 $\left.\mathrm{MHz}, \mathrm{CDCl}_{3}\right) \delta$ 53.23. HRMS ESI (m/z): Calcd for

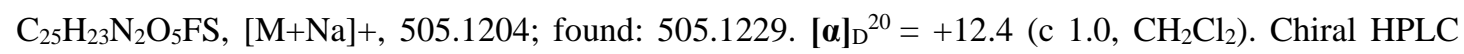
analysis (Chiral pak AS-H, $i$-PrOH $/ n$-hexane $=20: 80$, flow rate $=0.5 \mathrm{~mL} / \mathrm{min}$, wave length $=230 \mathrm{~nm}$ ), $t_{R}($ minor enantiomer $)=20.979$ min, $t_{R}($ major enantiomer $)=39.970 \mathrm{~min}$, 98\% ee.

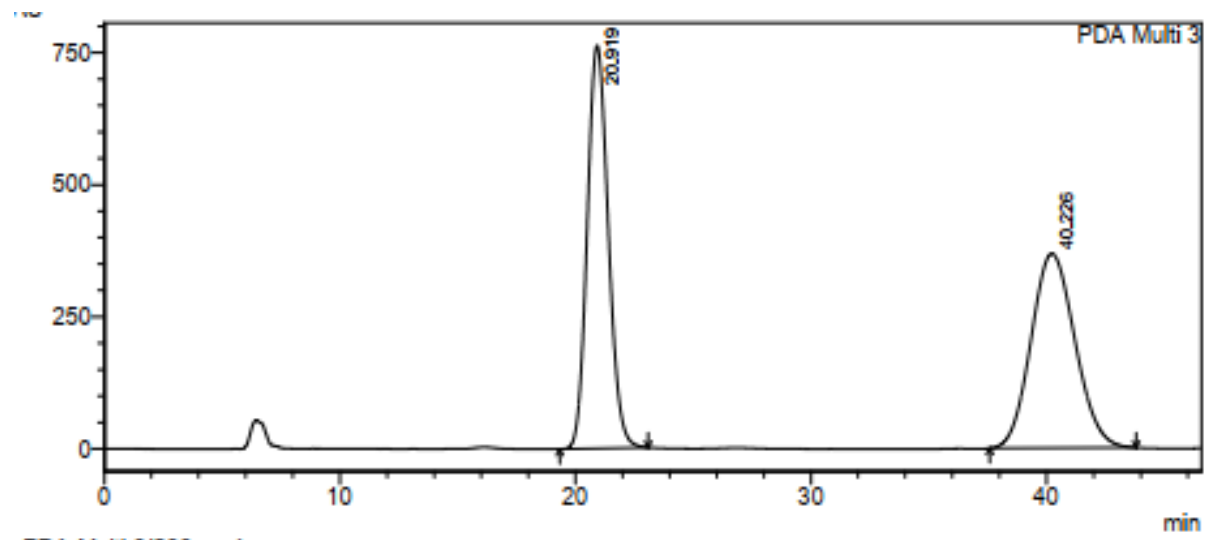

PDA Multi $3 / 230 \mathrm{~nm} 4 \mathrm{~nm}$
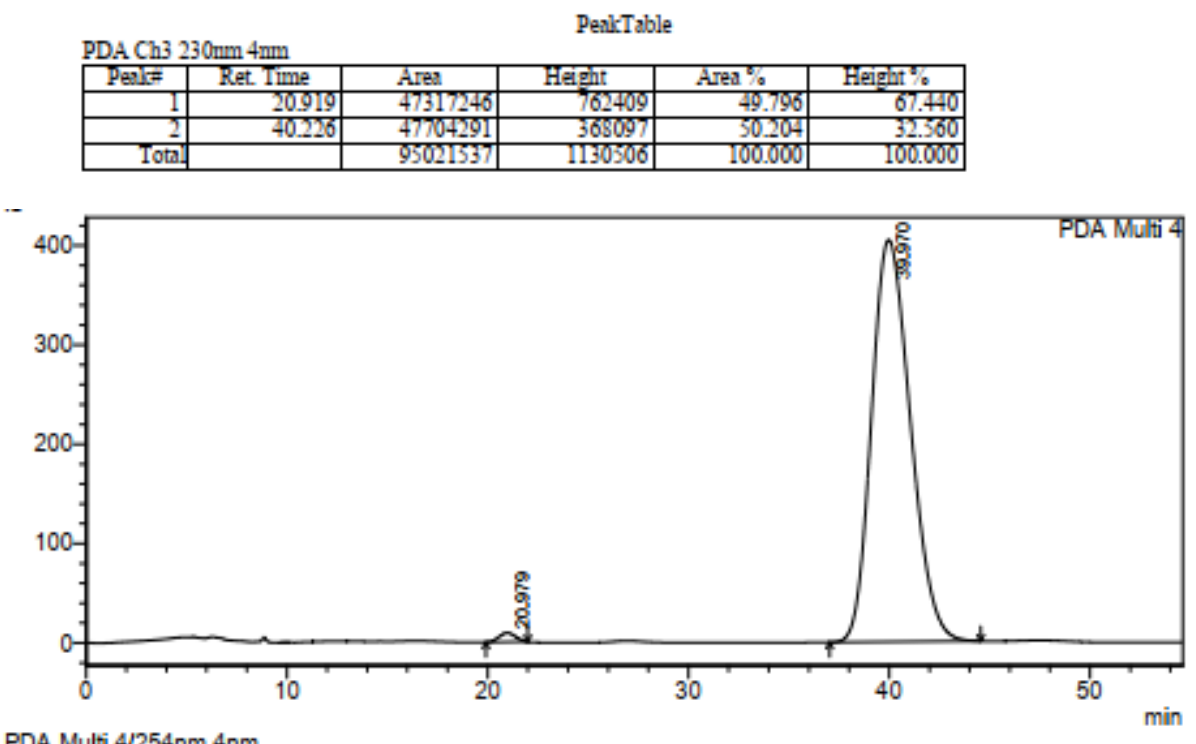

PDA Multi 4/254nm 4nm

PenikTable

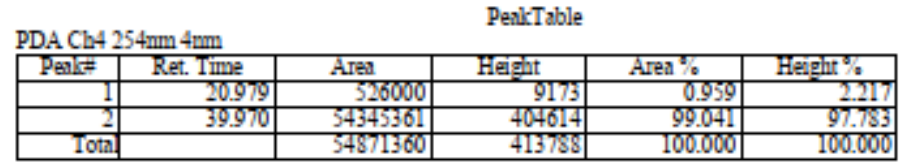

(S)-2-(3-benzamido-1-benzyl-7-fluoro-2-oxoindolin-3-yl)ethane-1-sulfonyl fluoride (3m)<smiles>O=C(N[C@]1(CCS(=O)(=O)F)C(=O)N(Cc2ccccc2)c2c(F)cccc21)c1ccccc1</smiles> 
Petroleum ether / ethyl acetate $=8: 1$ was used as the eluent for column chromatography. White solid (46.8 mg, 99\%), m. p. 187.4-188.7 ${ }^{\circ} \mathrm{C} .{ }^{1} \mathbf{H}$ NMR (400 MHz, $\left.\mathrm{CDCl}_{3}\right) \delta$ 7.82-7.71 (m, 2H), 7.54-7.48 (m, 1H), 7.46-7.32 (m, 6H), 7.33-7.26 (m, 2H), 7.18-7.09 (m, 1H), 7.07-6.97 (m, 2H), 5.13 (dd, $J=33.2,15.6$ $\mathrm{Hz}, 2 \mathrm{H}), 3.68-3.57$ (m, 1H), 3.42-3.32 (m, 1H), 2.82-2.71 (m, 1H), 2.49-2.38 (m, 1H). ${ }^{\mathbf{1 3}} \mathrm{C}$ NMR (101 $\left.\mathrm{MHz} \mathrm{CDCl}_{3}\right) \delta 174.81,166.69,147.73\left(\mathrm{~d}, J_{\mathrm{C}-\mathrm{F}}=245.9 \mathrm{~Hz}\right), 136.58,132.45,132.08,131.37\left(\mathrm{~d}, J_{\mathrm{C}-\mathrm{F}}=\right.$ $2.8 \mathrm{~Hz}), 128.83,128.72,127.92,127.53,127.35,124.42$ (d, $\left.J_{\mathrm{C}-\mathrm{F}}=6.5 \mathrm{~Hz}\right), 118.70\left(\mathrm{~d}, J_{\mathrm{C}-\mathrm{F}}=3.1 \mathrm{~Hz}\right)$, $118.15\left(\mathrm{~d}, J_{\mathrm{C}-\mathrm{F}}=19.7 \mathrm{~Hz}\right), 59.94,46.07\left(\mathrm{~d}, J_{\mathrm{C}-\mathrm{F}}=4.7 \mathrm{~Hz}\right), 44.68\left(\mathrm{~d}, J_{\mathrm{C}-\mathrm{F}}=18.4 \mathrm{~Hz}\right), 30.50 .{ }^{19} \mathbf{F}$ NMR (376 MHz, $\left.\mathrm{CDCl}_{3}\right) \delta$-132.29. ${ }^{19} \mathbf{F}$ NMR (376 MHz, $\left.\mathrm{CDCl}_{3}\right) \delta$ 53.34. HRMS ESI (m/z): Calcd for $\mathrm{C}_{24} \mathrm{H}_{20} \mathrm{~N}_{2} \mathrm{O}_{4} \mathrm{~F}_{2} \mathrm{~S}$, [M+Na] $]^{+}$, 493.1004; found: $493.1022[\alpha]_{\mathrm{D}}{ }^{20}=+18.7$ (c 1.0, $\mathrm{CH}_{2} \mathrm{Cl}_{2}$ ). Chiral HPLC analysis (Chiral pak AS-H, $i$-PrOH$/ n$-hexane $=20: 80$, flow rate $=0.5 \mathrm{~mL} / \mathrm{min}$, wave length $=254 \mathrm{~nm}$ ), $t_{\mathrm{R}}($ minor enantiomer $)=14.500 \mathrm{~min}, \mathrm{t}_{\mathrm{R}}$ (major enantiomer $)=34.242 \mathrm{~min}$, 98\% ee.

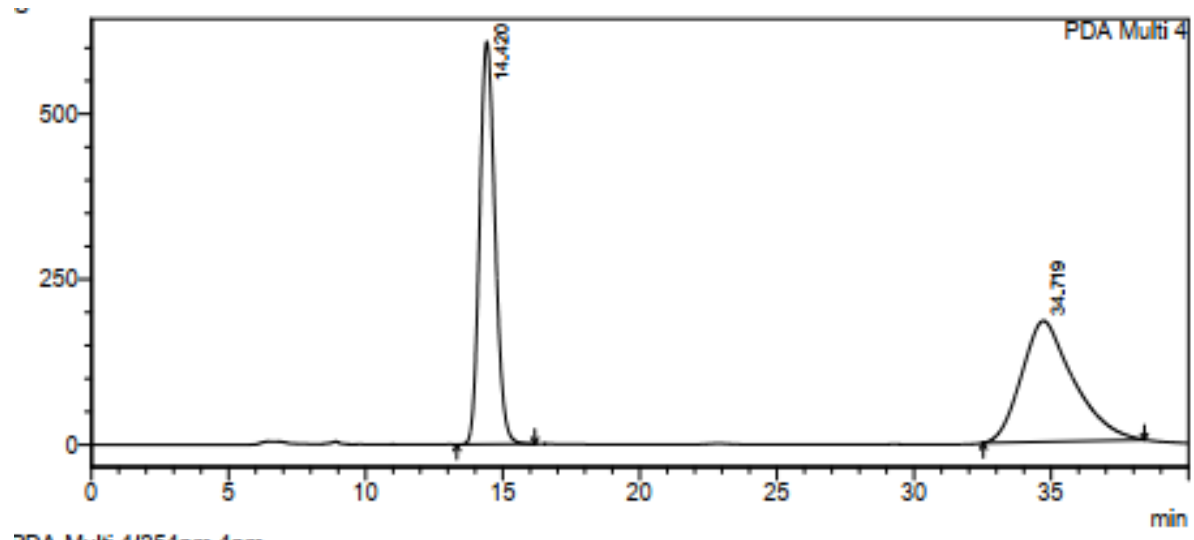

गDA Multi 4/254nm 4nm
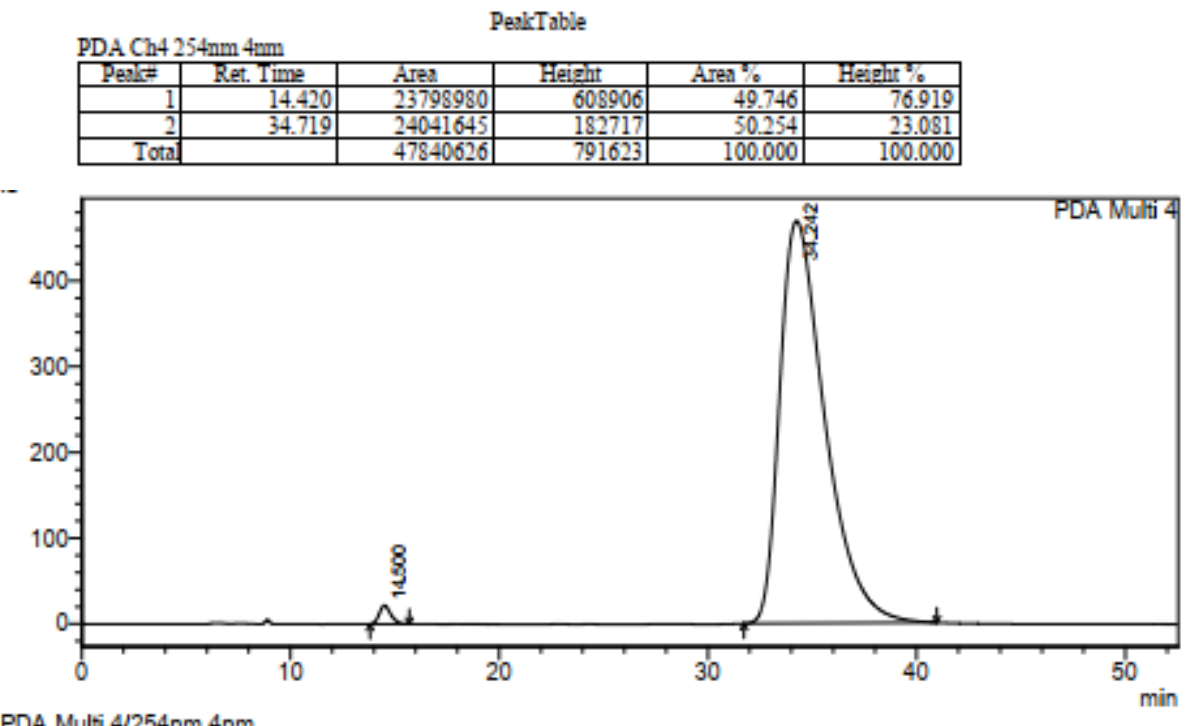

1 PDA Multi $4 / 254 \mathrm{~nm} 4 \mathrm{~nm}$

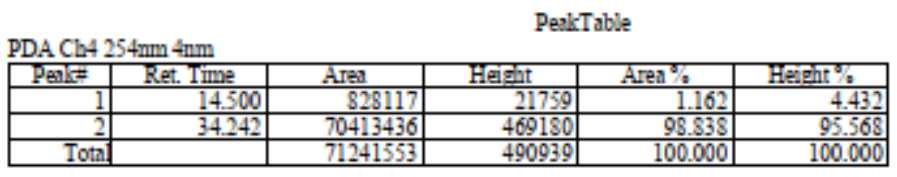

(S)-2-(3-benzamido-1-benzyl-7-chloro-2-oxoindolin-3-yl)ethane-1-sulfonyl fluoride (3n) 
<smiles>O=C(N[C@]1(CCS(=O)(=O)F)C(=O)Nc2c(Cl)cccc21)c1ccccc1</smiles>

$3 n$

Petroleum ether / ethyl acetate $=8: 1$ was used as the eluent for column chromatography. White solid (45 mg, 93\%), m. p. $177.9-178.5^{\circ} \mathrm{C} .{ }^{1} \mathbf{H}$ NMR (400 MHz, $\left.\mathrm{CDCl}_{3}\right) \delta 7.74$ (d, J = 7.2 Hz, 2H), 7.55-7.47 (m, 1H), 7.43-7.31 (m, 7H), 7.30-7.26 (m, 1H), 7.26-7.22 (m, 2H), 7.04-6.97 (m, 1H), 5.42 (dd, $J=62.4$, $16.0 \mathrm{~Hz}, 2 \mathrm{H}$ ), 3.78-3.64 (m, 1H), 3.51-3.37 (m, 1H), 2.77 (ddd, $J=14.8,9.6,5.2 \mathrm{~Hz}, 1 \mathrm{H}), 2.49-2.37$ (m, 1H). ${ }^{13} \mathrm{C}$ NMR (101 MHz, $\left.\mathrm{CDCl}_{3}\right) \delta 175.79,166.62,138.36,137.17,132.45,131.99,131.55,128.79$, 128.72, 127.46, 127.36, 126.65, 124.48, 121.23, 116.38, 59.35, 44.71 (d, $\left.J_{\mathrm{C}-\mathrm{F}}=18.2 \mathrm{~Hz}\right), 44.62,30.67$. ${ }^{19} \mathbf{F}$ NMR $\left(376 \mathrm{MHz}, \mathrm{CDCl}_{3}\right) \delta$ 53.35. HRMS ESI (m/z): Calcd for $\mathrm{C}_{24} \mathrm{H}_{20} \mathrm{~N}_{2} \mathrm{O}_{4} \mathrm{FSCl}$, $[\mathrm{M}+\mathrm{Na}]^{+}$, 509.0709; found: 509.0729. $[\boldsymbol{\alpha}]_{\mathrm{D}}{ }^{20}=+46.2\left(\mathrm{c} 1.0, \mathrm{CH}_{2} \mathrm{Cl}_{2}\right)$. Chiral HPLC analysis (Chiral pak AS-H, $i-$ $\mathrm{PrOH} / n$-hexane $=20: 80$, flow rate $=0.5 \mathrm{~mL} / \mathrm{min}$, wave length $=254 \mathrm{~nm}), \mathrm{t}_{\mathrm{R}}($ minor enantiomer $)=$ $14.718 \mathrm{~min}, \mathrm{t}_{\mathrm{R}}$ (major enantiomer) $=34.453 \mathrm{~min}, 99 \%$ ee.

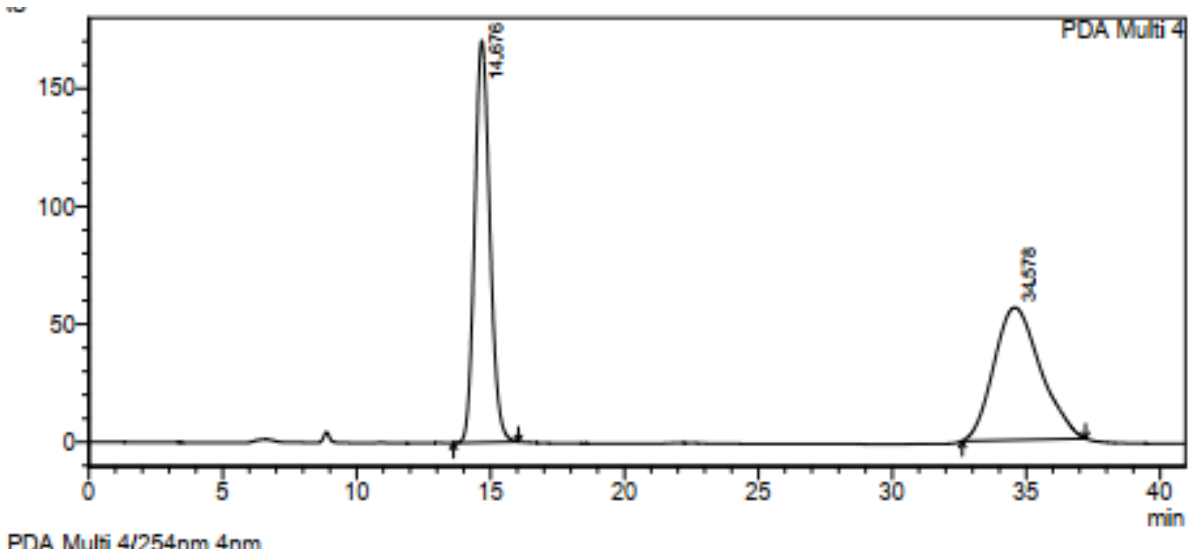

PDA Multi 4/254nm 4nm

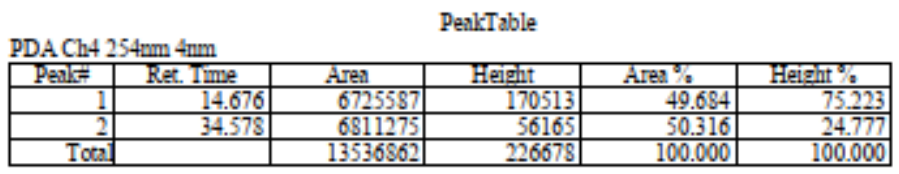




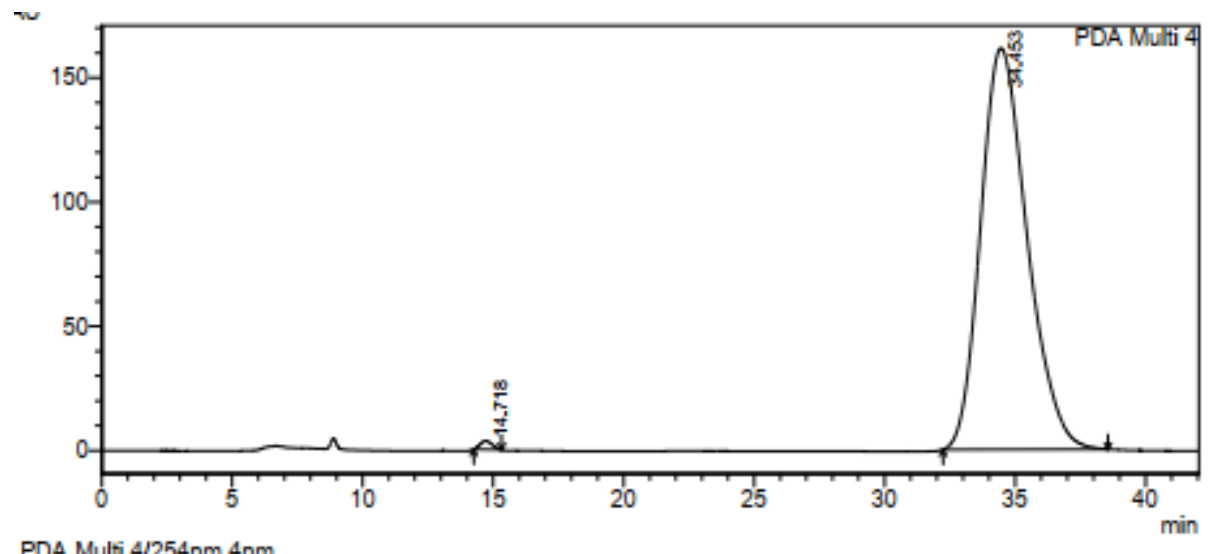

PDA Multi $4 / 254 \mathrm{~nm} 4 \mathrm{~nm}$

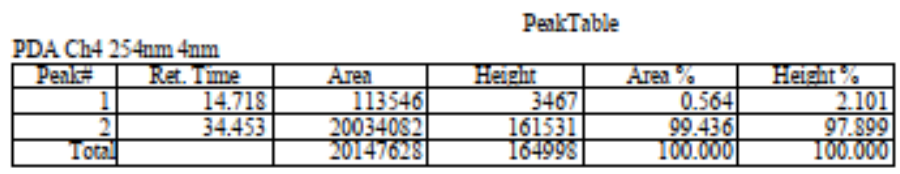

(S)-2-(3-benzamido-1-benzyl-7-bromo-2-oxoindolin-3-yl)ethane-1-sulfonyl fluoride (3o)<smiles>O=C(N[C@]1(CCS(=O)(=O)F)C(=O)Nc2c(Br)cccc21)c1ccccc1</smiles>

30

Petroleum ether / ethyl acetate $=8: 1$ was used as the eluent for column chromatography. White solid (49.1 mg, 93\%), m. p. 182.5-183.8 ${ }^{\circ} \mathrm{C} .{ }^{1} \mathbf{H}$ NMR (400 MHz, $\left.\mathrm{CDCl}_{3}\right) \delta$ 7.78-7.74 (m, 2H), 7.55-7.49 (m, 1H), 7.46-7.34 (m, 7H), 7.33-7.26 (m, 3H), 6.97-6.92 (m, $1 \mathrm{H}), 5.48$ (dd, $J=76.0,16.4 \mathrm{~Hz}, 2 \mathrm{H}), 3.80-$ 3.70 (m, 1H), 3.49-3.39 ( m, 1H), 2.79 (ddd, $J=14.4,9.2,5.2 \mathrm{~Hz}, 1 \mathrm{H}$ ), 2.42 (ddd, $J=14.8,9.2,6.0 \mathrm{~Hz}$, 1H). ${ }^{13}$ C NMR (101 MHz, $\left.\mathrm{CDCl}_{3}\right) \delta 175.84,166.56,139.81,137.12,135.81,132.46,132.02,131.91$, 128.74, 127.34, 127.31, 126.53, 124.77, 121.82, 103.37, 59.14, 45.19, 44.65 (d, $J_{\mathrm{C}-\mathrm{F}}=18.2 \mathrm{~Hz}$ ), 30.69. ${ }^{19} \mathbf{F}$ NMR (376 MHz, CDCl 3 ) $\delta$ 53.31. HRMS ESI (m/z): Calcd for $\mathrm{C}_{24} \mathrm{H}_{20} \mathrm{~N}_{2} \mathrm{O}_{4} \mathrm{FSBr},[\mathrm{M}+\mathrm{Na}]^{+}$, 553.0203; found: 553.0216. $[\boldsymbol{\alpha}]_{\mathrm{D}}^{20}=+50.8\left(\mathrm{c} 1.0, \mathrm{CH}_{2} \mathrm{Cl}_{2}\right.$ ). Chiral HPLC analysis (Chiral pak AS-H, $i$ $\mathrm{PrOH} / n$-hexane $=20: 80$, flow rate $=0.5 \mathrm{~mL} / \mathrm{min}$, wave length $=220 \mathrm{~nm}), \mathrm{t}_{\mathrm{R}}($ minor enantiomer $)=$ $19.223 \mathrm{~min}, \mathrm{t}_{\mathrm{R}}$ (major enantiomer) $=50.930 \mathrm{~min}, 98 \%$ ee. 


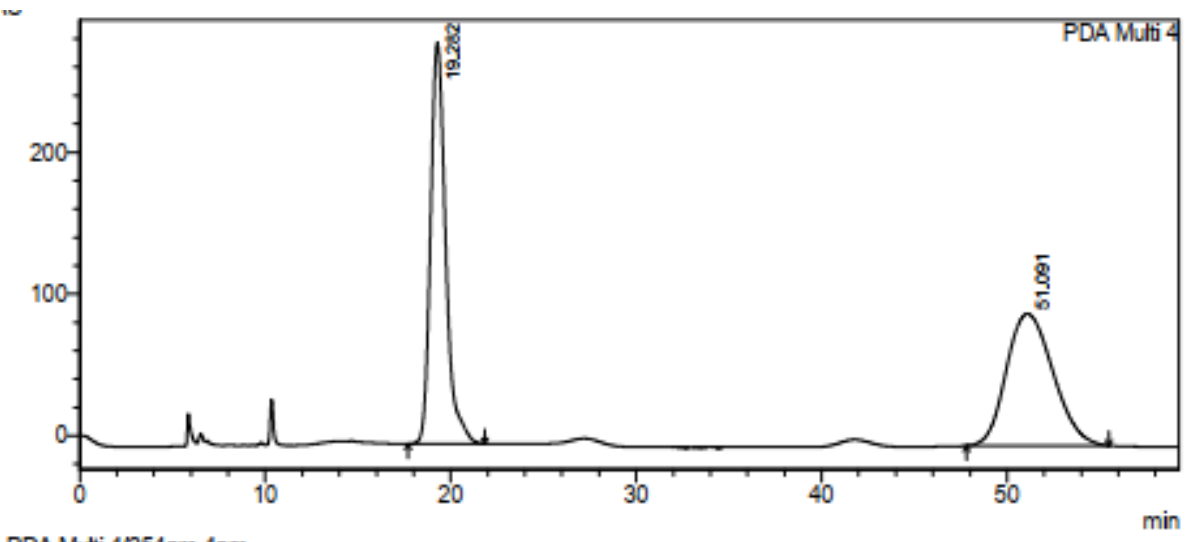

PDA Muti $4 / 254 \mathrm{rm} 4 \mathrm{~nm}$
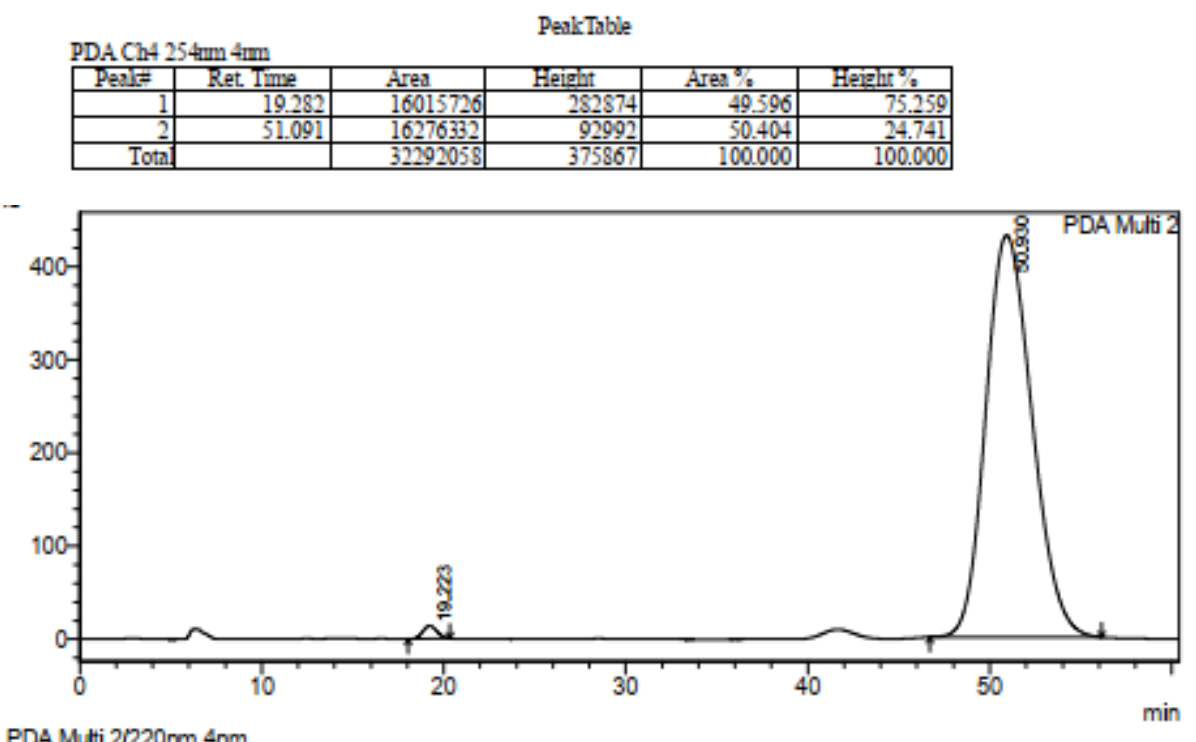

PDA Multi $2 / 220 \mathrm{rm} 4 \mathrm{~nm}$

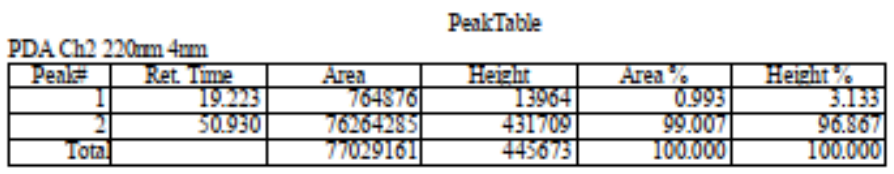

(S)-2-(3-benzamido-1-benzyl-2-oxo-7-(trifluoromethyl)indolin-3-yl)ethane-1-sulfonyl fluoride (3p)<smiles>O=C(N[C@]1(CCS(=O)(=O)F)C(=O)N(Cc2ccccc2)c2c(C(F)(F)F)cccc21)c1ccccc1</smiles>

$3 p$

Petroleum ether / ethyl acetate $=8: 1$ was used as the eluent for column chromatography. White solid (41 mg, 86\%), m. p. 211.6-212.3 ${ }^{\circ} \mathrm{C} .{ }^{1} \mathbf{H}$ NMR (400 MHz, $\left.\mathrm{CDCl}_{3}\right) \delta$ 7.76-7.67 (m, 2H), 7.62 (d, $J=8.0$ $\mathrm{Hz}, 1 \mathrm{H}), 7.53-7.44(\mathrm{~m}, 3 \mathrm{H}), 7.42-7.21$ (m, 7H), 7.18-7.11 (m, 1H), 5.27-5.16 (m, 2H), 3.75-3.62(m, $1 \mathrm{H})$, 3.50 -3.39 (m, 1H), 2.81-2.69 (m, 1H), 2.46-2.33 (m, 1H). ${ }^{13}$ C NMR (101 MHz, $\left.\mathrm{CDCl}_{3}\right) \delta$ 176.62, 166.77, 140.54, 136.05, 132.54, 131.88, 131.76, 128.75, 128.58, 127.98 (d, $\left.J_{\mathrm{C}-\mathrm{F}}=6.1 \mathrm{~Hz}\right), 127.37,127.24,126.27$, 
126.01, 123.15, 113.63 (d, $\left.J_{\mathrm{C}-\mathrm{F}}=32.9 \mathrm{~Hz}\right), 58.11,46.56$ (d, $\left.J_{\mathrm{C}-\mathrm{F}}=4.5 \mathrm{~Hz}\right), 44.59$ (d, $\left.J_{\mathrm{C}-\mathrm{F}}=18.1 \mathrm{~Hz}\right)$, 30.51. ${ }^{19}$ F NMR (376 MHz, $\left.\mathrm{CDCl}_{3}\right) \delta$ 53.47, -54.62. HRMS ESI (m/z): Calcd for $\mathrm{C}_{25} \mathrm{H}_{20} \mathrm{~N}_{2} \mathrm{O}_{4} \mathrm{~F}_{4} \mathrm{~S}$, $[\mathrm{M}+\mathrm{Na}]^{+}$, 543.0972; found: 543.0988. $[\alpha]_{\mathrm{D}}{ }^{20}=+45.9$ (c 1.0, $\mathrm{CH}_{2} \mathrm{Cl}_{2}$ ). Chiral HPLC analysis (Chiral pak AS-H, $i-\mathrm{PrOH} / n$-hexane $=20: 80$, flow rate $=0.5 \mathrm{~mL} / \mathrm{min}$, wave length $=254 \mathrm{~nm}), \mathrm{t}_{\mathrm{R}}$ (minor enantiomer) $=10.377 \mathrm{~min}, \mathrm{t}_{\mathrm{R}}$ (major enantiomer $)=22.496 \mathrm{~min}, 98 \%$ ee.

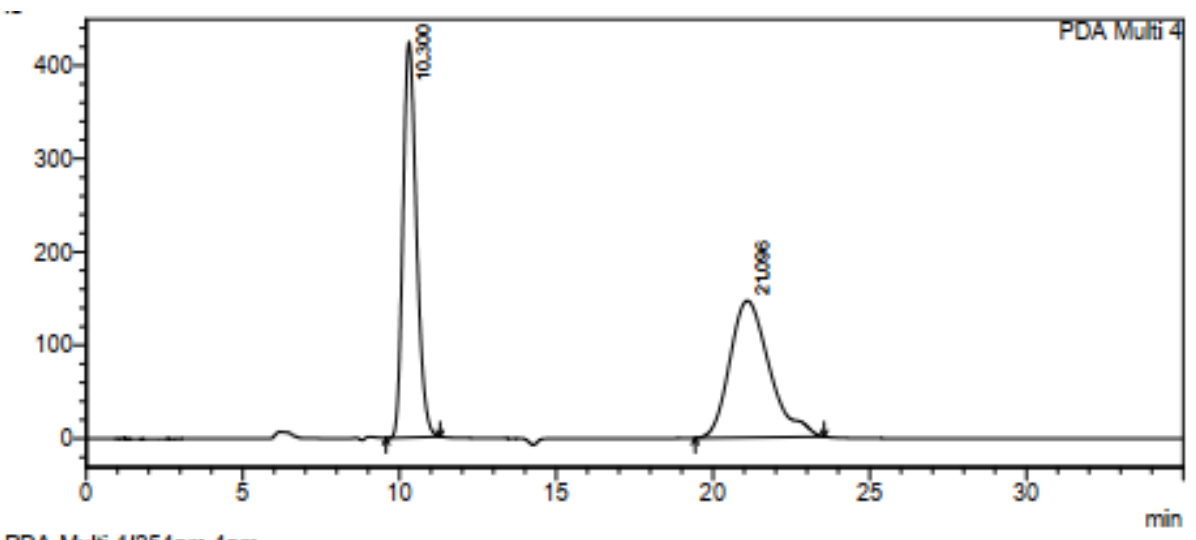

PDA Multi 4/254nm 4nm
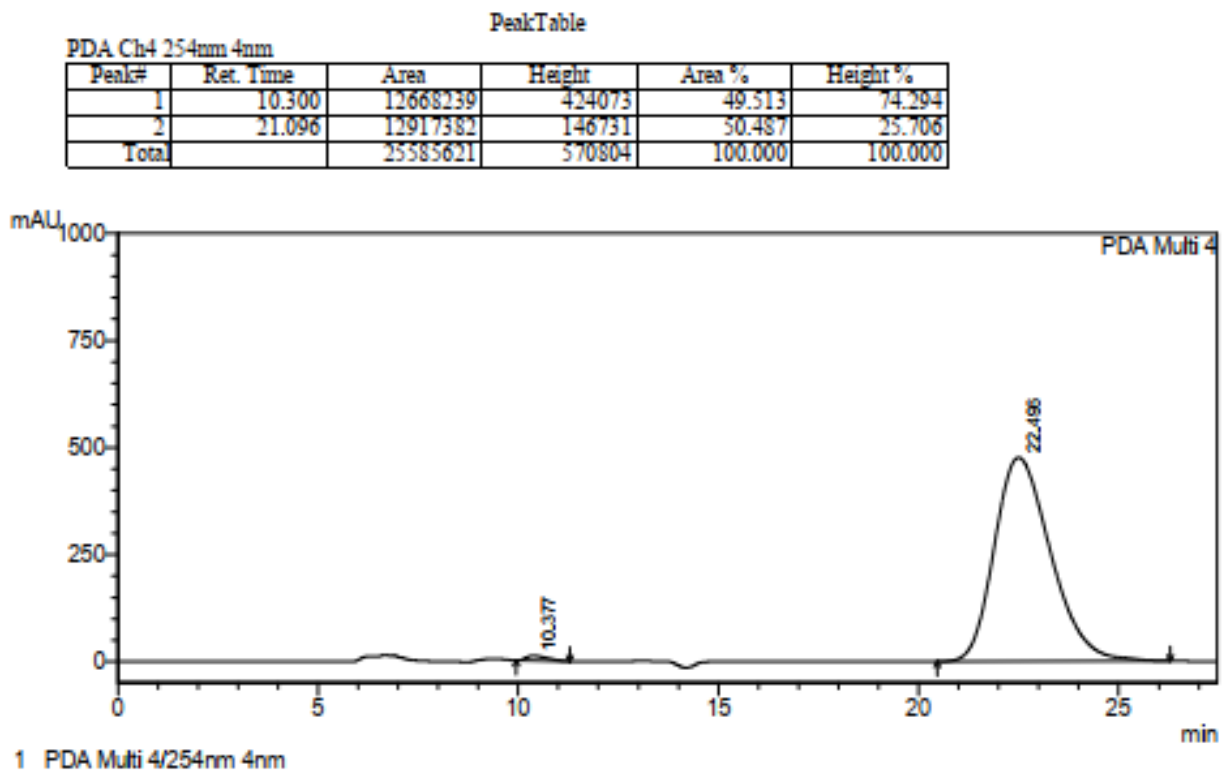

1 PDA Mutti $4 / 254 \mathrm{~mm} 4 \mathrm{~nm}$

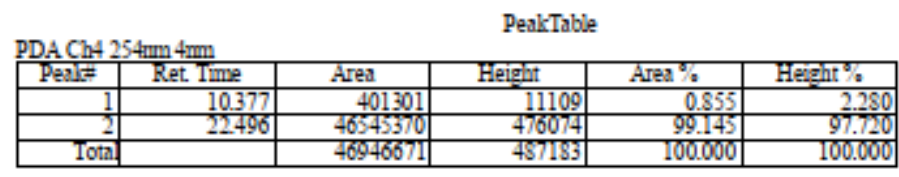

(S)-2-(3-benzamido-1-benzyl-5,7-dimethyl-2-oxoindolin-3-yl)ethane-1-sulfonyl fluoride (3q)<smiles>Cc1cc(C)c2c(c1)[C@](CCS(=O)(=O)F)(NC(=O)c1ccccc1)C(=O)N2Cc1ccccc1</smiles>

$3 q$ 
Petroleum ether / ethyl acetate $=8: 1$ was used as the eluent for column chromatography. White solid (36.6 mg, 83\%), m. p. 220.4-221.7 ${ }^{\circ} \mathrm{C}^{1}{ }^{1} \mathrm{H}$ NMR (500 MHz, $\left.\mathrm{CDCl}_{3}\right) \delta 7.79$ (d, $\left.J=7.5 \mathrm{~Hz}, 2 \mathrm{H}\right), 7.54-$ 7.48( m, 1H), 7.43-7.26 (m, 8H), 7.03 (s, 1H), 6.82 (s, 1H), 5.23 (dd, $J=68.5,17.0 \mathrm{~Hz}, 2 \mathrm{H}$ ), 3.81-3.72 (m, 1H), 3.54-3.46 (m, 1H), 2.84-2.76 (m, 1H), 2.49-2.41 (m, 1H), 2.24 (s, 6H). ${ }^{13} \mathrm{C}$ NMR (126 MHz, $\left.\mathrm{CDCl}_{3}\right) \delta 175.92,166.52,137.78,137.35,134.35,133.30,132.47,132.23,129.35,129.02,128.62$, 127.38, 127.34, 125.85, 121.37, 120.36, 59.37, 45.64, 45.01 (d, $J_{\mathrm{C}-\mathrm{F}}=17.9 \mathrm{~Hz}$ ), 30.93, 20.80, 18.64. ${ }^{19} \mathbf{F}$ NMR (471 MHz, $\left.\mathrm{CDCl}_{3}\right) \delta$ 53.19. HRMS (m/z): Calcd for $\mathrm{C}_{26} \mathrm{H}_{25} \mathrm{~N}_{2} \mathrm{O}_{4} \mathrm{FS},[\mathrm{M}+\mathrm{Na}]^{+}, 503.1411$, found:503.1428. $[\boldsymbol{\alpha}]_{\mathrm{D}}^{20}=-12.3\left(\mathrm{c} 1.0, \mathrm{CH}_{2} \mathrm{Cl}_{2}\right)$. Chiral HPLC analysis (Chiral pak AS-H, $i-\mathrm{PrOH} / n-$ hexane $=20: 80$, flow rate $=0.5 \mathrm{~mL} / \mathrm{min}$, wave length $=254 \mathrm{~nm}), \mathrm{t}_{\mathrm{R}}($ minor enantiomer $)=14.327 \mathrm{~min}$, $t_{R}($ major enantiomer $)=32.837 \mathrm{~min}, 99 \%$ ee.

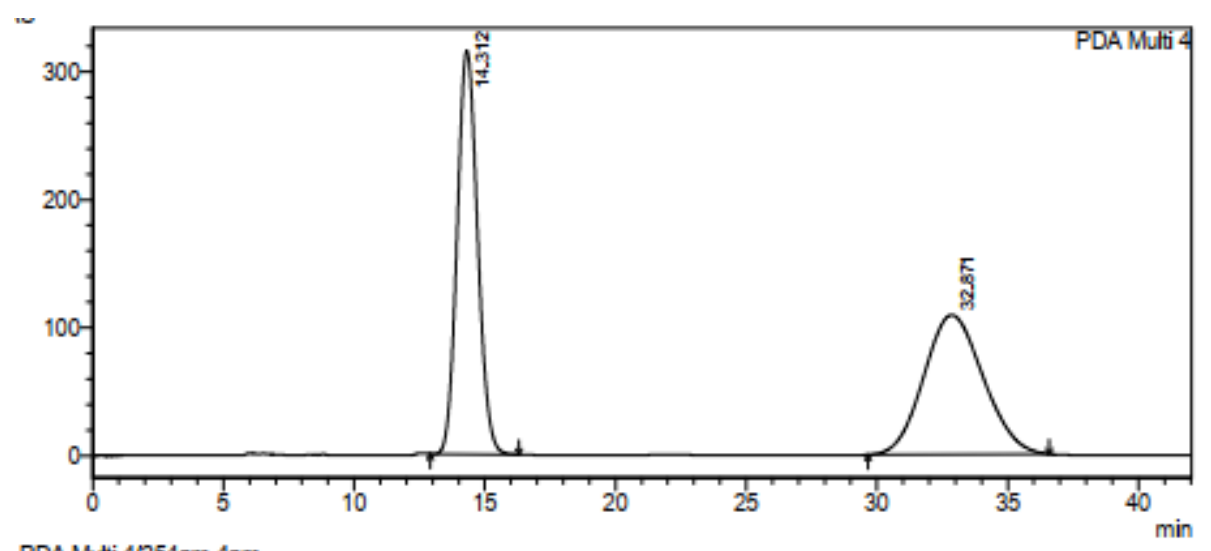

PDA Muti $4 / 254 \mathrm{~mm} 4 \mathrm{~nm}$
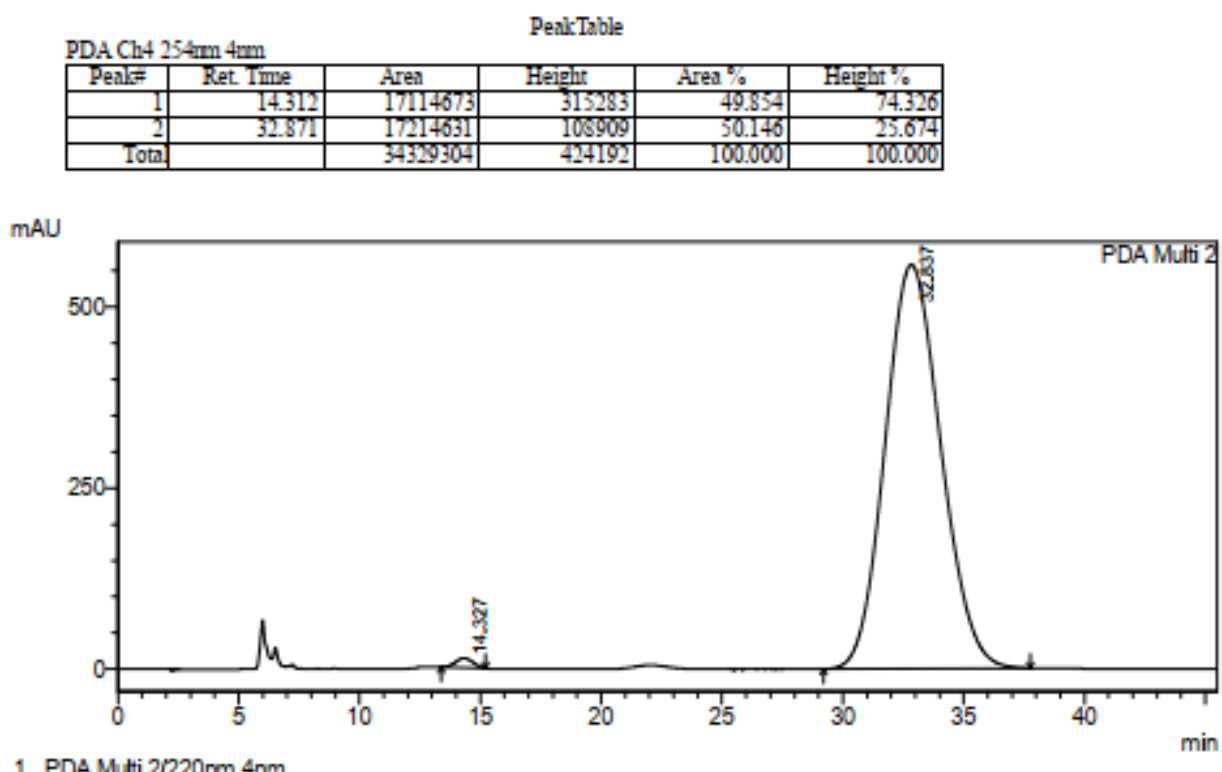

1 PDA Mutti $2 / 220 \mathrm{~mm} 4 \mathrm{~nm}$

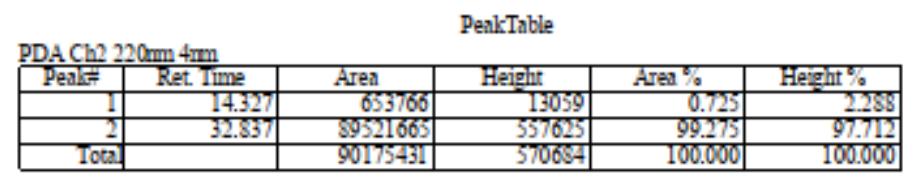

(S)-2-(3-(2-naphthamido)-1-benzyl-2-oxoindolin-3-yl)ethane-1-sulfonyl fluoride (3r) 
<smiles>O=C(N[C@]1(CCS(=O)(=O)F)C(=O)N(Cc2ccccc2)c2ccccc21)c1ccc2ccccc2c1</smiles>

Petroleum ether / ethyl acetate $=6: 1$ was used as the eluent for column chromatography. White solid (47.6 mg, 95\%), m. p. 228.9-230.4 ${ }^{\circ} \mathrm{C} .{ }^{1} \mathbf{H}$ NMR (400 MHz, $\left.\mathrm{CDCl}_{3}\right) \delta 8.23$ (s, 1H), 7.85-7.72 (m, 4H), 7.58-7.48 (m, 3H), 7.46-7.36 (m, 5H), 7.34-7.28 (m, 1H), 7.27-7.21 (m, 1H), 7.06 (td, $J=7.6,0.8 \mathrm{~Hz}$, $1 \mathrm{H}), 6.81$ (d, $J=8.0 \mathrm{~Hz}, 1 \mathrm{H}), 5.03$ (q, $J=15.7 \mathrm{~Hz}, 2 \mathrm{H}), 3.79-3.67(\mathrm{~m}, 1 \mathrm{H}), 3.54-3.43(\mathrm{~m}, 1 \mathrm{H}), 2.95-2.84$ (m, 1H), 2.63-2.51(m, 1H). ${ }^{13} \mathrm{C}$ NMR (101 MHz, $\left.\mathrm{CDCl}_{3}\right) \delta$ 175.22, 166.79, 142.25, 135.31, 135.00, 132.43, 129.84, 129.45, 129.17, 129.05, 128.51, 128.39, 128.32, 128.06, 127.94, 127.71, 127.34, 126.86, 123.67, 123.43, 123.06, 110.07, 60.13, 45.06 (d, $\left.J_{\mathrm{C}-\mathrm{F}}=18.1 \mathrm{~Hz}\right), 44.47,30.49 .{ }^{19}$ F NMR (376 MHz, $\left.\mathrm{CDCl}_{3}\right) \delta$ 53.36. HRMS ESI (m/z): Calcd for $\mathrm{C}_{28} \mathrm{H}_{23} \mathrm{~N}_{2} \mathrm{O}_{4} \mathrm{FS}$, [M+Na] ${ }^{+}$, 525.1255; found: 525.1271. $[\alpha]_{\mathrm{D}}{ }^{20}=+24.8\left(\mathrm{c} 1.0, \mathrm{CH}_{2} \mathrm{Cl}_{2}\right.$ ). Chiral HPLC analysis (Chiral pak AS-H, $i-\mathrm{PrOH} / n$-hexane $=20: 80$, flow rate $=0.5 \mathrm{~mL} / \mathrm{min}$, wave length $=254 \mathrm{~nm}), \mathrm{t}_{\mathrm{R}}($ minor enantiomer $)=18.755 \mathrm{~min}, \mathrm{t}_{\mathrm{R}}($ major enantiomer $)=$ $44.439 \mathrm{~min}, 99 \%$ ee.

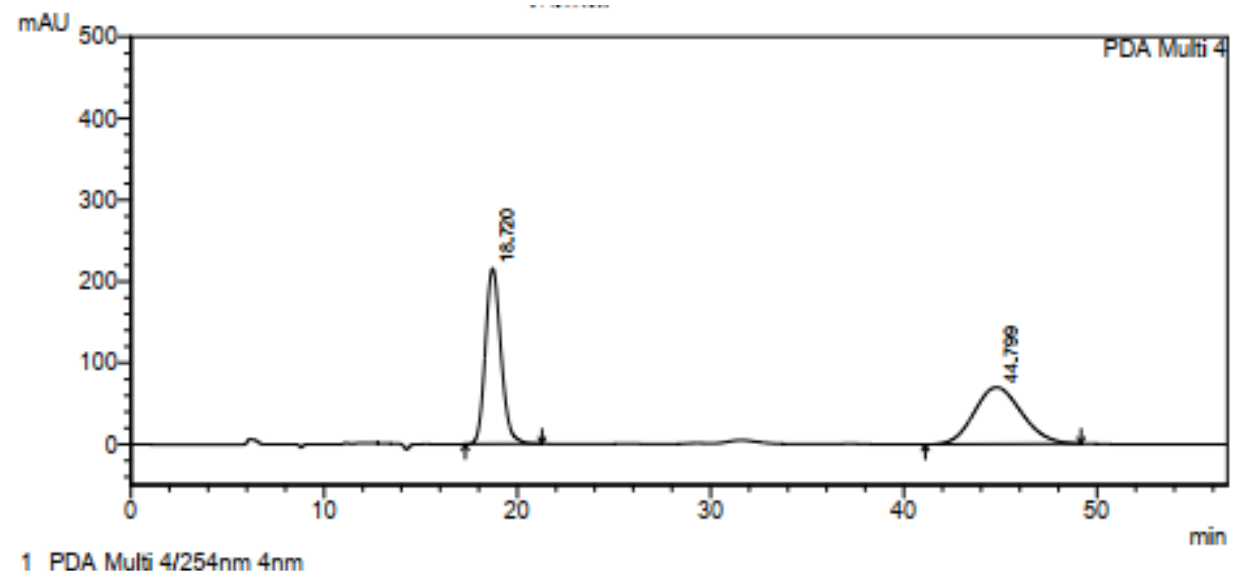

1 PDA Multi 4/254nm 4nm

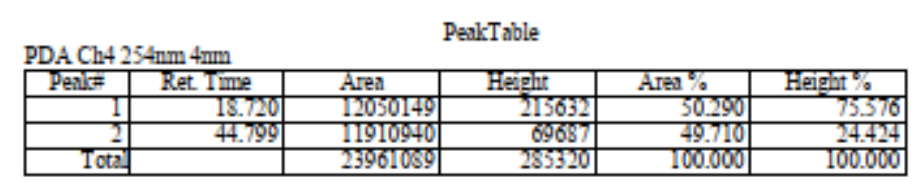




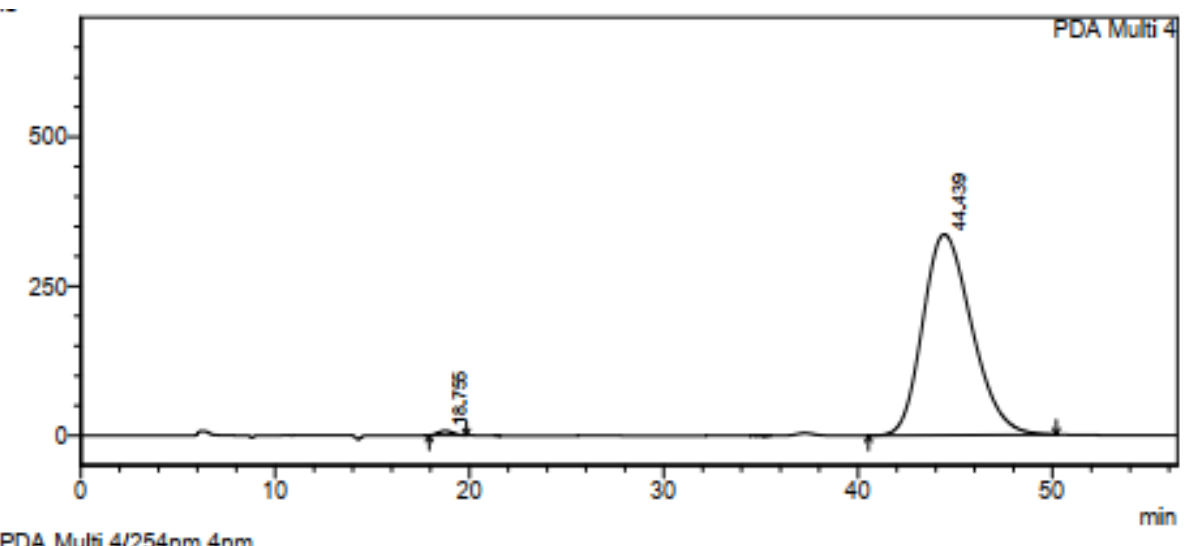

PDA Multi 4/254nm 4nm

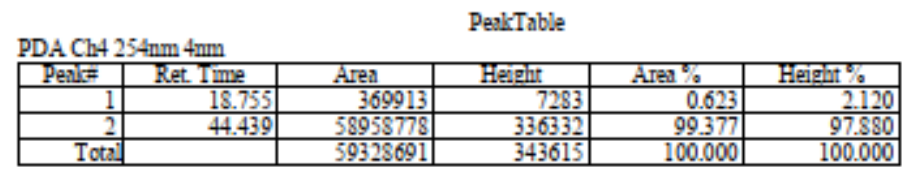

(S)-2-(1-benzyl-3-(1H-indole-3-carboxamido)-2-oxoindolin-3-yl)ethane-1-sulfonyl fluoride (3s)<smiles>O=C(N[C@]1(CCS(=O)(=O)OF)C(=O)Nc2ccccc21)c1c[nH]c2ccccc12</smiles>

Petroleum ether / ethyl acetate $=6: 1$ was used as the eluent for column chromatography. White solid (43.9 mg, 90\%), m. p. 217.9-218.6 ${ }^{\circ} \mathrm{C} .{ }^{1} \mathbf{H}$ NMR (500 MHz, $\left.\mathrm{CDCl}_{3}\right) \delta 8.51$ (d, J = 2.0 Hz, 1H), 7.99 (d, $J=7.5 \mathrm{~Hz}, 1 \mathrm{H}$ ), 7.59 (d, $J=3.0 \mathrm{~Hz}, 1 \mathrm{H}), 7.45$ (d, $J=7.4 \mathrm{~Hz}, 2 \mathrm{H}), 7.37-7.32$ (m, 2H), 7.31-7.25 (m, 4H), $7.23(\mathrm{td}, J=8.0,1.0 \mathrm{~Hz}, 1 \mathrm{H}), 7.20-7.11(\mathrm{~m}, 2 \mathrm{H}), 7.05-7.01$ (m, 1H), 6.98 (d, $J=8.0 \mathrm{~Hz}, 1 \mathrm{H}), 6.81$ (d, $J$ $=8.0 \mathrm{~Hz}, 1 \mathrm{H}), 5.03(\mathrm{dd}, J=39.0,16.0 \mathrm{~Hz}, 2 \mathrm{H}), 3.70-3.61(\mathrm{~m}, 1 \mathrm{H}), 3.51-3.43(\mathrm{~m}, 1 \mathrm{H}), 2.78$ (ddd, $J=$ 14.0, 11.5, $5.0 \mathrm{~Hz}, 1 \mathrm{H}), 2.55$ (ddd, $J=14.011 .5,4.5 \mathrm{~Hz}, 1 \mathrm{H}) .{ }^{13} \mathrm{C}$ NMR $\left(126 \mathrm{MHz}, \mathrm{CDCl}_{3}\right) \delta 176.23$, 164.94, 141.99, 135.96, 135.25, 129.61, 129.02, 128.88, 128.77, 127.93, 127.23, 125.04, 123.71, 123.02, 122.91, 121.80, 120.26, 111.95, 110.05, 109.77, 60.10, 45.20 (d, $\left.J_{\mathrm{C}-\mathrm{F}}=18.1 \mathrm{~Hz}\right), 44.47,30.74 .{ }^{19} \mathbf{F}$ NMR $\left(471 \mathrm{MHz}, \mathrm{CDCl}_{3}\right) \delta$ 53.38. HRMS ESI (m/z): Calcd for $\mathrm{C}_{26} \mathrm{H}_{22} \mathrm{~N}_{3} \mathrm{O}_{4} \mathrm{FS}$, $[\mathrm{M}+\mathrm{H}]^{+}$, 492.1388; found: 492.1401. $[\alpha]_{\mathrm{D}}{ }^{20}=-4.3$ (c 1.0, $\mathrm{CH}_{2} \mathrm{Cl}_{2}$ ). Chiral HPLC analysis (Chiral pak AS-H, $i-\mathrm{PrOH} / n$-hexane $=20$ : 80, flow rate $=0.5 \mathrm{~mL} / \mathrm{min}$, wave length $=254 \mathrm{~nm}$ ), $t_{R}$ (minor enantiomer) $=26.424 \mathrm{~min}, t_{R}$ (major enantiomer) $=68.562 \mathrm{~min}, 99 \%$ ee . 


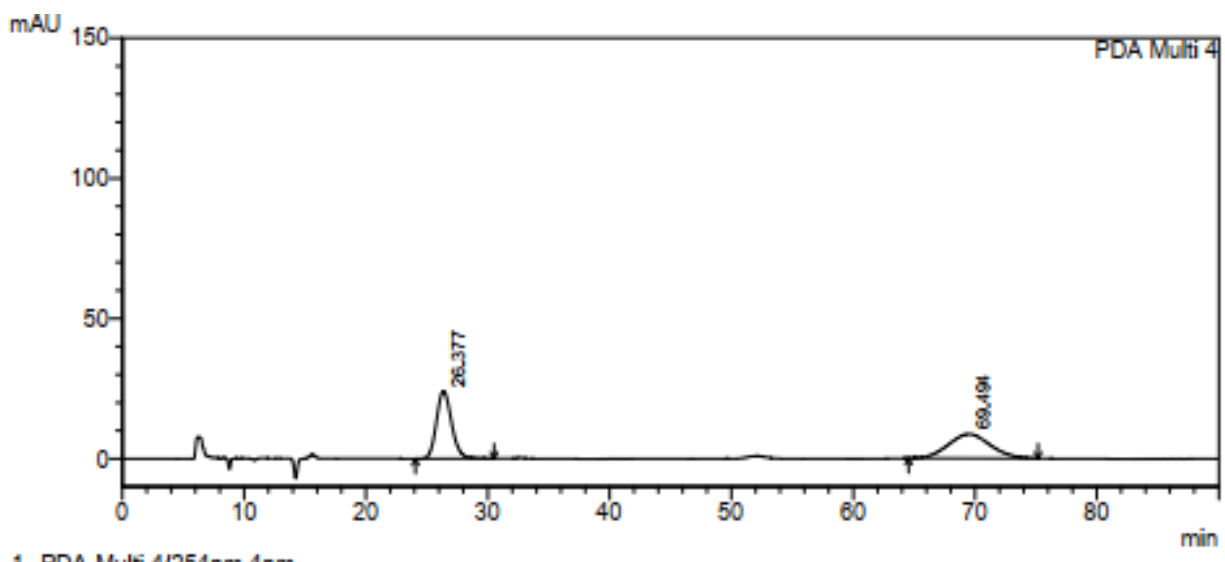

1 PDA Multi $4 / 254 \mathrm{~nm} 4 \mathrm{~nm}$
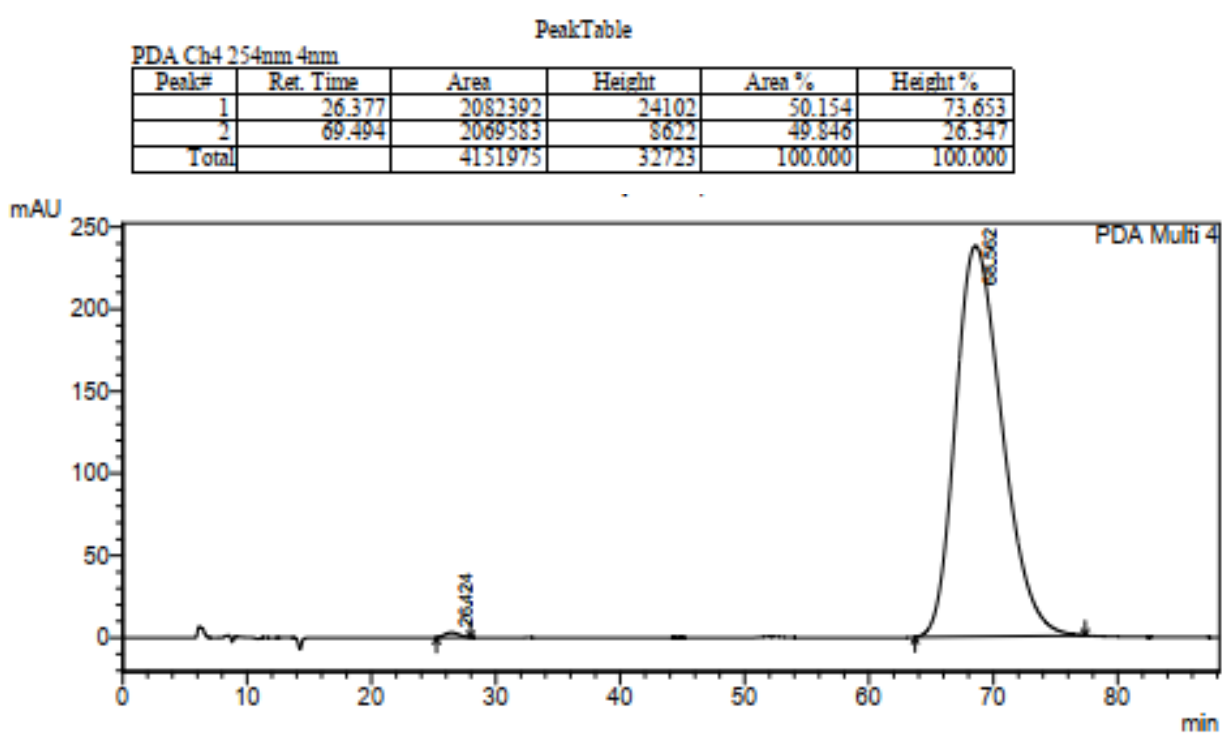

1 PDA Multi 4/254nm 4nm

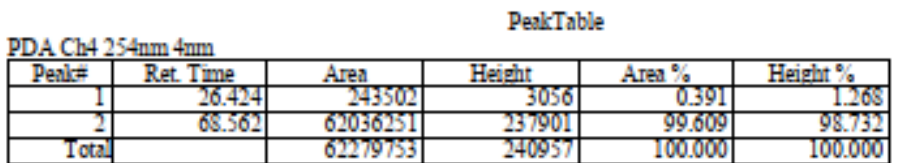

(S)-2-(1-benzyl-2-oxo-3-(thiophene-2-carboxamido)indolin-3-yl)ethane-1-sulfonyl fluoride (3t)<smiles>O=C(N[C@]1(CCS(=O)(=O)F)C(=O)N(Cc2ccccc2)c2ccccc21)c1cccs1</smiles>

Petroleum ether / ethyl acetate $=8: 1$ was used as the eluent for column chromatography. White solid (44.2 mg, 97\%), m. p. 235.5-236.6 ${ }^{\circ} \mathrm{C} .{ }^{1} \mathbf{H}$ NMR $\left(400 \mathrm{MHz}, \mathrm{CDCl}_{3}\right) \delta 7.55$ (d, $\left.J=3.7 \mathrm{~Hz}, 1 \mathrm{H}\right), 7.47$ (d, $J=4.9 \mathrm{~Hz}, 1 \mathrm{H}), 7.42-7.34$ (m, 5H), $7.29(\mathrm{t}, J=6.9 \mathrm{~Hz}, 1 \mathrm{H}), 7.25-7.19$ (m, 2H), 7.10-7.02 (m, 2H), 6.80 (d, $J=7.9 \mathrm{~Hz}, 1 \mathrm{H}$ ), 5.00 (dd, $J=43.0,15.7 \mathrm{~Hz}, 2 \mathrm{H}$ ), 3.73 (qd, $J=10.6,5.5 \mathrm{~Hz}, 1 \mathrm{H}$ ), 3.44 (ddd, $J=14.8$, 9.1, $5.0 \mathrm{~Hz}, 1 \mathrm{H}$ ), 2.82 (ddd, $J=14.8,9.8,5.3 \mathrm{~Hz}, 1 \mathrm{H}$ ), 2.56-2.44 (m, 1H). ${ }^{13} \mathrm{C}$ NMR (101 MHz, $\mathrm{CDCl}_{3}$ ) 
$\delta 175.07,161.40,142.10,137.06,135.24,131.36,129.85,129.35,129.01,128.20,127.91,127.86$, 127.26, 123.67, 123.09, 110.05, 59.97, 44.95 (d, $\left.J_{\mathrm{C}-\mathrm{F}}=18.2 \mathrm{~Hz}\right), 44.43,30.40 .{ }^{19} \mathbf{F}$ NMR (376 MHz, $\left.\mathrm{CDCl}_{3}\right) \delta$ 53.32. HRMS (m/z): Calcd for $\mathrm{C}_{22} \mathrm{H}_{19} \mathrm{~N}_{2} \mathrm{O}_{4} \mathrm{FS}_{2}$, $[\mathrm{M}+\mathrm{Na}]^{+}$, 481.0662; found 481.0677. $[\alpha]_{\mathrm{D}}^{20}=$ +23.7 (c 1.0, $\mathrm{CH}_{2} \mathrm{Cl}_{2}$ ). Chiral HPLC analysis (Chiral pak AS-H, $i-\mathrm{PrOH} / n$-hexane $=20: 80$, flow rate = $0.5 \mathrm{~mL} / \mathrm{min}$, wave length=254 nm), $\mathrm{t}_{\mathrm{R}}$ (minor enantiomer) $=18.235 \mathrm{~min}, \mathrm{t}_{\mathrm{R}}$ (major enantiomer) $=$ 29.124min, 99\% ee.

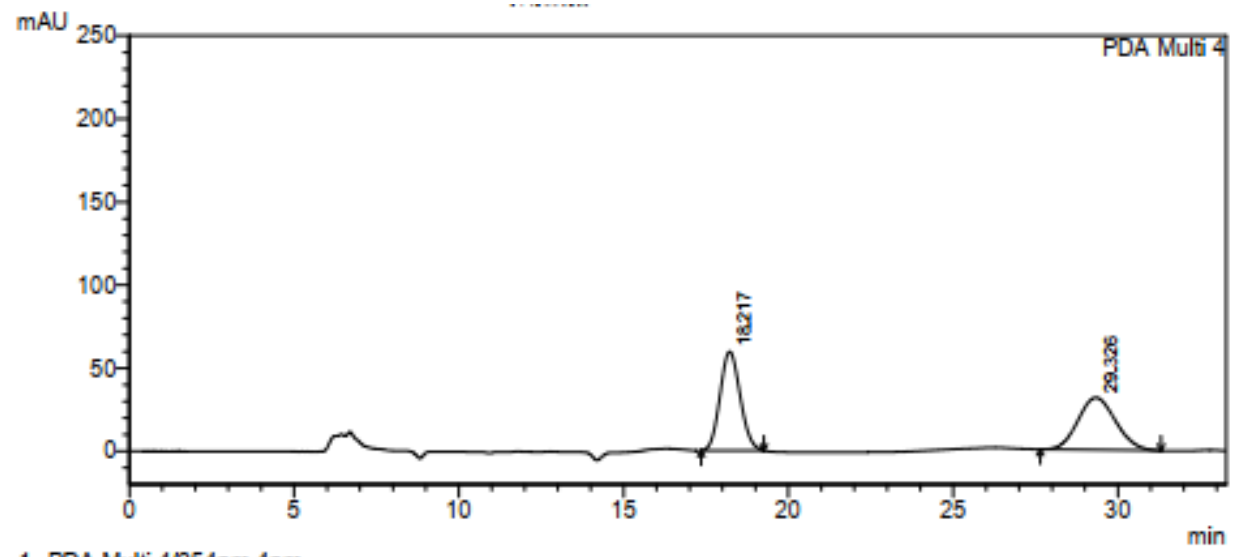

1 PDA Multi 4/254nm 4nm
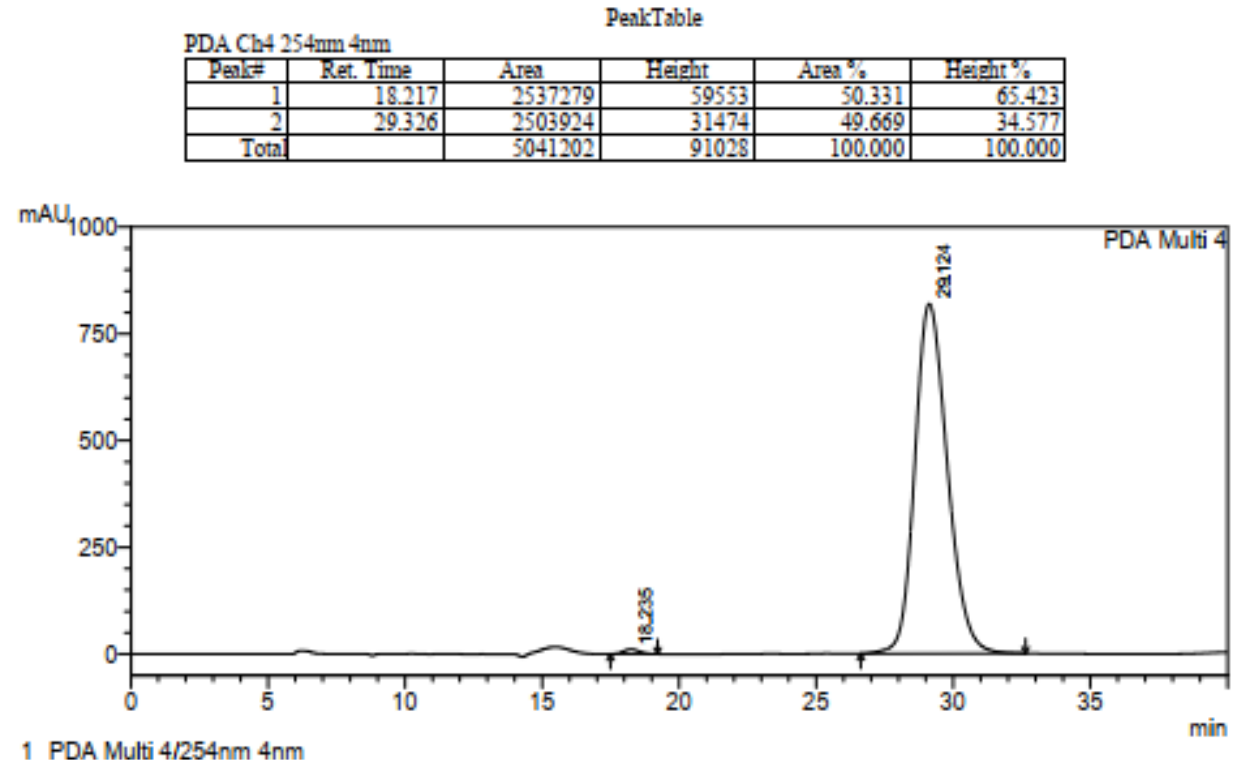

1 PDA Multi $4 / 254 \mathrm{~nm} 4 \mathrm{~nm}$

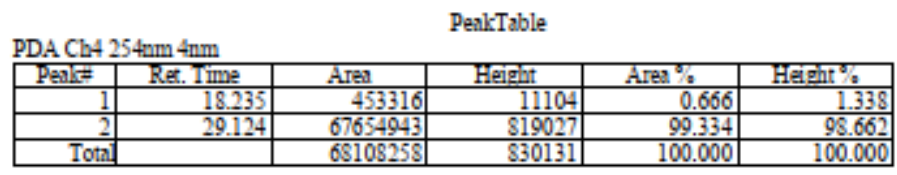

(S)-2-(1-benzyl-3-(furan-2-carboxamido)-2-oxoindolin-3-yl)ethane-1-sulfonyl fluoride (3u) 
<smiles>O=C(NC[C@@]1(CCS(=O)(=O)F)C(=O)N(Cc2ccccc2)c2ccccc21)c1ccco1</smiles>

Petroleum ether / ethyl acetate $=8: 1$ was used as the eluent for column chromatography. White solid (41 mg, 93\%), m. p. 211.7-212.9 ${ }^{\circ} \mathrm{C} .{ }^{1} \mathbf{H}$ NMR (400 MHz, $\mathrm{CDCl}_{3}$ ) $\delta$ 7.54-7.43 (m, 2H), 7.43-7.27 (m, 5H), 7.23 (dd, $J=8.0,1.2 \mathrm{~Hz}, 1 \mathrm{H}$ ), 7.14-6.97 (m, 3H), 6.79 (d, $J=8.0 \mathrm{~Hz}, 1 \mathrm{H}$ ), 6.51 (dd, $J=3.6,1.6$ Hz, 1H), 5.00 (dd, $J=26.8,16.0$ Hz, 2H), 3.63-3.44 (m, 2H), 2.91 (ddd, $J=13.610 .4,5.2 \mathrm{~Hz}, 1 \mathrm{H}$ ), 2.48 (ddd, $J=14.0,11.2,4.8 \mathrm{~Hz}, 1 \mathrm{H}) .{ }^{13} \mathrm{C}$ NMR $\left(101 \mathrm{MHz}, \mathrm{CDCl}_{3}\right) \delta 173.42,156.53,145.56,143.60,141.16$, 134.17, 128.93, 127.98, 126.89, 126.27, 122.64, 122.38, 114.76, 111.43, 108.97, 58.49, 43.97 (d, $J_{\mathrm{C}-\mathrm{F}}=$ 18.4 Hz), 43.40, 29.47. ${ }^{19} \mathbf{F}$ NMR (376 MHz, $\left.\mathrm{CDCl}_{3}\right) \delta$ 53.39. HRMS ESI (m/z): Calcd for

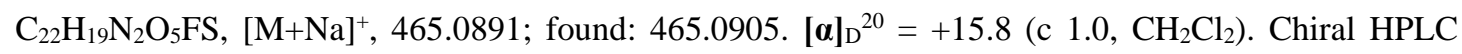
analysis (Chiral pak AS-H, $i$-PrOH$/ n$-hexane $=20: 80$, flow rate $=0.5 \mathrm{~mL} / \mathrm{min}$, wave length $=254 \mathrm{~nm}$ ), $t_{\mathrm{R}}($ minor enantiomer $)=19.373 \mathrm{~min}, \mathrm{t}_{\mathrm{R}}($ major enantiomer $)=28.519 \mathrm{~min}, 97 \%$ ee.

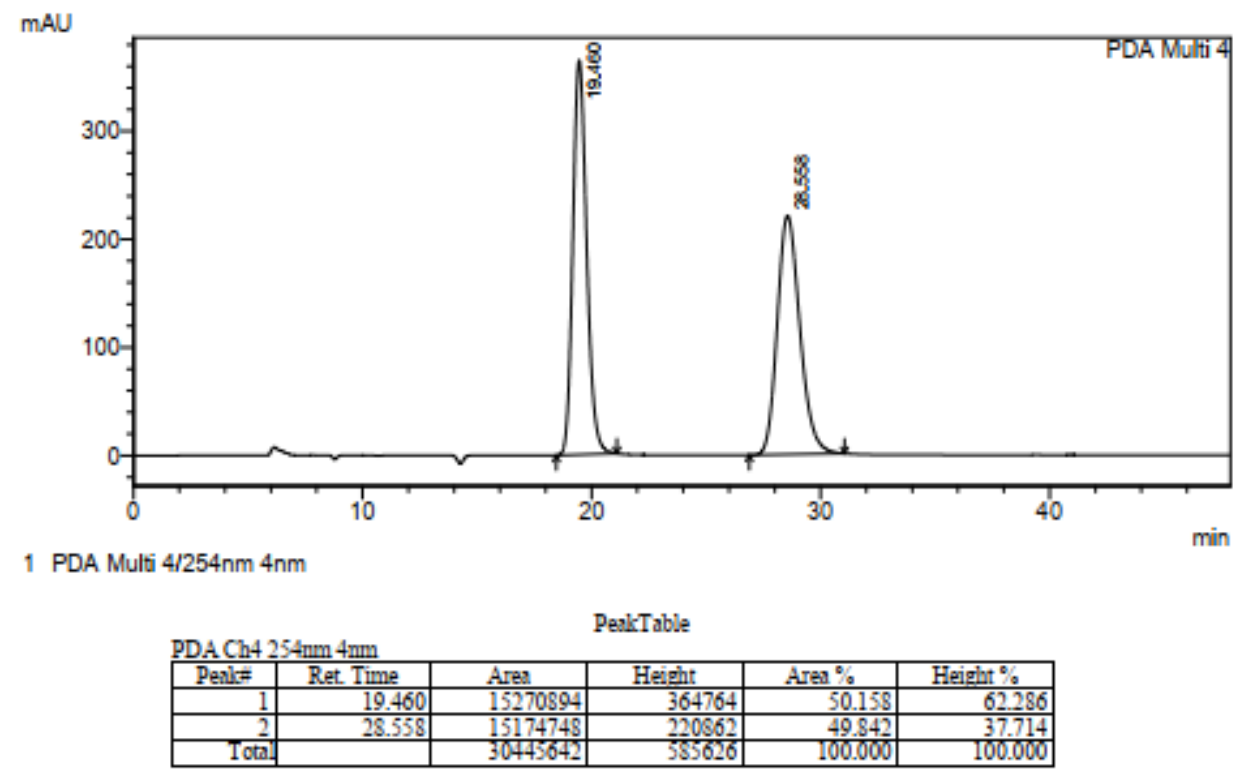



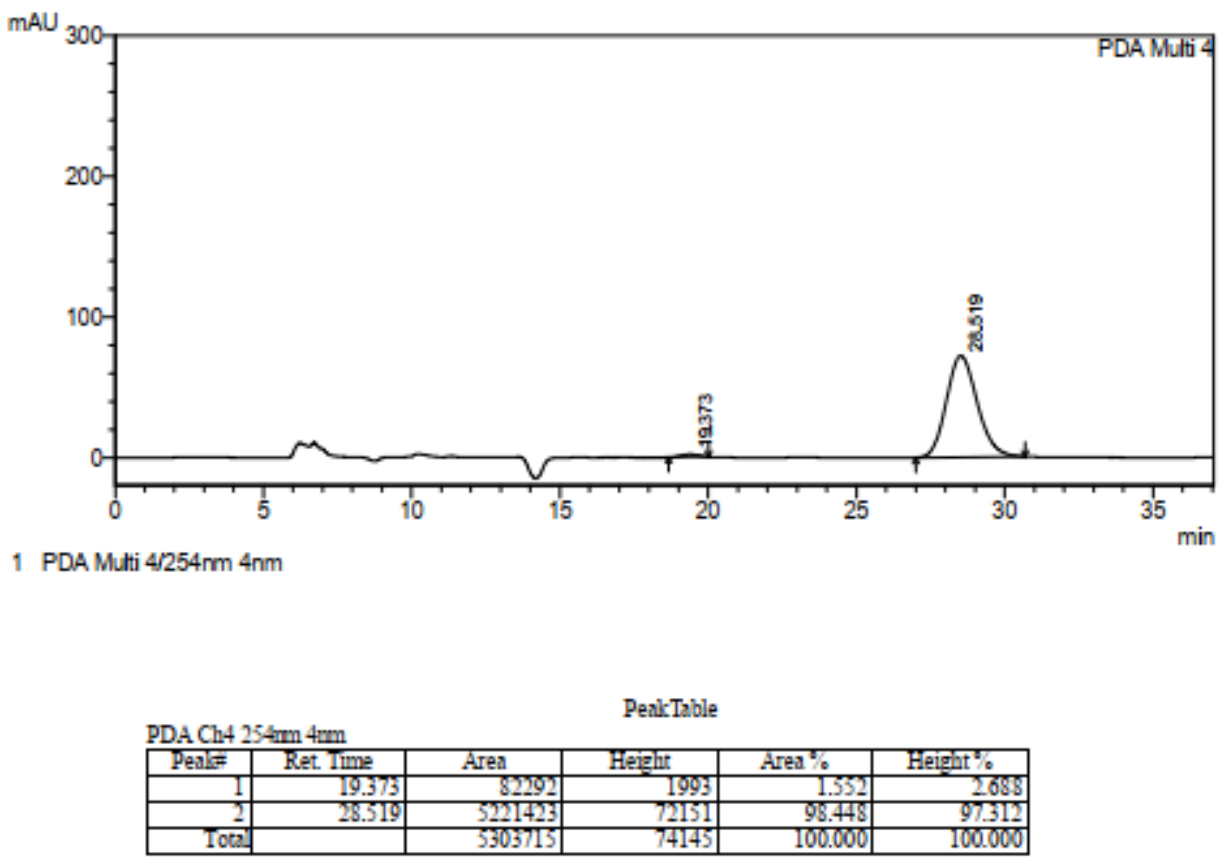

(S)-2-(1-benzyl-3-(cyclopropanecarboxamido)-2-oxoindolin-3-yl)ethane-1-sulfonyl fluoride (3v)<smiles>O=C(N[C@@]1(CCS(=O)(=O)F)C(=O)N(Cc2ccccc2)c2ccccc21)C1CC1</smiles>

Petroleum ether / ethyl acetate $=6: 1$ was used as the eluent for column chromatography. White solid (35 mg, 84\%), m. p. 211.9-213 C. ${ }^{1} \mathbf{H}$ NMR (400 MHz, $\left.\mathrm{CDCl}_{3}\right) \delta 7.39$ (d, J = 7.6 Hz, 1H), 7.37-7.26 (m, 5H), 7.22 (td, $J=8.0,1.2 \mathrm{~Hz}, 1 \mathrm{H}), 7.13-7.01$ (m, 1H), 6.77 (d, $J=8.0 \mathrm{~Hz}, 1 \mathrm{H}), 6.56$ (s, 1H), 4.96 (dd, $J$ $=57.2,15.6 \mathrm{~Hz}, 2 \mathrm{H}), 3.60-3.49(\mathrm{~m}, 1 \mathrm{H}), 3.48-3.38(\mathrm{~m}, 1 \mathrm{H}), 2.77$ (ddd, $J=14.0,10.8,4.8 \mathrm{~Hz}, 1 \mathrm{H}), 2.38$ (ddd, $J=14.0,11.2,5.2 \mathrm{~Hz}, 1 \mathrm{H}), 1.51-1.39$ (m, 1H), 1.02-0.92 (m, 1H), 0.91-0.84 (m, 1H), 0.82-0.70 (m, 2H). ${ }^{13} \mathrm{C}$ NMR (101 MHz, $\left.\mathrm{CDCl}_{3}\right) \delta 174.86,173.32$, 142.03, 135.30, 129.77, 128.99, 128.45, 127.92, 127.28, 123.63, 123.34, 109.90, 59.74, 45.06 (d, $\left.J_{\mathrm{C}-\mathrm{F}}=18.3 \mathrm{~Hz}\right), 44.36,30.37,14.36,7.96 .{ }^{19} \mathbf{F}$ NMR $\left(376 \mathrm{MHz}, \mathrm{CDCl}_{3}\right) \delta$ 53.26. HRMS ESI (m/z): Calcd for $\mathrm{C}_{21} \mathrm{H}_{21} \mathrm{~N}_{2} \mathrm{O}_{4} \mathrm{FS}$, [M+Na] $]^{+}$, 439.1098; found: 439.1110. $[\boldsymbol{\alpha}]_{\mathrm{D}}{ }^{20}=-23.9$ (c 1.0, $\mathrm{CH}_{2} \mathrm{Cl}_{2}$ ). Chiral HPLC analysis (Chiral pak AS-H, $i-\mathrm{PrOH} / n$-hexane = $20: 80$, flow rate $=0.5 \mathrm{~mL} / \mathrm{min}$, wave length $=254 \mathrm{~nm}), \mathrm{t}_{\mathrm{R}}($ minor enantiomer $)=19.882 \mathrm{~min}, \mathrm{t}_{\mathrm{R}}($ major enantiomer) $24.210 \mathrm{~min}, 92 \%$ ee. 


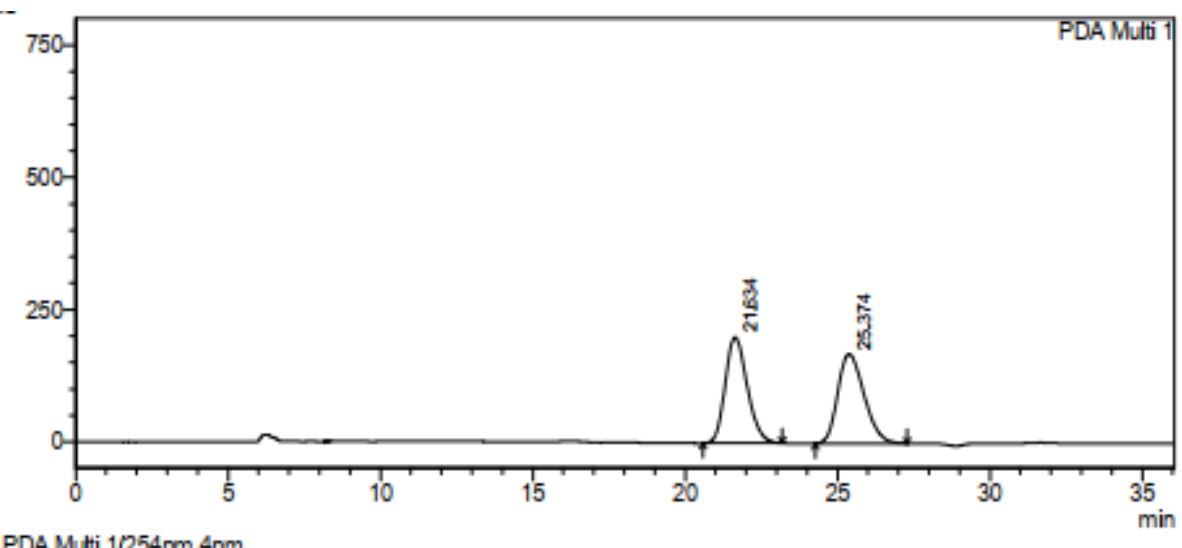

PDA Mutti $1 / 254 \mathrm{~mm} 4 \mathrm{~nm}$

PDAChl 254m 4mm

PenkTable

\begin{tabular}{|c|c|c|c|c|c|}
\hline PealF & Ret. Iime & Aren & Height & Area $\%$ & Heighr \% \\
\hline 1 & 21.634 & 10099703 & 200709 & 49.919 & 54.324 \\
\hline 2 & 25.374 & 10132467 & 168755 & 50.081 & 45.676 \\
\hline Tota. & & 20232170 & 369464 & 100000 & 100.000 \\
\hline
\end{tabular}

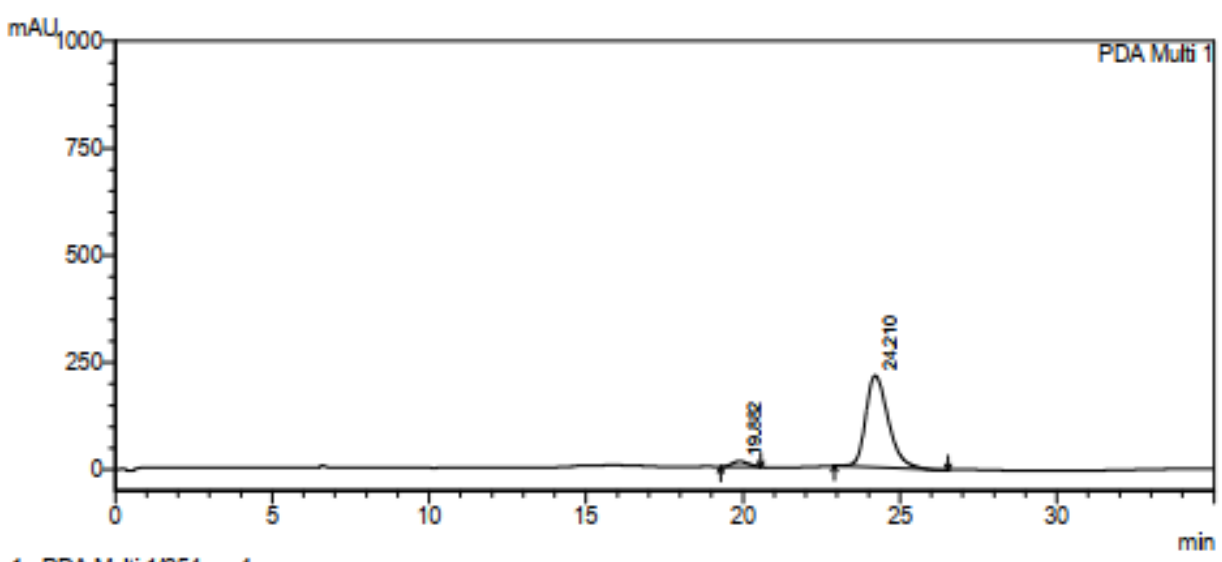

PDA Muti $1 / 254 \mathrm{~mm} 4 \mathrm{~nm}$

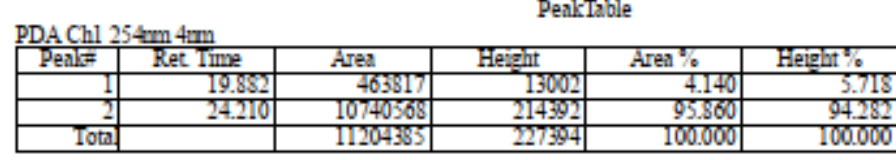

(S)-2-(1-benzyl-3-(cyclopentanecarboxamido)-2-oxoindolin-3-yl)ethane-1-sulfonyl fluoride (3w)<smiles>O=C(N[C@]1(CCS(=O)(=O)F)C(=O)N(Cc2ccccc2)c2ccccc21)C1CCCC1</smiles>

Petroleum ether / ethyl acetate $=6: 1$ was used as the eluent for column chromatography. White solid (33 mg, 75\%), m. p. 163.9-164.4 ${ }^{\circ} \mathrm{C} .{ }^{1} \mathbf{H}$ NMR (500 MHz, $\mathrm{CDCl}_{3}$ ) $\delta$ 7.38-7.32 (m, 5H), 7.30-7.26 (m, 1H), 7.22 (td, $J=8.0,1.5 \mathrm{~Hz}, 1 \mathrm{H}$ ), 7.06 (td, $J=7.5,0.5 \mathrm{~Hz}, 1 \mathrm{H}$ ), 6.77 (d, $J=8.0 \mathrm{~Hz}, 1 \mathrm{H}$ ), 6.38 (s, $1 \mathrm{H}$ ), 4.96 (dd, $J=81.5,17.0 \mathrm{~Hz}, 2 \mathrm{H}$ ), 3.60-3.47 (m, 1H), 3.45-3.34 (m, 1H), 2.73 (ddd, $J=14.0,10.5,5.0 \mathrm{~Hz}$, 1H), 2.65-2.57 (m, 1H), 2.35 (ddd, $J=14.0,10.5,5.0 \mathrm{~Hz}, 1 \mathrm{H}), 1.88-1.81$ (m, 2H), 1.80-1.74 (m, 1H), 1.70-1.65 (m, 3H), 1.59-1.51 (m, 2H). ${ }^{13}$ C NMR (126 MHz, $\left.\mathrm{CDCl}_{3}\right) \delta$ 175.99, 174.90, 142.14, 135.38, 129.74, 129.00, 128.50, 127.91, 127.31, 123.59, 122.97, 109.91, 59.50, 45.00 (d, $\left.J_{\mathrm{C}-\mathrm{F}}=18.2 \mathrm{~Hz}\right), 44.83$, 
44.38, 30.48, 30.33, 29.93, 26.03, 25.96. ${ }^{19}$ F NMR (471 MHz, $\left.\mathrm{CDCl}_{3}\right) \delta$ 53.26. HRMS ESI (m/z): Calcd

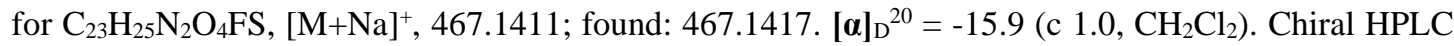
analysis (Chiral pak AS-H, $i$-PrOH $/ n$-hexane $=20: 80$, flow rate $=0.5 \mathrm{~mL} / \mathrm{min}$, wave length $=254 \mathrm{~nm}$ ), $t_{R}($ minor enantiomer $)=29.540 \mathrm{~min}, t_{R}$ (major enantiomer $)=37.492 \mathrm{~min}$, 95\% ee. mAL

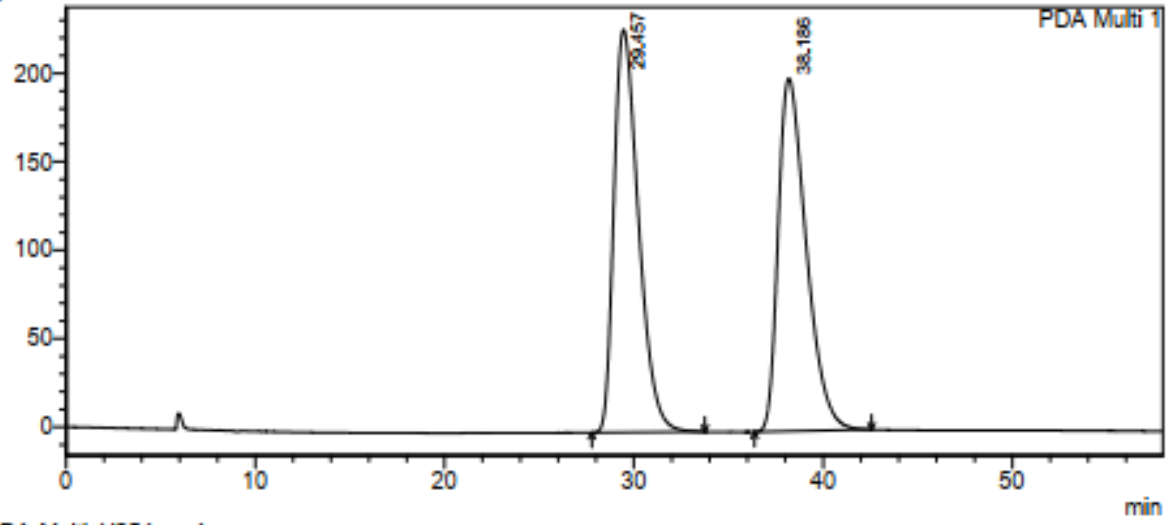

1 PDA Multi $1 / 254 \mathrm{~nm} 4 \mathrm{~nm}$

Penk:Table

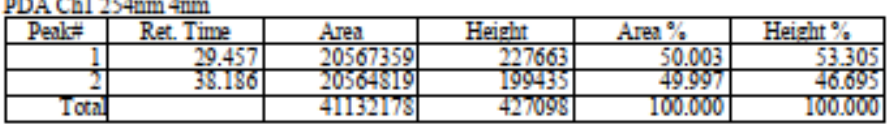

mAl

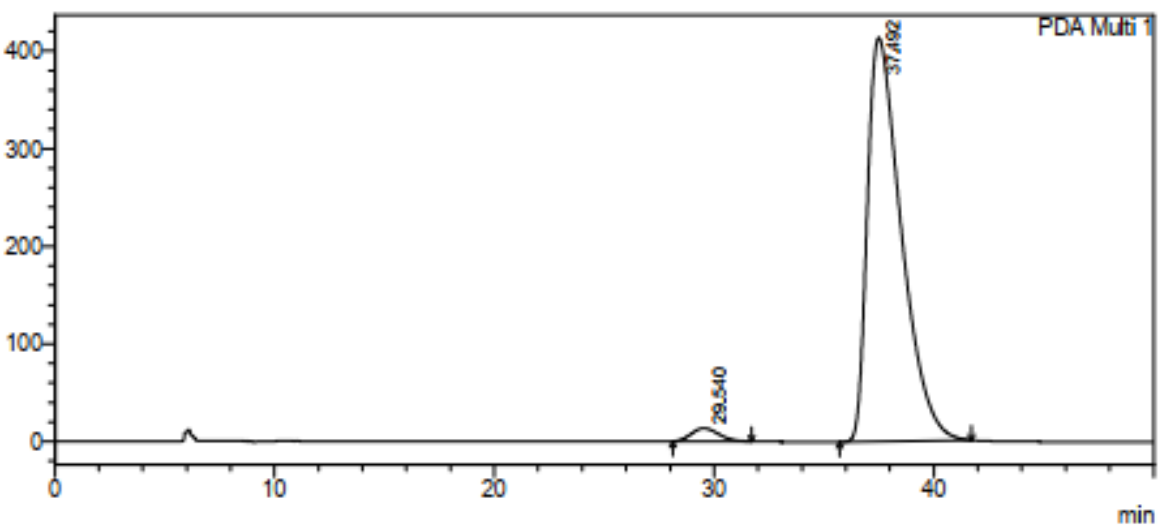

1 PDA Mutti 1/254nm 4nm

PeakTable

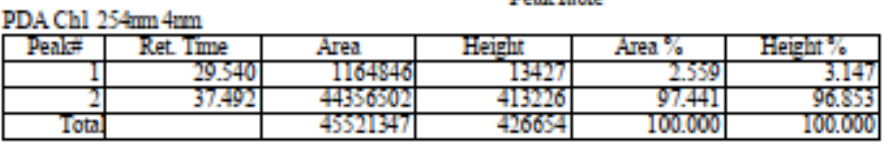

(S)-2-(1-benzyl-2-oxo-3-(tetrahydro-2H-pyran-4-carboxamido)indolin-3-yl)ethane-1-sulfonyl fluoride (3x)<smiles>O=C(N[C@@]1(CCS(=O)(=O)F)C(=O)N(Cc2ccccc2)c2ccccc21)C1CCOCC1</smiles> 
Petroleum ether / ethyl acetate $=6: 1$ was used as the eluent for column chromatography. White solid (22 mg, 46\%), m. p. 239.8-240.9 ${ }^{\circ} \mathrm{C} .{ }^{1} \mathbf{H}$ NMR (400 MHz, $\left.\mathrm{CDCl}_{3}\right) \delta$ 7.38-7.32 (m, 5H), 7.31-7.27 (m, 1H), 7.23 (td, $J=8.0,1.5 \mathrm{~Hz}, 1 \mathrm{H}), 7.07$ (td, $J=7.5,0.5 \mathrm{~Hz}, 1 \mathrm{H}), 6.78$ (d, $J=8.0 \mathrm{~Hz}, 1 \mathrm{H}), 6.47$ (s, $1 \mathrm{H})$, 4.96 (dd, $J=47.0,15.5$ Hz, 2H), 4.02-3.95 (m, 2H), 3.63-3.55 (m, 1H), 3.43-3.34 (m, 3H), 2.72 (ddd, $J$ $=14.5,10.0,5.0 \mathrm{~Hz}, 1 \mathrm{H}), 2.44-2.33(\mathrm{~m}, 2 \mathrm{H}), 1.82-1.69(\mathrm{~m}, 5 \mathrm{H}) .{ }^{13} \mathrm{C}$ NMR $\left(126 \mathrm{MHz}, \mathrm{CDCl}_{3}\right) \delta 174.72$, 173.95, 142.13, 135.25, 129.88, 129.01, 128.27, 127.95, 127.30, 123.65, 122.88, 110.00, 67.05, 67.02, 59.39, 44.91 (d, $\left.J_{\mathrm{C}-\mathrm{F}}=18.2 \mathrm{~Hz}\right), 44.40,41.46,30.28,29.10,28.80 .{ }^{19} \mathbf{F}$ NMR $\left(471 \mathrm{MHz}, \mathrm{CDCl}_{3}\right) \delta 53.28$.

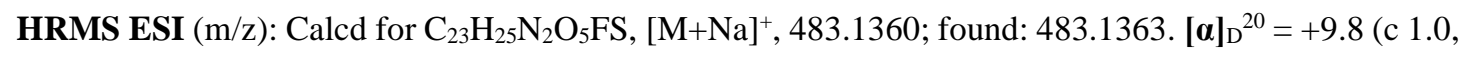
$\mathrm{CH}_{2} \mathrm{Cl}_{2}$ ). Chiral HPLC analysis (Chiral pak AS-H, $i$-PrOH$/ n$-hexane $=20: 80$, flow rate $=0.5 \mathrm{~mL} / \mathrm{min}$, wave length $=254 \mathrm{~nm}), \mathrm{t}_{\mathrm{R}}($ minor enantiomer $)=23.256 \mathrm{~min}, \mathrm{t}_{\mathrm{R}}($ major enantiomer $)=28.497 \mathrm{~min}, 94 \%$ ee.

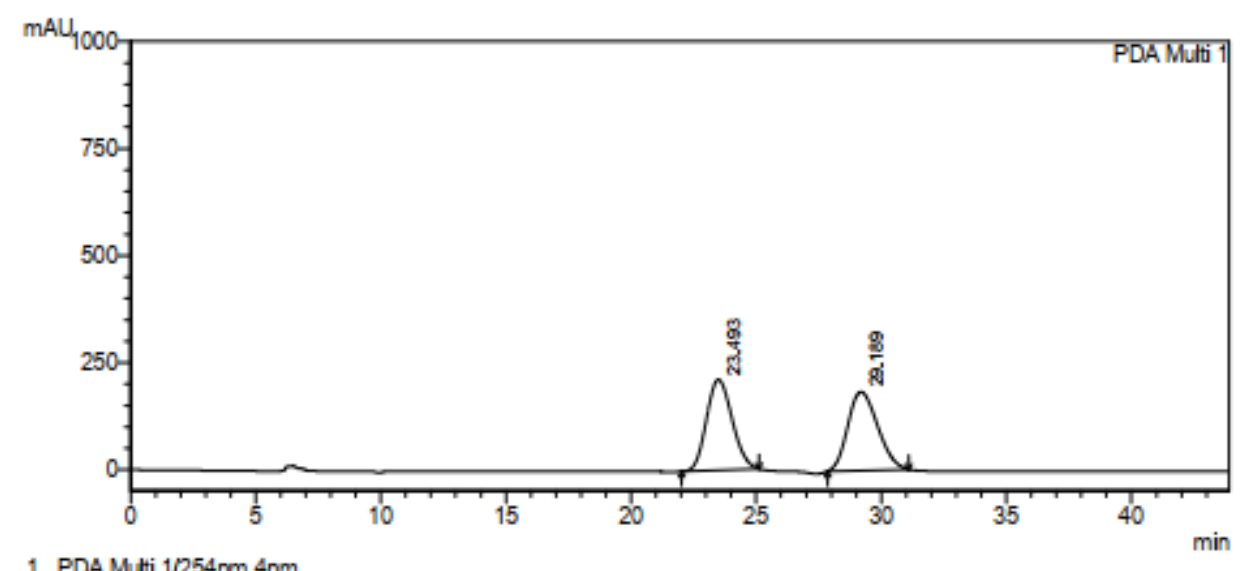

1 PDA Muti $1 / 254 \mathrm{~mm} 4 \mathrm{~nm}$
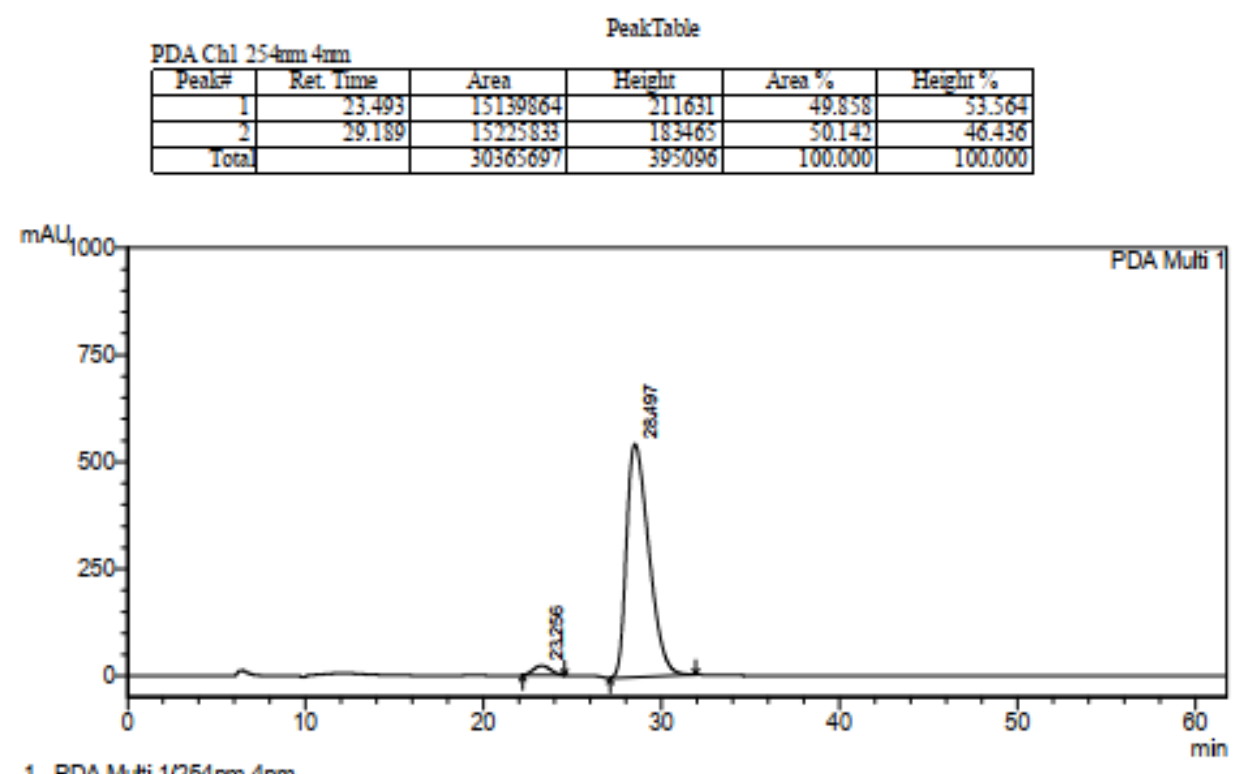

1 PDA Mutti $1 / 254 \mathrm{~mm} 4 \mathrm{~nm}$

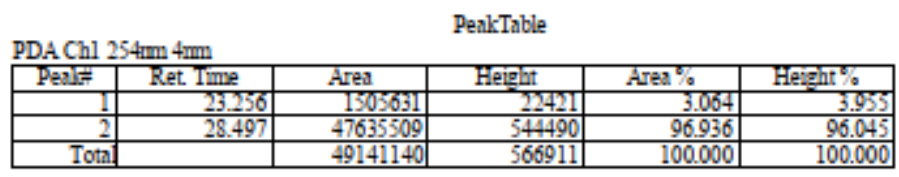

ethyl (S)-(1-benzyl-3-(2-(fluorosulfonyl)ethyl)-2-oxoindolin-3-yl)carbamate (3y) 
<smiles>CCOS(=O)(=O)CC[C@@]1(NC(=O)OF)C(=O)N(Cc2ccccc2)c2ccccc21</smiles>

3y

Petroleum ether / ethyl acetate $=8: 1$ was used as the eluent for column chromatography. White solid (29 mg, 70\%), m. p. $207.9-208.7^{\circ} \mathrm{C} .{ }^{1} \mathbf{H}$ NMR(400 MHz, $\left.\mathrm{CDCl}_{3}\right) \delta 7.41$ (d, $J=7.5 \mathrm{~Hz}, 1 \mathrm{H}$ ), 7.37-7.28 (m, 5H), 7.28-7.23 (m, 2H), 7.12-7.07 (m, 1H), 6.79 (d, $J=7.5$ Hz, 1H), 5.67 (s, 1H), 4.99-4.90 (m, 2H), 4.09-3.96 (m, 2H), 3.55-3.47 (m, 2H), 2.76-2.67 (m, 1H), 2.39-2.31 (m, 1H), 1.12 (s, 3H). ${ }^{13} \mathbf{C}$ NMR $\left(101 \mathrm{MHz}, \mathrm{CDCl}_{3}\right) \delta 174.86,154.85,142.01,135.18,129.92,128.97,127.97,127.36,123.63,123.36$, 109.90, 61.75, 59.62, 45.07 (d, $\left.J_{\mathrm{C}-\mathrm{F}}=18.5 \mathrm{~Hz}\right), 44.33,30.62,14.26 .{ }^{19} \mathbf{F}$ NMR $\left(471 \mathrm{MHz}, \mathrm{CDCl}_{3}\right) \delta 53.32$. HRMS ESI (m/z): Calcd for $\mathrm{C}_{20} \mathrm{H}_{21} \mathrm{~N}_{2} \mathrm{O}_{5} \mathrm{FS}$, [M+Na $]^{+}$, 443.1047; found: 443.1049. [ $\left.\alpha\right]_{\mathrm{D}}{ }^{20}=-16.9$ (c 1.0, $\mathrm{CH}_{2} \mathrm{Cl}_{2}$ ). Chiral HPLC analysis (Chiral pak AS-H, $i-\mathrm{PrOH} / n$-hexane $=20: 80$, flow rate $=0.5 \mathrm{~mL} / \mathrm{min}$, wave length $=254 \mathrm{~nm}), \mathrm{t}_{\mathrm{R}}($ minor enantiomer $)=12.691 \mathrm{~min}, \mathrm{t}_{\mathrm{R}}($ major enantiomer $)=15.989 \mathrm{~min}, 90 \%$ ee.

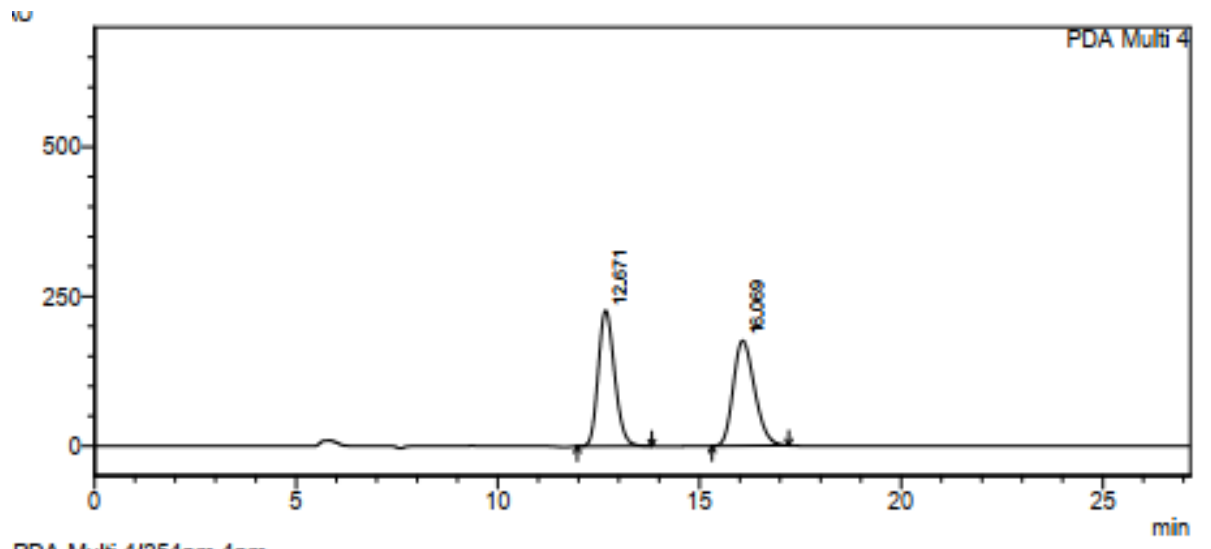

PDA Multi 4/254nm 4nm

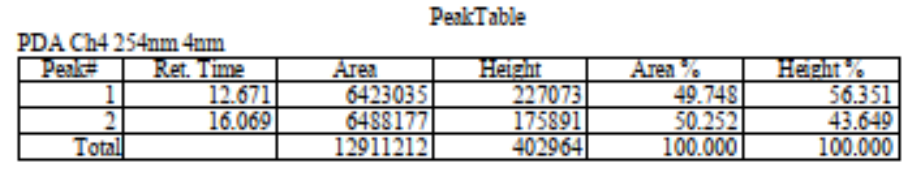

mAU

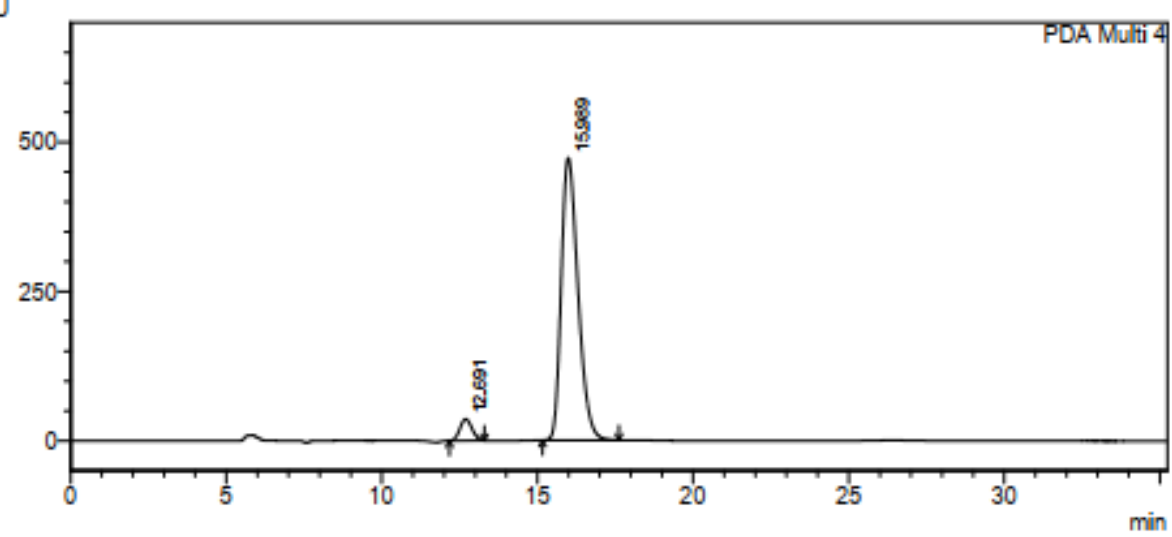

1 PDA Multi 4/254nm 4nm

Penk:Table

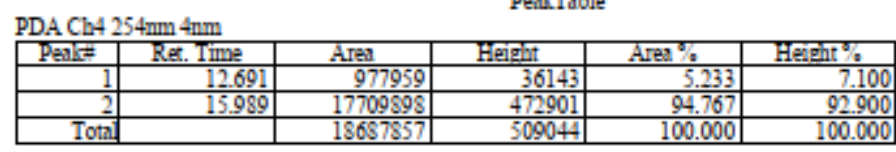




\section{Typical procedures and characterization data for compounds 5}

\subsection{General procedure for the synthesis of compounds 5}<smiles>[R]C(=O)N[C@]1(CCS(=O)(=O)F)C(=O)Nc2cc[R]#cc21</smiles>

$\mathrm{R}^{1}$

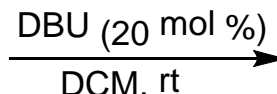

$\mathrm{DCM}, \mathrm{rt} \longrightarrow$

$3 a, 3 m, 3 r$<smiles>[R]C[R]1ccc2c(c1)[C@@]1(CCS(=O)(=O)N(C([R])=O)C1)C(=O)N2[R1]</smiles>

$5 a, 5 m, 5 r$

To a stirred solution of $3(0.1 \mathrm{mmol})$ in $\mathrm{CH}_{2} \mathrm{Cl}_{2}(1.0 \mathrm{~mL})$ was added $\mathrm{DBU}$ (3 $\left.\mathrm{mg}, 0.02 \mathrm{mmol}\right)$. The mixture was stirred at rt for $12 \mathrm{~h}$. After the completion of the reaction (monitored by TLC), the mixture was concentrated under vacuum. The residue was purified by flash chromatography over silica gel to afford the products 5 .

4.2 General procedure for the synthesis of racemic products 5<smiles>[R]C(=O)N[C@H]1C(=O)N([R])c2cc([R])ccc21</smiles>

1a, 1m, 1r

$2 a$

$5 a, 5 m, 5 r$

A solution of 3-benzamido-2-oxindole 1 (0.1 mmol), ethylene sulfonyl fluoride 2a (13.2 mg, $0.12 \mathrm{mmol}$ ), $\mathrm{NaHCO}_{3}$ (16.8 mg, $0.2 \mathrm{mmol}$ ) and $\mathrm{DBU}$ (3 mg, $\left.0.02 \mathrm{mmol}\right)$ in $\mathrm{CH}_{2} \mathrm{Cl}_{2}(1.0 \mathrm{~mL})$ was stirred at rt for 12 h. After the solvent was evaporated under vacuum, the residue was purified by flash column chromatography over silica gel (petroleum ether/EtOAc $=8: 1$ ) to afford the racemic products 5.

\subsection{Scale-up synthesis of compound 5a}<smiles>O=C(N[C@]1(CCS(=O)(=O)F)C(=O)N(Cc2ccccc2)c2ccccc21)c1ccccc1</smiles>

To a stirred solution of 3a (678 mg, $1.5 \mathrm{mmol})$ in $\mathrm{CH}_{2} \mathrm{Cl}_{2}(15 \mathrm{~mL})$ was added DBU (46 mg, $0.3 \mathrm{mmol}$ ). The mixture was stirred at rt for $12 \mathrm{~h}$. After the completion of the reaction (monitored by TLC), the mixture was concentrated under vacuum. The residue was purified by flash chromatography over silica gel (petroleum ether / ethyl acetate $=8: 1$ ) to afford the product 5a as a white solid (637 $\mathrm{mg}, 98 \%$ ).

\subsection{Characterizations of products 5}

(S)-2'-benzoyl-1-benzylspiro[indoline-3,3'-isothiazolidin]-2-one 1',1'-dioxide (5a) 
<smiles>O=C1Nc2ccccc2C1C1CCS(=O)(=O)N1C(=O)c1ccccc1</smiles>

5 a $\mathrm{Bn}$

Petroleum ether / ethyl acetate $=8: 1$ was used as the eluent for column chromatography. White solid (44.6 mg, 99\%), m.p. $194.5-195.9^{\circ} \mathrm{C} .{ }^{1} \mathbf{H}$ NMR (500 MHz, $\left.\mathrm{CDCl}_{3}\right) \delta 7.85$ (d, $J=7.5 \mathrm{~Hz}, 2 \mathrm{H}$ ), 7.59-7.52 (m, 1H), 7.50-7.40 (m, 5H), 7.36 (t, $J=7.5 \mathrm{~Hz}, 2 \mathrm{H}$ ), 7.32-7.26 (m, 1H), 7.24-7.18 (m, 1H), 7.06 (t, $J=$ $7.5 \mathrm{~Hz}, 1 \mathrm{H}$ ), 6.72 (d, $J=8.0 \mathrm{~Hz}, 1 \mathrm{H}$ ), 5.05-4.92 (m, 2H), 4.11 (td, $J=13.0,6.5 \mathrm{~Hz}, 1 \mathrm{H}$ ), 3.47 (dd, $J=$ 12.0, $5.5 \mathrm{~Hz}, 1 \mathrm{H}$ ), 2.80 (td, $J=14.0,5.5 \mathrm{~Hz}, 1 \mathrm{H}), 2.62$ (dd, $J=13.5,6.0 \mathrm{~Hz}, 1 \mathrm{H}) .{ }^{13} \mathbf{C}$ NMR $(126 \mathrm{MHz}$, $\left.\mathrm{CDCl}_{3}\right) \delta 174.65,167.79,142.84,135.39,133.46,132.96,130.23,128.91,128.77,128.38,127.78$, 127.31, 126.96, 123.53, 122.29, 109.94, 65.68, 47.14, 44.35, 29.66. HRMS ESI (m/z): Calcd for $\mathrm{C}_{20} \mathrm{H}_{21} \mathrm{~N}_{2} \mathrm{O}_{5} \mathrm{FS}$, [M+Na] $]^{+}$, 443.1047; found: 443.1049. $[\alpha]_{\mathrm{D}}{ }^{20}=+21.3$ (c 1.0, $\mathrm{CH}_{2} \mathrm{Cl}_{2}$ ). Chiral HPLC analysis (Chiral pak OD-H, $i-\mathrm{PrOH} / n$-hexane $=20: 80$, flow rate $=1.0 \mathrm{~mL} / \mathrm{min}$, wave length $=254 \mathrm{~nm}$ ), $t_{R}($ major enantiomer $)=15.382 \mathrm{~min}, t_{R}($ minor enantiomer $)=18.639 \mathrm{~min}, 99 \%$ ee.

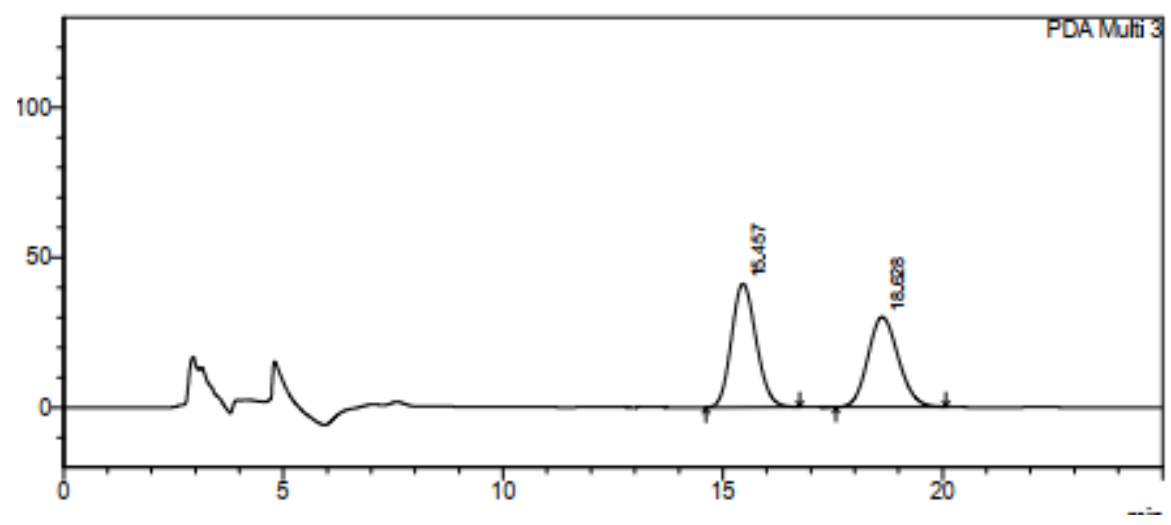

1 PDA Muti $3 / 254 \mathrm{~mm} 4 \mathrm{~nm}$

PeakTable
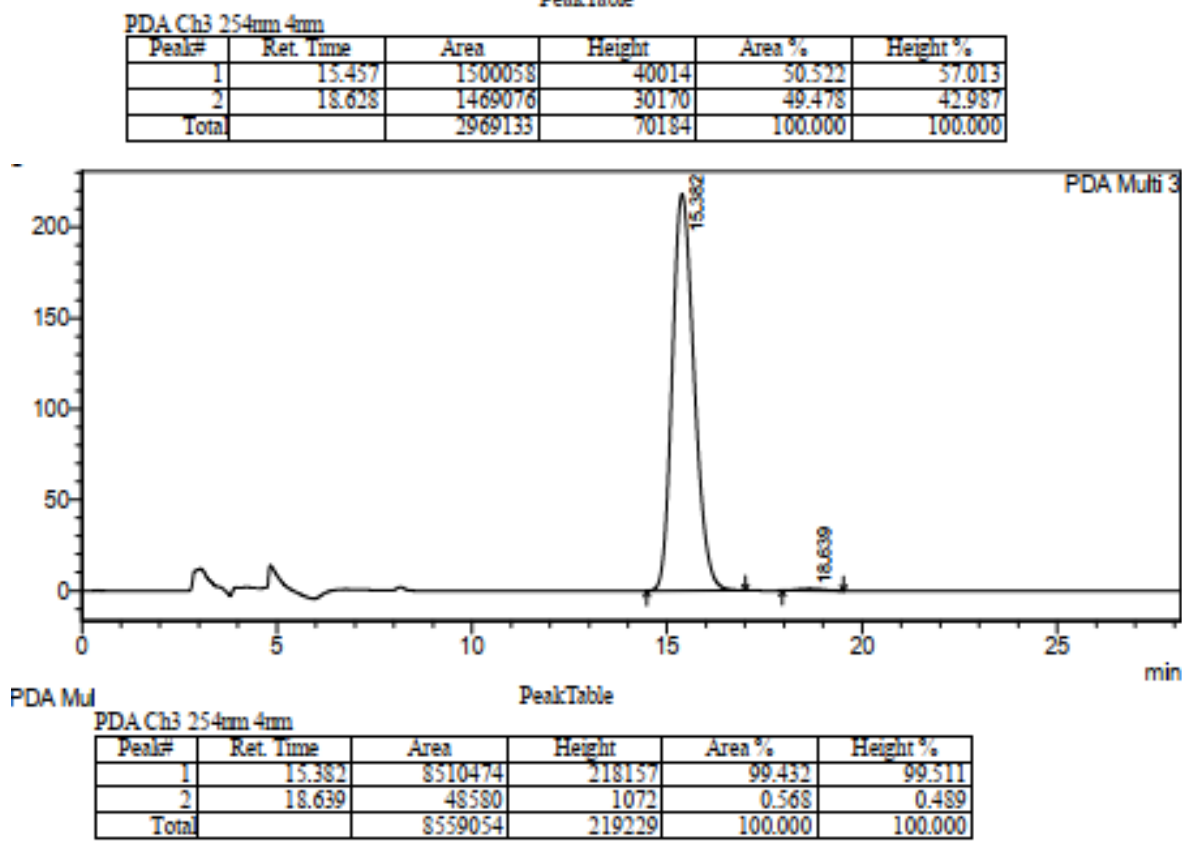

(S)-2'-benzoyl-1-benzyl-7-fluorospiro[indoline-3,3'-isothiazolidin]-2-one 1',1'-dioxide (5m) 
<smiles>O=C(c1ccccc1)N1C2(CCS1(=O)=O)C(=O)N(Cc1ccccc1)c1c(F)cccc12</smiles>

Petroleum ether / ethyl acetate $=8: 1$ was used as the eluent for column chromatography. White solid (46.5 mg, 99\%), m. p. $183.5-184.7^{\circ} \mathrm{C} .{ }^{1} \mathbf{H}$ NMR (500 MHz, CDCl $) \delta 7.85$ (d, J = 7.5 Hz, 2H), 7.60-7.54 (m, 1H), 7.49-7.43 (m, 4H), 7.38-7.32( m, 2H), 7.32-7.27 (m, 1H), 7.23 -7.18 (m, 1H), 7.05-6.98 (m, 2H), 5.20-5.07 (m, 2H), 4.16-4.03 ( m, 1H), 3.47 (dd, $J=12.0,5.5$ Hz, 1H), 2.76 (td, $J=14.0,5.5 \mathrm{~Hz}$, 1H), 2.59 (dd, $J=14.0,6.5 \mathrm{~Hz}, 1 \mathrm{H}) .{ }^{13} \mathrm{C}$ NMR $\left(126 \mathrm{MHz}, \mathrm{CDCl}_{3}\right) \delta 174.33,167.75,147.50\left(\mathrm{~d}, J_{\mathrm{C}-\mathrm{F}}=\right.$ $246.1 \mathrm{~Hz}), 136.67,133.18,133.08, \delta 129.89$ (d, JC-F $=3.5 \mathrm{~Hz}), 129.54$ (d, JC-F $=9.3 \mathrm{~Hz}), 128.73,128.66$, 128.42, 127.70, 127.48, 124.33 (d, JC-F $=6.5 \mathrm{~Hz}), 118.47$ (d, $\left.J_{\mathrm{C}-\mathrm{F}}=19.7 \mathrm{~Hz}\right), 118.12\left(\mathrm{~d}, J_{\mathrm{C}-\mathrm{F}}=3.2 \mathrm{~Hz}\right)$, 65.57, 47.02, 45.90 (d, JC-F $=4.5 \mathrm{~Hz}), 29.65$. HRMS ESI (m/z): Calcd for $\mathrm{C}_{20} \mathrm{H}_{21} \mathrm{~N}_{2} \mathrm{O}_{5} \mathrm{FS}$, $[\mathrm{M}+\mathrm{Na}]^{+}$, 443.1047; found: 443.1049. [a $]_{\mathrm{D}}{ }^{20}=+13.2\left(\mathrm{c} 1.0, \mathrm{CH}_{2} \mathrm{Cl}_{2}\right)$. Chiral HPLC analysis (Chiral pak AS-H, $i-$ $\mathrm{PrOH} / n$-hexane $=20: 80$, flow rate $=0.5 \mathrm{~mL} / \mathrm{min}$, wave length $=254 \mathrm{~nm}), \mathrm{t}_{\mathrm{R}}($ minor enantiomer $)=$ $15.367 \mathrm{~min}, \mathrm{t}_{\mathrm{R}}($ major enantiomer $)=12.604 \mathrm{~min}, 99 \%$ ee.
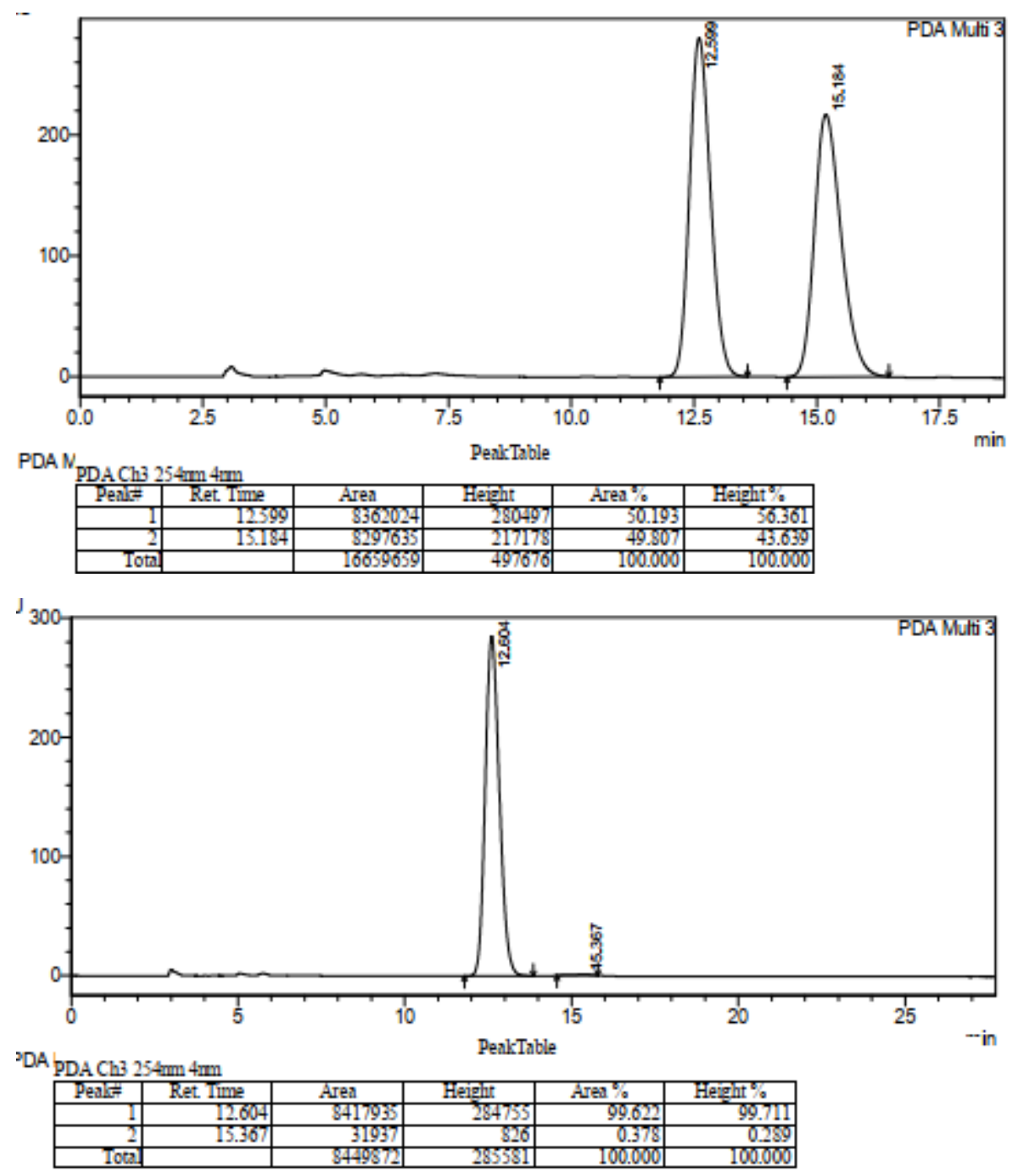
<smiles>O=C(c1ccccc1)N(Cc1ccccc1)C1([C@H]2CCS(=O)(=O)N2C(=O)c2ccc3ccccc3c2)CC[C@@H]1c1ccccc1</smiles>

Petroleum ether / ethyl acetate $=6: 1$ was used as the eluent for column chromatography. White solid (46.5 mg, 99\%), m. p. 198.5-199.6 ${ }^{\circ} \mathrm{C} .{ }^{1} \mathbf{H}$ NMR (500 MHz, $\left.\mathrm{CDCl}_{3}\right) \delta 8.48$ (s, 1H), 7.98 (d, J = 8.0 Hz, 1H), 7.91-7.82 (m, 3H), 7.61-7.52 ( m, 2H), 7.51-7.43 (m, 3H), 7.40-7.34 (m, 2H), 7.33-7.27 (m, 1H), 7.25-7.20 (m, 1H), 7.09 (t, $J=7.5 \mathrm{~Hz}, 1 \mathrm{H}), 6.74$ (d, $J=8.0 \mathrm{~Hz}, 1 \mathrm{H}), 5.03$ (s, 2H), 4.20-4.10 (m, 1H), 3.50 (dd, $J=12.0,5.5 \mathrm{~Hz}, 1 \mathrm{H}$ ), 2.85 (td, $J=14.0,6.0 \mathrm{~Hz}, 1 \mathrm{H}), 2.65$ (dd, $J=14.0,6.5 \mathrm{~Hz}, 1 \mathrm{H}) .{ }^{13} \mathbf{C} \mathbf{~ N M R}$ $\left(126 \mathrm{MHz} \mathrm{CDCl}_{3}\right) \delta 174.72,167.92,142.86,135.45,135.42,132.17,130.64,130.26,130.21,129.50$, 128.93, 128.42, 128.36, 127.88, 127.79, 127.33, 126.98, 126.86, 124.62, 123.56, 122.37, 109.95, 65.82, 47.18, 44.38, 29.71. HRMS ESI (m/z): Calcd for $\mathrm{C}_{30} \mathrm{H}_{24} \mathrm{~N}_{2} \mathrm{O}_{4} \mathrm{~S}$, [M+Na] ${ }^{+}$, 531.1349; found: 531.1360. $[\boldsymbol{\alpha}]_{\mathrm{D}}{ }^{20}=+7.2$ (c 1.0, $\mathrm{CH}_{2} \mathrm{Cl}_{2}$ ). Chiral HPLC analysis (Chiral pak AD-H, $i-\mathrm{PrOH} / n$-hexane = $20: 80$, flow rate $=0.5 \mathrm{~mL} / \mathrm{min}$, wave length $=254 \mathrm{~nm}), \mathrm{t}_{\mathrm{R}}($ minor enantiomer $)=58.467 \mathrm{~min}, \mathrm{t}_{\mathrm{R}}($ major enantiomer $)=$ $64.655 \mathrm{~min}, 99 \%$ ee. 

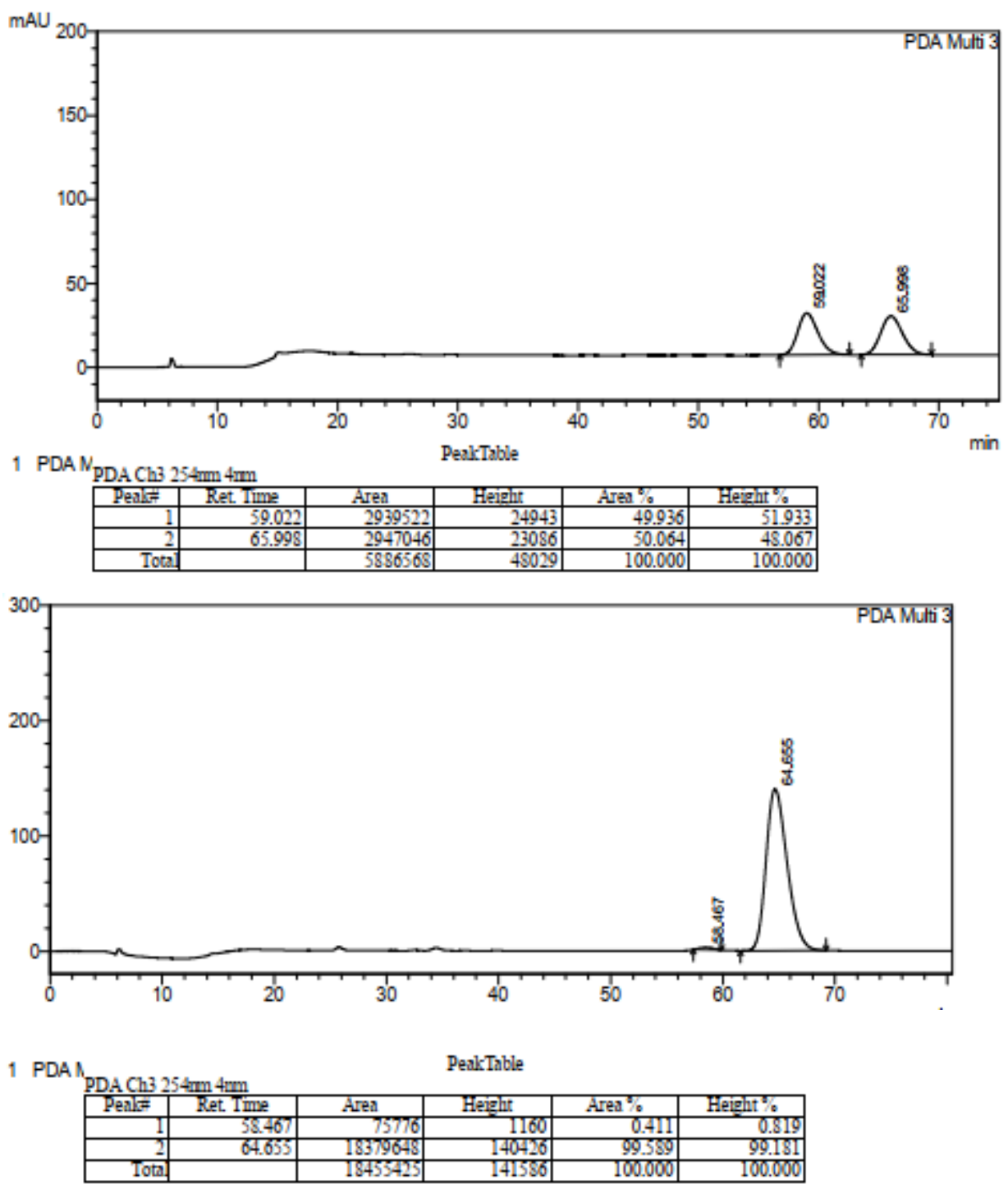

\section{Typical procedures and characterization data for compounds 6ab-6ai}

\subsection{General procedure for the synthesis of compounds 6ab-6ai}<smiles>[R4]C=CS(=O)(=O)F</smiles>

A solution of 3-benzamido-2-oxindole 1a (34.2 $\mathrm{mg}, 0.1 \mathrm{mmol}), \beta$-arylethenesulfonyl fluorides $\mathbf{2 b}-\mathbf{2} \mathbf{j}$ $(0.12 \mathrm{mmol})$ and catalyst $4 \mathbf{d}(12.6 \mathrm{mg}, 0.02 \mathrm{mmol})$ in $\mathrm{CH}_{2} \mathrm{Cl}_{2}(1.0 \mathrm{~mL})$ were stirred at rt for $24 \mathrm{~h}$. After the solvent as evaporated under vacuum, the residue was purified by flash column chromatography over silica gel to afford the products 6ab-6ai.

5.2 General procedure for the synthesis of racemic compounds 6ab-6ai 


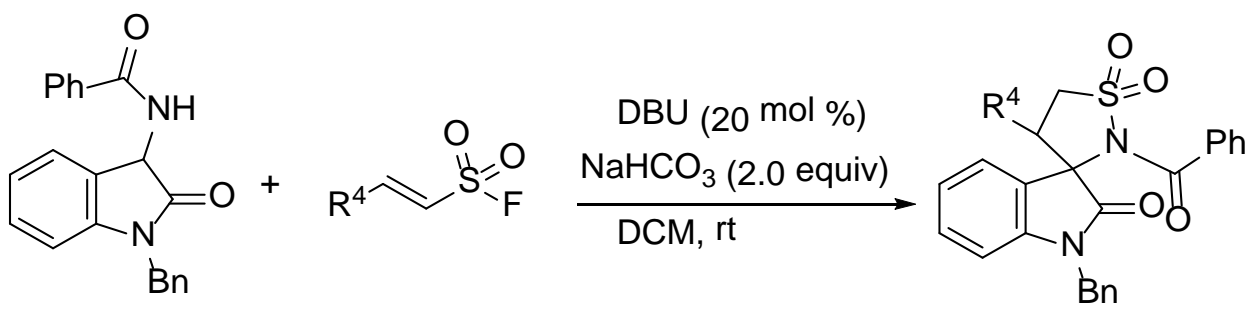

A solution of 3-benzamido-2-oxindole 1a (34.2 $\mathrm{mg}, 0.1 \mathrm{mmol}$ ), $\beta$-arylethenesulfonyl fluorides $\mathbf{2 b}$-2j (0.12 mmol), $\mathrm{NaHCO}_{3}(16.8 \mathrm{mg}, 0.2 \mathrm{mmol})$ and $\mathrm{DBU}$ ( $\left.3 \mathrm{mg}, 0.02 \mathrm{mmol}\right)$ in $\mathrm{CH}_{2} \mathrm{Cl}_{2}(1.0 \mathrm{~mL})$ was stirred at $\mathrm{rt}$ for $24 \mathrm{~h}$. After the solvent was evaporated under vacuum, the residue was purified by flash column chromatography over silica gel to afford the racemic products 6ab-6ai.

\subsection{Scale-up synthesis of compound $6 \mathrm{ab}$}<smiles>O=C(NC1C(=O)N(Cc2ccccc2)c2ccccc21)c1ccccc1</smiles>

A solution of 3-benzamido-2-oxindole $\mathbf{1 a}$ ( $342 \mathrm{mg}, 1.5 \mathrm{mmol}$ ), $\beta$-arylethenesulfonyl fluorides $\mathbf{2 b}$ (335 mg, $1.8 \mathrm{mmol}$ ) and catalyst $\mathbf{4 d}$ (189 mg, $0.3 \mathrm{mmol})$ in $\mathrm{CH}_{2} \mathrm{Cl}_{2}(15 \mathrm{~mL})$ were stirred at rt for $36 \mathrm{~h}$. After the solvent as evaporated under vacuum, the residue was purified by flash column chromatography over silica gel (petroleum ether / ethyl acetate $=8: 1$ ) to afford the product $\mathbf{6 a b}$ as a white solid (370 $\mathrm{mg}$, $73 \%)$.

\subsection{Characterizations of product 6ab-6ai}

(3S,4'S)-2'-benzoyl-1-benzyl-4'-phenylspiro[indoline-3,3'-isothiazolidin]-2-one 1',1'-dioxide (6ab)<smiles>O=C(c1ccccc1)N1C(=O)[C@H](c2ccccc2)CS1(=O)=O</smiles><smiles>[10BH2]c1ccccc1</smiles>

Petroleum ether / ethyl acetate $=8: 1$ was used as the eluent for column chromatography. White solid (41 mg, 80\%), m. p. 249.9-251.9 ${ }^{\circ} \mathrm{C} .{ }^{1} \mathbf{H}$ NMR (400 MHz, $\left.\mathrm{CDCl}_{3}\right) \delta$ 7.79-7.73 (m, 2H), 7.61-7.52 (m, 2H), 7.48-7.41 (m, 2H), 7.24-7.06 (m, 8H), 6.92-6.85 (m, 4H), 6.36 (d, $J=7.2 \mathrm{~Hz}, 1 \mathrm{H}), 4.80-4.70$ (m, 2H), 4.49 (dd, $J=14.8,6.0 \mathrm{~Hz}, 1 \mathrm{H}), 4.25-4.17$ (m, 1H), 3.87 (dd, $J=12.8,6.0 \mathrm{~Hz}, 1 \mathrm{H}) .{ }^{13} \mathbf{C}$ NMR $(101$ $\left.\mathrm{MHz}, \mathrm{CDCl}_{3}\right) \delta 171.74,167.61,142.81,134.73,133.20,132.19,130.10,129.92,128.92,128.68,128.61$, 128.47, 128.10, 127.38, 126.75, 124.72, 122.94, 122.81, 110.02, 71.88, 52.77, 46.16, 44.30. HRMS ESI

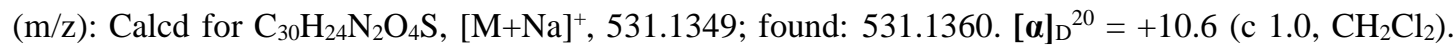
Chiral HPLC analysis (Chiral pak IG, $i-\mathrm{PrOH} / n$-hexane $=20: 80$, flow rate $=1.0 \mathrm{~mL} / \mathrm{min}$, wave length $=254 \mathrm{~nm}), \mathrm{t}_{\mathrm{R}}($ minor enantiomer $)=22.146 \mathrm{~min}, \mathrm{t}_{\mathrm{R}}($ major enantiomer $)=34.353 \mathrm{~min}, 87 \%$ ee . 


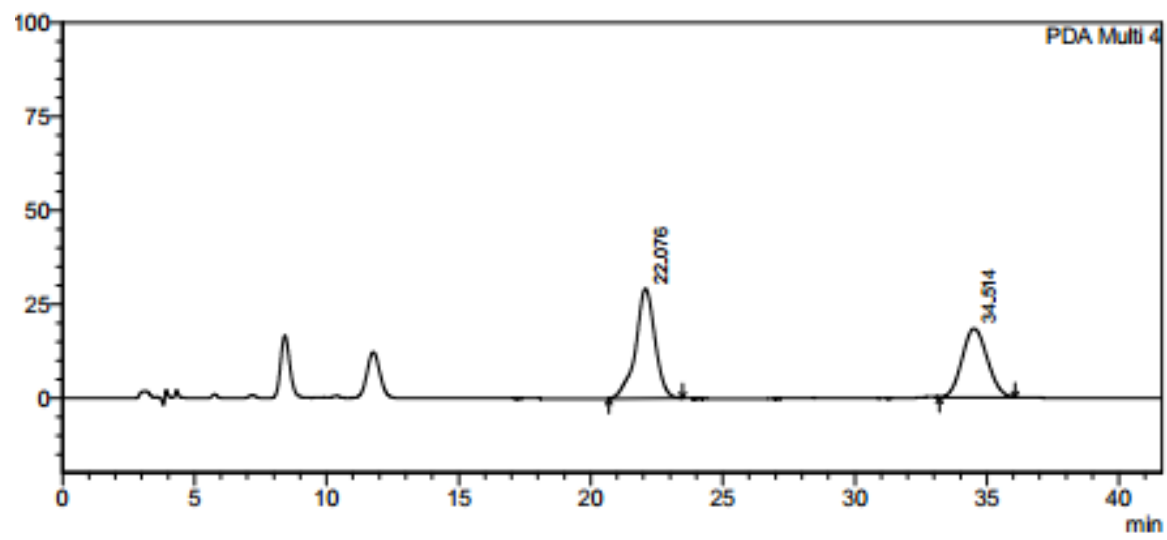

1 PDA Multi 4/254m 4nm
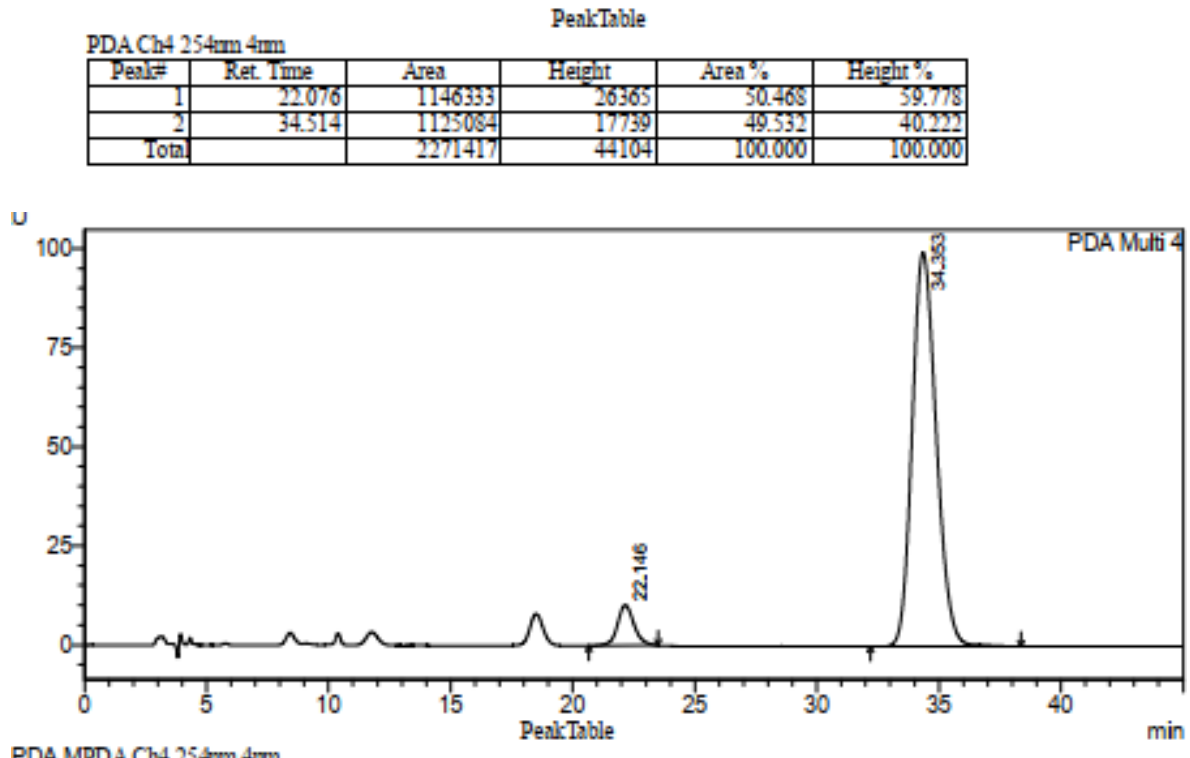

PDA MPDA Ch4 254m 4m

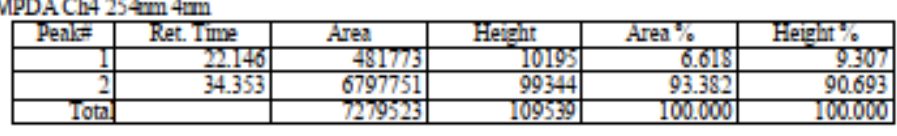

(3S,4' S)-2'-benzoyl-1-benzyl-4' '(4-chlorophenyl)spiro[indoline-3,3'-isothiazolidin]-2-one1',1'dioxide (6ac)<smiles>O=C1Nc2ccccc2[C@]2(C[C@@H](c3ccc(Cl)cc3)S(=O)(=O)C2)N1C(=O)c1ccccc1</smiles>

6ac ${ }^{\mathrm{Bn}}$

Petroleum ether / ethyl acetate $=8: 1$ was used as the eluent for column chromatography. White solid (40 mg, 74\%), m. p. 223.5-225.3 ${ }^{\circ} \mathrm{C} .{ }^{1} \mathbf{H}$ NMR (400 MHz, $\left.\mathrm{CDCl}_{3}\right) \delta$ 7.78-7.72 (m, 2H), 7.60-7.51 (m, 2H), 7.47-7.41 (m, 2H), 7.25-7.15 (m, 6H), 7.11 (td, $J=7.6,0.8 \mathrm{~Hz}, 1 \mathrm{H}), 6.91-6.84$ (m, 2H), 6.74-6.69 (m, 2H), 6.46 (d, $J=7.6 \mathrm{~Hz}, 1 \mathrm{H}$ ), 4.86 (d, $J=16.0 \mathrm{~Hz}, 1 \mathrm{H}$ ), 4.67 (d, $J=16.0 \mathrm{~Hz}, 1 \mathrm{H}$ ), 4.43 (dd, $J=14.4$, $5.6 \mathrm{~Hz}, 1 \mathrm{H}), 4.19-4.11(\mathrm{~m}, 1 \mathrm{H}), 3.85$ (dd, $J=12.8,6.0 \mathrm{~Hz}, 1 \mathrm{H}) .{ }^{13} \mathrm{C}$ NMR $\left(101 \mathrm{MHz}, \mathrm{CDCl}_{3}\right) \delta 171.54$, 167.58, 142.84, 134.64, 133.05, 132.30, 131.69, 130.42, 130.21, 128.96, 128.73, 128.46, 128.14, 127.59, 126.82, 124.37, 123.28, 122.98, 122.95, 110.23, 71.56, 52.66, 45.67, 44.34. HRMS ESI (m/z): Calcd 


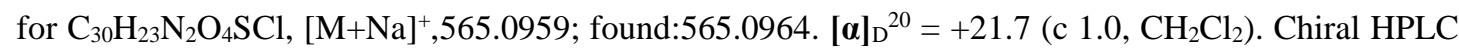
analysis (Chiral pak IG, $i$-PrOH $/ n$-hexane $=20: 80$, flow rate $=1.0 \mathrm{~mL} / \mathrm{min}$, wave length $=254 \mathrm{~nm}$ ), $\mathrm{t}_{\mathrm{R}}$ $($ minor enantiomer $)=29.4811 \mathrm{~min}, \mathrm{t}_{\mathrm{R}}($ major enantiomer $)=55.130 \mathrm{~min}, 78 \%$ ee.

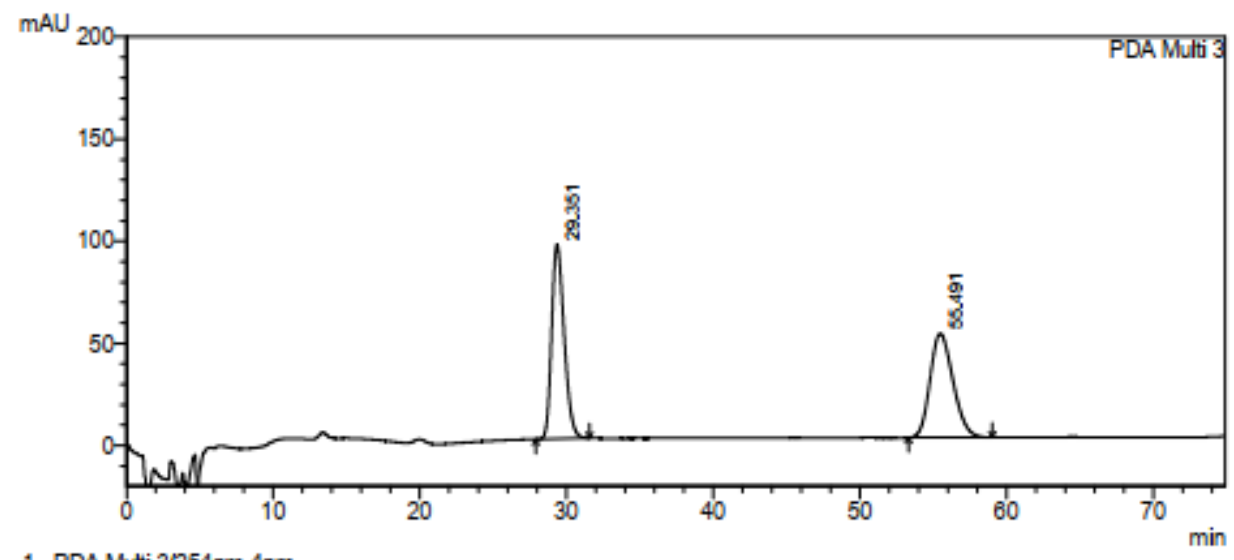

1 PDA Muti $3 / 254 \mathrm{rm} 4 \mathrm{~nm}$

PeakTable
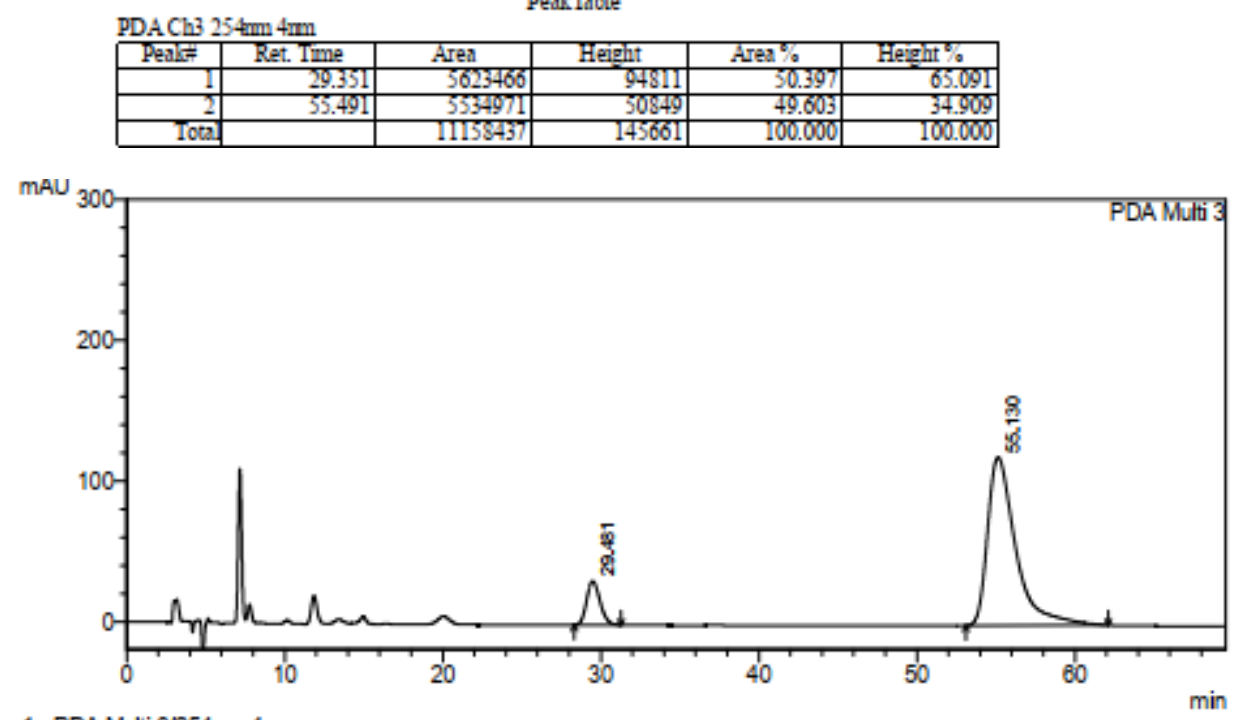

1 PDA Muti $3 / 254 \mathrm{rm} 4 \mathrm{~nm}$

PeakTable

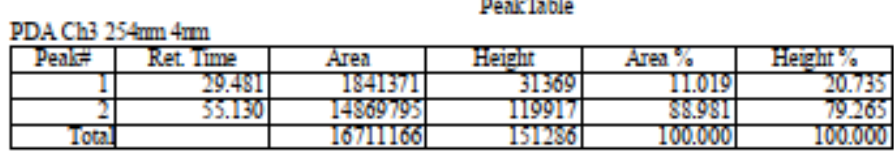

(3S,4'S)-2'-benzoyl-1-benzyl-4'-(4-bromophenyl)spiro[indoline-3,3'-isothiazolidin]-2-one 1',1'-dioxide (6ad)<smiles>O=C(c1ccccc1)N1[C@]2(C(=O)Nc3ccccc32)[C@H](c2ccc(Br)cc2)CS1(=O)=O</smiles>

6ad Bn

Petroleum ether / ethyl acetate $=8: 1$ was used as the eluent for column chromatography. White solid (43 mg, 73\%), m. p. 242.9-244.0 ${ }^{\circ} \mathrm{C} .{ }^{1} \mathbf{H}$ NMR $\left(400 \mathrm{MHz}, \mathrm{CDCl}_{3}\right) \delta$ 7.77-7.70 (m, 2H), 7.59-7.51 (m, 2H), 7.46-7.40 (m, 2H), 7.25-7.15 (m, 4H), 7.13-7.07( m, 1H), 7.06-7.00 (m, 2H), 6.90-6.84 (m, 2H), 6.80-6.74 (m, 2H), 6.45 (d, $J=8.0 \mathrm{~Hz}, 1 \mathrm{H}), 4.84$ (d, $J=16.0 \mathrm{~Hz}, 1 \mathrm{H}), 4.68$ (d, $J=16.4 \mathrm{~Hz}, 1 \mathrm{H}), 4.44$ 
(dd, $J=14.4,5.6 \mathrm{~Hz}, 1 \mathrm{H}), 4.19-4.09(\mathrm{~m}, 1 \mathrm{H}), 3.85$ (dd, $J=12.8,6.0 \mathrm{~Hz}, 1 \mathrm{H}) .{ }^{13} \mathrm{C}$ NMR (101 MHz, $\left.\mathrm{CDCl}_{3}\right) \delta 171.55,167.59,142.83,135.08,134.65,133.07,132.30,130.40,129.93,128.73,128.70$, 128.46, 128.14, 127.59, 126.82, 124.40, 122.98, 122.95, 110.21, 71.64, 52.71, 45.60, 44.32. HRMS

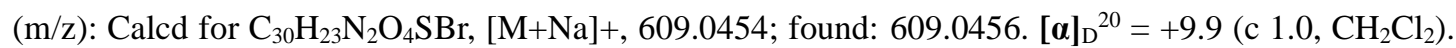
Chiral HPLC analysis (Chiral pak IG, $i-\mathrm{PrOH} / n$-hexane $=20: 80$, flow rate $=1.0 \mathrm{~mL} / \mathrm{min}$, wave length $=254 \mathrm{~nm}), \mathrm{t}_{\mathrm{R}}($ minor enantiomer $)=31.598 \mathrm{~min}, \mathrm{t}_{\mathrm{R}}($ major enantiomer $)=58.984 \mathrm{~min}, 82 \%$ ee.

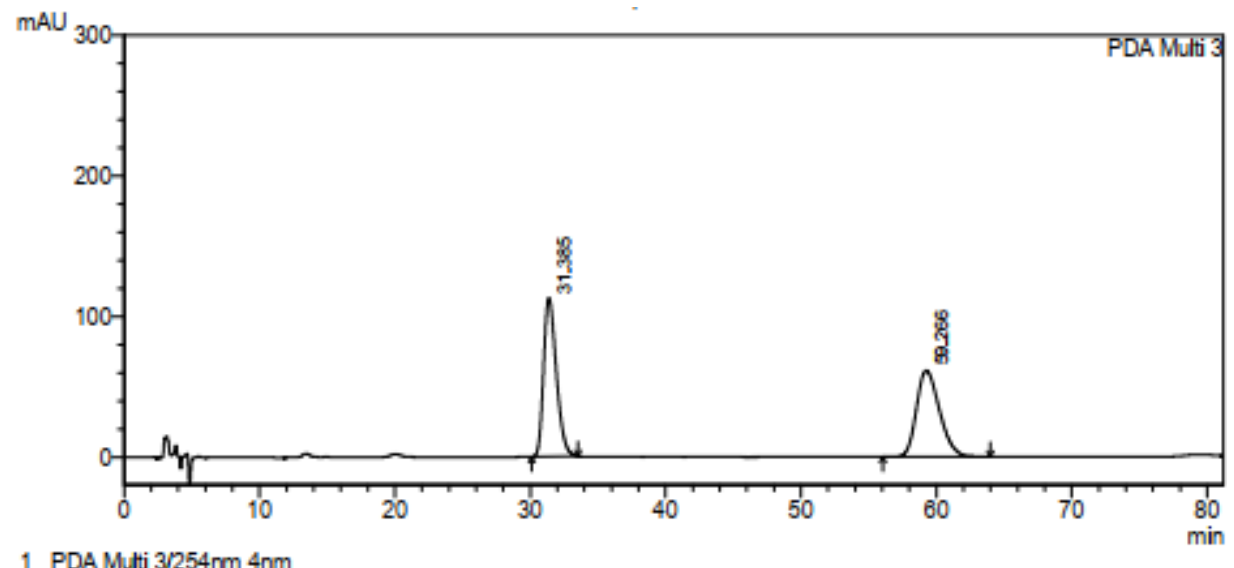

1 PDA Multi $3 / 254 \mathrm{rm} 4 \mathrm{~nm}$
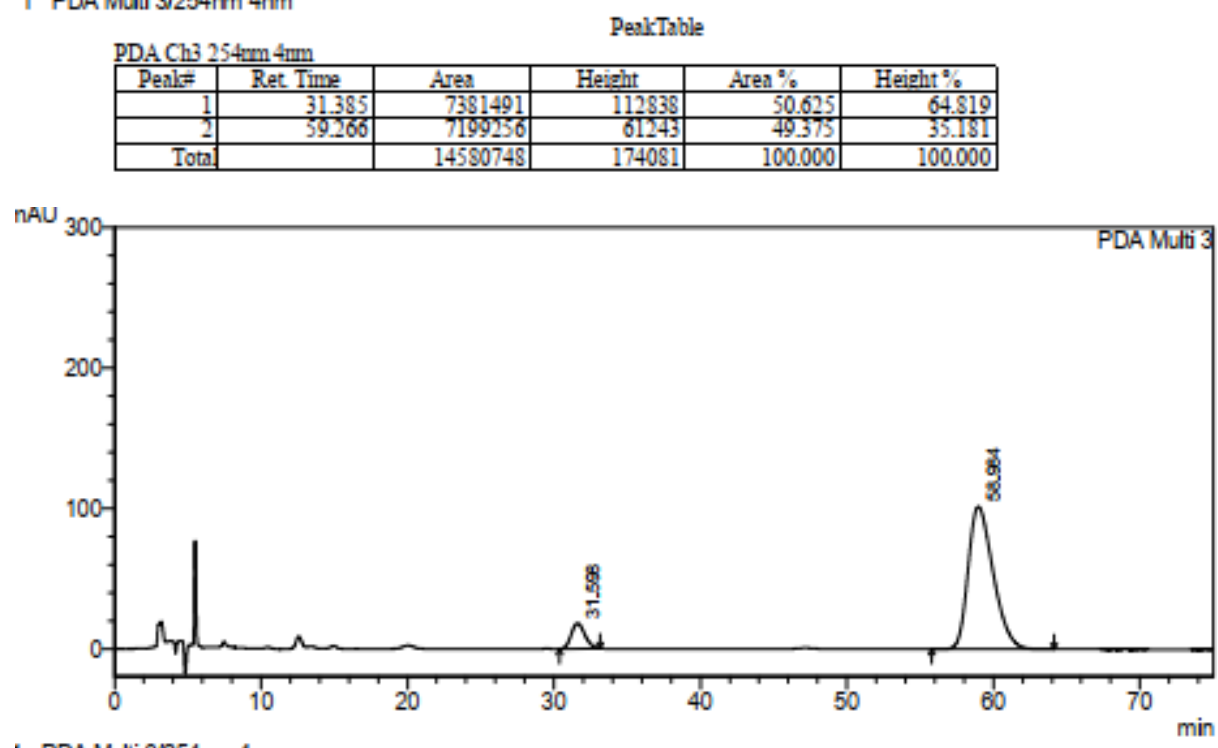

PDA Muti $3 / 254 \mathrm{~mm} 4 \mathrm{~nm}$

PeakTable

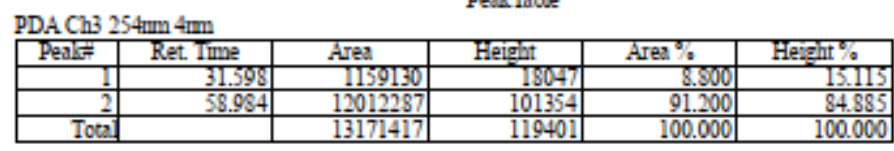

(3S,4'S)-2'-benzoyl-1-benzyl-4'-(3-bromophenyl)spiro[indoline-3,3'-isothiazolidin]-2-one 1',1'dioxide (6ae)<smiles>O=C(c1ccccc1)N1[C@]2(C(=O)N(Cc3ccccc3)c3ccccc32)[C@@H](c2cccc(Br)c2)CS1(=O)=O</smiles>

Petroleum ether / ethyl acetate $=8: 1$ was used as the eluent for column chromatography. White solid 
(46 mg, 78\%), m. p. 255.7-256.8 ${ }^{\circ} \mathrm{C} .{ }^{1} \mathbf{H}$ NMR (400 MHz, $\left.\mathrm{CDCl}_{3}\right) \delta$ 7.80-7.72 (m, 2H), 7.59-7.51 (m, 2H), 7.48-7.41 (m, 2H), 7.35 (dd, $J=8.0,0.8 \mathrm{~Hz}, 1 \mathrm{H}), 7.24-7.08$ (m, 5H), 7.05 (t, $J=1.6 \mathrm{~Hz}, 1 \mathrm{H}), 6.99-$ 6.89 (m, 3H), 6.78 (d, $J=8.0 \mathrm{~Hz}, 1 \mathrm{H}), 6.42$ (d, $J=7.6 \mathrm{~Hz}, 1 \mathrm{H}), 4.78$ (q, $J=16.0 \mathrm{~Hz}, 2 \mathrm{H}), 4.43$ (dd, $J=$ 14.4, $6.0 \mathrm{~Hz}, 1 \mathrm{H}), 4.21-4.10(\mathrm{~m}, 1 \mathrm{H}), 3.86$ (dd, $J=12.8,6.0 \mathrm{~Hz}, 1 \mathrm{H}) .{ }^{13} \mathrm{C} \mathrm{NMR}\left(101 \mathrm{MHz}, \mathrm{CDCl}_{3}\right) \delta$ 171.57, 167.55, 142.71, 134.65, 133.07, 132.29, 132.10, 131.71, 130.40, 129.87, 128.81, 128.46, 128.15, 127.53, 127.15, 126.74, 124.34, 123.00, 122.89, 122.45, 110.24, 71.65, 52.54, 45.66, 44.40. HRMS ESI

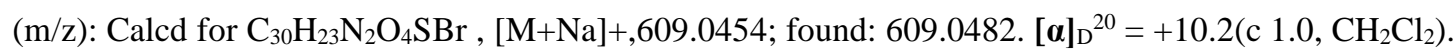
Chiral HPLC analysis (Chiral pak IG, $i-\mathrm{PrOH} / n$-hexane $=20: 80$, flow rate $=1.0 \mathrm{~mL} / \mathrm{min}$, wave length $=254 \mathrm{~nm}), \mathrm{t}_{\mathrm{R}}$ (minor enantiomer $)=18.496 \mathrm{~min}, \mathrm{t}_{\mathrm{R}}$ (major enantiomer $)=48.321 \mathrm{~min}, 95 \%$ ee .
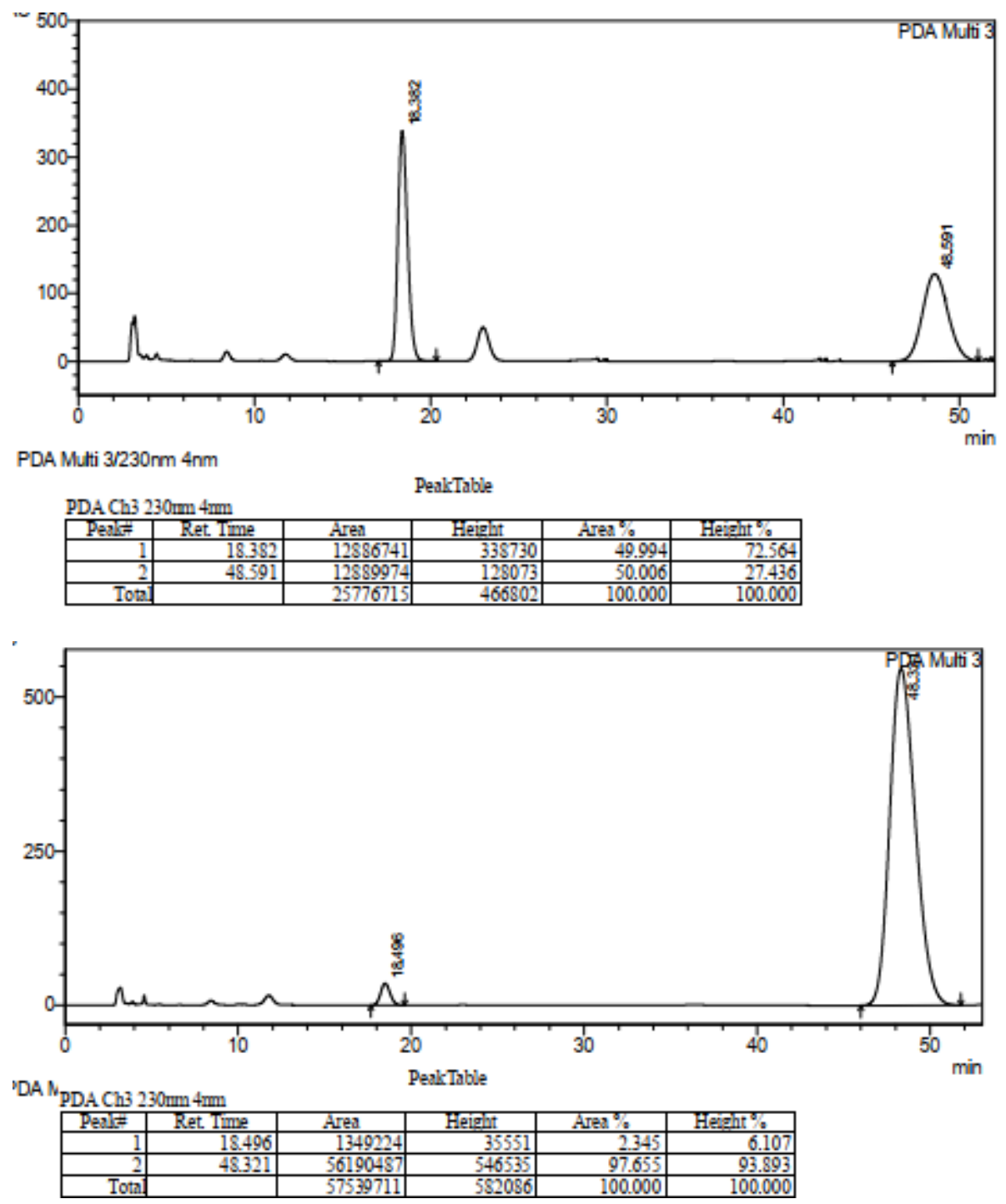

(3S,4'S)-2'-benzoyl-1-benzyl-4'-(3-methoxyphenyl)spiro[indoline-3,3'-isothiazolidin]-2-one 1',1'dioxide (6af) 
<smiles>COc1cccc([C@@H]2CS(=O)(=O)N(C(=O)c3ccccc3)[C@@]23C(=O)N(Cc2ccccc2)c2ccccc23)c1</smiles>

Petroleum ether / ethyl acetate $=8: 1$ was used as the eluent for column chromatography. White solid (37 mg, 69\%), m. p. $233.4-234.8^{\circ} \mathrm{C} .{ }^{1} \mathbf{H}$ NMR (400 MHz, $\left.\mathrm{CDCl}_{3}\right) \delta$ 7.94-7.88 (m, 2H), 7.61-7.55 (m, 2H), 7.50-7.44(m, 2H), 7.19 -7.08 (m, 6H), 6.86 (dd, $J=8.0,2.0 \mathrm{~Hz}, 1 \mathrm{H}$ ), 6.66 (d, $J=6.3 \mathrm{~Hz}, 2 \mathrm{H}$ ), 6.53 (d, $J=7.6 \mathrm{~Hz}, 1 \mathrm{H}), 6.38$ (d, $J=6.8 \mathrm{~Hz}, 1 \mathrm{H}), 6.29-6.24$ (m, 1H), 4.85 (d, $J=16.4 \mathrm{~Hz}, 1 \mathrm{H}), 4.80-4.71(\mathrm{~m}$, 1H), 4.47 (d, $J=16.0 \mathrm{~Hz}, 1 \mathrm{H}$ ), 4.36 (dd, $J=14.4,4.8 \mathrm{~Hz}, 1 \mathrm{H}$ ), 3.59 (dd, $J=11.6,4.8 \mathrm{~Hz}, 1 \mathrm{H}$ ), 3.48 (s, 3H). ${ }^{13}$ C NMR (101 MHz, $\left.\mathrm{CDCl}_{3}\right) \delta 172.61,167.97,159.52,144.09,134.97,133.63,133.07,131.41$, 130.38, 129.74, 128.87, 128.62, 128.43, 127.26, 126.49, 125.62, 123.27, 122.07, 120.32, 115.42, 113.48, 109.90, 70.95, 55.10, 50.02, 45.87, 44.25. HRMS ESI (m/z): Calcd for $\mathrm{C}_{31} \mathrm{H}_{26} \mathrm{~N}_{2} \mathrm{O}_{5} \mathrm{~S}$, [M+Na] 561.1455; found: 561.1465. $[\boldsymbol{\alpha}]_{\mathrm{D}}{ }^{20}=+18.1$ (c 1.0, $\mathrm{CH}_{2} \mathrm{Cl}_{2}$ ). Chiral HPLC analysis (Chiral pak IG, $i-$ $\mathrm{PrOH} / n$-hexane $=20: 80$, flow rate $=1.0 \mathrm{~mL} / \mathrm{min}$, wave length $=254 \mathrm{~nm}), \mathrm{t}_{\mathrm{R}}($ minor enantiomer $)=$ $47.479 \mathrm{~min}, \mathrm{t}_{\mathrm{R}}$ (major enantiomer) $=81.031 \mathrm{~min}, 87 \%$ ee.

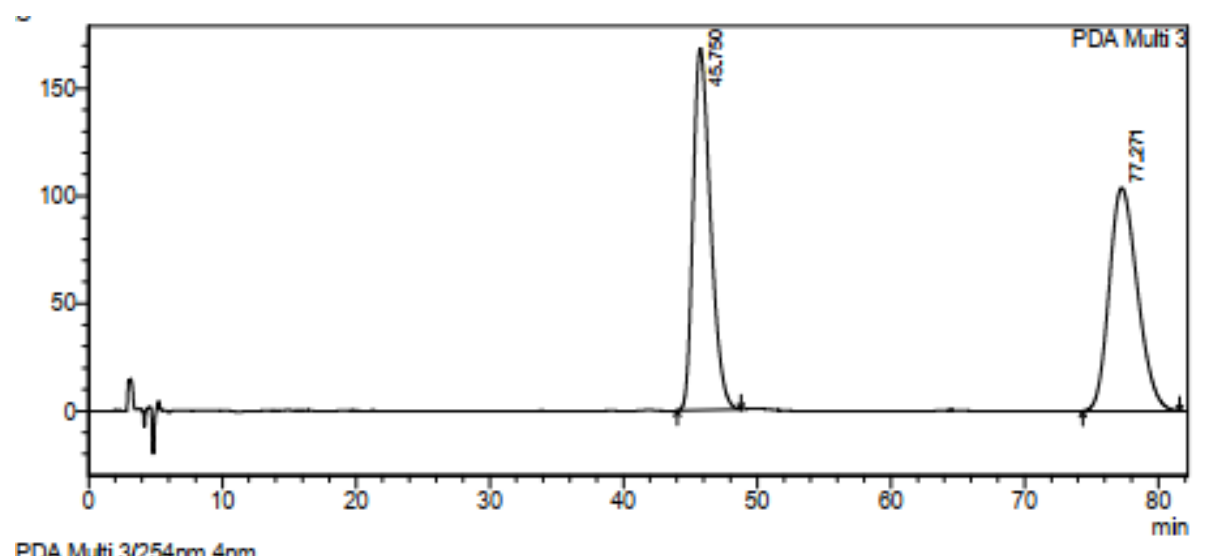

PDA Multi $3 / 254 \mathrm{~mm} 4 \mathrm{~nm}$

PeakTable

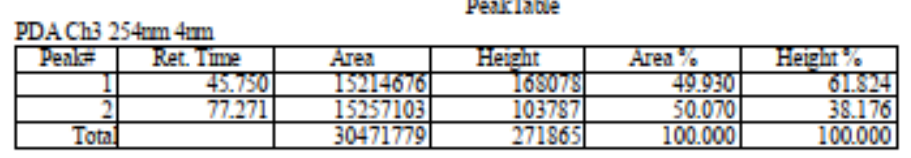




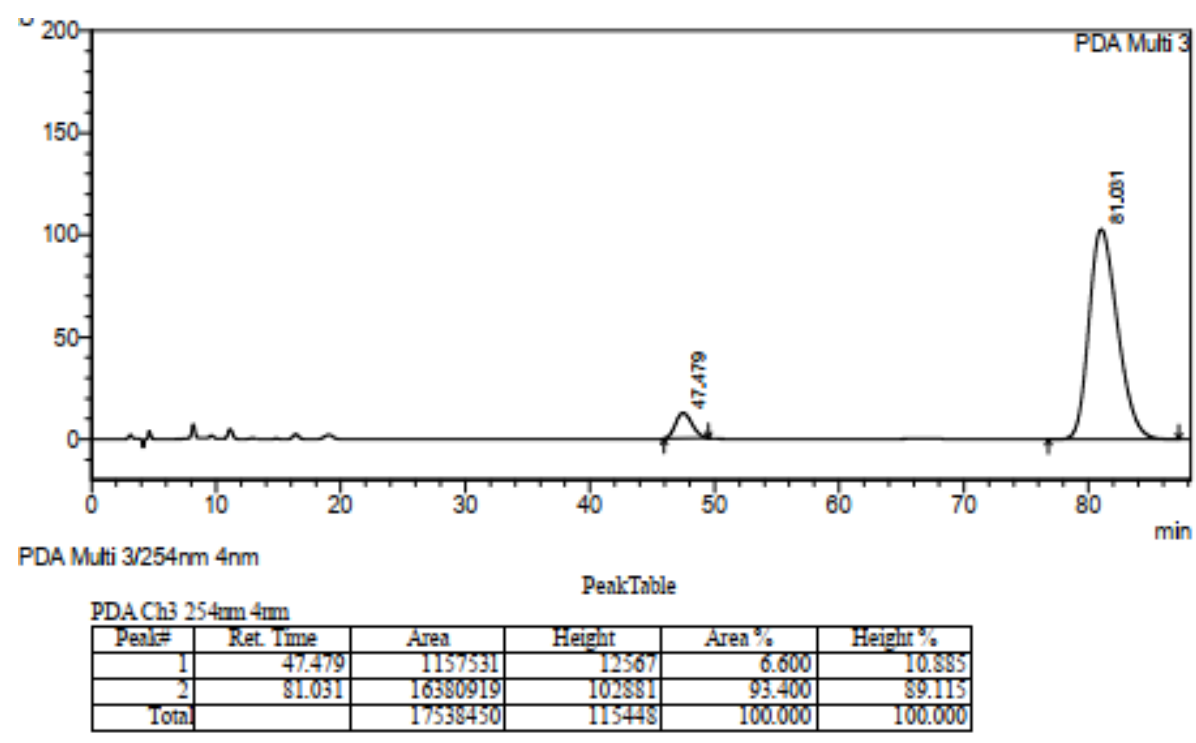

(3S,4' S)-2'-benzoyl-1-benzyl-4'-(3,5-bis(trifluoromethyl)phenyl)spiro[indoline-3,3'isothiazolidin]-2-one 1',1'-dioxide (6ag)<smiles>O=C(Cc1ccccc1)N1c2ccccc2[C@@]2(C(=O)N(Cc3ccccc3)c3ccccc3)[C@H](c3cc(C(F)(F)F)cc(C(F)(F)F)c3)CS(=O)(=O)N12</smiles>

Petroleum ether / ethyl acetate $=8: 1$ was used as the eluent for column chromatography. White solid (40 mg, 62\%), m. p. 228.8-230.1 ${ }^{\circ} \mathrm{C} .{ }^{1} \mathbf{H}$ NMR (500 MHz, $\left.\mathrm{CDCl}_{3}\right) \delta 7.91$ (d, $J=7.5 \mathrm{~Hz}, 2 \mathrm{H}$ ), 7.75 (s, 1H), 7.64-7.57 (m, 2H), 7.49 (t, $J=7.5 \mathrm{~Hz}, 2 \mathrm{H}), 7.29-7.24$ (m, 2H), 7.22-7.14 (m, 6H), 6.81 (d, $J=7.5$ Hz, 2H), 6.52 (d, $J=7.5 \mathrm{~Hz}, 1 \mathrm{H}), 4.81(\mathrm{t}, J=13.0 \mathrm{~Hz}, 1 \mathrm{H}), 4.57-4.50$ (m, 2H), 4.46 (dd, $J=14.5,4.0$ $\mathrm{Hz}, 1 \mathrm{H}), 3.65$ (dd, $J=11.0,4.0 \mathrm{~Hz}, 1 \mathrm{H}) .{ }^{13} \mathrm{C}$ NMR $\left(126 \mathrm{MHz}, \mathrm{CDCl}_{3}\right) \delta 171.86,167.73,143.57,134.64$, 133.31, 133.28, 131.88 (q, JC-F $=33.7 \mathrm{~Hz}), 128.87,128.66,128.52,128.38,127.60,126.76,124.35$, 123.87, 123.68, 122.92, 122.18, 121.51, 110.07, 70.38, 49.23, 45.48, 44.16. HRMS ESI (m/z): Calcd for $\mathrm{C}_{32} \mathrm{H}_{22} \mathrm{~N}_{2} \mathrm{O}_{4} \mathrm{~F}_{6} \mathrm{~S}$, [M+Na]+, 667.1097; found: 667.1085. [a. $]_{\mathrm{D}}^{20}=+12.0$ (c 1.0, $\mathrm{CH}_{2} \mathrm{Cl}_{2}$ ). Chiral HPLC analysis (Chiral pak IG, $i-\mathrm{PrOH} / n$-hexane $=20: 80$, flow rate $=1.0 \mathrm{~mL} / \mathrm{min}$, wave length $=254 \mathrm{~nm}$ ), $\mathrm{t}_{\mathrm{R}}$ $($ minor enantiomer $)=5.308 \mathrm{~min}, \mathrm{t}_{\mathrm{R}}$ (major enantiomer $)=8.419 \mathrm{~min}, 71 \%$ ee. 


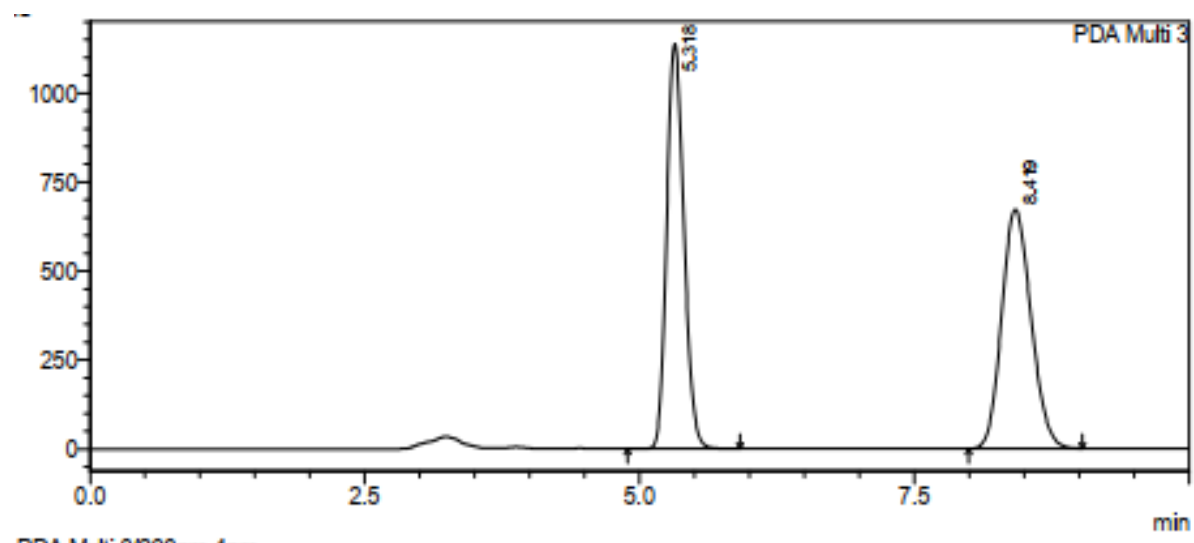

PDA Mutti $3 / 230 \mathrm{~mm} 4 \mathrm{~nm}$

PeakTable
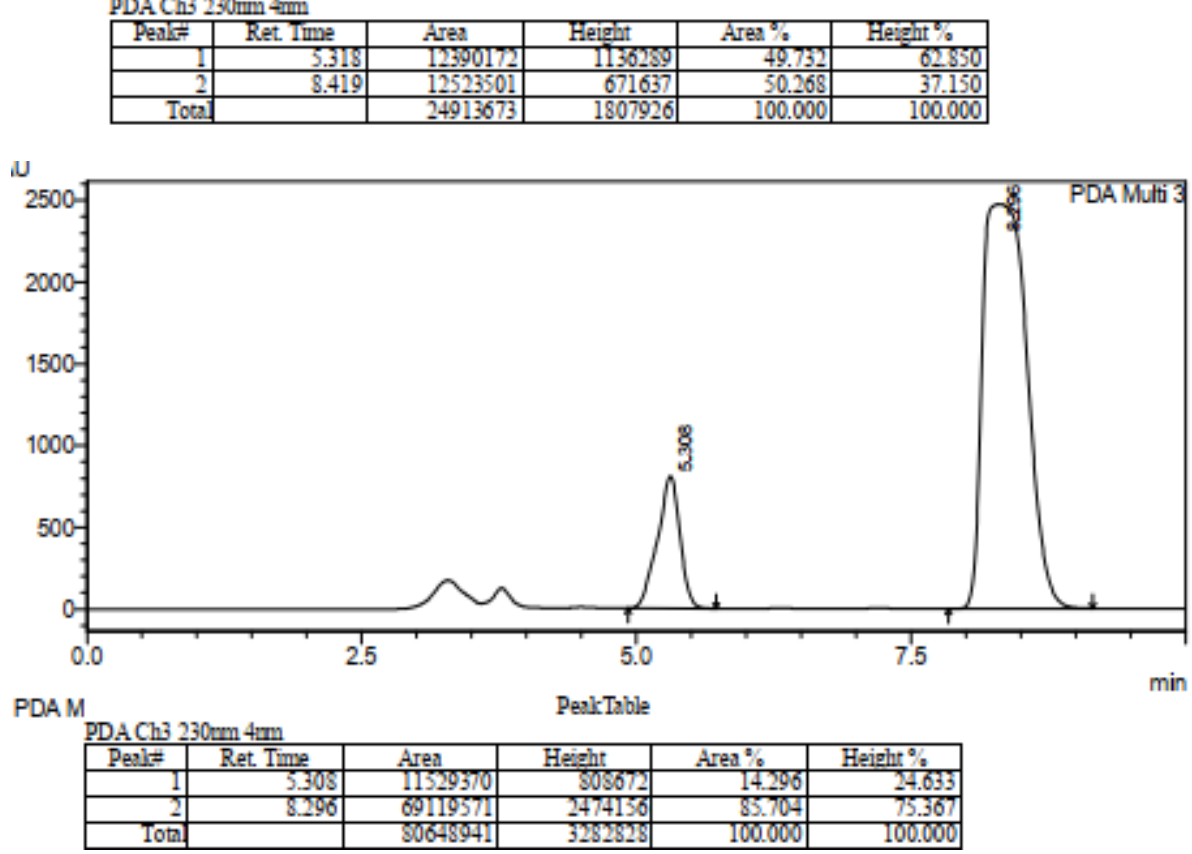

(3S,4' S)-2'-benzoyl-1-benzyl-4' -(thiophen-3-yl)spiro[indoline-3,3'-isothiazolidin]-2-one1',1'dioxide (6ah)<smiles>O=C(c1ccccc1)N1C(=O)[C@]2(c3ccccc31)[C@H](c1ccsc1)CC(=O)N2Cc1ccccc1</smiles>

Petroleum ether / ethyl acetate $=6: 1$ was used as the eluent for column chromatography. White solid (31 mg, 60\%), m. p. 209.5-211.4 ${ }^{\circ} \mathrm{C} .{ }^{1} \mathbf{H}$ NMR (400 MHz, $\left.\mathrm{CDCl}_{3}\right) \delta$ 7.88-7.71 (m, 2H), 7.61-7.53 (m, 2H), 7.47 (t, $J=7.6 \mathrm{~Hz}, 2 \mathrm{H}$ ), 7.30-7.19 (m, 5H), 7.03-6.97 (m, 2H), 7.03-6.97 (m, 2H), 6.91 (dd, $J=2.8$, $0.8 \mathrm{~Hz}, 1 \mathrm{H}$ ), 6.50 (d, $J=7.6 \mathrm{~Hz}, 1 \mathrm{H}), 6.47$ (dd, $J=5.2,1.2 \mathrm{~Hz}, 1 \mathrm{H}), 4.83$ (dd, $J=38.0,16.0 \mathrm{~Hz}, 2 \mathrm{H}$ ), 4.62 (dd, $J=14.0,6.0 \mathrm{~Hz}, 1 \mathrm{H}$ ), 4.17-4.06 (m, $1 \mathrm{H}), 3.93$ (dd, $J=12.8,6.4 \mathrm{~Hz}, 1 \mathrm{H}) .{ }^{13} \mathbf{C}$ NMR (101 MHz, $\left.\mathrm{CDCl}_{3}\right) \delta 171.88,167.70,142.94,134.81,133.12,132.28,130.90,130.25,128.78,128.52,128.12$, 127.48, 126.78, 126.67, 126.14, 125.14, 125.05, 122.90, 122.83, 110.09, 71.42, 53.47, 44.36, 41.96. HRMS ESI (m/z): Calcd for $\mathrm{C}_{28} \mathrm{H}_{22} \mathrm{~N}_{2} \mathrm{O}_{4} \mathrm{~S}_{2}$, [M+Na] $]^{+}$, 537.0913; found: 537.0908. [ $\left.\boldsymbol{\alpha}\right]_{\mathrm{D}}{ }^{20}=+19.2$ (c 1.0, 
$\mathrm{CH}_{2} \mathrm{Cl}_{2}$ ). Chiral HPLC analysis (Chiral pak IG, $i$-PrOH$/ n$-hexane $=20: 80$, flow rate $=1.0 \mathrm{~mL} / \mathrm{min}$, wave length $=254 \mathrm{~nm}), \mathrm{t}_{\mathrm{R}}($ minor enantiomer $)=45.420 \mathrm{~min}, \mathrm{t}_{\mathrm{R}}$ (major enantiomer $)=69.740 \mathrm{~min}, 91 \%$ ee.

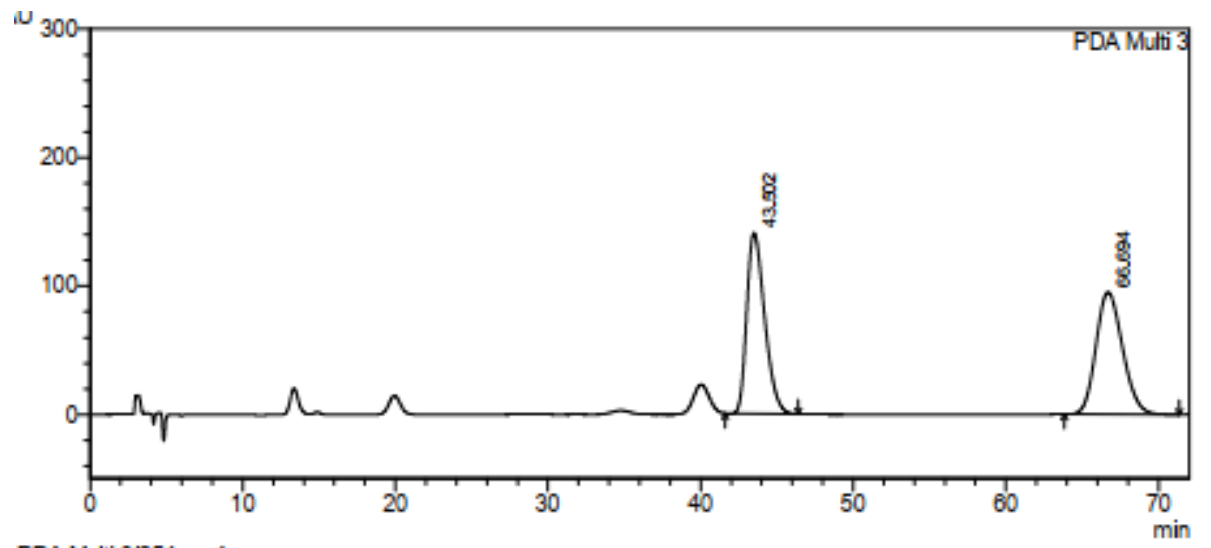

PDA Muti $3 / 254 \mathrm{~mm} 4 \mathrm{~nm}$

PeakTable
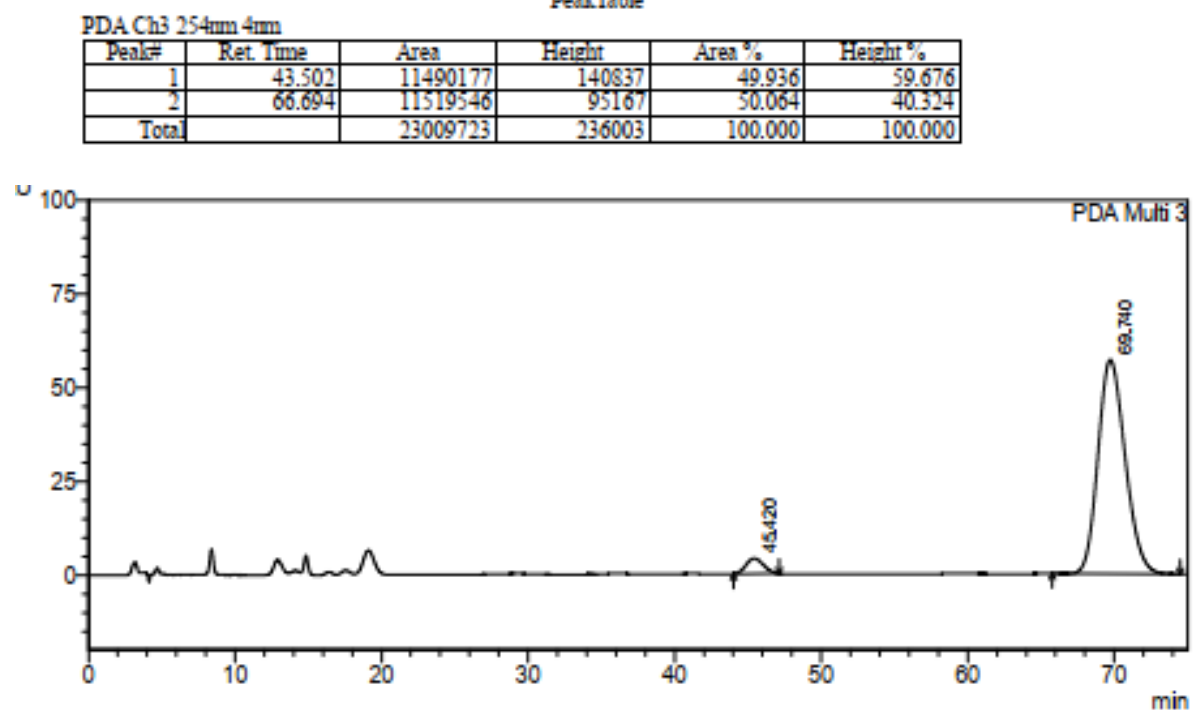

PDA Muti $3 / 254 \mathrm{~mm} 4 \mathrm{~nm}$

PeakTable

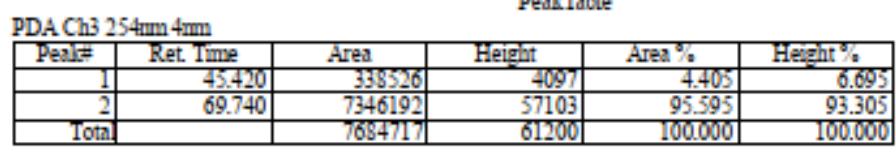

(3S,4'S)-2'-benzoyl-1-benzyl-4'-(thiophen-2-yl)spiro[indoline-3,3'-isothiazolidin]-2-one dioxide (6ai)<smiles>O=C(c1ccccc1)N1[C@]2(C(=O)Nc3ccccc32)[C@@H](c2cccs2)CS1(=O)=O</smiles>

6ai Bn

Petroleum ether / ethyl acetate $=6: 1$ was used as the eluent for column chromatography. White solid (29 mg, 57\%), m. p. 211.5-213.5 ${ }^{\circ} \mathrm{C} .{ }^{1} \mathbf{H}$ NMR (400 MHz, $\left.\mathrm{CDCl}_{3}\right) \delta$ 7.98-7.85 (m, 2H), 7.62-7.51 (m, 2H), 7.50-7.44 (m, 2H), 7.24-7.18 (m, 4H), 7.17-7.10 (m, 2H), 6.90 (dd, $J=5.2,3.6 \mathrm{~Hz}, 1 \mathrm{H}), 6.85-6.78$ (m, 2H), 6.74 (d, $J=3.6 \mathrm{~Hz}, 1 \mathrm{H}), 6.48$ (d, $J=8.0 \mathrm{~Hz}, 1 \mathrm{H}), 4.89$ (d, $J=16.0 \mathrm{~Hz}, 1 \mathrm{H}), 4.74$ (dd, $J=14.4$, 11.6 Hz, 1H), 4.64-4.52 (m, 2H), 3.69 (dd, $J=11.6,4.8 \mathrm{~Hz}, 1 \mathrm{H}) .{ }^{13} \mathrm{C}$ NMR $\left(101 \mathrm{MHz}, \mathrm{CDCl}_{3}\right) \delta 172.57$, 
168.00, 144.42, 135.05, 133.55, 133.12, 131.90, 130.64, 128.93, 128.65, 128.42, 127.31, 127.12, 127.03, 126.77, 126.23, 125.25, 123.38, 122.14, 109.88, 70.74, 50.88, 44.33, 42.01. HRMS ESI (m/z): Calcd

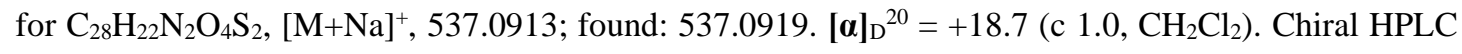
analysis (Chiral pak IG, $i-\mathrm{PrOH} / n$-hexane $=20: 80$, flow rate $=1.0 \mathrm{~mL} / \mathrm{min}$, wave length $=254 \mathrm{~nm}$ ), $\mathrm{t}_{\mathrm{R}}$ $($ minor enantiomer $)=41.459 \mathrm{~min}, \mathrm{t}_{\mathrm{R}}($ major enantiomer $)=61.873 \mathrm{~min}, 91 \%$ ee .

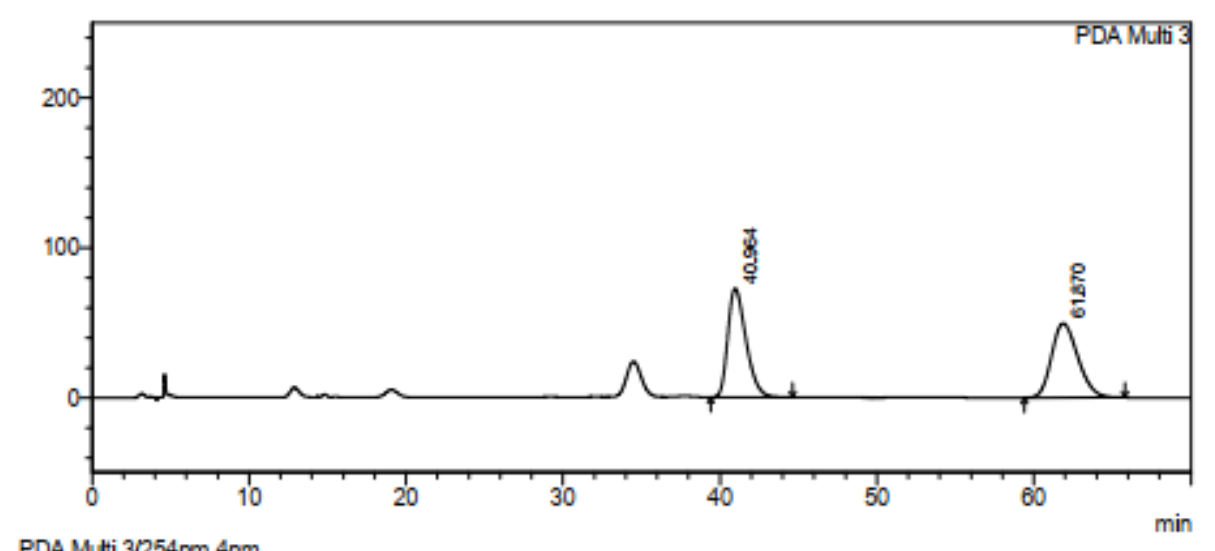

PDA Muti $3 / 254 \mathrm{~mm} 4 \mathrm{~nm}$
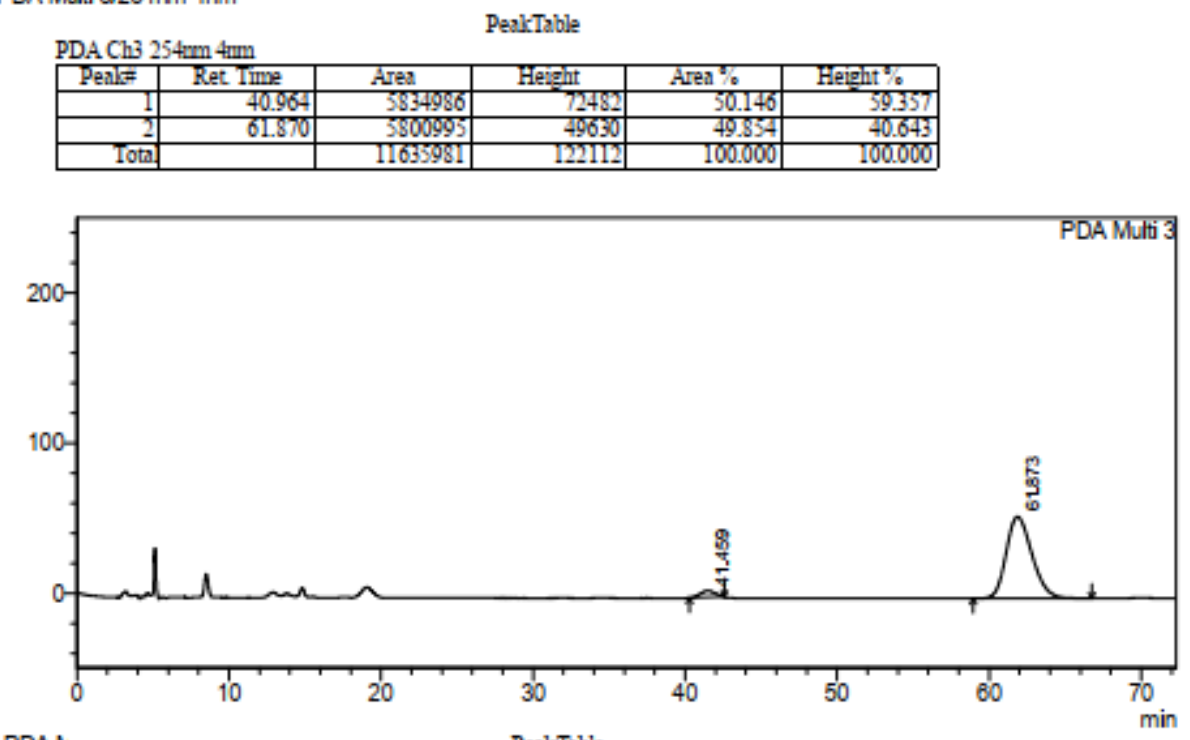

PDA N

PealkTable

\begin{tabular}{|c|c|c|c|c|c|}
\hline Pealk= & Ret Time & Areas & Heigint & Are: \% & 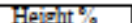 \\
\hline & 41.459 & 295758 & 4298 & 4.419 & 7.321 \\
\hline 2 & 61.875 & 6397758 & 54405 & 95.581 & 92679 \\
\hline$T O C_{2}$ & & 6693517 & 58703 & 100.000 & 100.000 \\
\hline
\end{tabular}

\section{Procedure for the gram-scale synthesis of $3 a$}<smiles>O=C(NC1C(=O)N(Cc2ccccc2)c2ccccc21)c1ccccc1</smiles>

$1 a$

(3.0 $\mathrm{mmol})$<smiles>C=CS(=O)(=O)F</smiles>

$2 a$

(3.3 $\mathrm{mmol})$<smiles>O=C(N[C@]1(CCS(=O)(=O)F)C(=O)N(Cc2ccccc2)c2ccccc21)c1ccccc1</smiles>

3a $1.3 \mathrm{~g}$ 96\% yield, 99\% ee

A solution of 3-benzamido-2-oxindole 1a (1.03 g, 3 mmol), ethylene sulfonyl fluoride 2a (396 mg, 3.6 mmol) and catalyst $\mathbf{4 d}$ (378 mg, $0.6 \mathrm{mmol})$ in $\mathrm{CH}_{2} \mathrm{Cl}_{2}(10 \mathrm{~mL})$ was stirred at rt for $24 \mathrm{~h}$. After the solvent 
was evaporated under vacuum, the residue was purified by flash column chromatography over silica gel (petroleum ether / ethyl acetate $=8: 1$ ) to afford the product 3a as a white solid (1.3 g, 96\%).

\section{Procedures for the transformations of $3 a$}

Synthesis of (S)-N-(1-benzyl-2-oxo-3-(2-(pyrrolidin-1-ylsulfonyl)ethyl)indolin-3-yl)benzamide (7a)

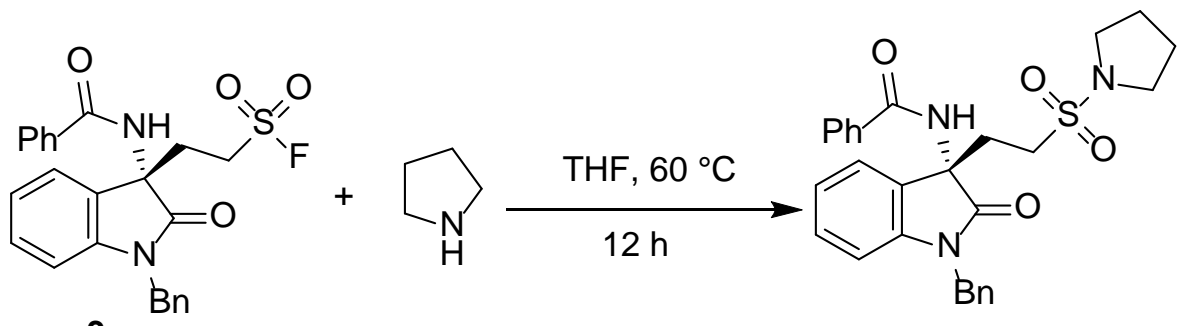

$3 a$

$7 \mathrm{a}, 70 \%$ yield, $99 \%$ ee

A solution of (S)-2-(3-benzamido-1-benzyl-2-oxoindolin-3-yl)ethane-1-sulfonyl fluoride 3a (45.2 mg, $0.1 \mathrm{mmol})$, pyrrolidine $(10.7 \mathrm{mg}, 0.15 \mathrm{mmol})$ in THF $(10 \mathrm{~mL})$ was stirred in an oil bath at $60{ }^{\circ} \mathrm{C}$ until the completion of the reaction (12 h) as indicated by TLC. After the solvent was evaporated under vacuum, the residue was purified by flash column chromatography over silica gel (petroleum ether / ethyl acetate $=8: 1$ ) to afford the product $7 \mathbf{a}$ as a white solid (35.2 mg, 70\%). m. p. $166.5-167.4{ }^{\circ} \mathrm{C} .{ }^{1} \mathbf{H}$ NMR (400 $\left.\mathrm{MHz} \mathrm{CDCl}_{3}\right) \delta 8.31(\mathrm{~s}, 1 \mathrm{H}), 7.92(\mathrm{~d}, J=7.2 \mathrm{~Hz}, 2 \mathrm{H}), 7.52-7.46(\mathrm{~m}, 1 \mathrm{H}), 7.46-7.39(\mathrm{~m}, 4 \mathrm{H}), 7.35(\mathrm{t}, J=$ $7.5 \mathrm{~Hz}, 2 \mathrm{H}), 7.28$ (d, $J=7.2 \mathrm{~Hz}, 2 \mathrm{H}), 7.19$ (t, $J=7.6 \mathrm{~Hz}, 1 \mathrm{H}), 7.02$ (t, $J=7.6 \mathrm{~Hz}, 1 \mathrm{H}), 6.74$ (d, $J=7.6$ $\mathrm{Hz}, 1 \mathrm{H}$ ), 5.02 (dd, $J=49.2,16.0 \mathrm{~Hz}, 2 \mathrm{H}), 3.82-3.72$ (m, 1H), 3.42-3.29 (m, 4H), 3.14-3.04 (m, 1H), 2.73-2.64 (m, 1H), 2.32-2.23 (m, 1H), 1.97-1.87 (m, 4H). ${ }^{13} \mathbf{C}$ NMR (101 MHz, $\left.\mathrm{CDCl}_{3}\right) \delta 175.95,166.26$, 142.21, 135.86, 132.59, 131.93, 129.95, 129.18, 128.88, 128.48, 127.61, 127.56, 127.25, 123.12, 122.25, 109.58, 59.62, 47.97, 44.24, 42.07, 30.14, 25.85. HRMS ESI (m/z): Calcd for $\mathrm{C}_{28} \mathrm{H}_{29} \mathrm{~N}_{3} \mathrm{O}_{4} \mathrm{~S}$, $[\mathrm{M}+\mathrm{Na}]^{+}$,

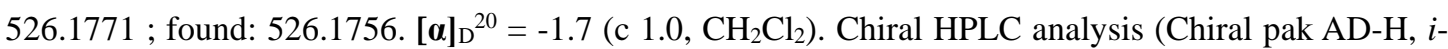
$\mathrm{PrOH} / n$-hexane $=20: 80$, flow rate $=0.5 \mathrm{~mL} / \mathrm{min}$, wave length $=254 \mathrm{~nm})$, $\mathrm{tR}$ (major enantiomer $)=$ $29.630 \mathrm{~min}$, tR (minor enantiomer) = $39.118 \mathrm{~min}, 99 \%$ ee.

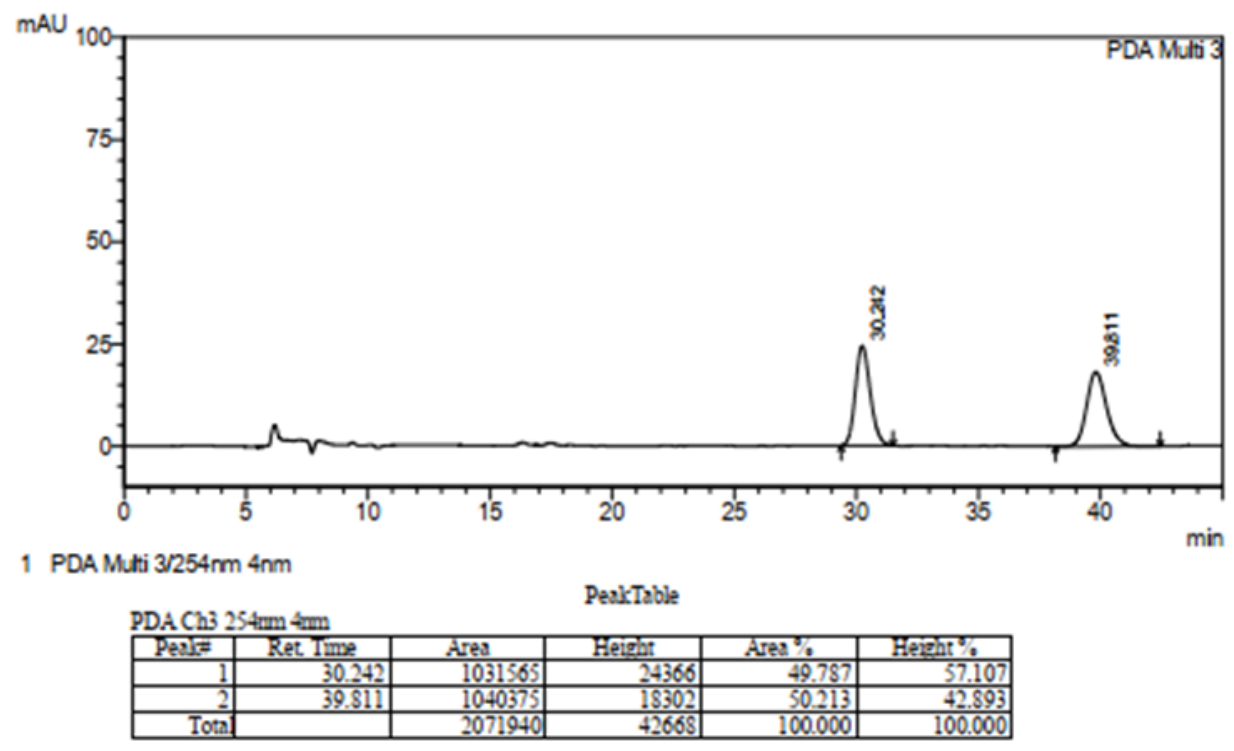




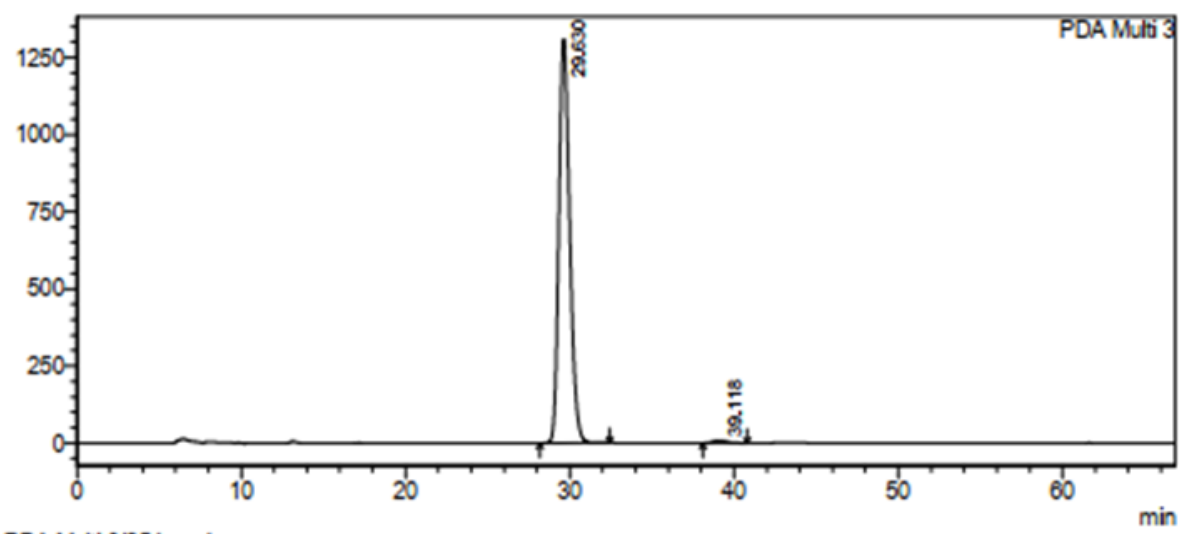

PDA Muti $3 / 254 \mathrm{~mm} 4 \mathrm{~nm}$

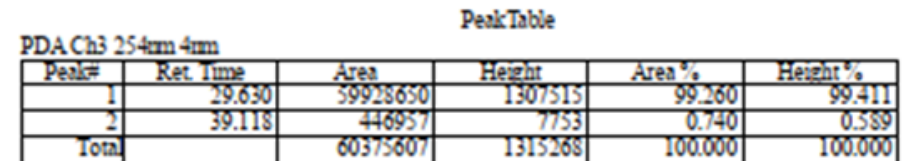

Synthesis of 4-methoxyphenyl (S)-2-(3-benzamido-1-benzyl-2-oxoindolin-3-yl)ethane-1-sulfonate (8a)<smiles>O=C(N[C@]1(CCS(=O)(=O)F)C(=O)N(Cc2ccccc2)c2ccccc21)c1ccccc1</smiles>

3a

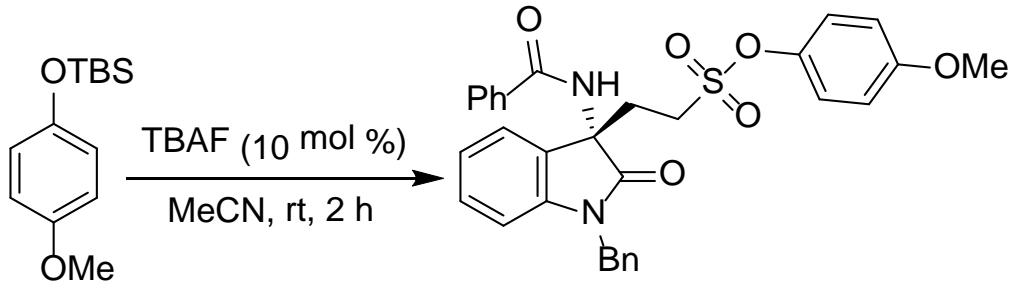

$\mathbf{8 a}, 75 \%$ yield, $99 \%$ ee

To a solution of (S)-2-(3-benzamido-1-benzyl-2-oxoindolin-3-yl)ethane-1-sulfonyl fluoride 3a (45.2 mg, $0.1 \mathrm{mmol})$, p-methoxyphenyl TBS ether $(35.7 \mathrm{mg}, 0.15 \mathrm{mmol})$ in MeCN $(1 \mathrm{~mL})$ was added tetrabutylammonium fluoride (TBAF) $(1.5 \mathrm{mg}, 0.01 \mathrm{mmol})$ at $0{ }^{\circ} \mathrm{C}$. The mixture was stirred at rt until the completion of the reaction $(2 \mathrm{~h})$ as indicated by TLC. After the solvent was evaporated under vacuum, the residue was purified by flash column chromatography over silica gel (petroleum ether / ethyl acetate $=8: 1)$ to afford the product 8a as a white solid $(41 \mathrm{mg}, 75 \%)$. m. p. $121.5-123.2^{\circ} \mathrm{C} .{ }^{1} \mathbf{H}$ NMR $(400$ $\left.\mathrm{MHz}, \mathrm{CDCl}_{3}\right) \delta$ 7.86-7.77 (m, 2H), 7.61 (s, $\left.1 \mathrm{H}\right), 7.53-7.47(\mathrm{~m}, 1 \mathrm{H}), 7.44-7.37$ (m, 4H), 7.36-7.29 (m, 3H), 7.30-7.24 (m, 2H), 7.23-7.12 (m, 3H), 7.04 (t, $J=7.2 \mathrm{~Hz}, 1 \mathrm{H}), 6.91-6.81$ (m, 2H), 6.76 (d, $J=8.0$ Hz, 1H), 5.00 (dd, $J=33.6$ Hz, 15.6 Hz, 2H), 3.83-3.72 (m, 4H), 3.33-3.23 (m, 1H), 2.87-2.78 (m, 1H), 2.53-2.43 (m, 1H). ${ }^{13} \mathrm{C}$ NMR (101 MHz, $\left.\mathrm{CDCl}_{3}\right) \delta 175.32,166.48,158.62,142.35,142.32,135.56$, 132.51, 132.13, 129.55, 128.93, 128.58, 127.74, 127.45, 127.28, 123.32, 123.06, 122.65, 115.00, 109.81, 59.78, 55.66, 44.37, 43.53, 30.69. HRMS ESI (m/z): Calcd for $\mathrm{C}_{31} \mathrm{H}_{28} \mathrm{~N}_{2} \mathrm{O}_{6} \mathrm{~S}$, $[\mathrm{M}+\mathrm{Na}]^{+}$, 579.1560; found: 579.1559. $[\boldsymbol{\alpha}]_{\mathrm{D}}{ }^{20}=+24.6$ (c 1.0, $\mathrm{CH}_{2} \mathrm{Cl}_{2}$ ). Chiral HPLC analysis (Chiral pak AD-H, $i-\mathrm{PrOH} / n$-hexane $=$ $20: 80$, flow rate $=0.5 \mathrm{~mL} / \mathrm{min}$, wave length $=254 \mathrm{~nm}$ ), $\mathrm{tR}$ (major enantiomer $)=31.981 \mathrm{~min}, \mathrm{tR}$ (minor enantiomer) $=51.900 \mathrm{~min}, 99 \%$ ee. 


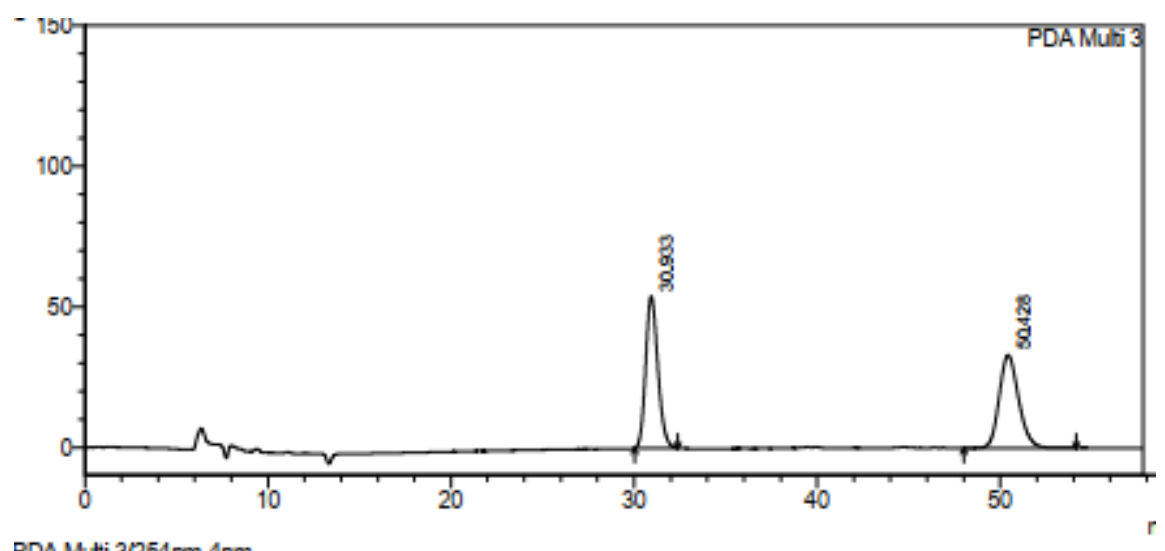

PDA Muti 3/254nm 4nm
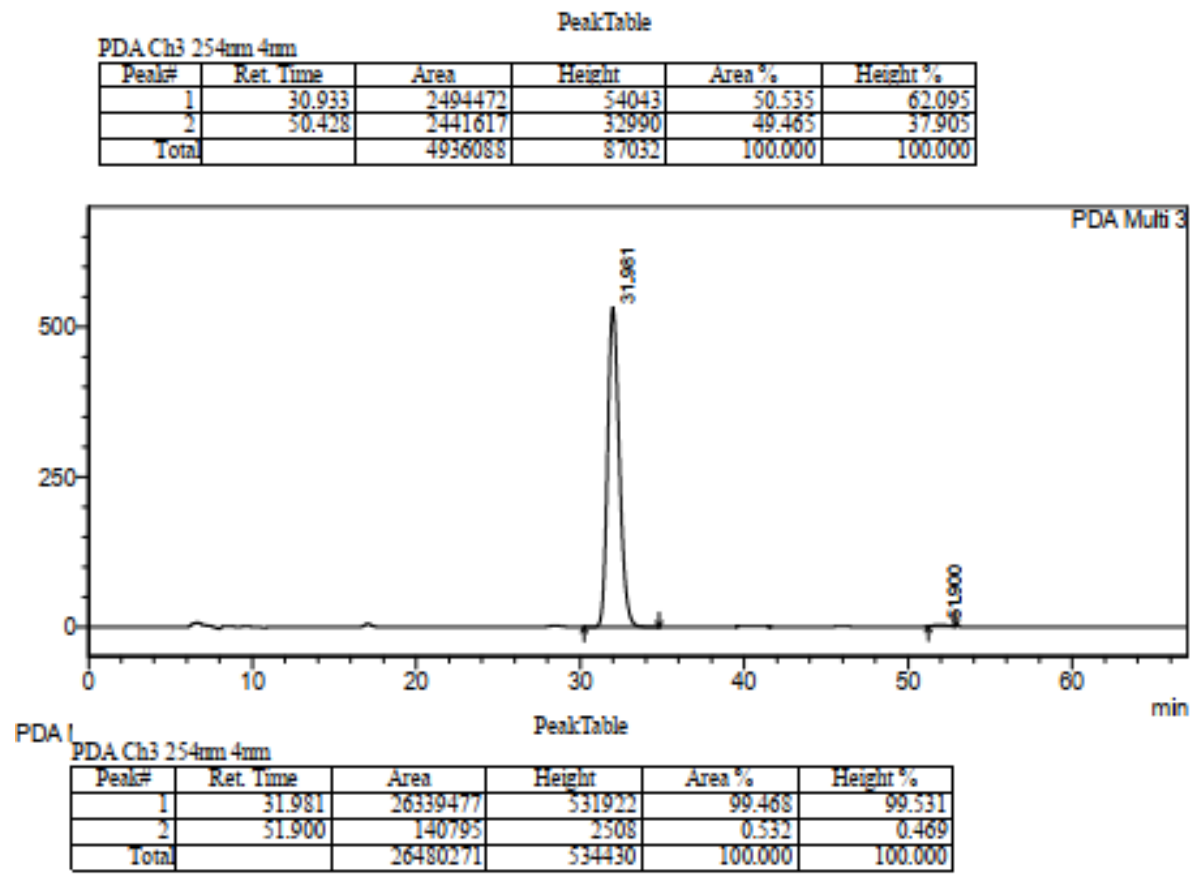

\section{The X-ray crystal data of product $3 v$ and $6 a b$}

CCDC 1952196 (3v) and 1952197 (6ab) contain the supplementary crystallo-graphic data for this paper. These data can be obtained free of charge via www.ccdc.cam.ac.uk/data request/cif<smiles>O=C(N[C@]1(CCS(=O)(=O)F)C(=O)N(Cc2ccccc2)c2ccccc21)C1CC1</smiles>

3v

Identification code

Empirical formula

Formula weight

Temperature/K

Crystal system

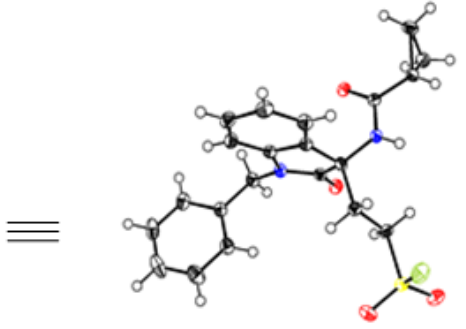

3v CCDC 1952196

$3 \mathrm{v}$

$\mathrm{C}_{21} \mathrm{H}_{21} \mathrm{~N}_{2} \mathrm{O}_{4} \mathrm{SF}$

416.46

100.00(10)

tetragonal 


\begin{tabular}{|c|c|}
\hline Space group & $\mathrm{P} 4_{3}$ \\
\hline $\mathrm{a} / \AA$ & $11.15080(10)$ \\
\hline $\mathrm{b} / \AA ̊$ & $11.15080(10)$ \\
\hline$c / \AA$ & $16.05760(10)$ \\
\hline$\alpha /{ }^{\circ}$ & 90 \\
\hline$\beta /{ }^{\circ}$ & 90 \\
\hline$\gamma /{ }^{\circ}$ & 90 \\
\hline Volume/ $\AA^{3}$ & 1996.61(4) \\
\hline Z & 4 \\
\hline$\rho_{\text {calcg }} / \mathrm{cm}^{3}$ & 1.385 \\
\hline$\mu / \mathrm{mm}^{-1}$ & 1.789 \\
\hline $\mathrm{F}(000)$ & 872.0 \\
\hline Crystal size/mm³ & $0.3 \times 0.2 \times 0.1$ \\
\hline Radiation & $\mathrm{CuK} \alpha(\lambda=1.54184)$ \\
\hline $2 \Theta$ range for data collection $/{ }^{\circ}$ & 7.928 to 153.86 \\
\hline Index ranges & $-14 \leq \mathrm{h} \leq 14,-12 \leq \mathrm{k} \leq 13,-19 \leq 1 \leq 19$ \\
\hline Reflections collected & 20194 \\
\hline Independent reflections & $4121\left[\mathrm{R}_{\text {int }}=0.0251, \mathrm{R}_{\text {sigma }}=0.0156\right]$ \\
\hline Data/restraints/parameters & $4121 / 1 / 262$ \\
\hline Goodness-of-fit on $\mathrm{F}^{2}$ & 1.066 \\
\hline Final R indexes $[\mathrm{I}>=2 \sigma(\mathrm{I})]$ & $\mathrm{R}_{1}=0.0256, \mathrm{wR}_{2}=0.0667$ \\
\hline Final R indexes [all data] & $\mathrm{R}_{1}=0.0260, \mathrm{wR}_{2}=0.0680$ \\
\hline Largest diff. peak/hole / e $\AA^{-3}$ & $0.18 /-0.29$ \\
\hline Flack parameter & $0.000(5)$ \\
\hline 6ab & CCDC 1952197 \\
\hline Identification code & $6 a b$ \\
\hline Empirical formula & $\mathrm{C}_{30} \mathrm{H}_{24} \mathrm{~N}_{2} \mathrm{O}_{4} \mathrm{~S}$ \\
\hline Formula weight & 508.57 \\
\hline Temperature/K & $100.00(10)$ \\
\hline Crystal system & orthorhombic \\
\hline Space group & $\mathrm{P} 2{ }_{1} 2_{1} 2_{1}$ \\
\hline $\mathrm{a} / \AA$ & 7.92191(4) \\
\hline $\mathrm{b} / \AA$ & $12.56234(7)$ \\
\hline c/Å & 25.20069(13) \\
\hline
\end{tabular}




\begin{tabular}{|c|c|}
\hline$\alpha /{ }^{\circ}$ & 90 \\
\hline$\beta /{ }^{\circ}$ & 90 \\
\hline$\gamma /{ }^{\circ}$ & 90 \\
\hline Volume $/ \AA^{3}$ & 2507.92(2) \\
\hline $\mathrm{Z}$ & 4 \\
\hline$\rho_{\text {calc }} \mathrm{g} / \mathrm{cm}^{3}$ & 1.347 \\
\hline$\mu / \mathrm{mm}^{-1}$ & 1.474 \\
\hline $\mathrm{F}(000)$ & 1064.0 \\
\hline Crystal size/mm³ & $0.2 \times 0.1 \times 0.1$ \\
\hline Radiation & $\operatorname{CuK} \alpha(\lambda=1.54184)$ \\
\hline $2 \Theta$ range for data collection ${ }^{\circ}$ & 7.016 to 154.038 \\
\hline Index ranges & $-7 \leq \mathrm{h} \leq 9,-15 \leq \mathrm{k} \leq 15,-30 \leq 1 \leq 31$ \\
\hline Reflections collected & 25706 \\
\hline Independent reflections & $5122\left[\mathrm{R}_{\text {int }}=0.0306, \mathrm{R}_{\text {sigma }}=0.0197\right]$ \\
\hline Data/restraints/parameters & $5122 / 0 / 335$ \\
\hline Goodness-of-fit on $\mathrm{F}^{2}$ & 1.047 \\
\hline Final R indexes $[\mathrm{I}>=2 \sigma(\mathrm{I})]$ & $\mathrm{R}_{1}=0.0246, \mathrm{wR}_{2}=0.0625$ \\
\hline Final R indexes [all data] & $\mathrm{R}_{1}=0.0259, \mathrm{wR}_{2}=0.0633$ \\
\hline Largest diff. peak/hole / e $\AA^{-3}$ & $0.20 /-0.26$ \\
\hline Flack parameter & $0.011(4)$ \\
\hline
\end{tabular}

\section{References}

1. Pei, C. K.; Jiang, Y.; Shi, M. Eur. J. Org. Chem. 2012, 2012, 4206-4216.

2. Z Zheng, Q.; Dong, J.; Sharpless, K. B. J. Org. Chem. 2016, 81, 11360-11362.

3. Zha, G. F.; Zheng, Q.; Leng, J.; Wu, P.; Qin, H. L.; Sharpless, K. B. Angew. Chem., Int. Ed. 2017, 56, 4849-4852.

4. Zha, G. F.; Bare, G. A. L.; Leng, J.; Shang, Z. P.; Luo, Z.; Qin, H. L. Adv. Synth. Catal. 2017, 359, 3237-3242.

5. Wang, J.; Li, H.; Zu, L.; Jiang, W.; Xie, H.; Duan, W.; Wang, W. J. Am. Chem. Soc. 2006, 128, 12652-3.

6. $\quad$ Wang, Y. Q.; Song, J.; Hong, R.; Li, H.; Deng, L. J. Am. Chem. Soc. 2006, 128, 8156-7.

7. Badiola, E.; Fiser, B.; Gomez Bengoa, E.; Mielgo, A.; Olaizola, I.; Urruzuno, I.; Garcia, J. M.; Odriozola, J. M.; Razkin, J.; Oiarbide, M.; Palomo, C. J. Am. Chem. Soc. 2014, 136, 17869-81.

8. Porta, R.; Benaglia, M.; Coccia, F.; Cozzi, F.; Puglisi, A. Adv. Synth. Catal. 2015, 357, 377-383.

9. Rout, S.; Joshi, H.; Singh, V. K. Org. Lett. 2018, 20, 2199-2203. 


\section{NMR spectra}

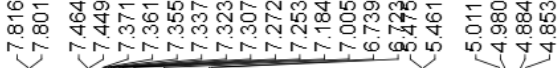
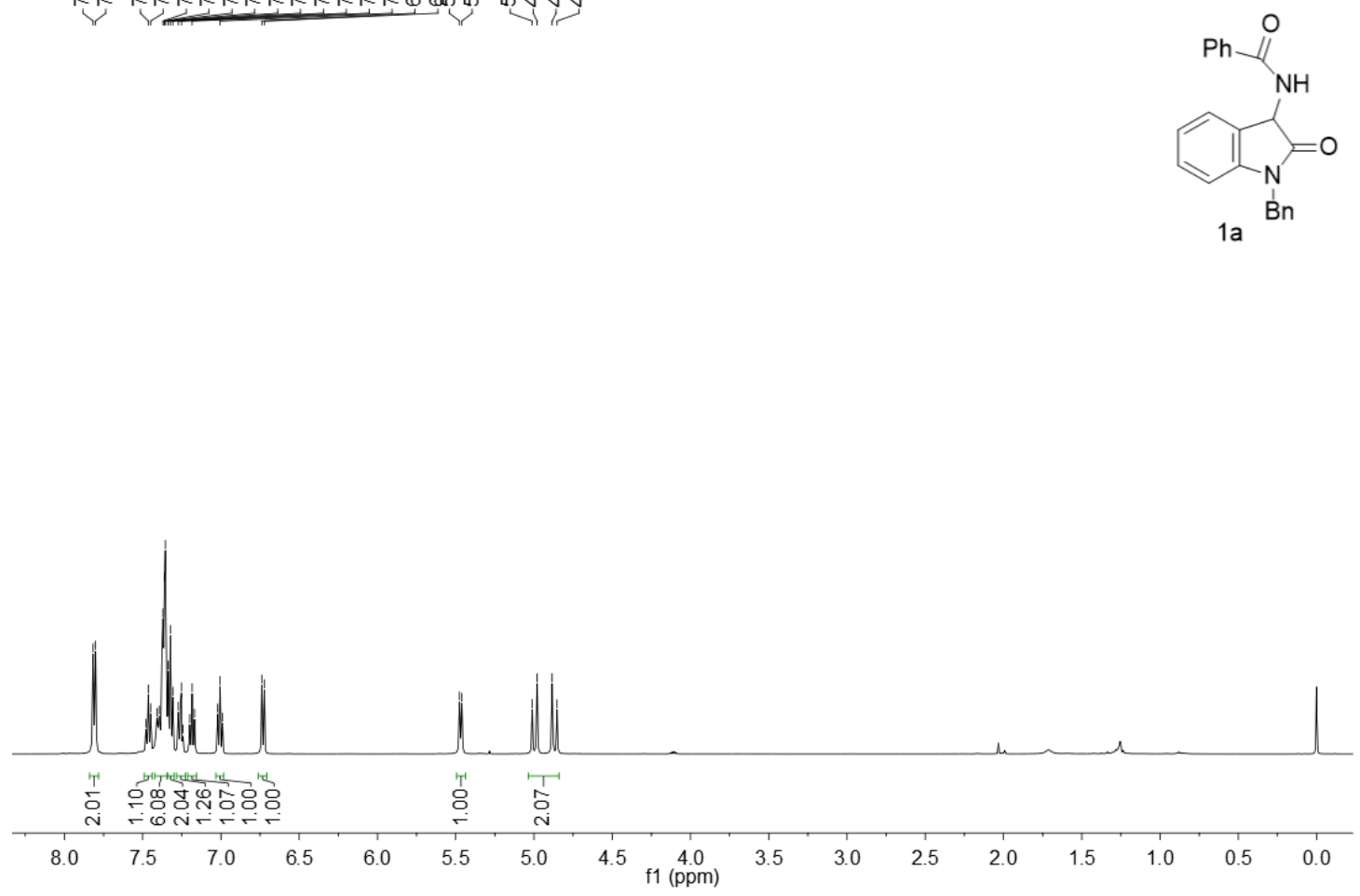

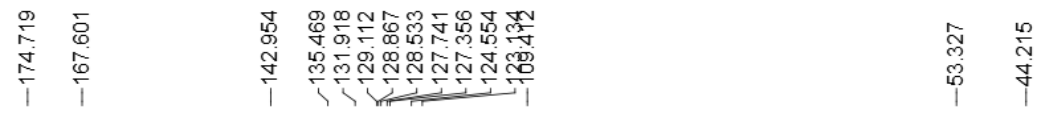
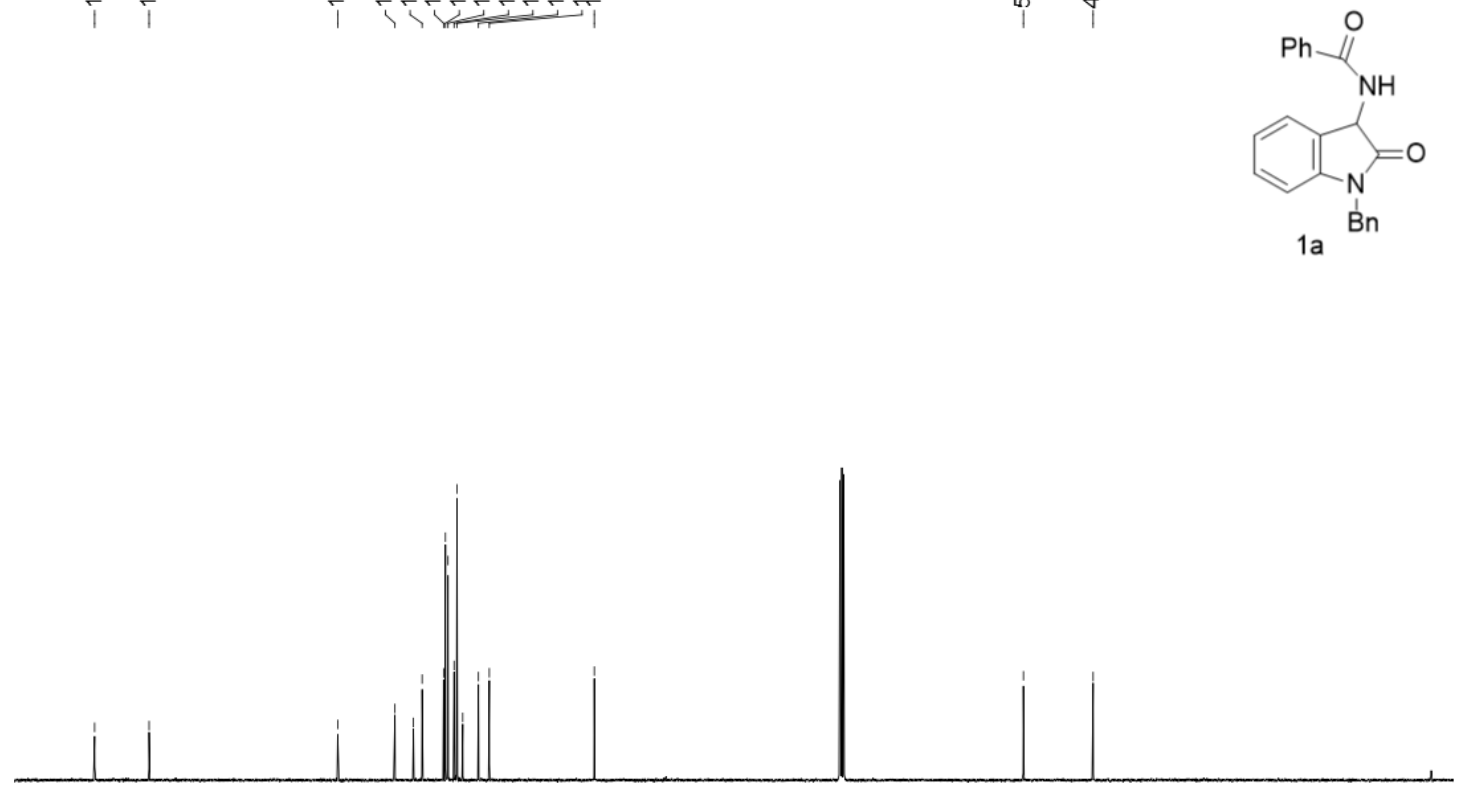

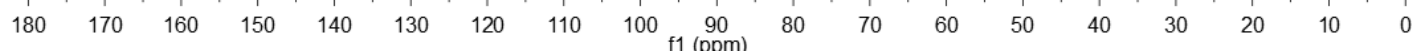

${ }^{1} \mathrm{H}$ and ${ }^{13} \mathrm{C}$ NMR spectra of $\mathbf{1 a}$ 

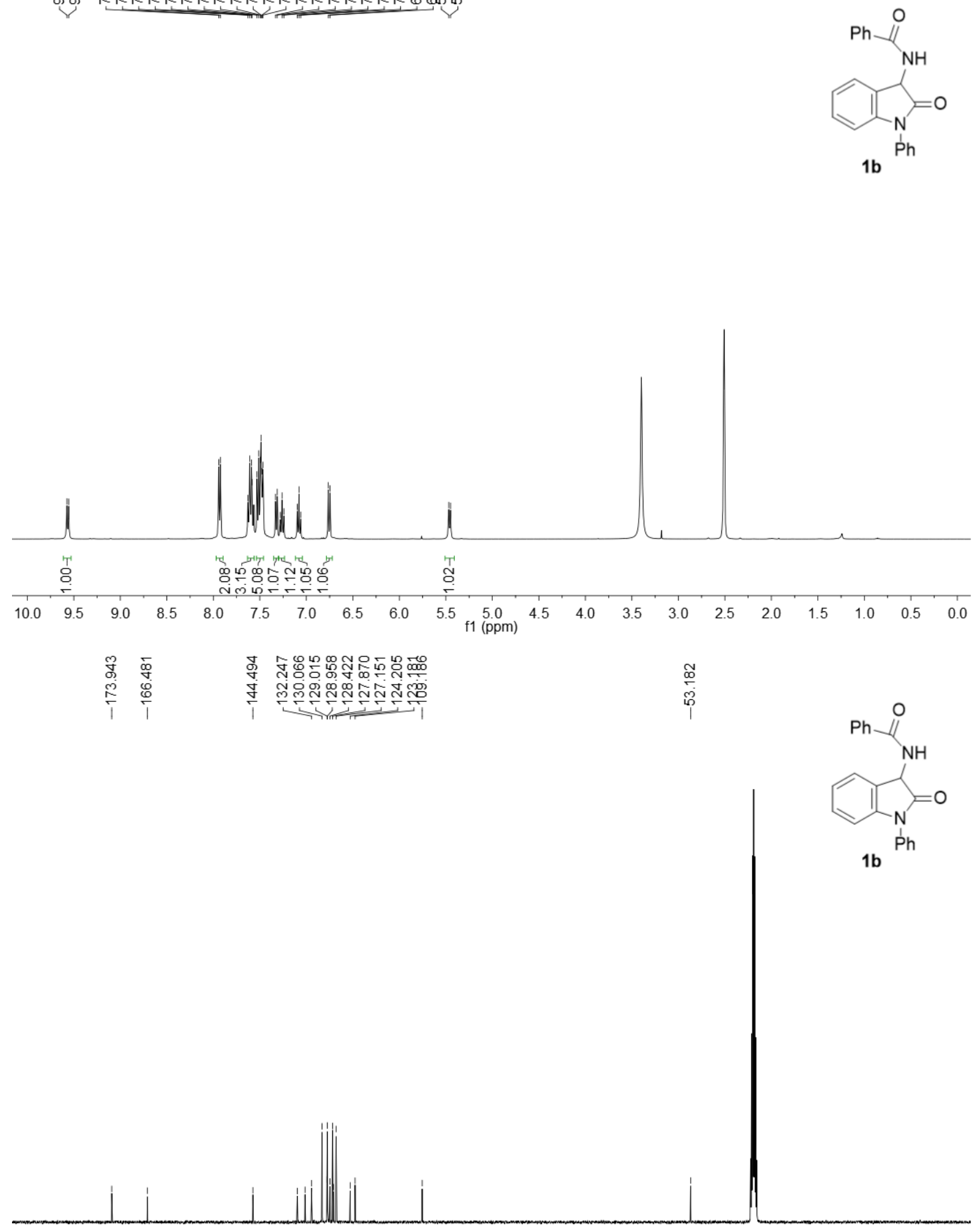

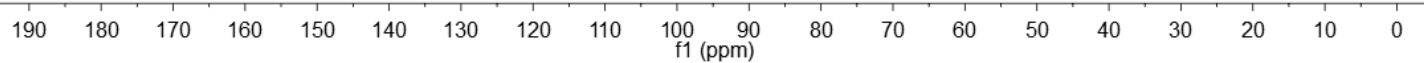

${ }^{1} \mathrm{H}$ and ${ }^{13} \mathrm{C}$ NMR spectra of $\mathbf{1 b}$ 

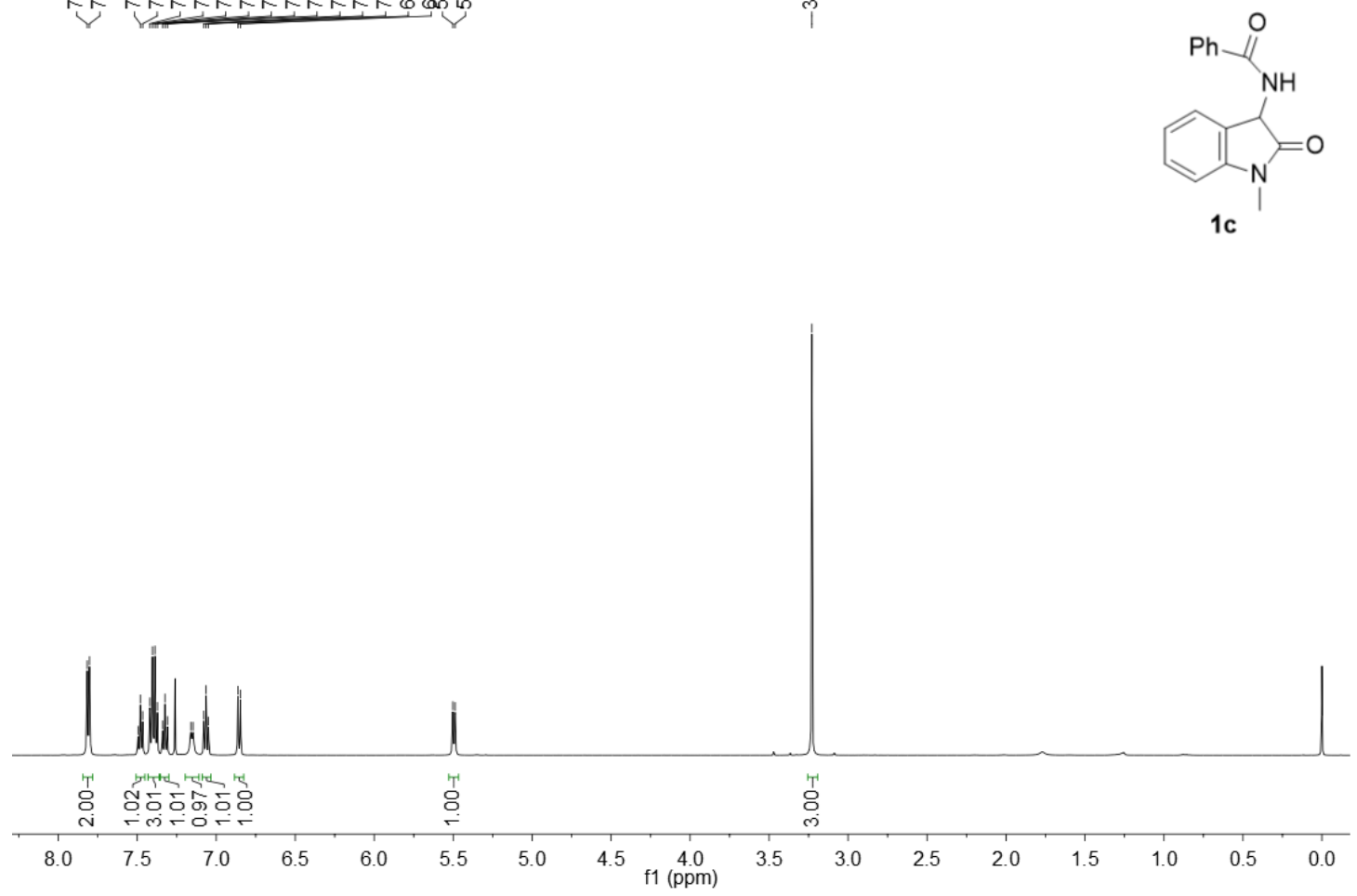

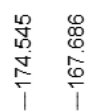

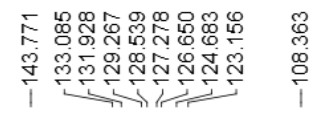

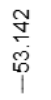

$\underset{\substack{0 \\ 0 \\ 0}}{1}$
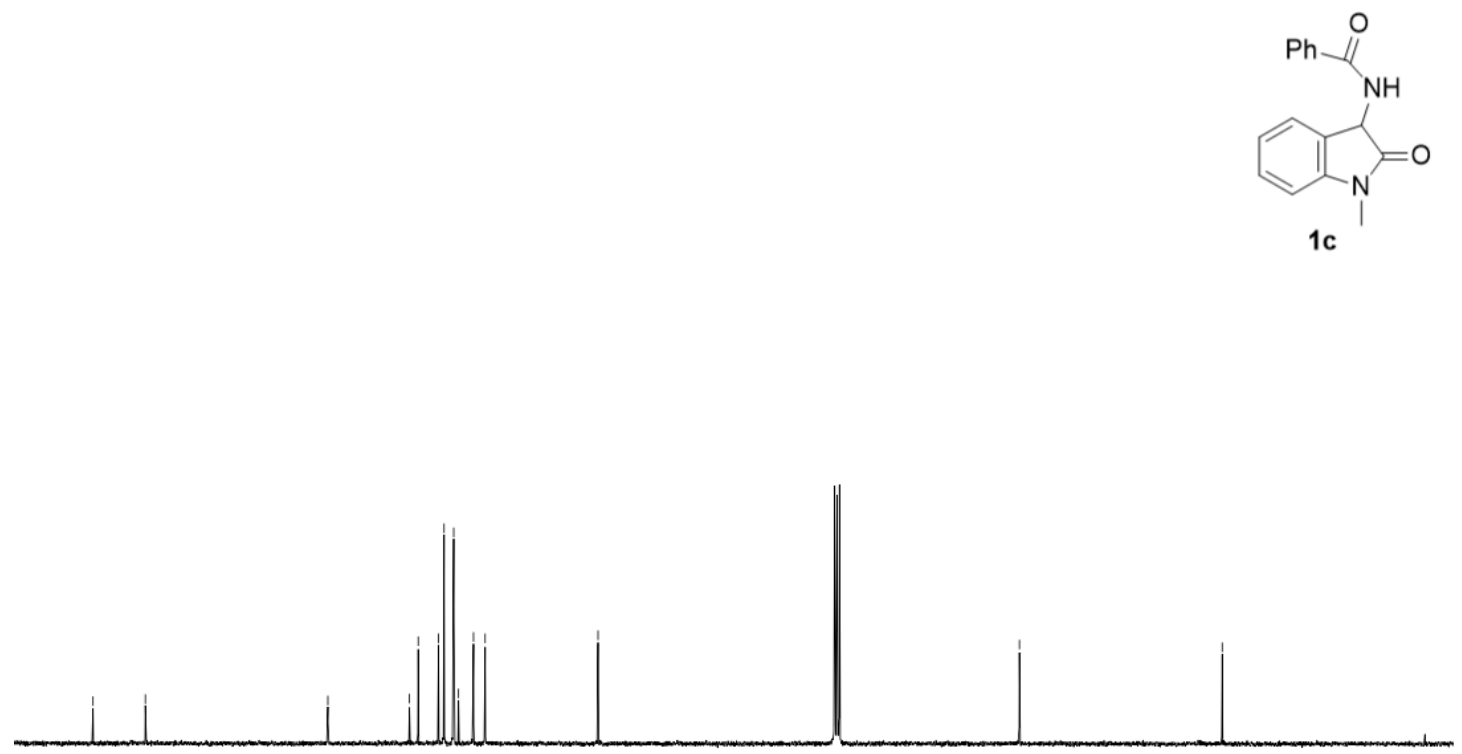

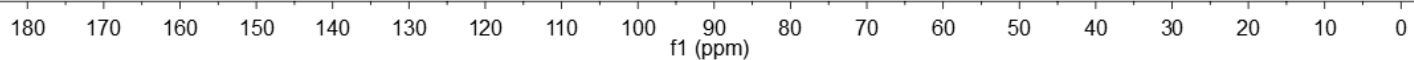

${ }^{1} \mathrm{H}$ and ${ }^{13} \mathrm{C}$ NMR spectra of $\mathbf{1 c}$ 


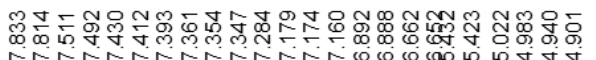

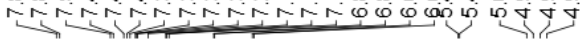

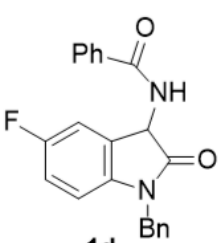

1d $^{\mathrm{Bn}}$
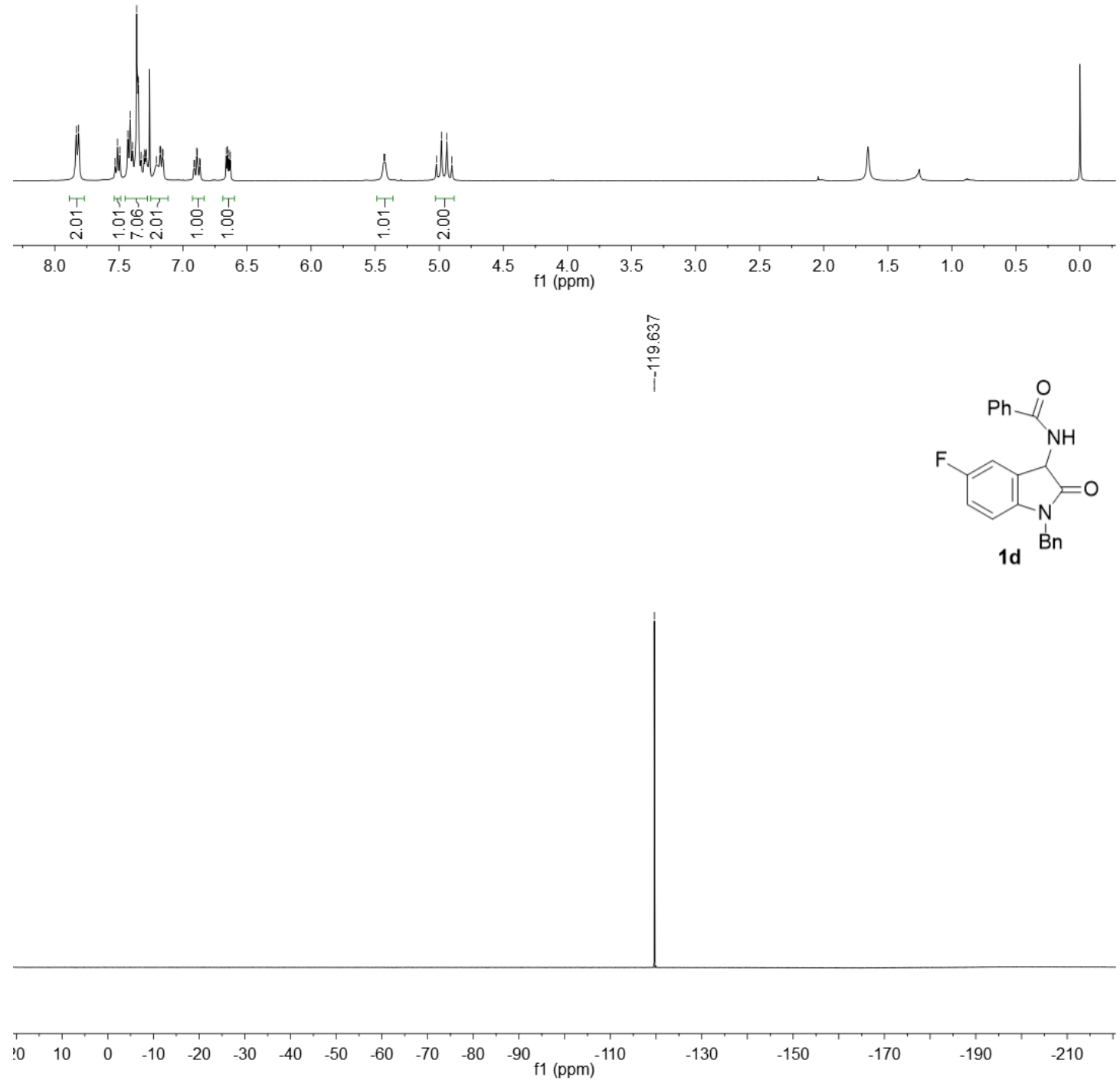

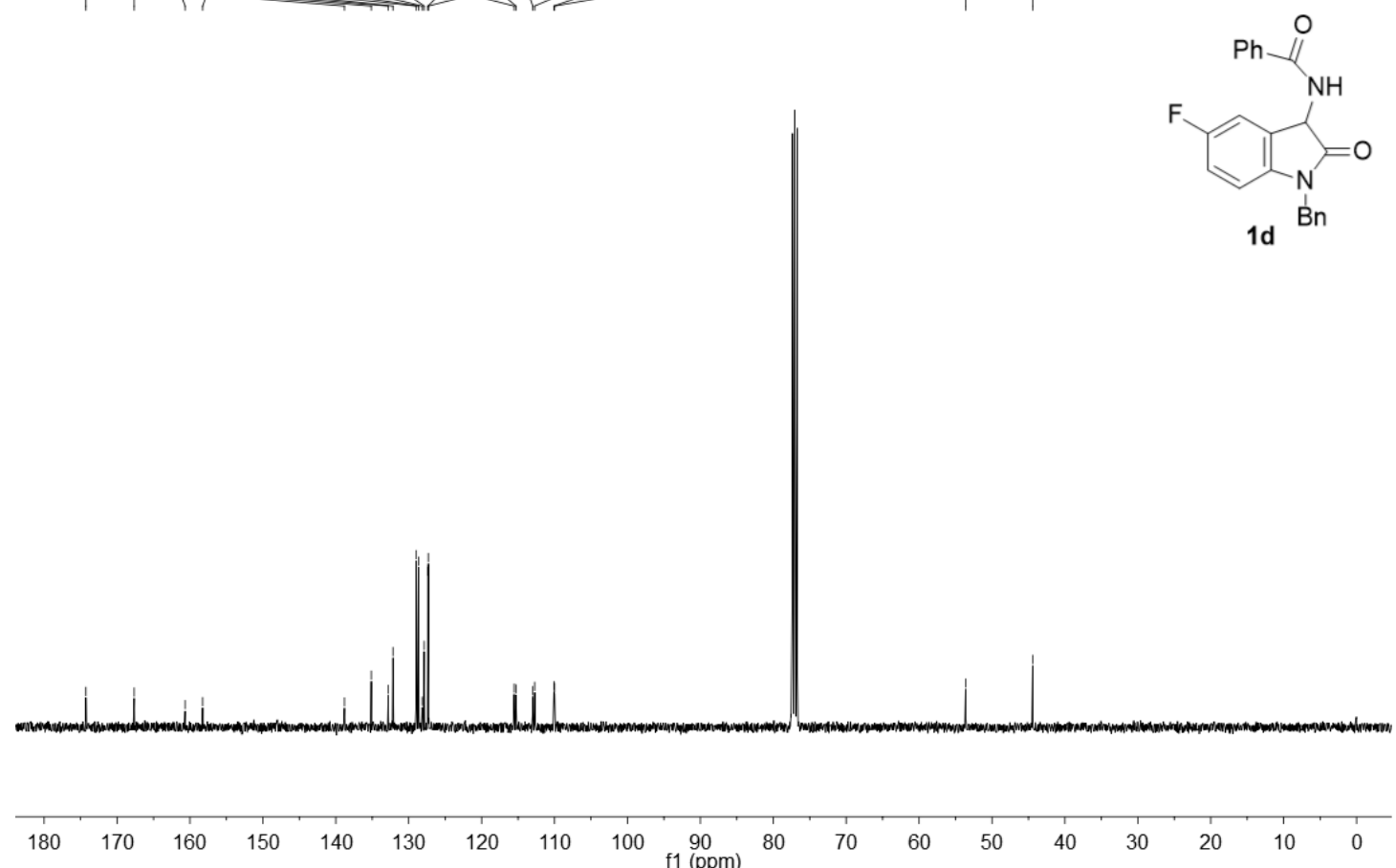

${ }^{1} \mathrm{H},{ }^{19} \mathrm{~F}$ and ${ }^{13} \mathrm{C}$ NMR spectra of $\mathbf{1 d}$

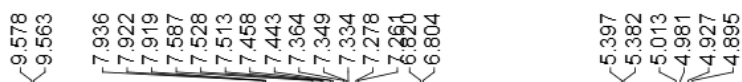
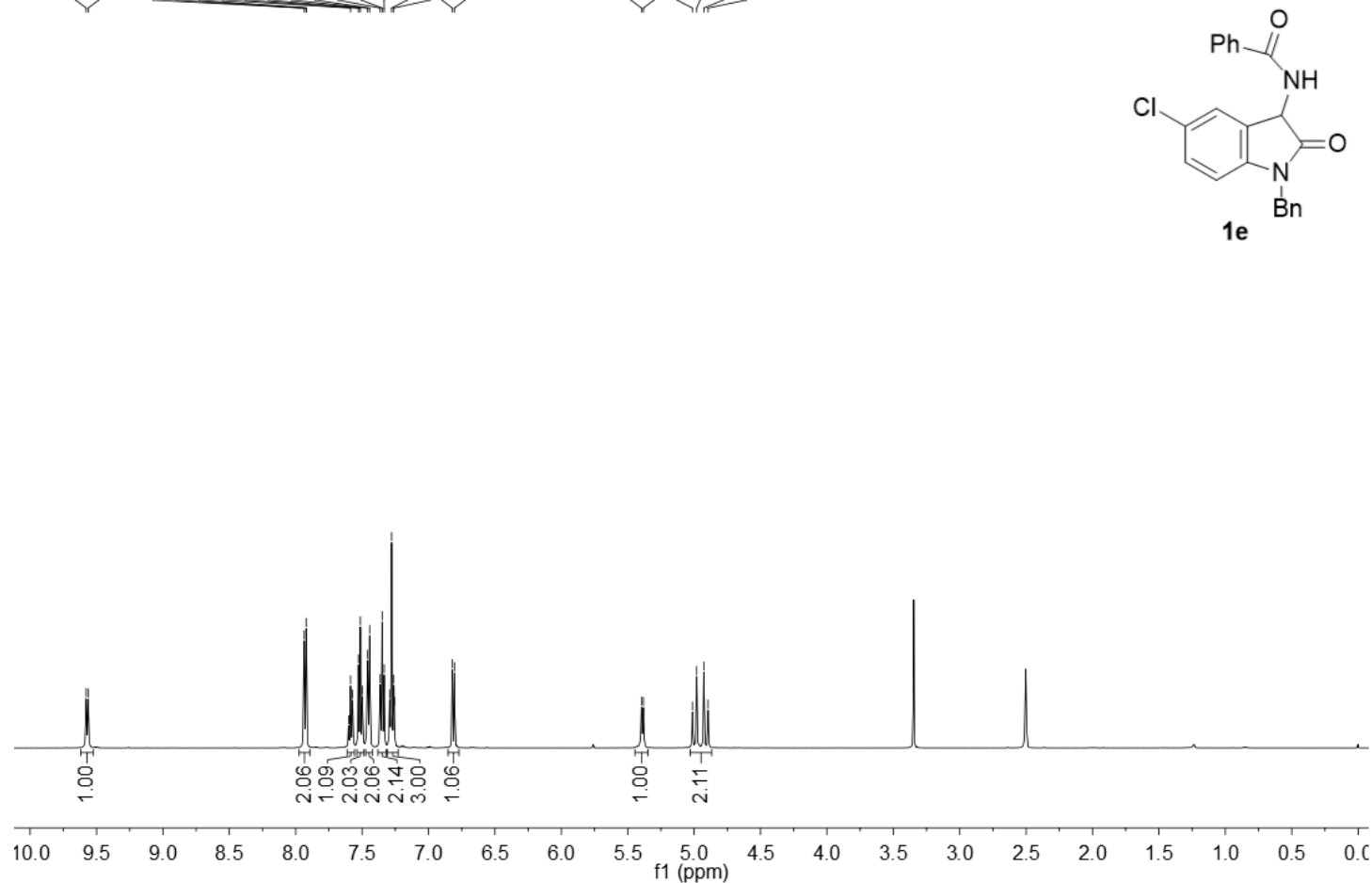


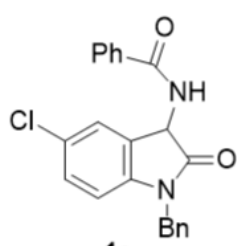

$1 \mathrm{e}$

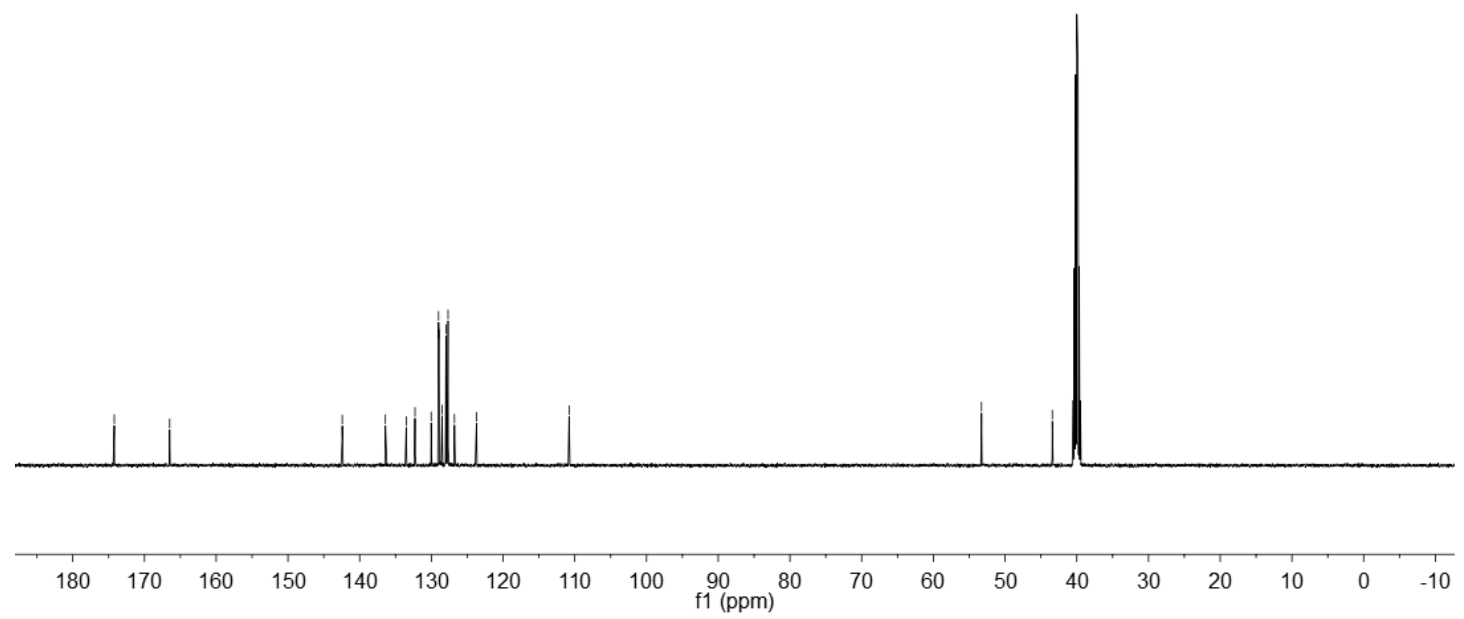

${ }^{1} \mathrm{H}$ and ${ }^{13} \mathrm{C}$ NMR spectra of $\mathbf{1 e}$

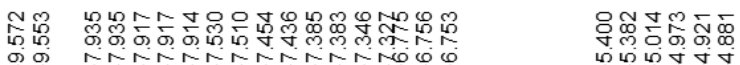

बin
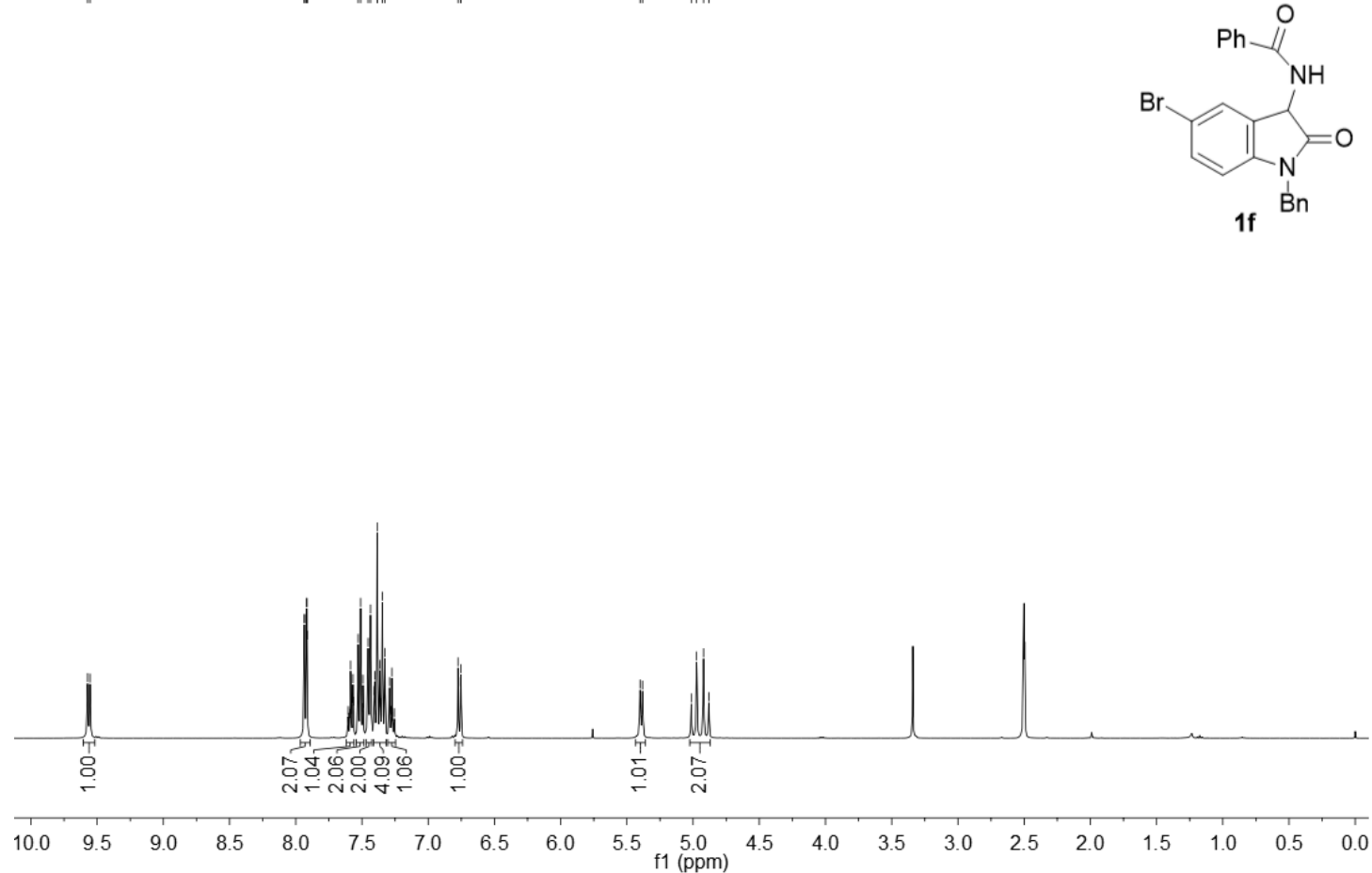


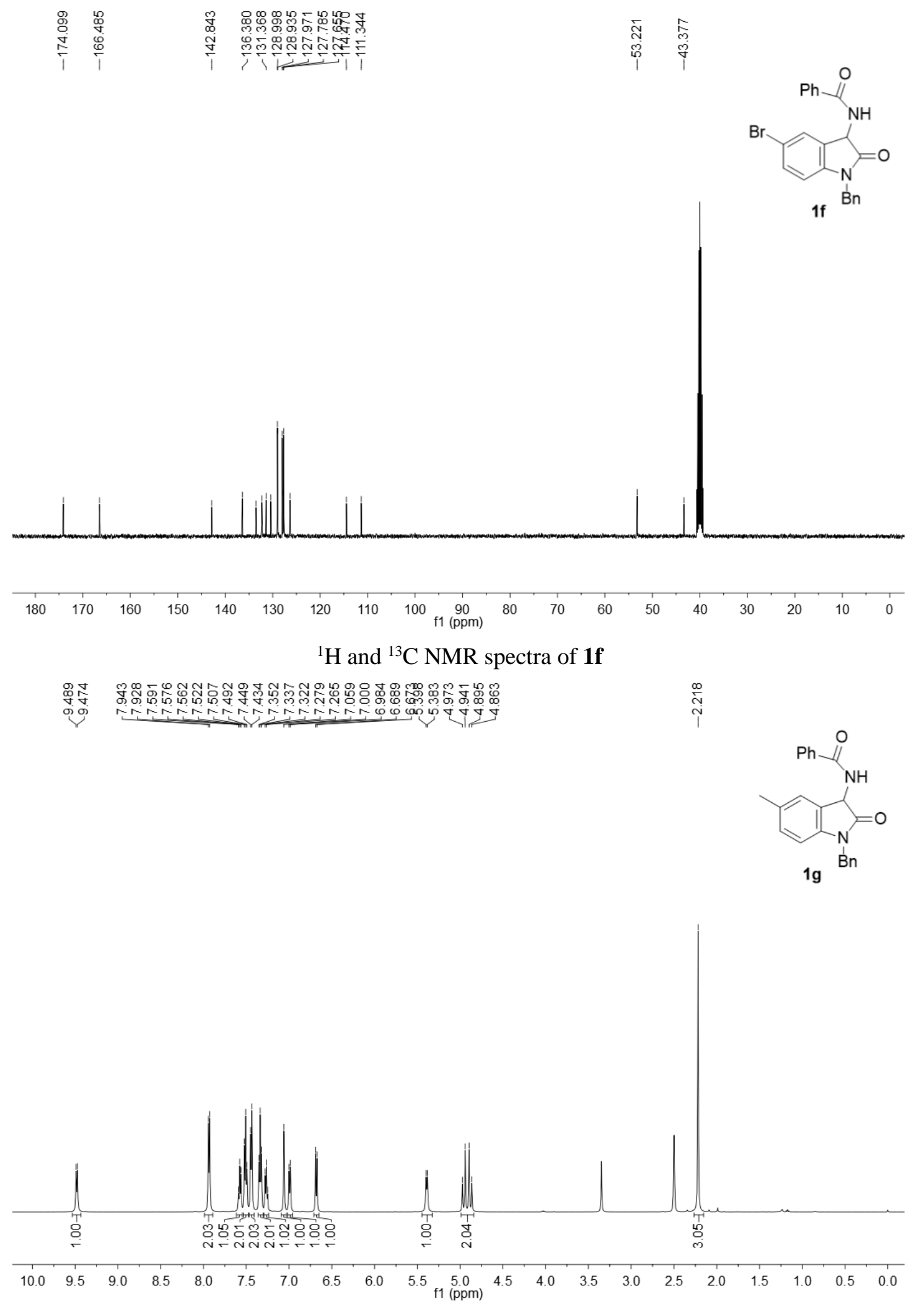




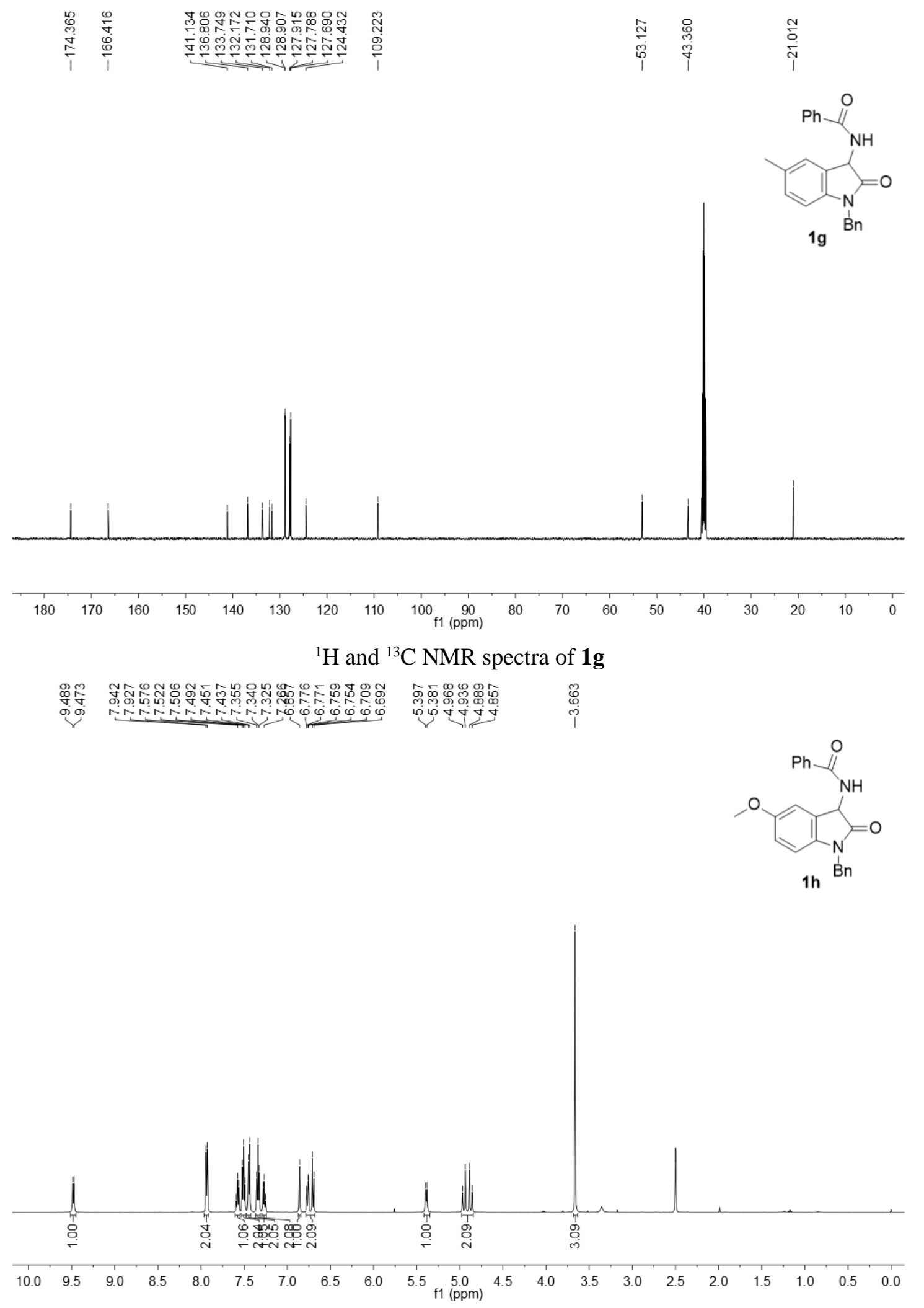




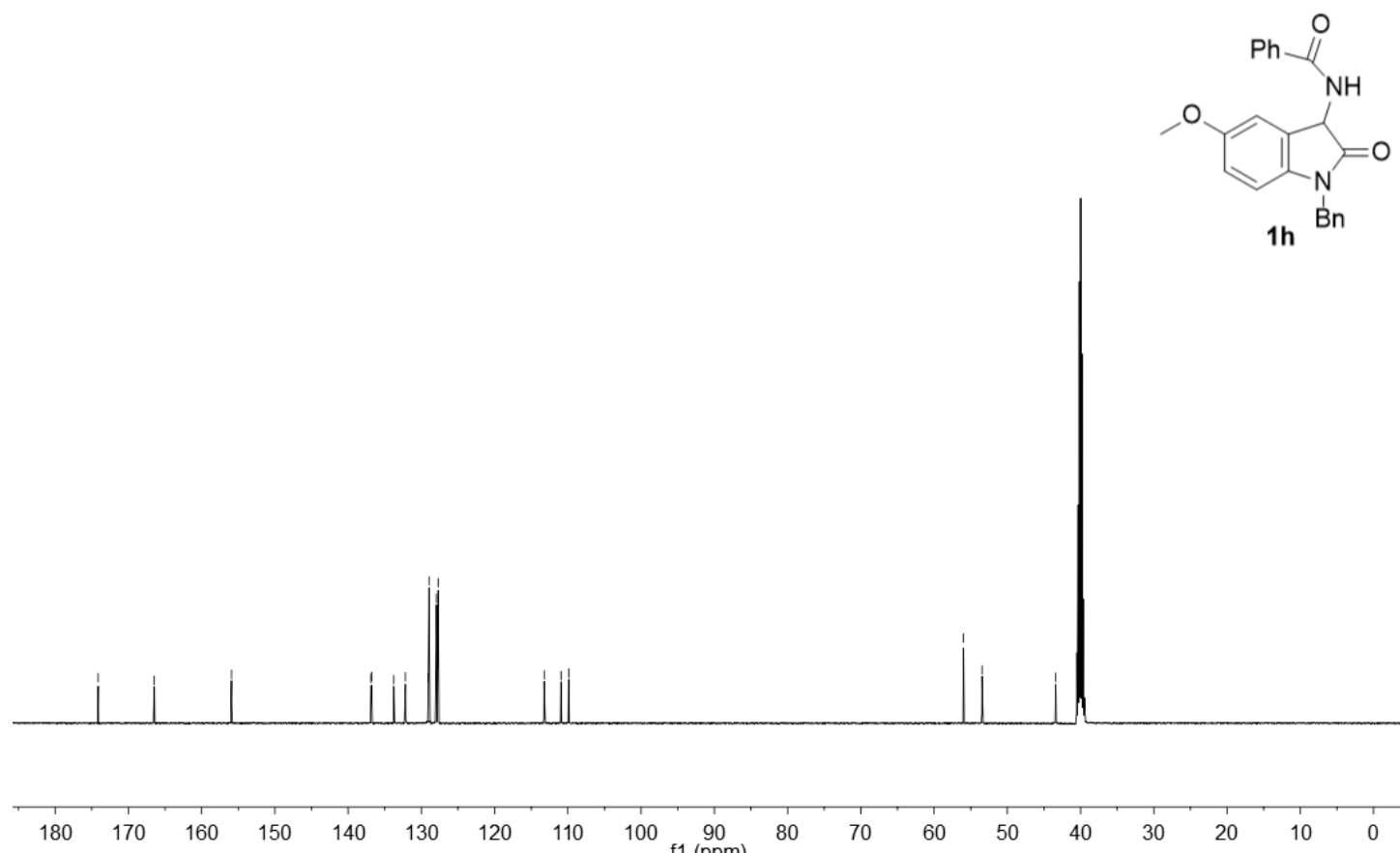

${ }^{1} \mathrm{H}$ and ${ }^{13} \mathrm{C}$ NMR spectra of $\mathbf{1 h}$

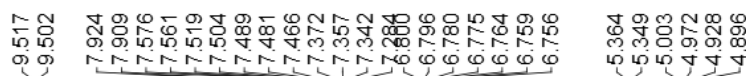
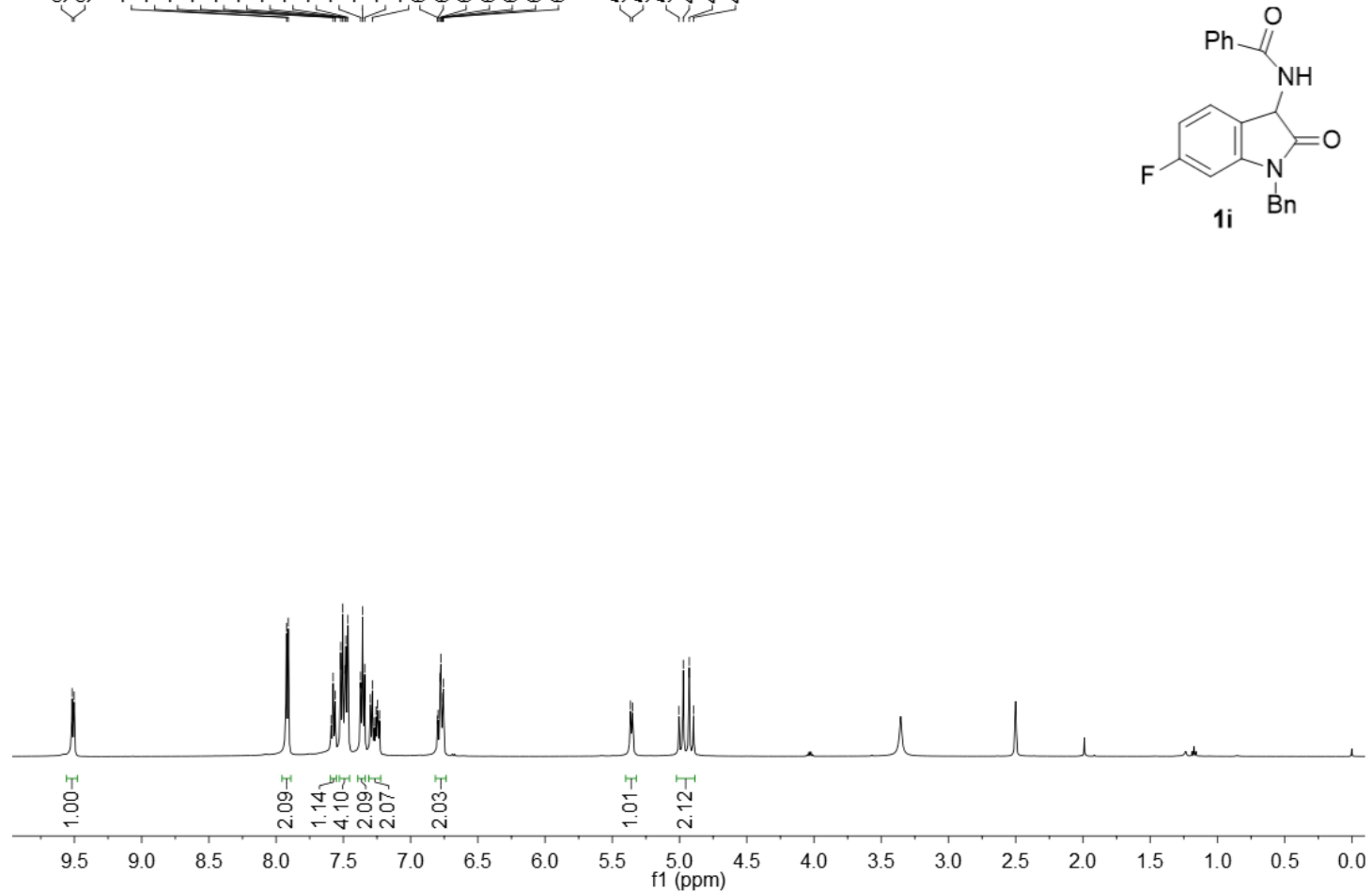
$\underset{\substack{\circ \\ \stackrel{\circ}{\circ}}}{\stackrel{i}{i}}$
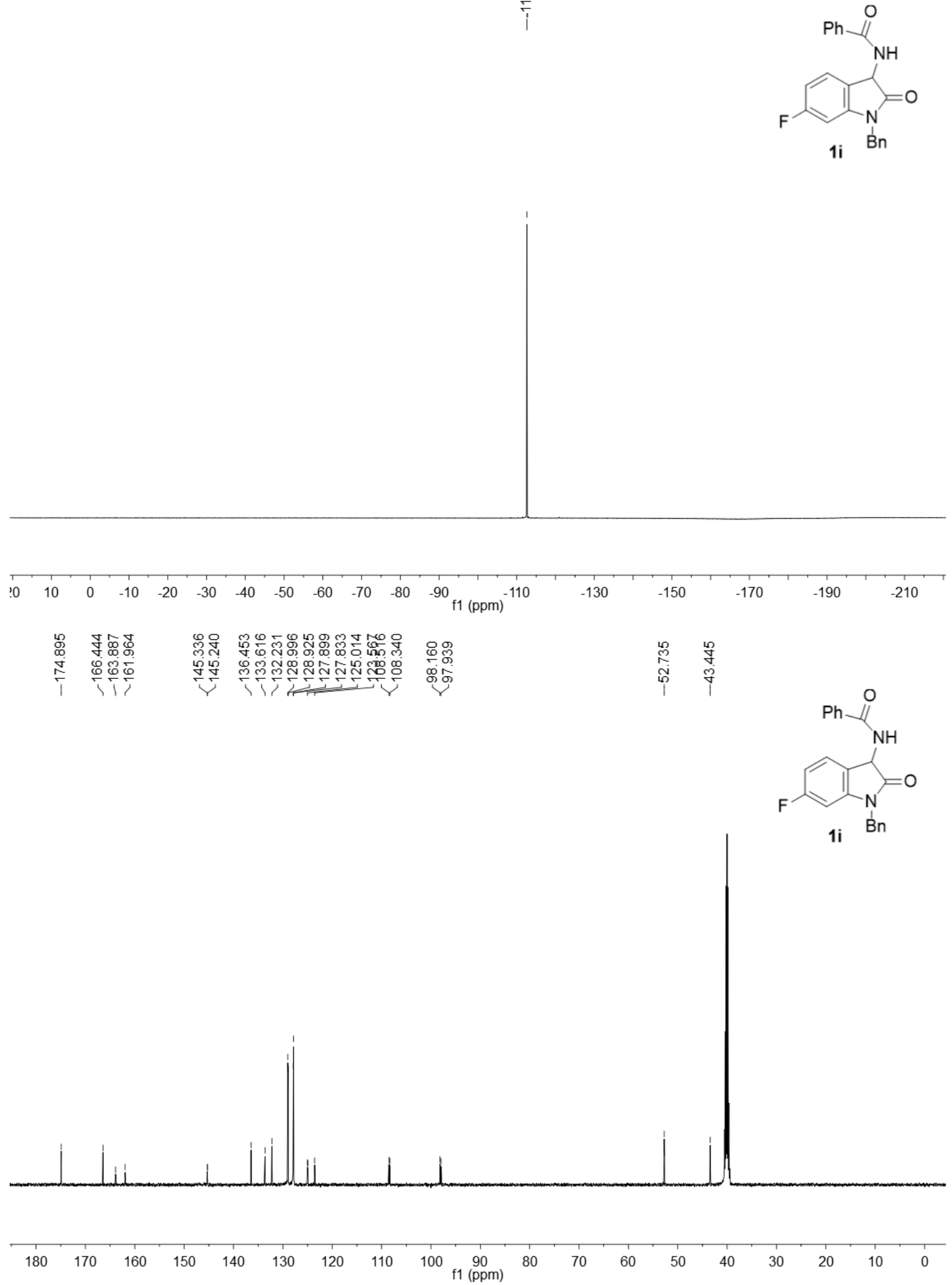

${ }^{1} \mathrm{H},{ }^{19} \mathrm{~F}$ and ${ }^{13} \mathrm{C}$ NMR spectra of $\mathbf{1 i}$ 


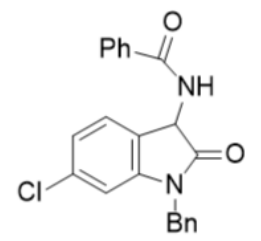

1j

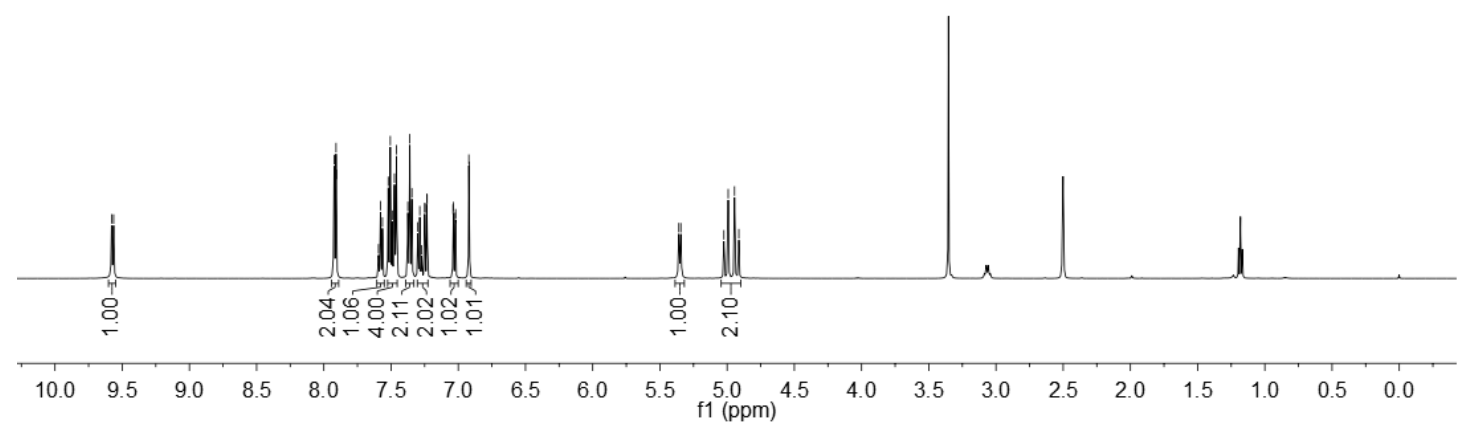

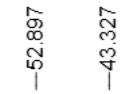

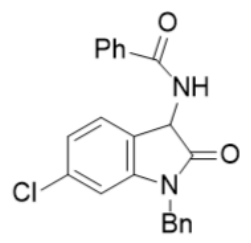

1j

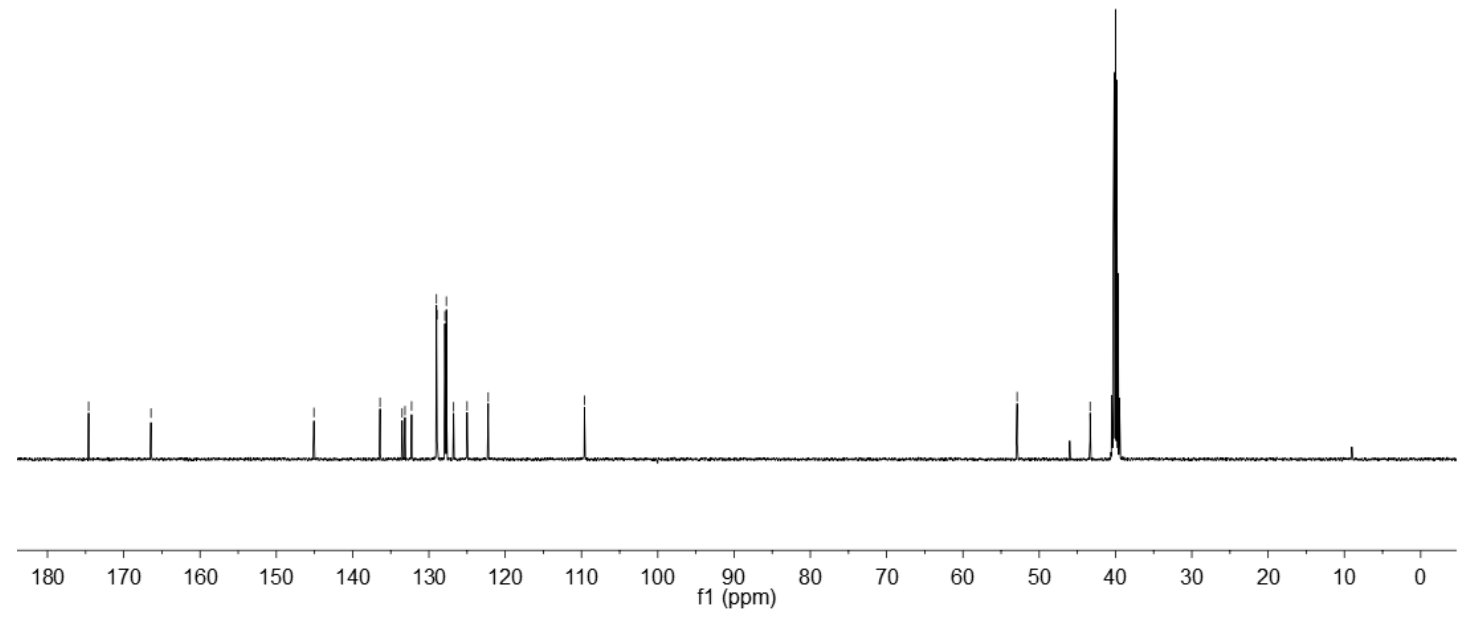

${ }^{1} \mathrm{H}$ and ${ }^{13} \mathrm{C}$ NMR spectra of $\mathbf{1} \mathbf{j}$ 

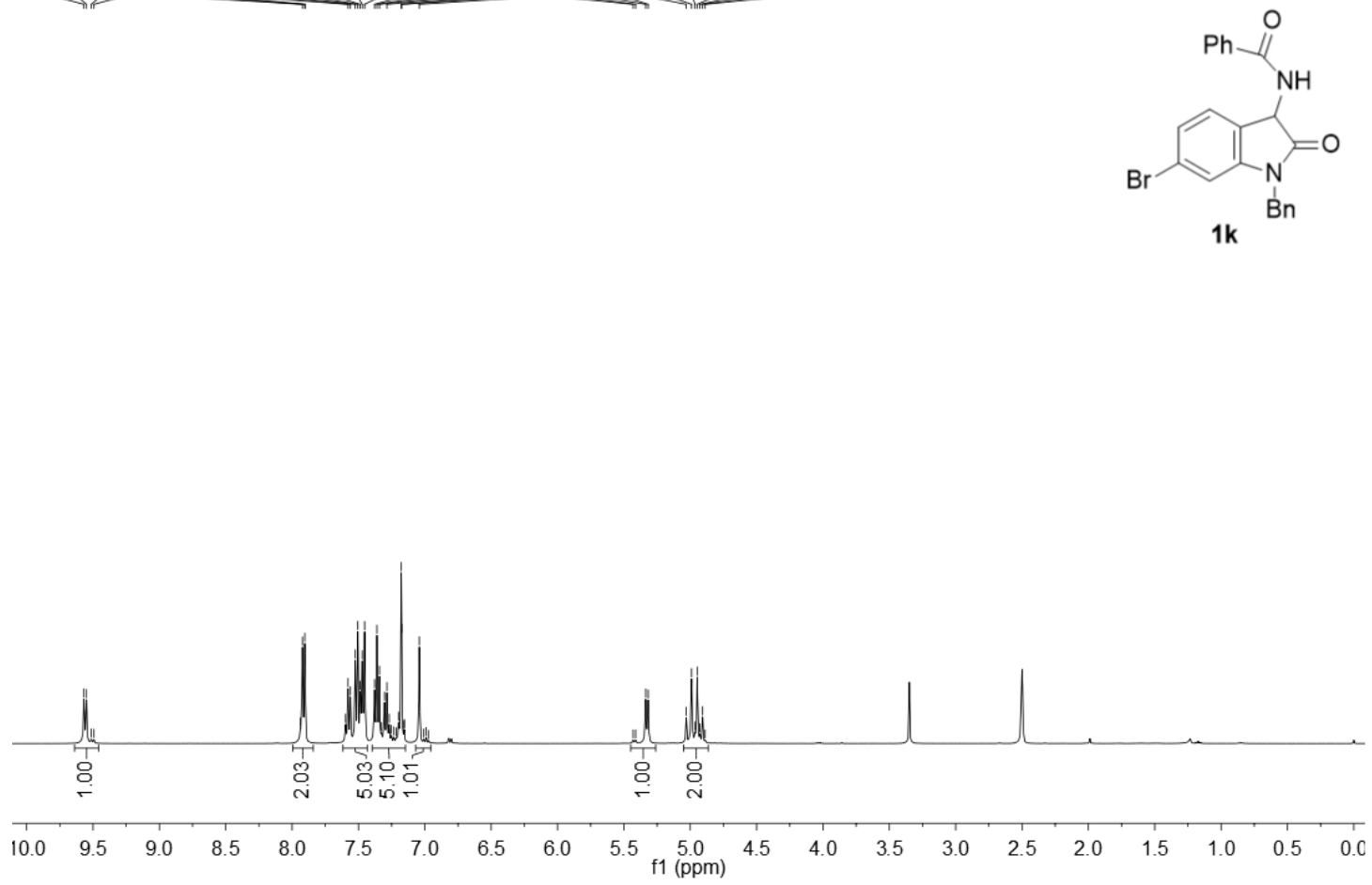

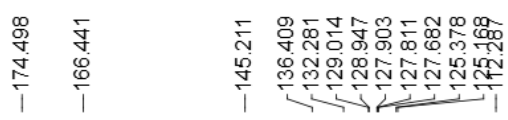

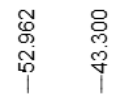
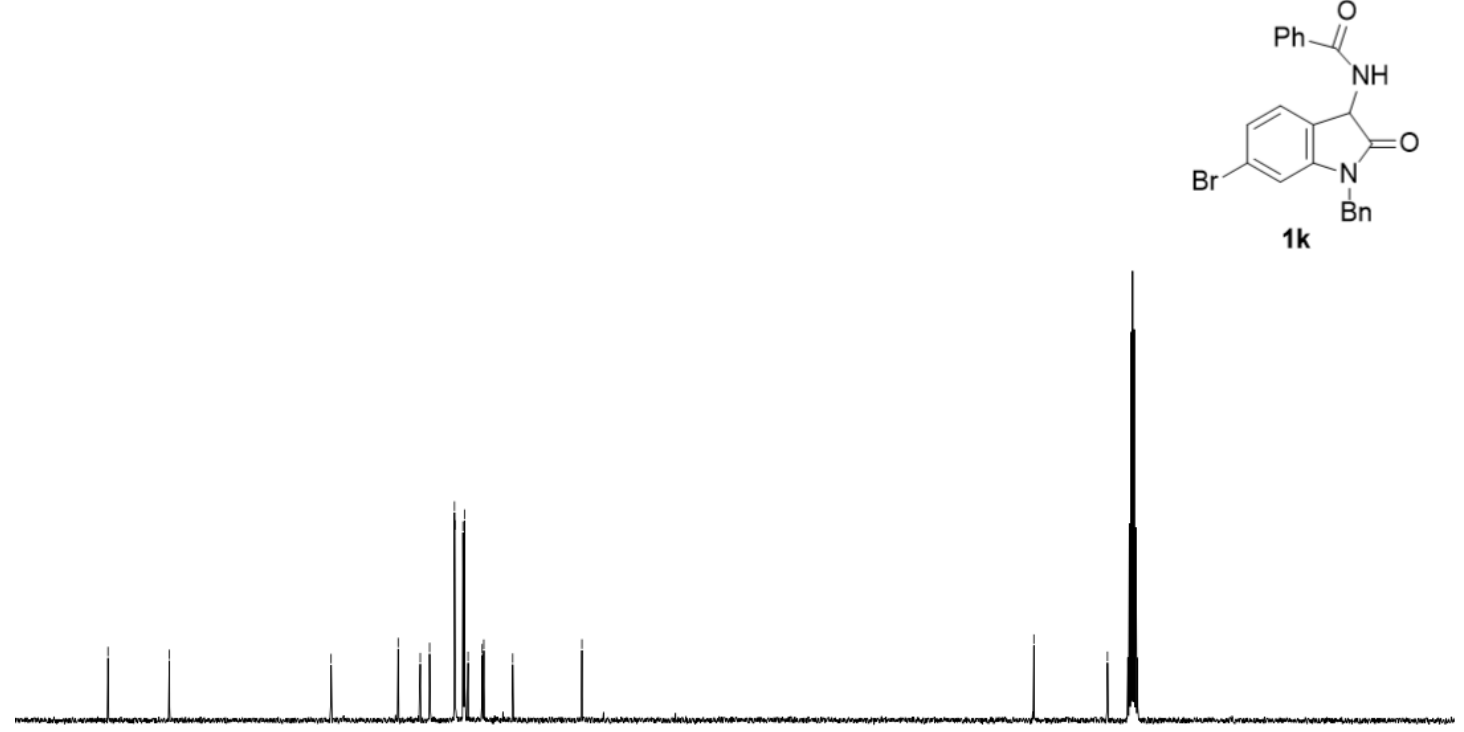

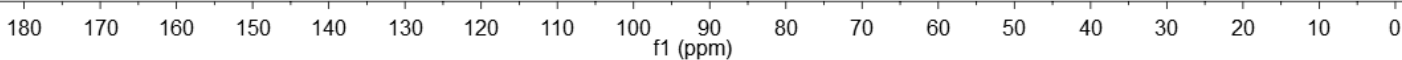

${ }^{1} \mathrm{H}$ and ${ }^{13} \mathrm{C}$ NMR spectra of $\mathbf{1 k}$ 


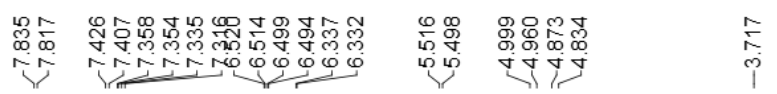
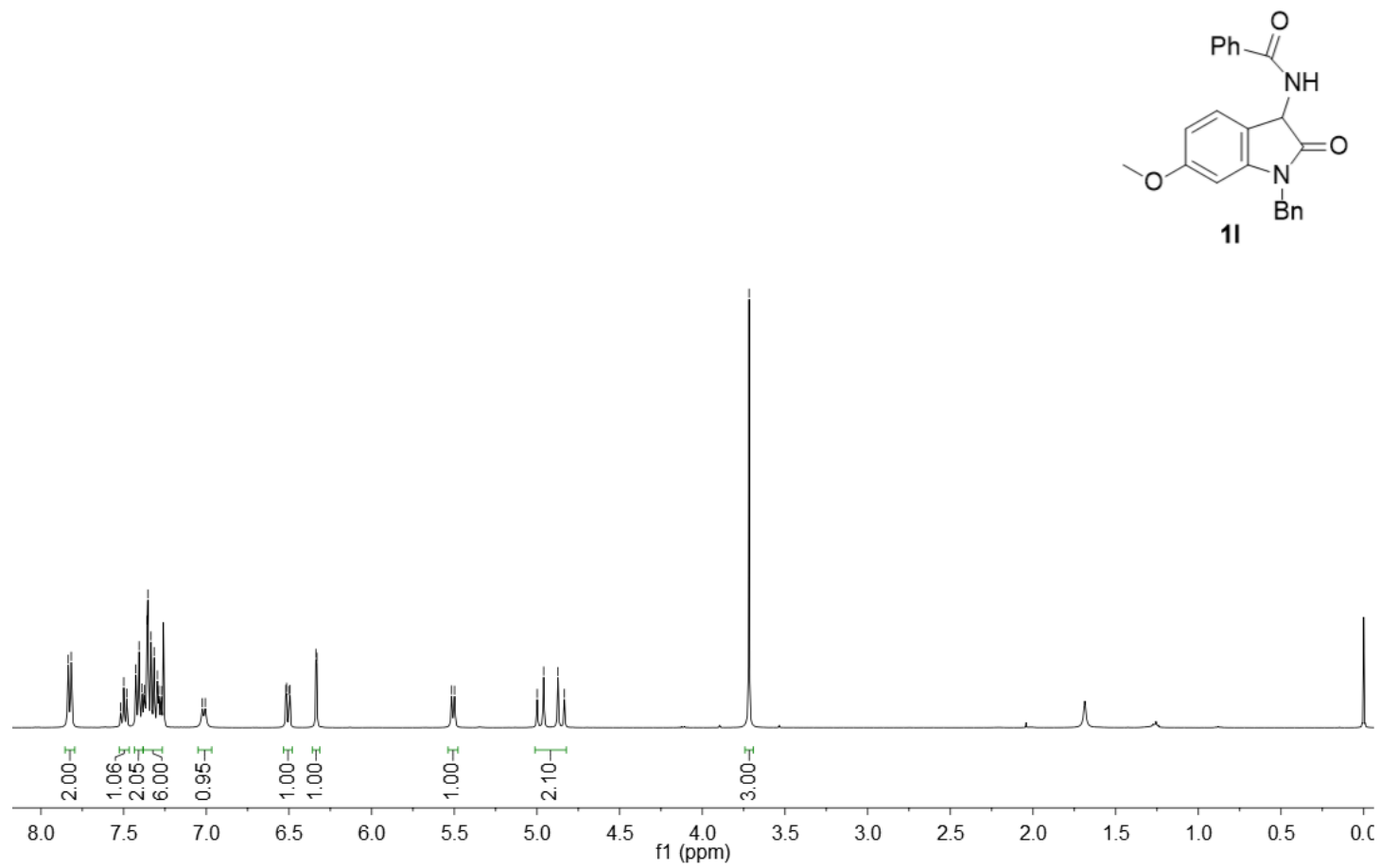

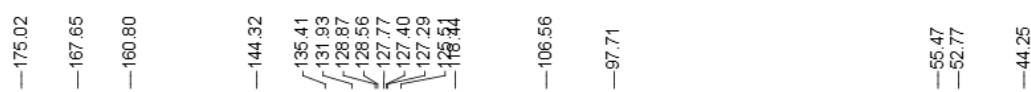
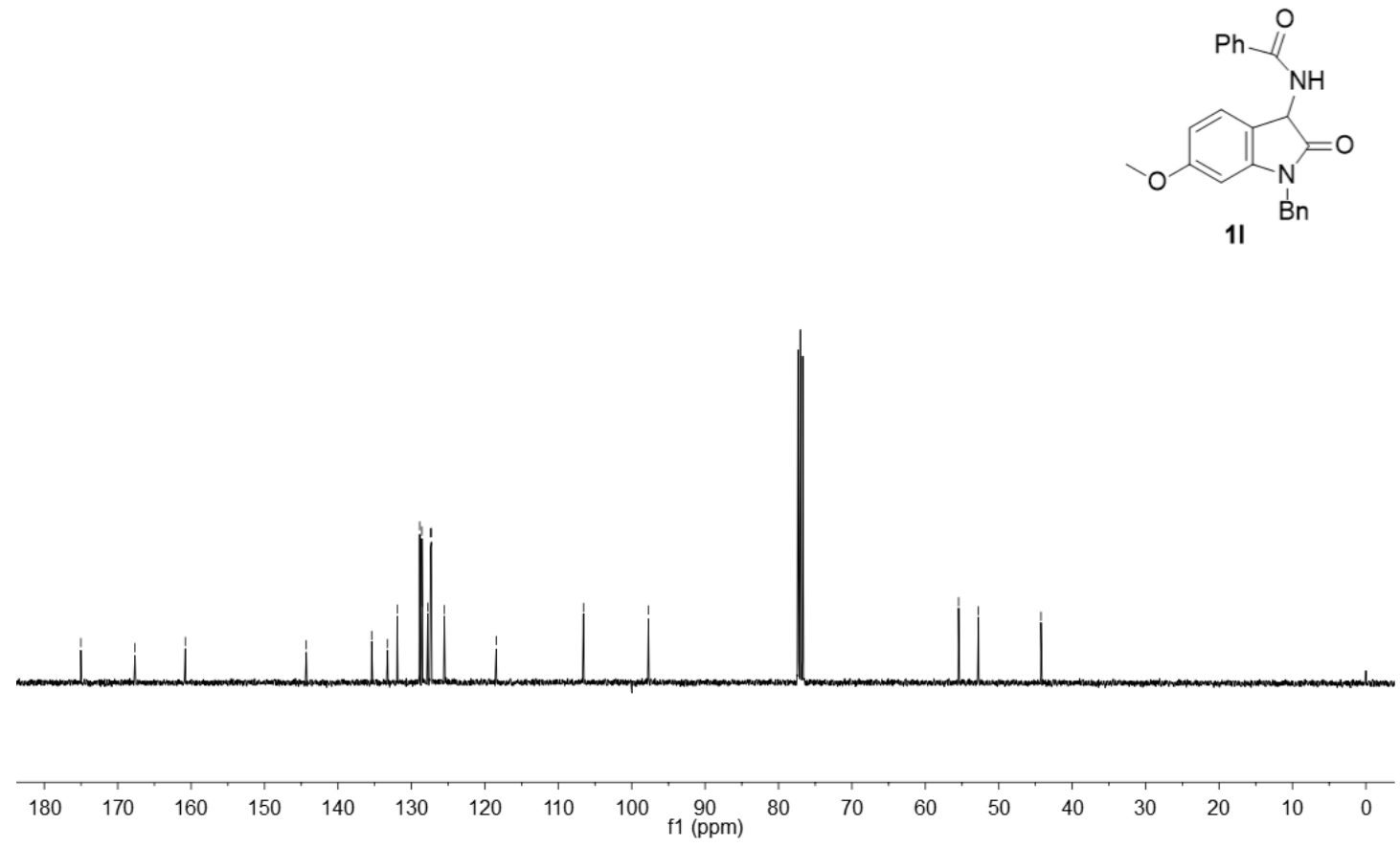

${ }^{1} \mathrm{H}$ and ${ }^{13} \mathrm{C}$ NMR spectra of $\mathbf{1 l}$ 


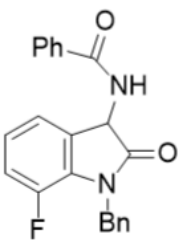

$1 \mathrm{~m}$
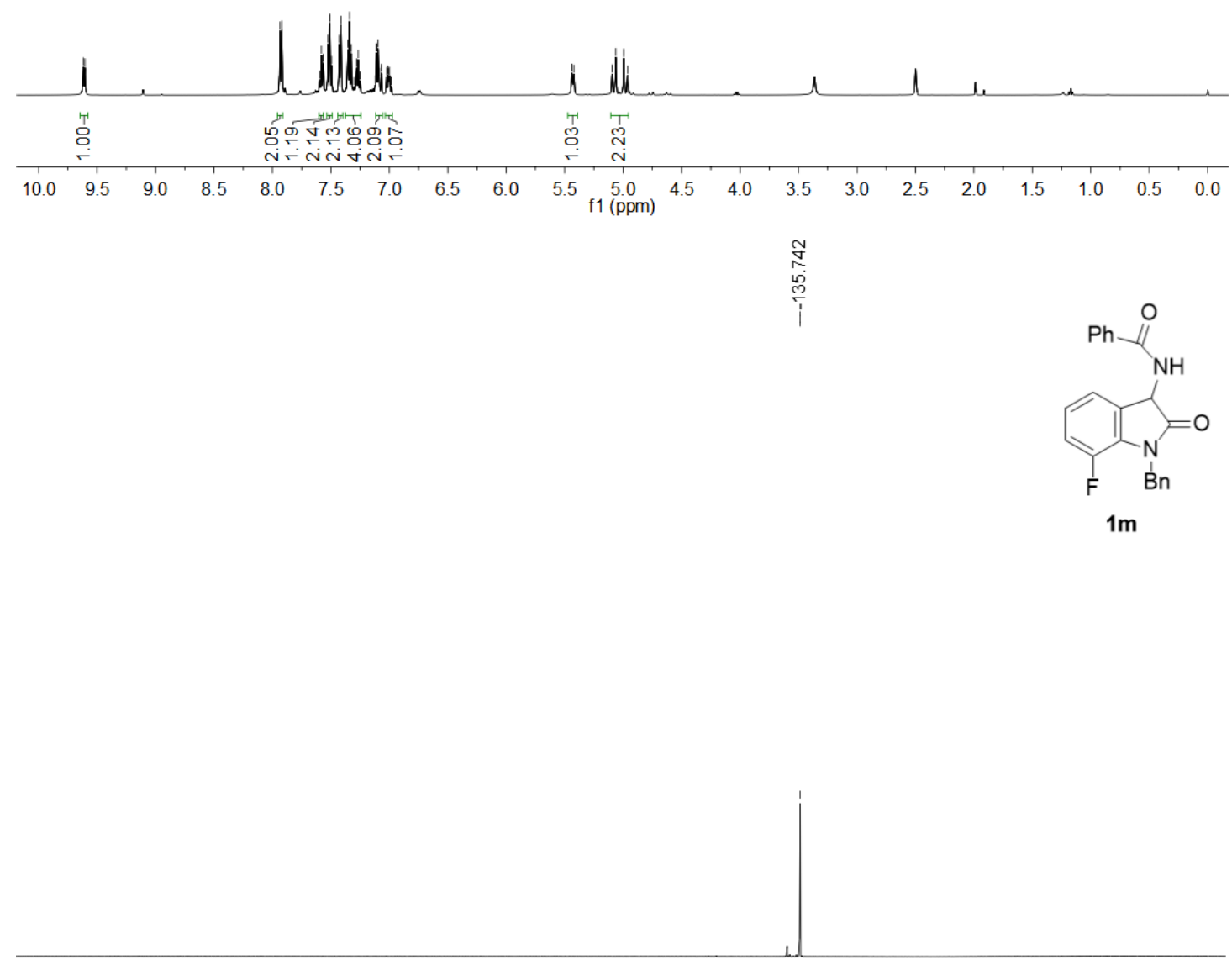

\begin{tabular}{lllllllllllllllllllll}
\hline 0 & 10 & 0 & -10 & -20 & -30 & -40 & -50 & -60 & -70 & -80 & -90 & & & -110 & -130 & -150 & -170 & -190 & -210
\end{tabular} 


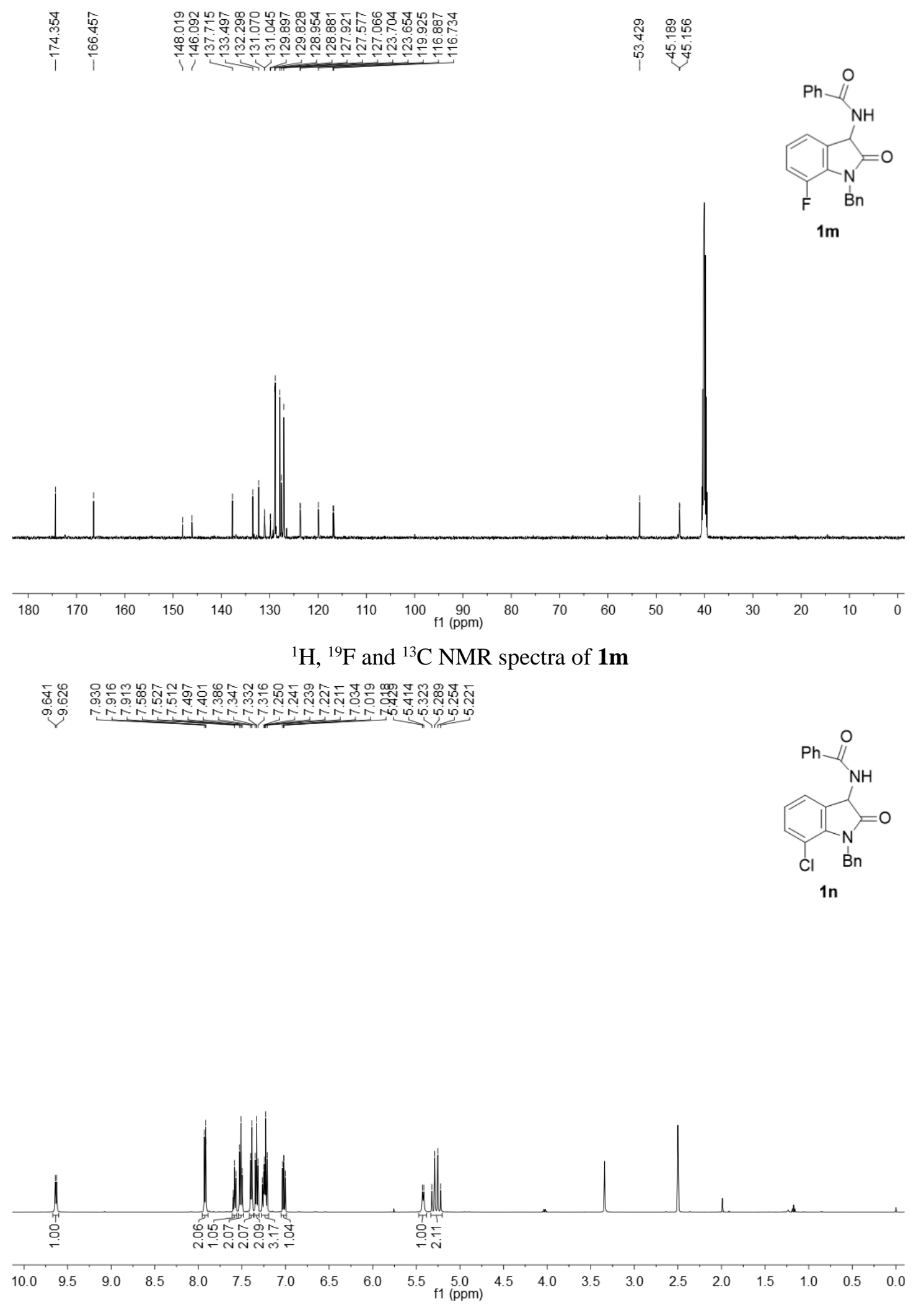




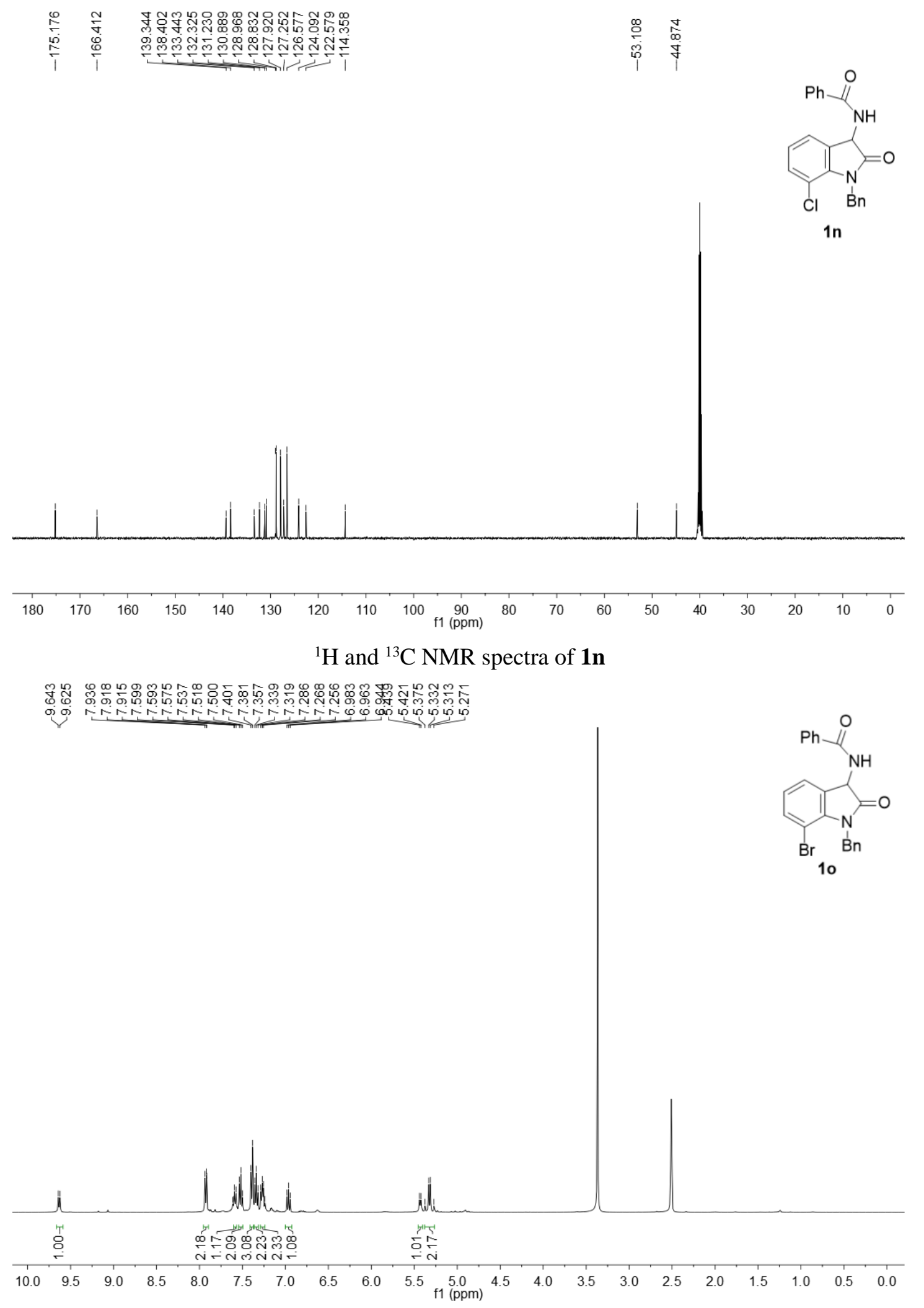




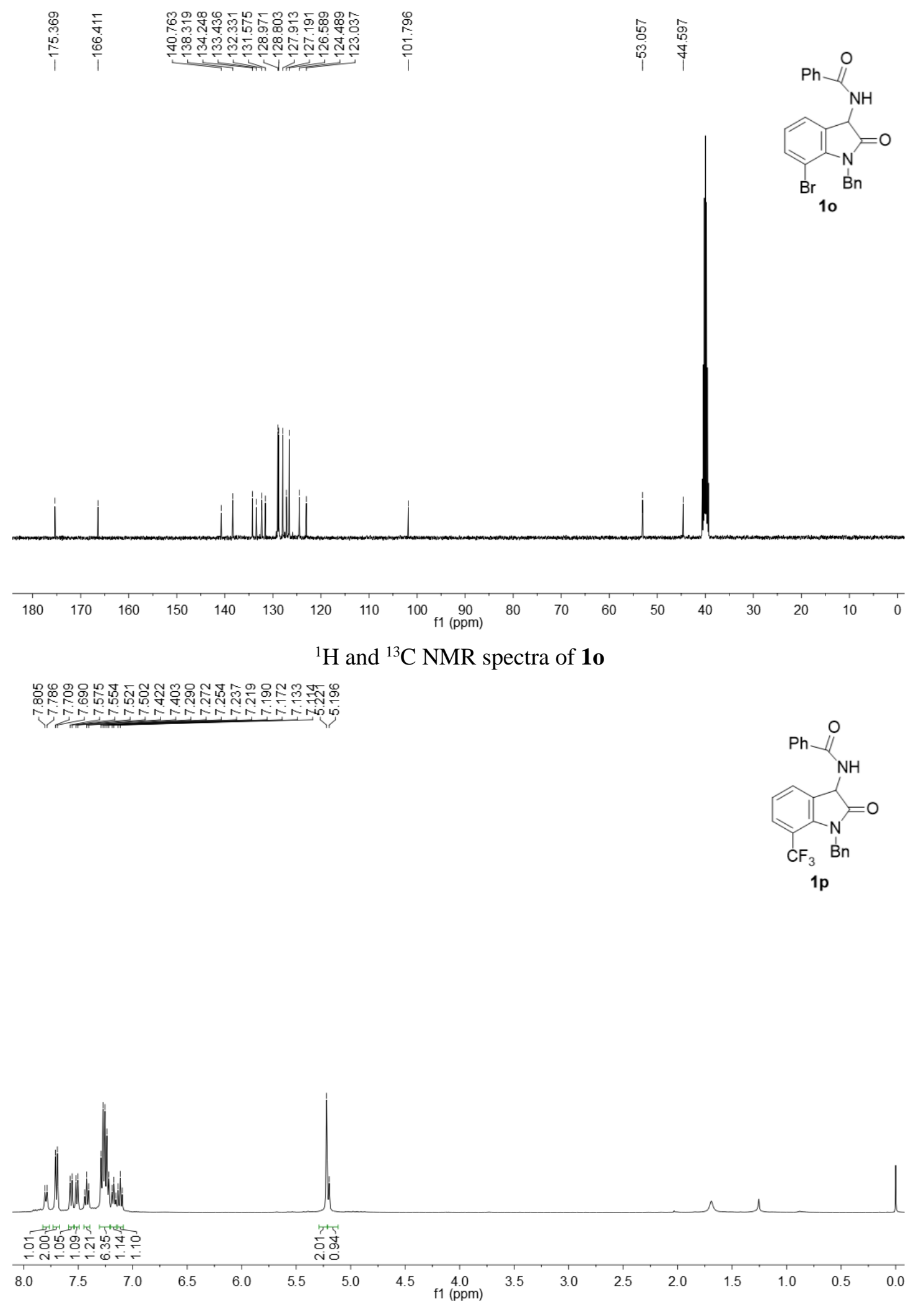



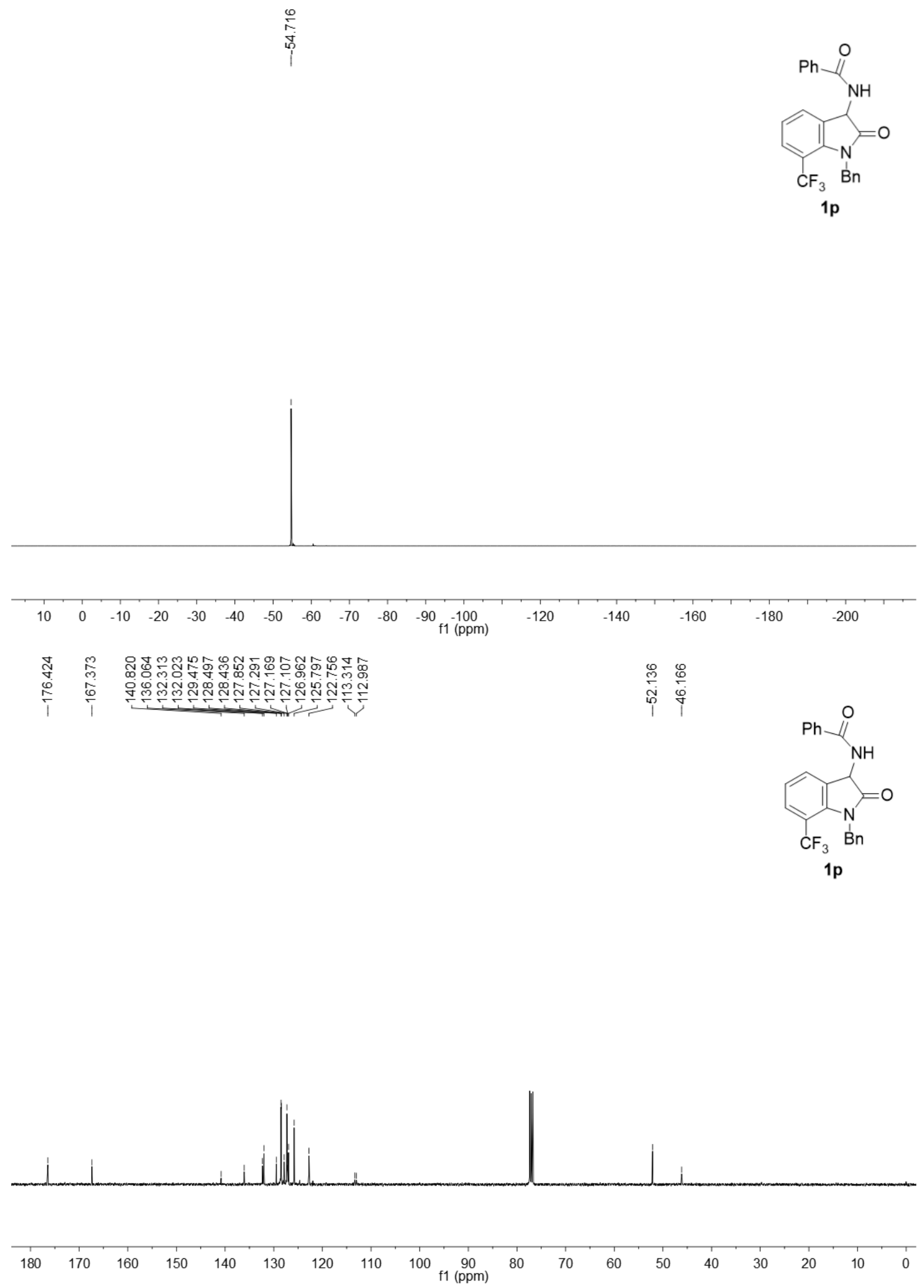

${ }^{1} \mathrm{H},{ }^{19} \mathrm{~F}$ and ${ }^{13} \mathrm{C}$ NMR spectra of $\mathbf{1 p}$ 


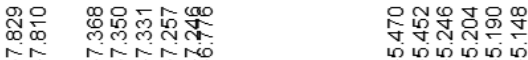

फी

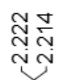
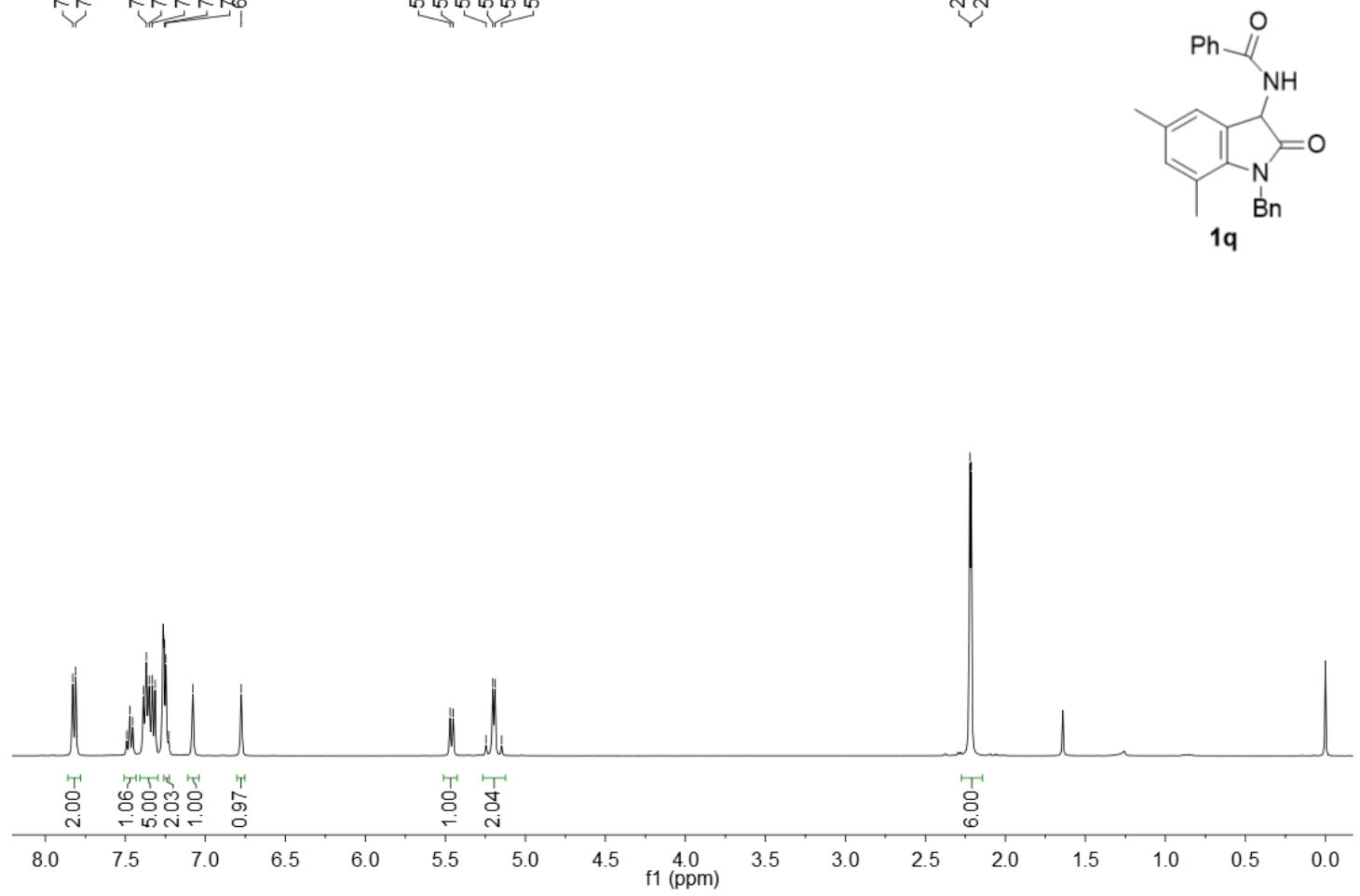

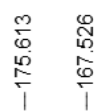

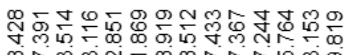

ס.

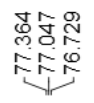

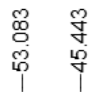

ஸิن
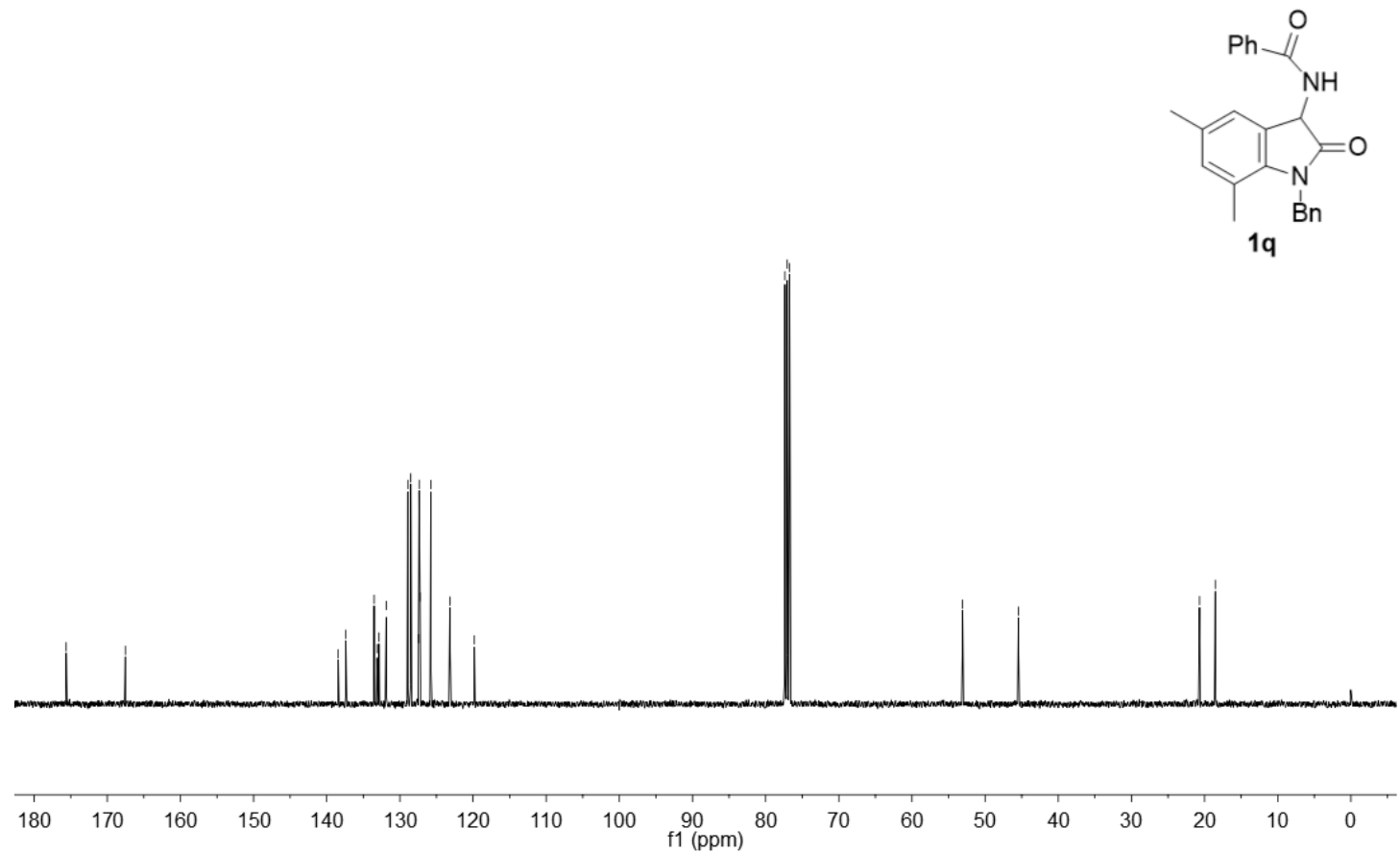

${ }^{1} \mathrm{H}$ and ${ }^{13} \mathrm{C}$ NMR spectra of $\mathbf{1 q}$ 


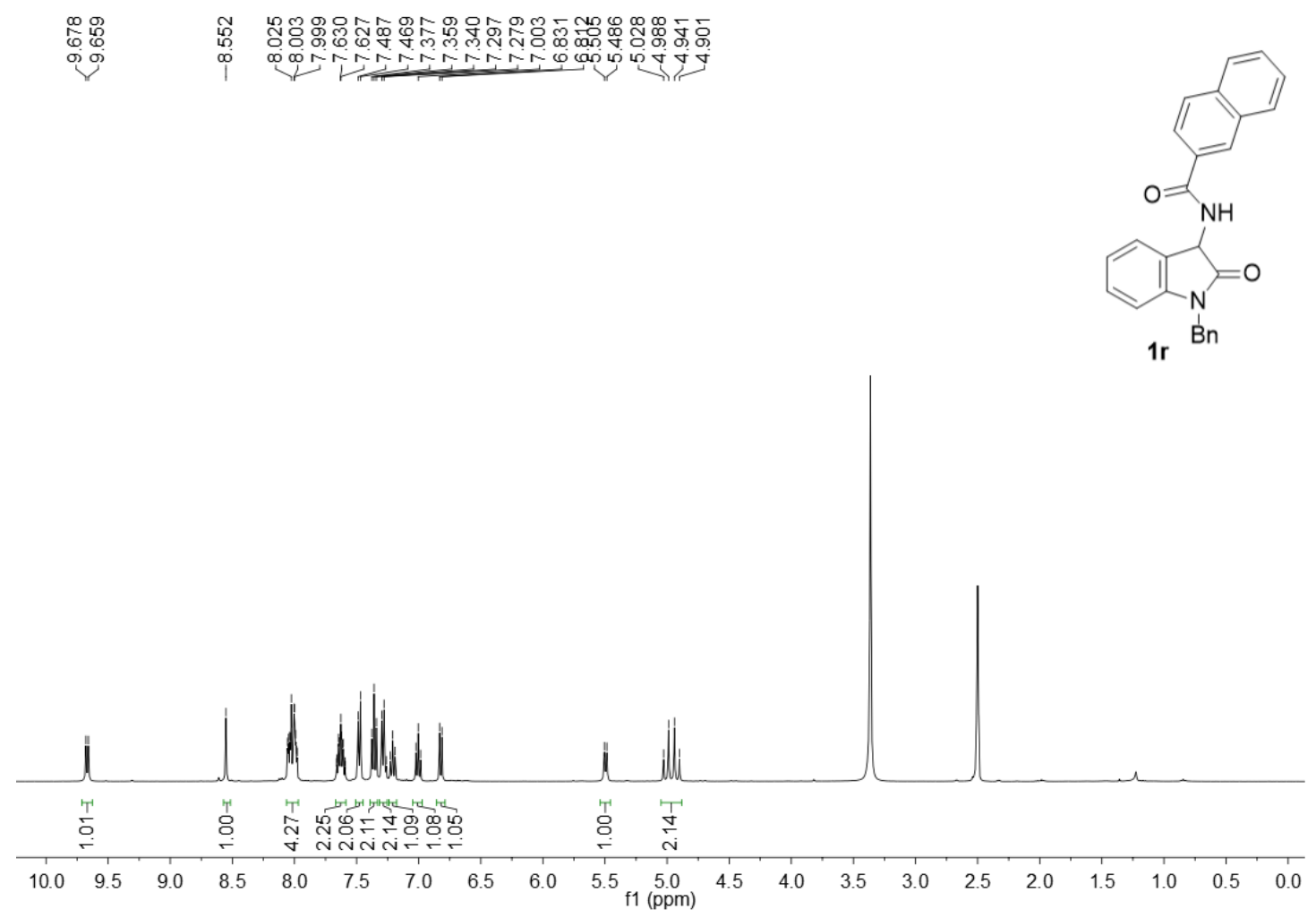

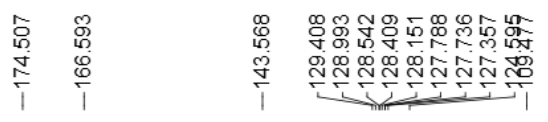

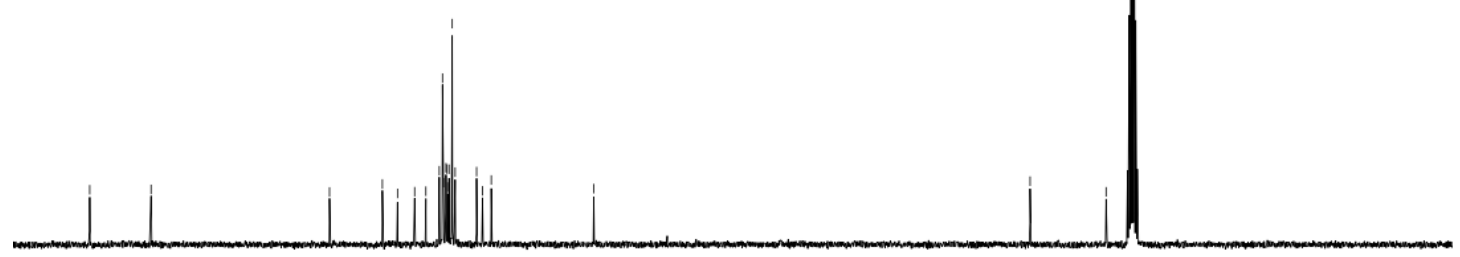

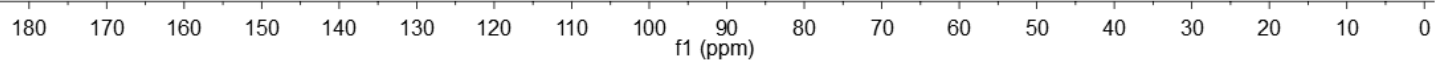

${ }^{1} \mathrm{H}$ and ${ }^{13} \mathrm{C}$ NMR spectra of $\mathbf{1 r}$ 

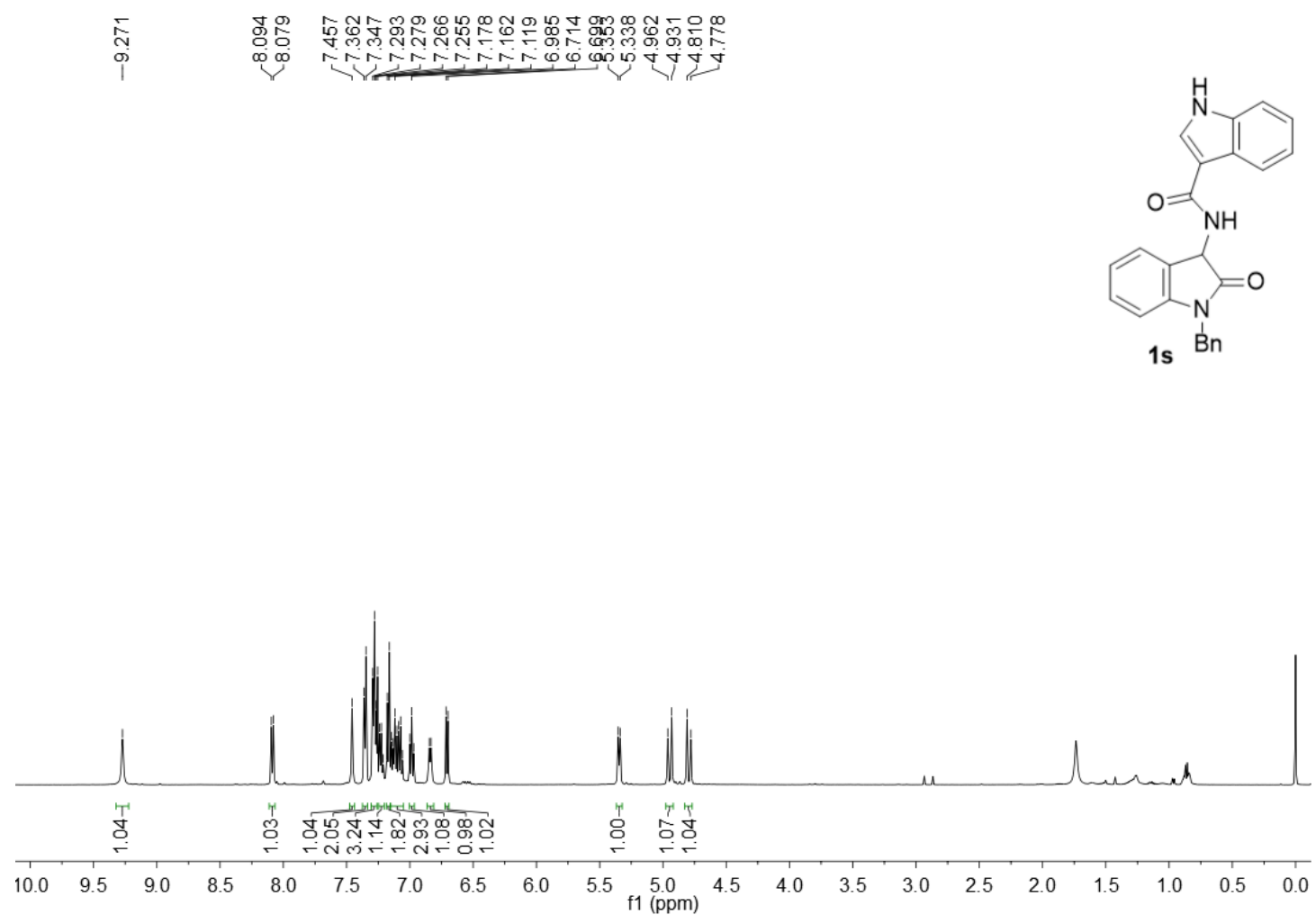

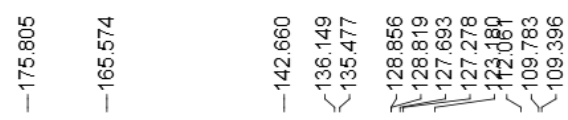

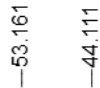
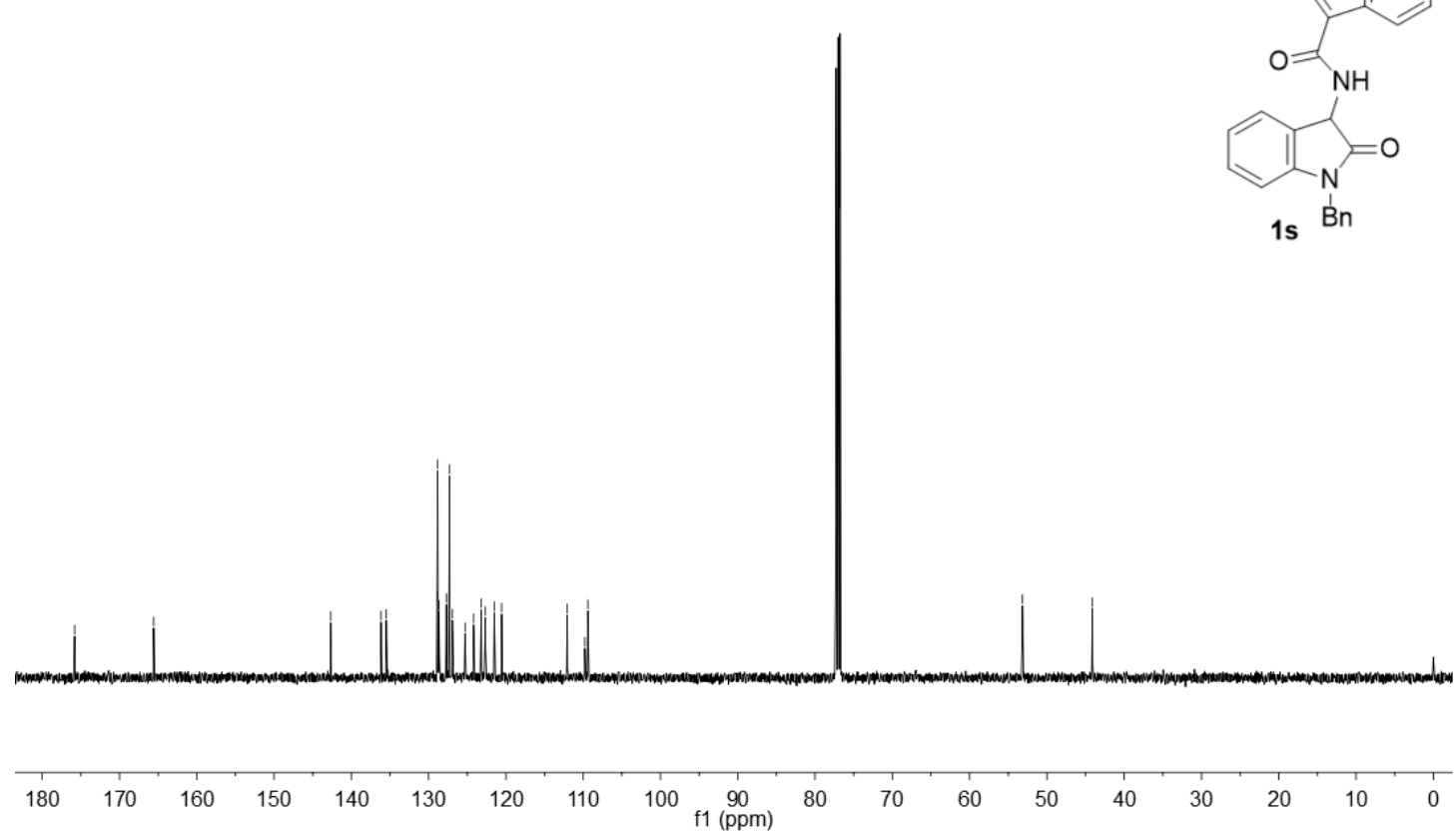

${ }^{1} \mathrm{H}$ and ${ }^{13} \mathrm{C}$ NMR spectra of $\mathbf{1 s}$ 

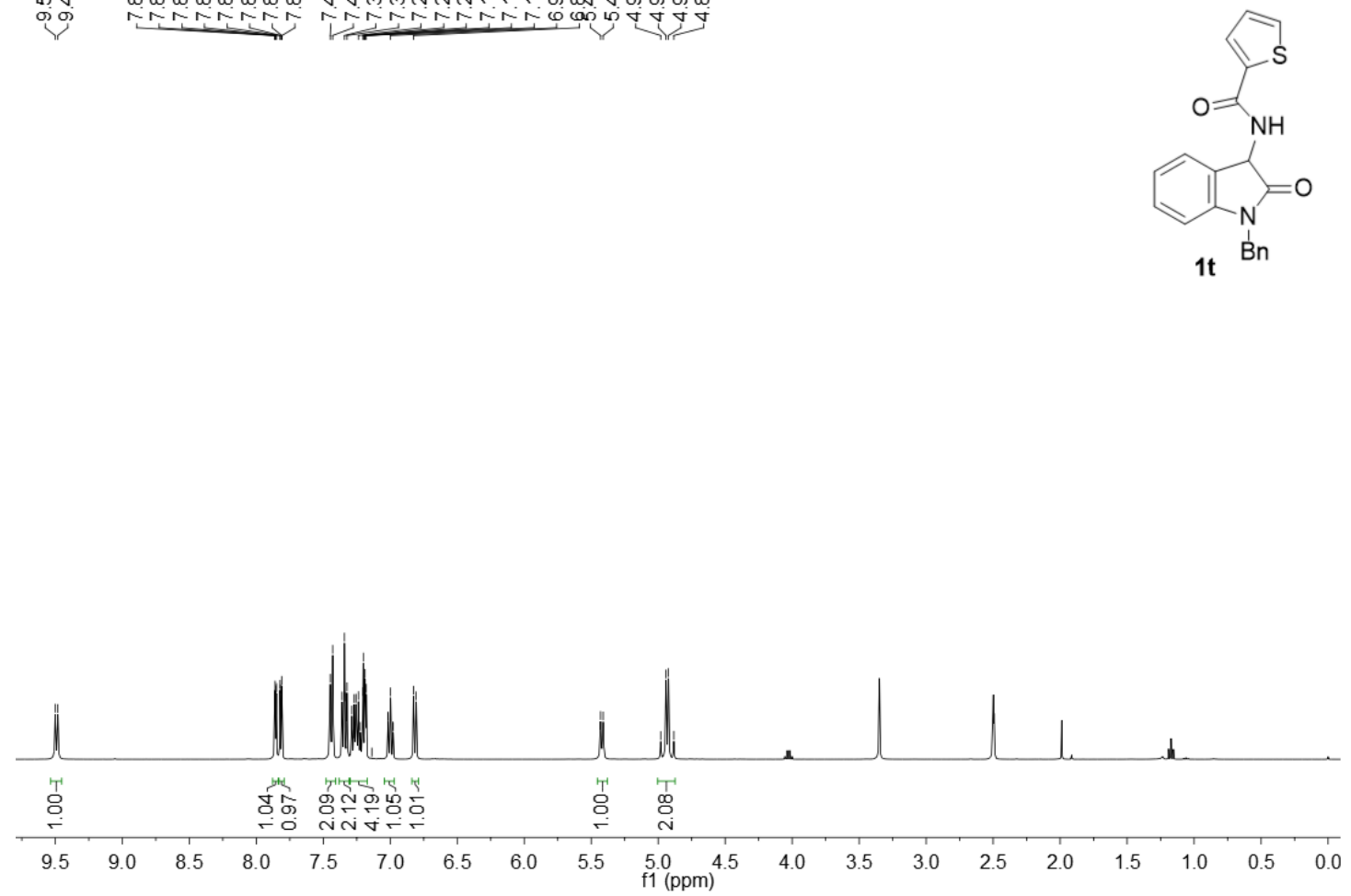

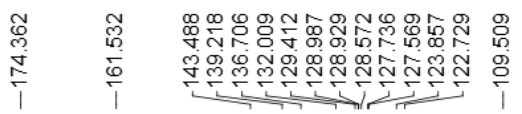
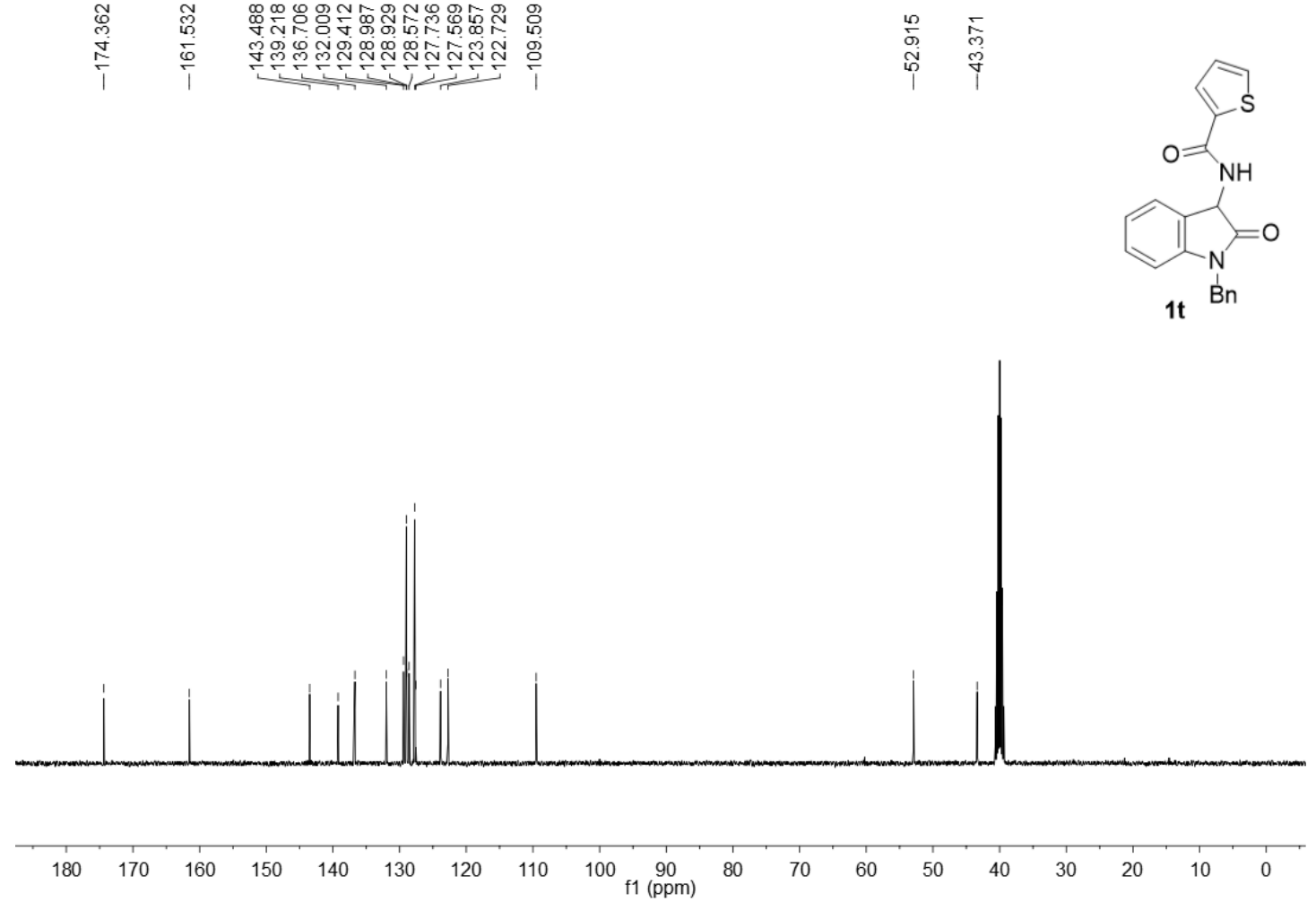

${ }^{1} \mathrm{H}$ and ${ }^{13} \mathrm{C}$ NMR spectra of $\mathbf{1 t}$ 


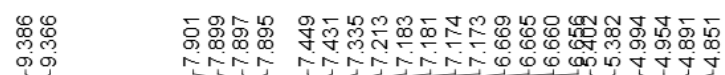

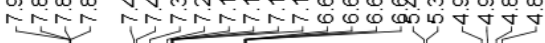

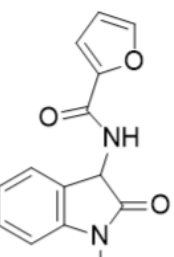

$1 \mathrm{u}^{\mathrm{Bn}}$

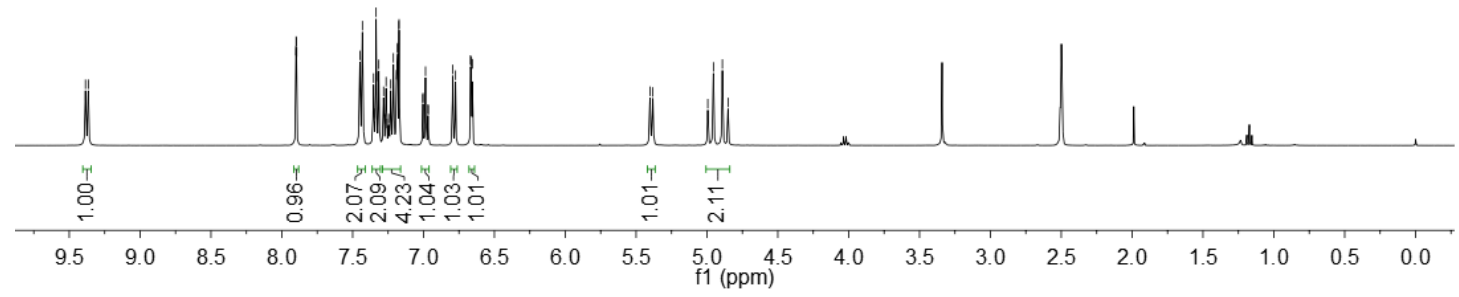

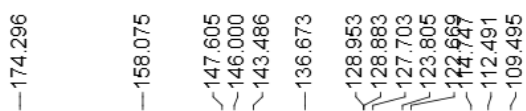

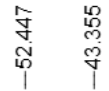

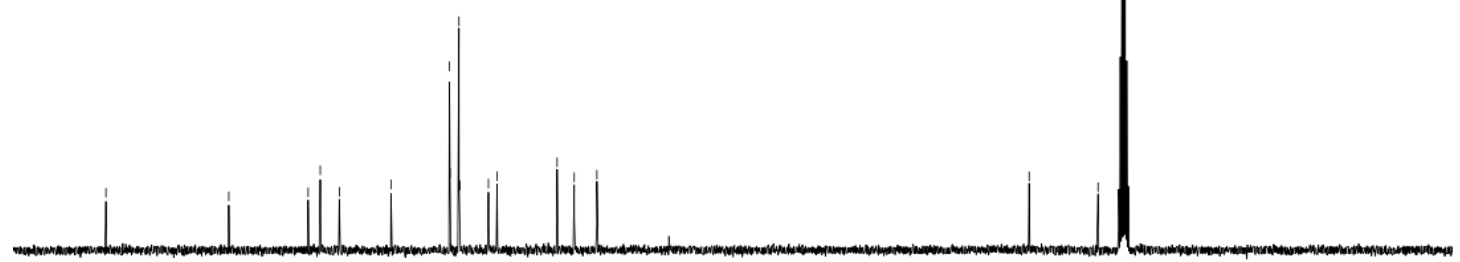

$\begin{array}{llllllllllllllllll}180 & 170 & 160 & 150 & 140 & 130 & 120 & 110 & 100 \begin{array}{l}90 \\ \mathrm{f} 1(\mathrm{ppm})\end{array} & 80 & 70 & 60 & 50 & 40 & 30 & 20 & 10 & 0\end{array}$

${ }^{1} \mathrm{H}$ and ${ }^{13} \mathrm{C}$ NMR spectra of $\mathbf{1 u}$ 

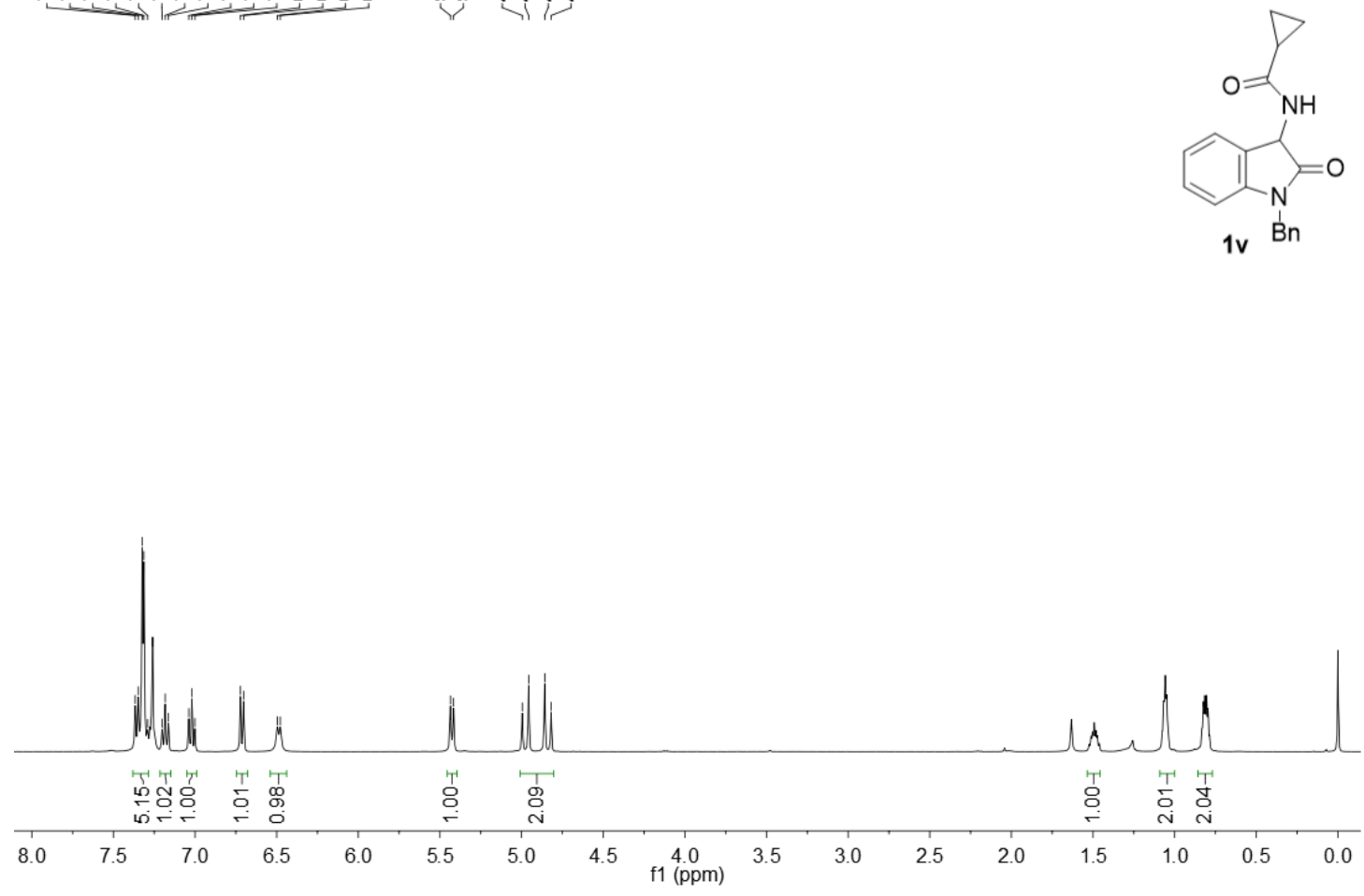

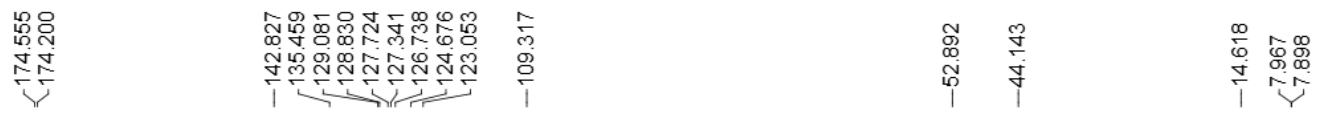

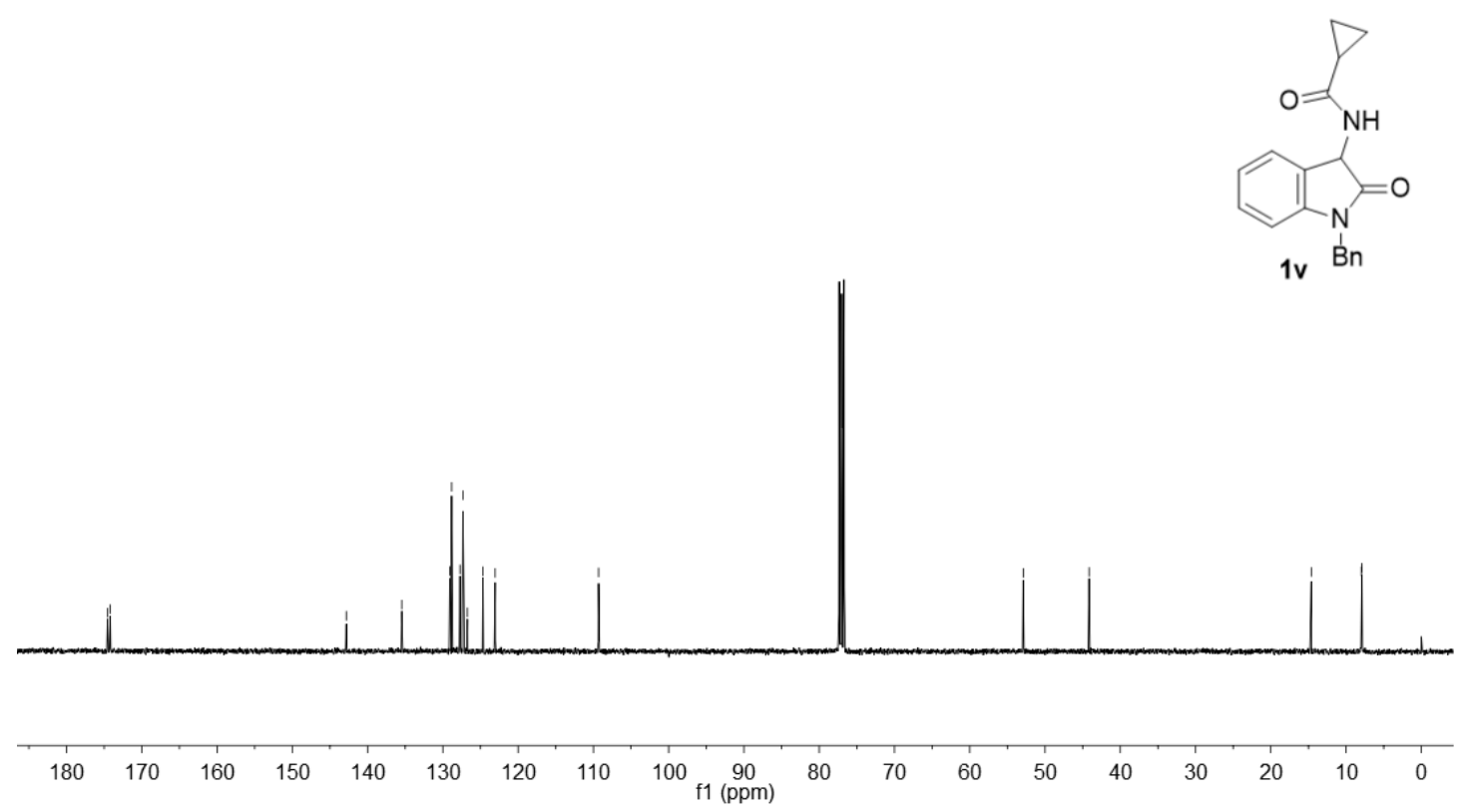

${ }^{1} \mathrm{H}$ and ${ }^{13} \mathrm{C}$ NMR spectra of $\mathbf{1 v}$ 


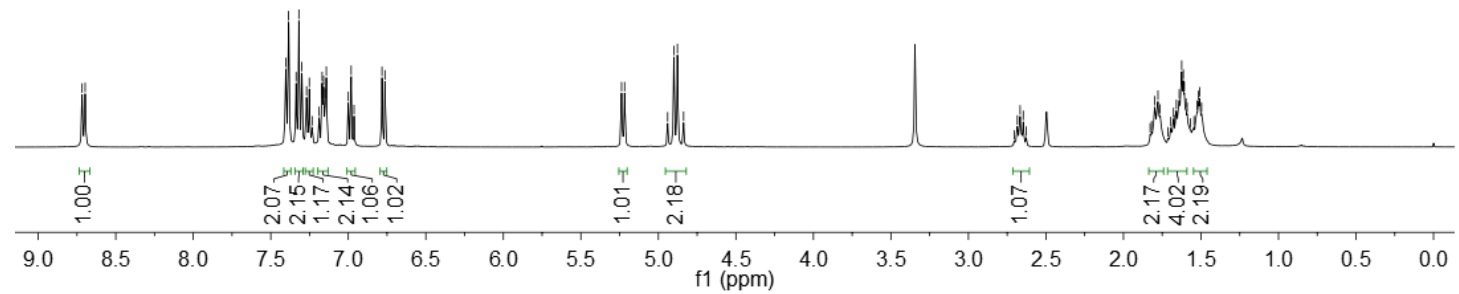

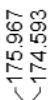

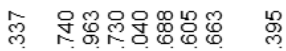
।

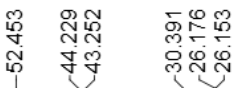
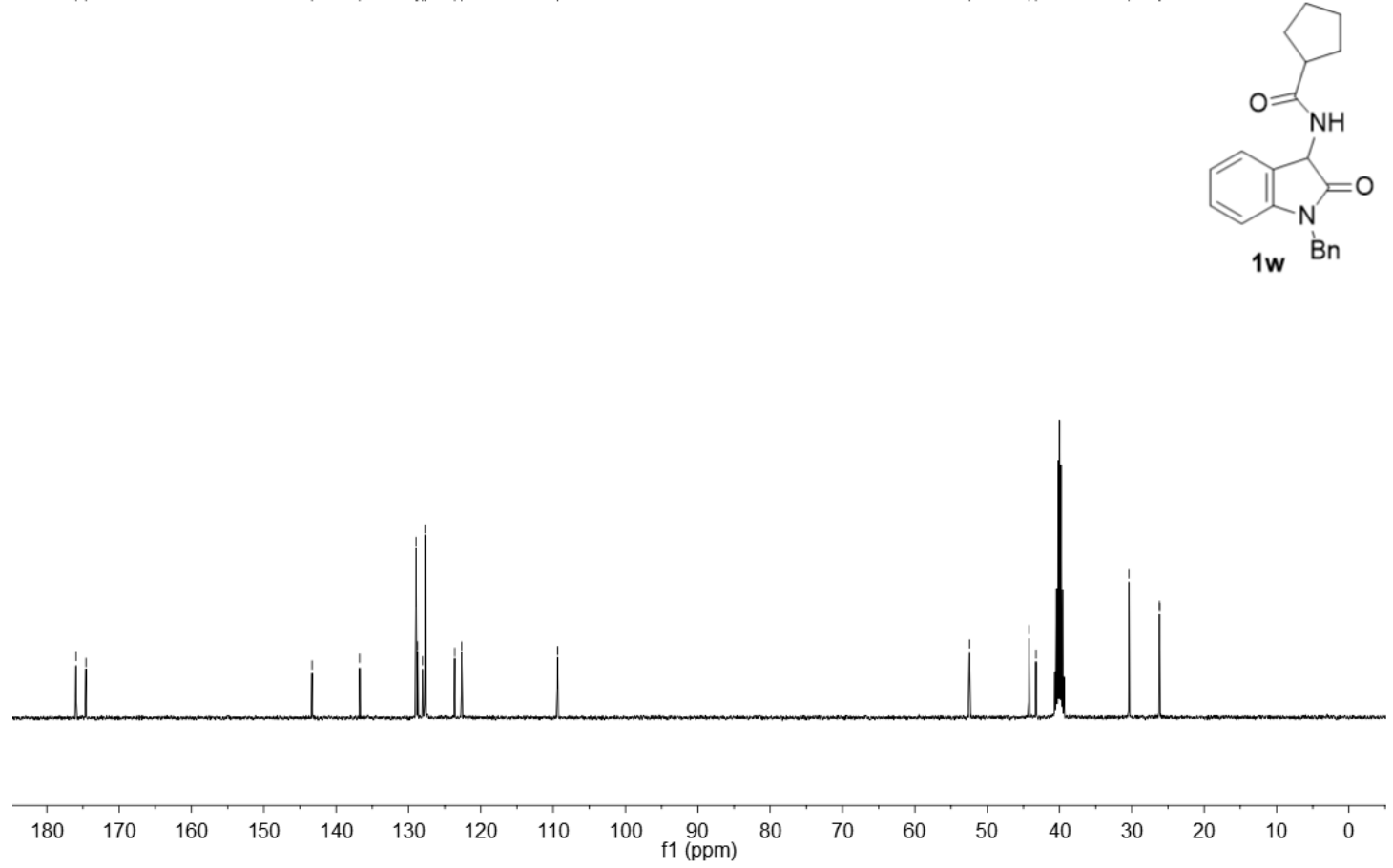

${ }^{1} \mathrm{H}$ and ${ }^{13} \mathrm{C}$ NMR spectra of $\mathbf{1 w}$ 

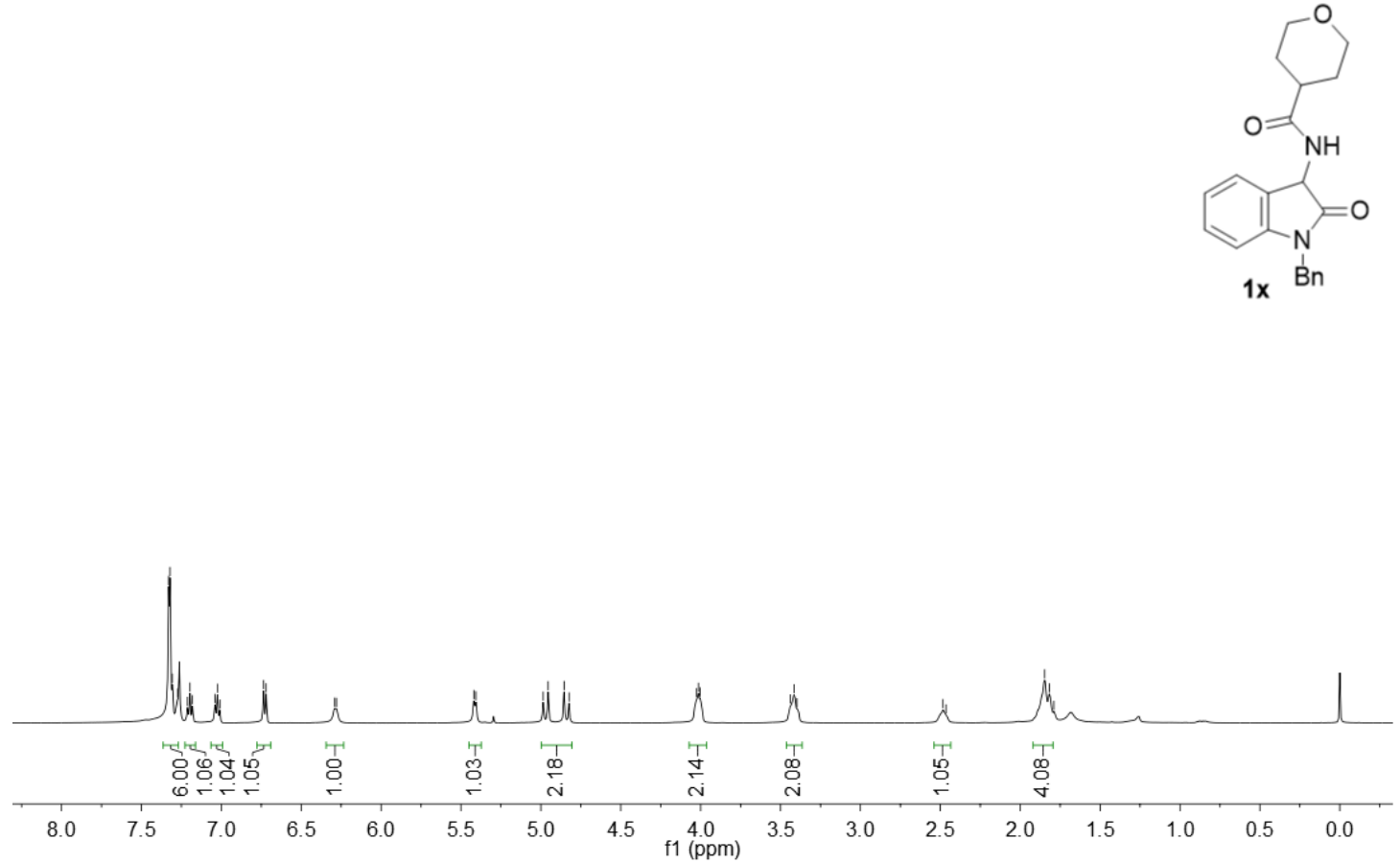

要
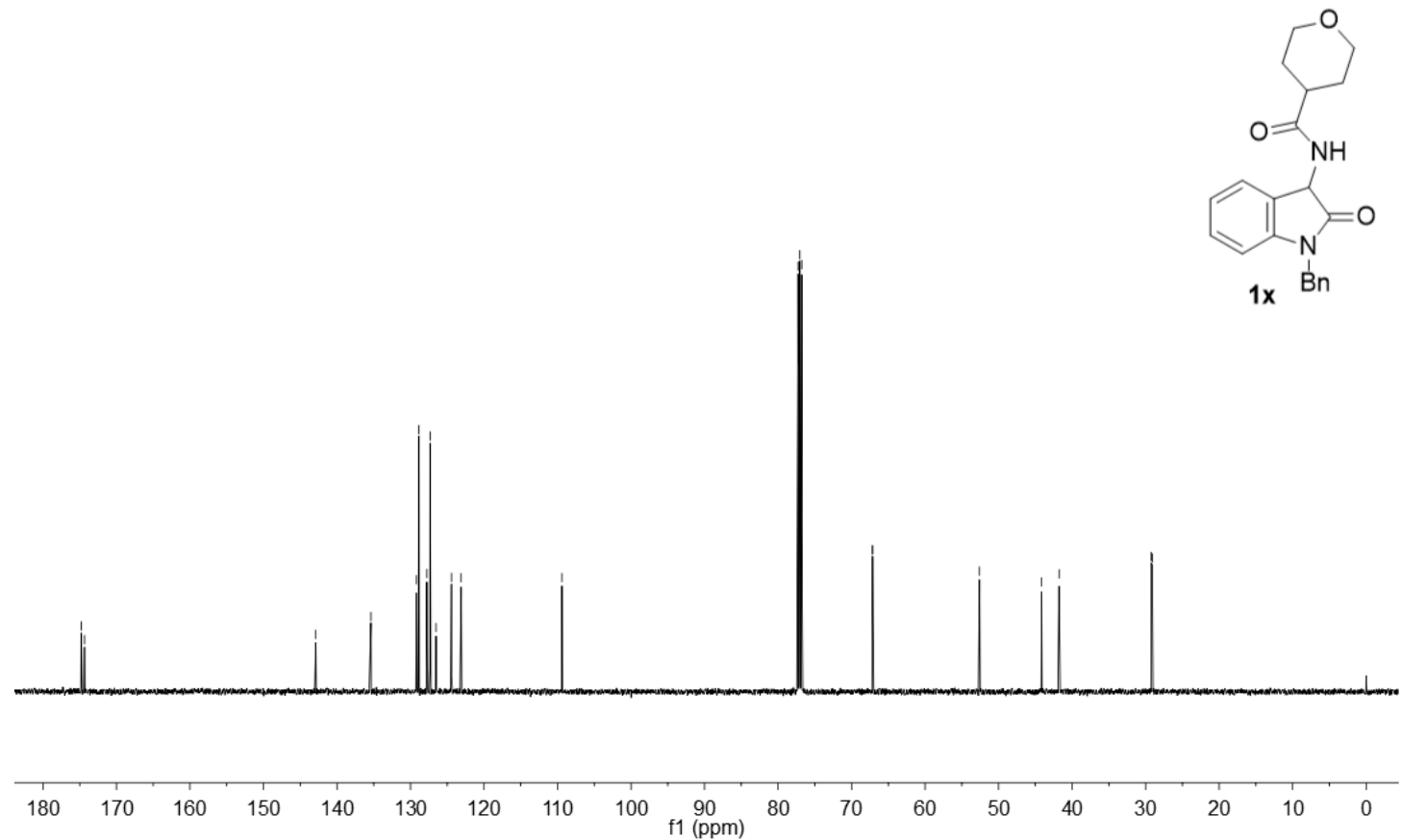

${ }^{1} \mathrm{H}$ and ${ }^{13} \mathrm{C}$ NMR spectra of $\mathbf{1} \mathbf{x}$ 

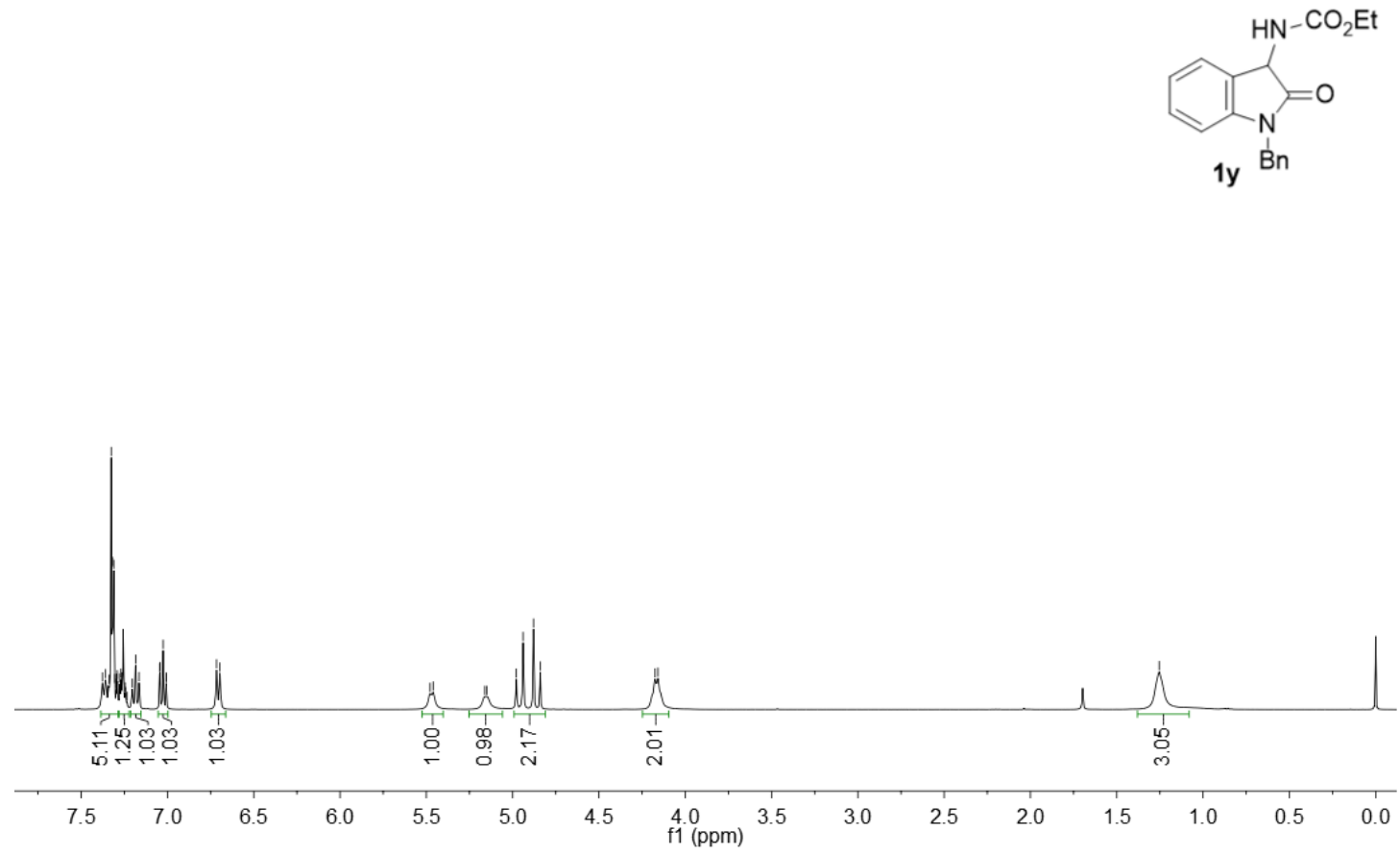

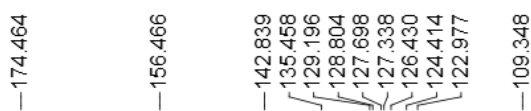

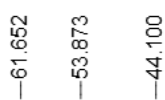

$\underset{\substack{i \\ \stackrel{\infty}{j}}}{\stackrel{i}{j}}$

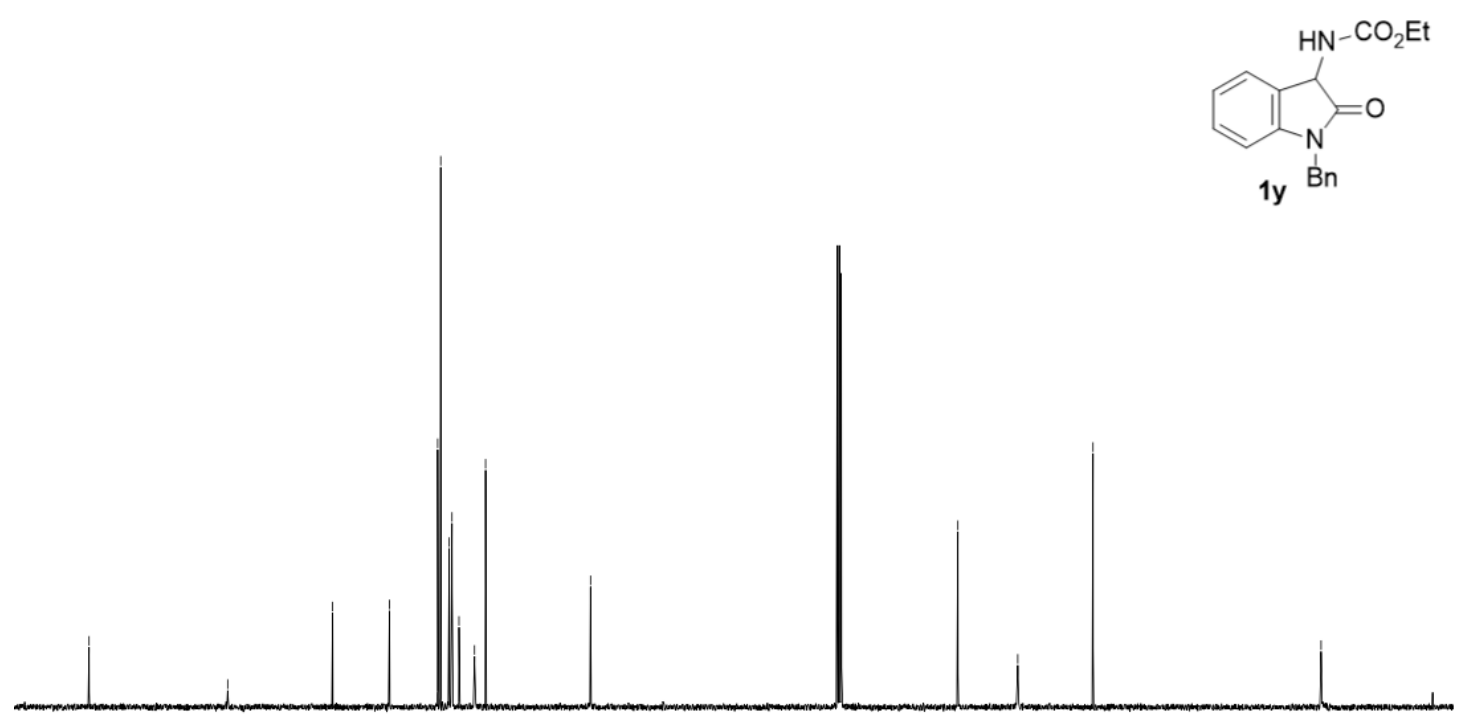

$\begin{array}{lllllllllllllllllll}180 & 170 & 160 & 150 & 140 & 130 & 120 & 110 & 100 & \begin{array}{c}90 \\ \mathrm{f} 1(\mathrm{ppm})\end{array} & 80 & 70 & 60 & 50 & 40 & 30 & 20 & 10 & 0\end{array}$

${ }^{1} \mathrm{H}$ and ${ }^{13} \mathrm{C}$ NMR spectra of $\mathbf{1 y}$ 


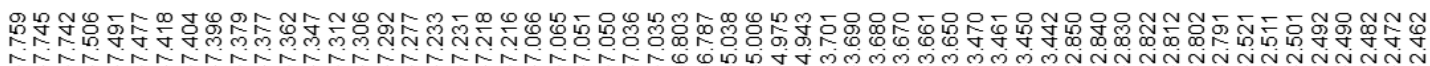

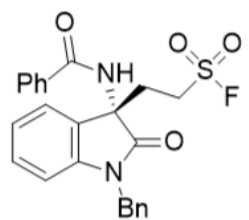

$3 a$

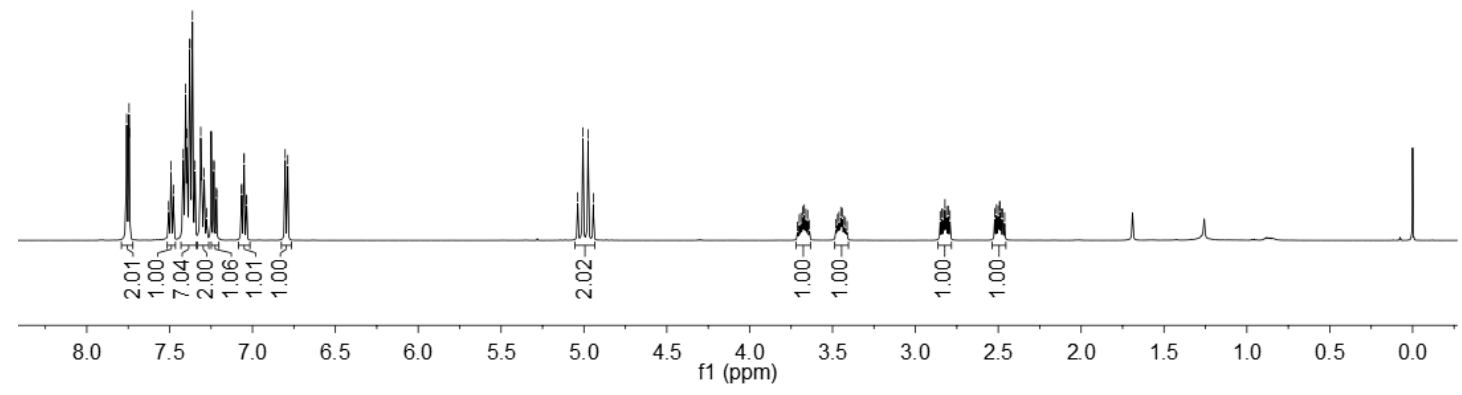

ֻ

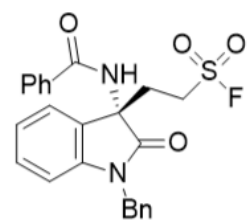

$3 a$

$\begin{array}{llllllllllllllllllllll}20 & 110 & 100 & 90 & 80 & 70 & 60 & 50 & 40 & 30 & 20 & 10 & 0 \\ \mathrm{f} 1(\mathrm{ppm}) & -10 & -20 & -30 & -40 & -50 & -60 & -70 & -80 & -90 & -110\end{array}$ 


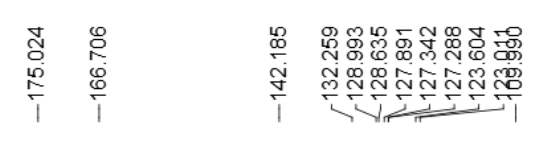

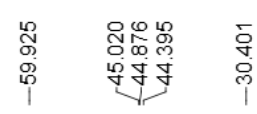

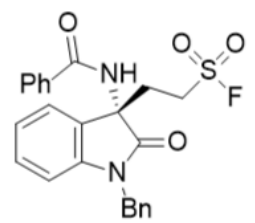

3 a

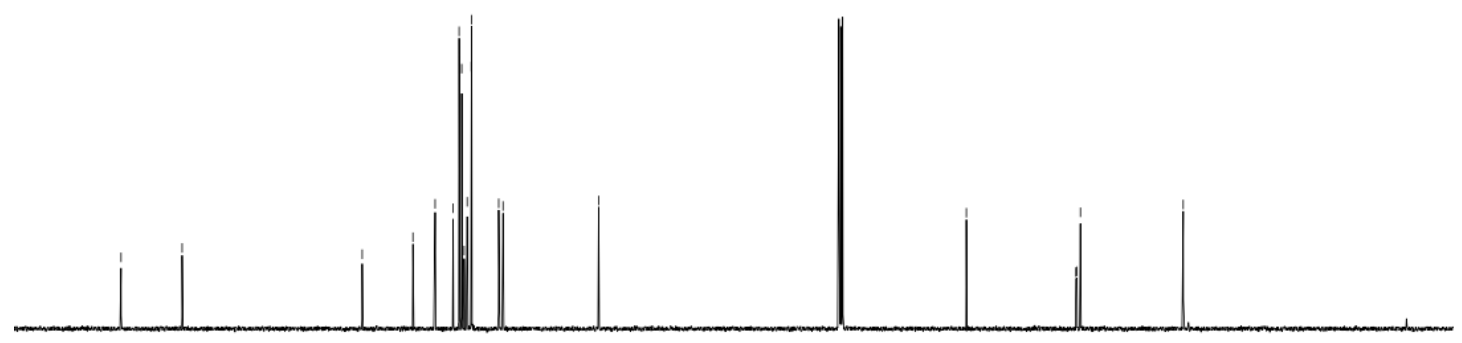

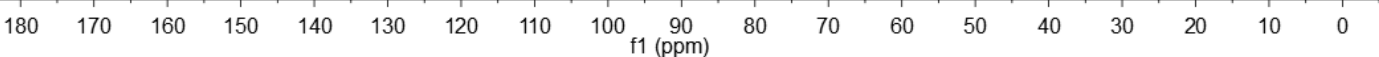

${ }^{1} \mathrm{H},{ }^{19} \mathrm{~F}$ and ${ }^{13} \mathrm{C}$ NMR spectra of 3a

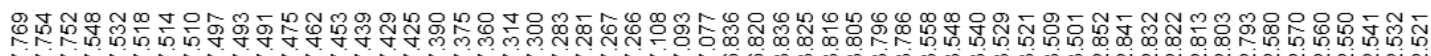

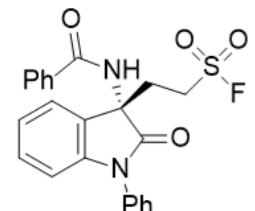

$3 b$

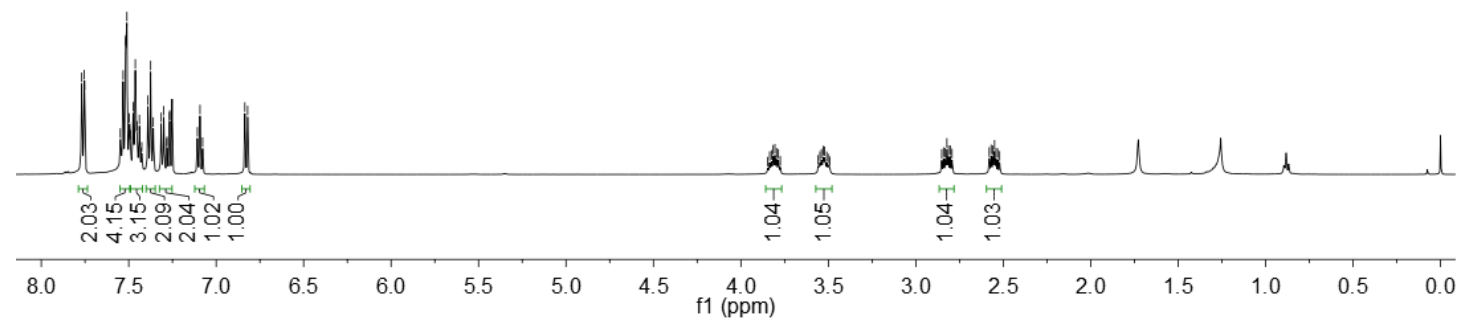




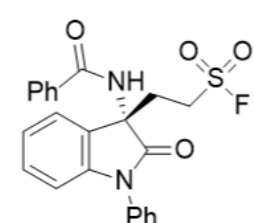

$3 b$

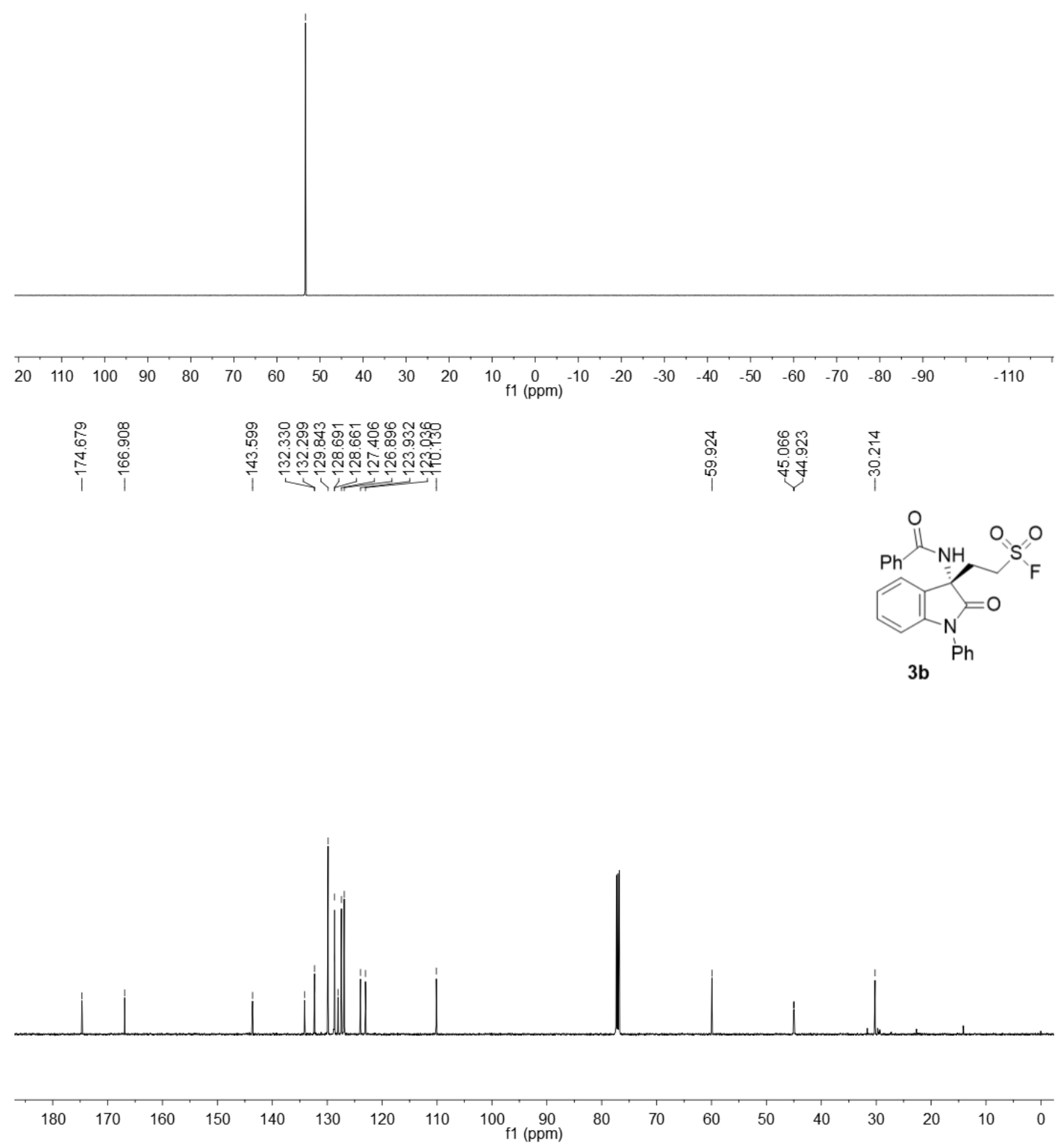

${ }^{1} \mathrm{H},{ }^{19} \mathrm{~F}$ and ${ }^{13} \mathrm{C}$ NMR spectra of $\mathbf{3 b}$ 


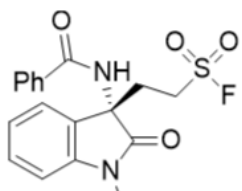

$3 c$

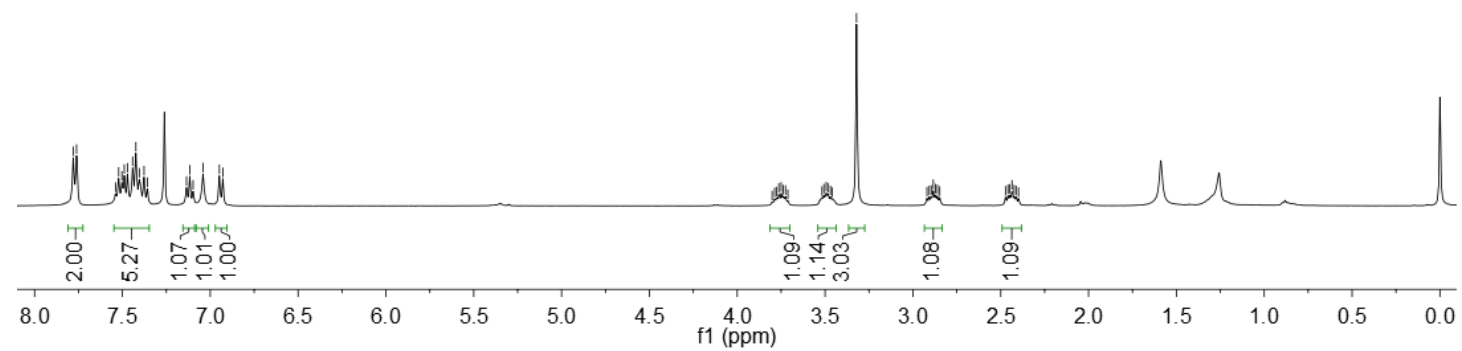

$\frac{\infty}{\infty}$
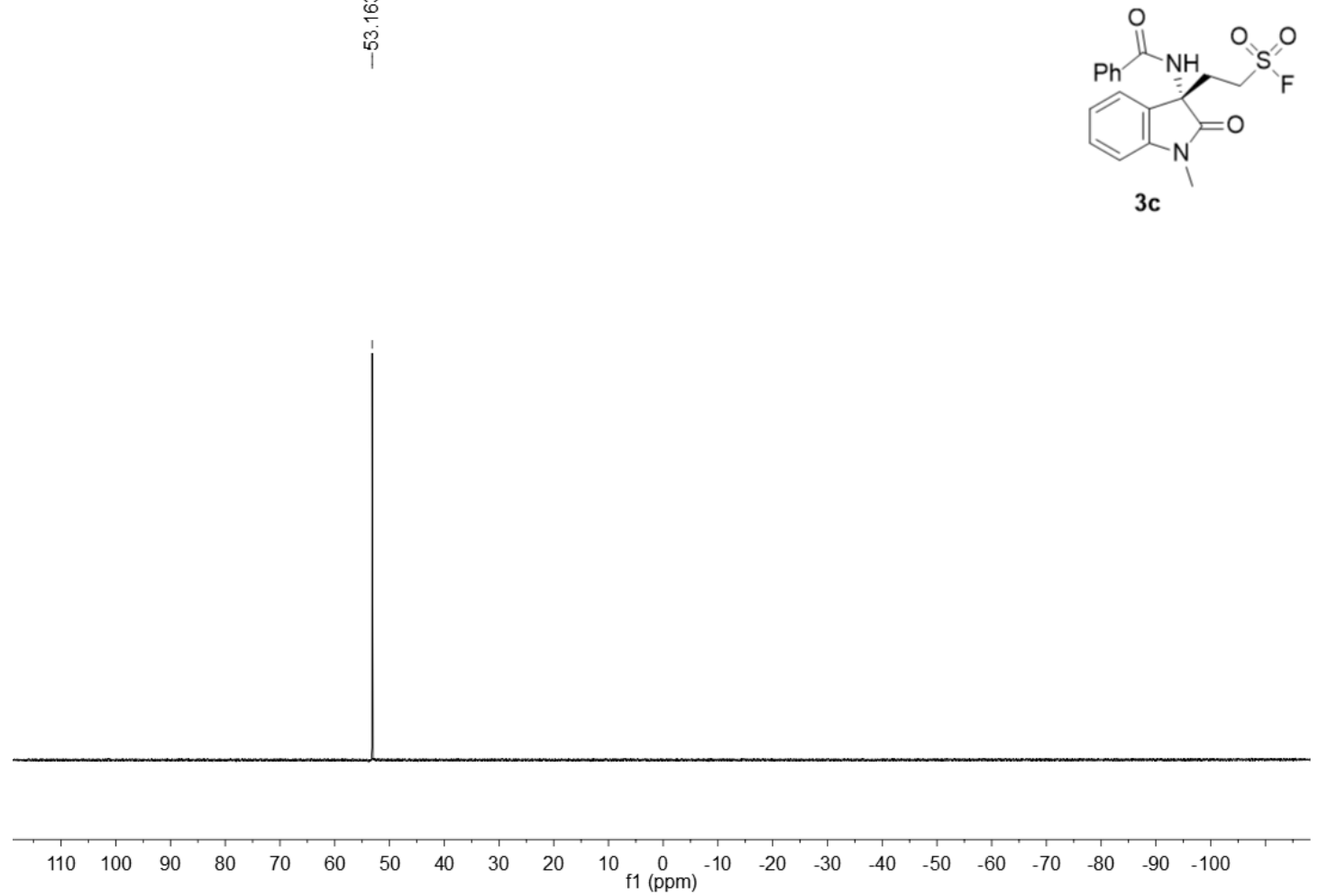


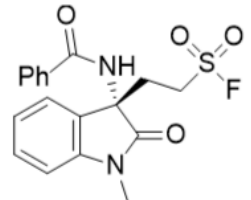

$3 c$
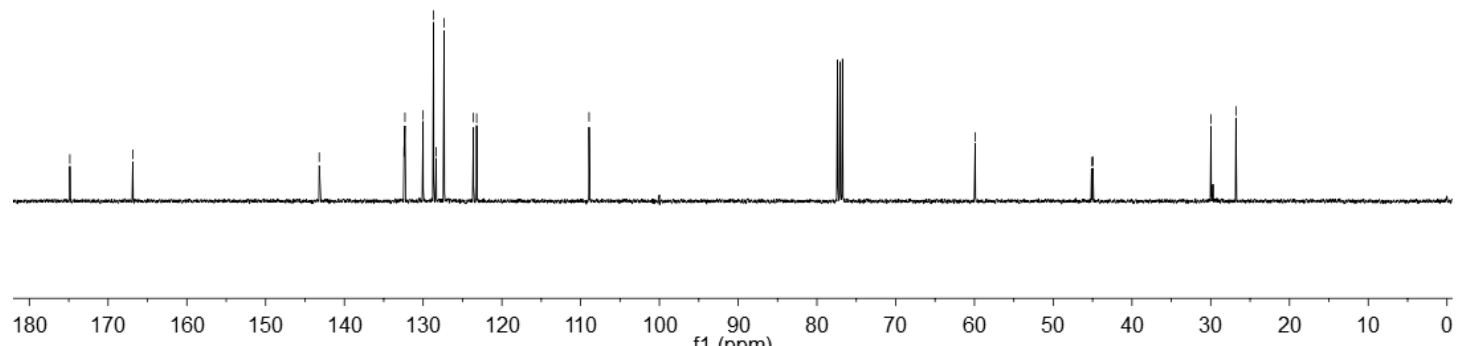

${ }^{1} \mathrm{H},{ }^{19} \mathrm{~F}$ and ${ }^{13} \mathrm{C}$ NMR spectra of $3 \mathrm{c}$

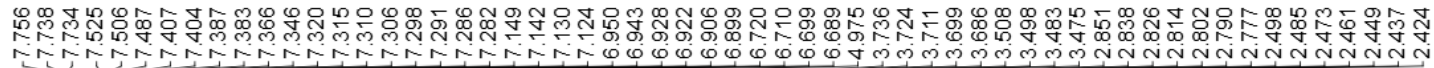

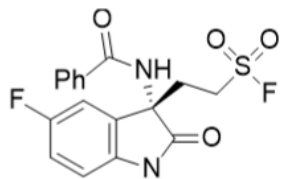

$3 d$

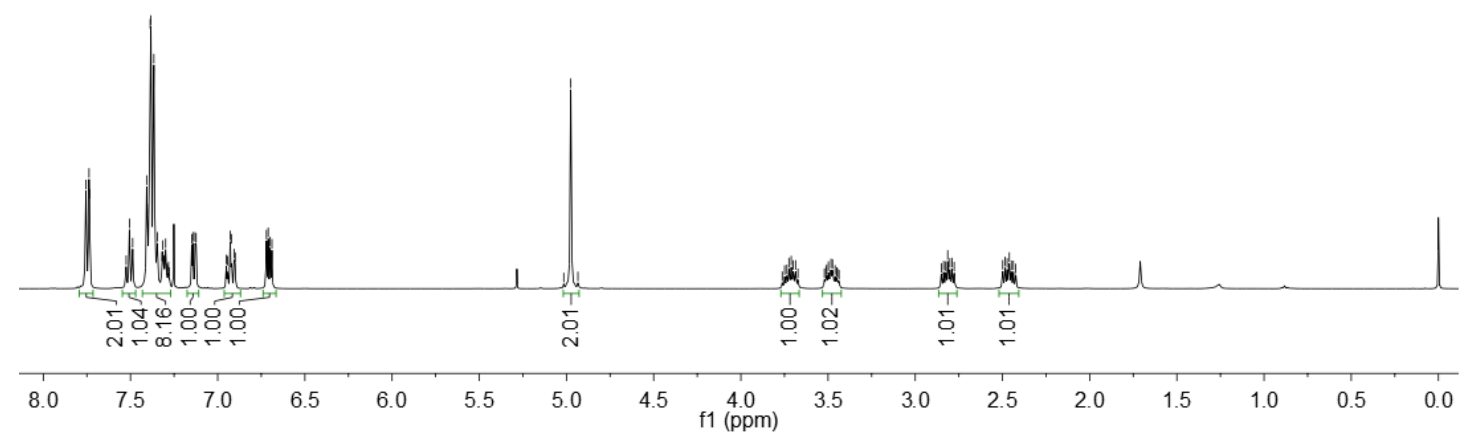


্ֻল

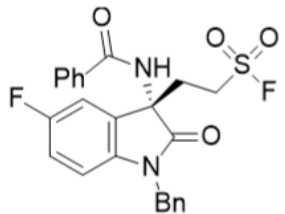

3d

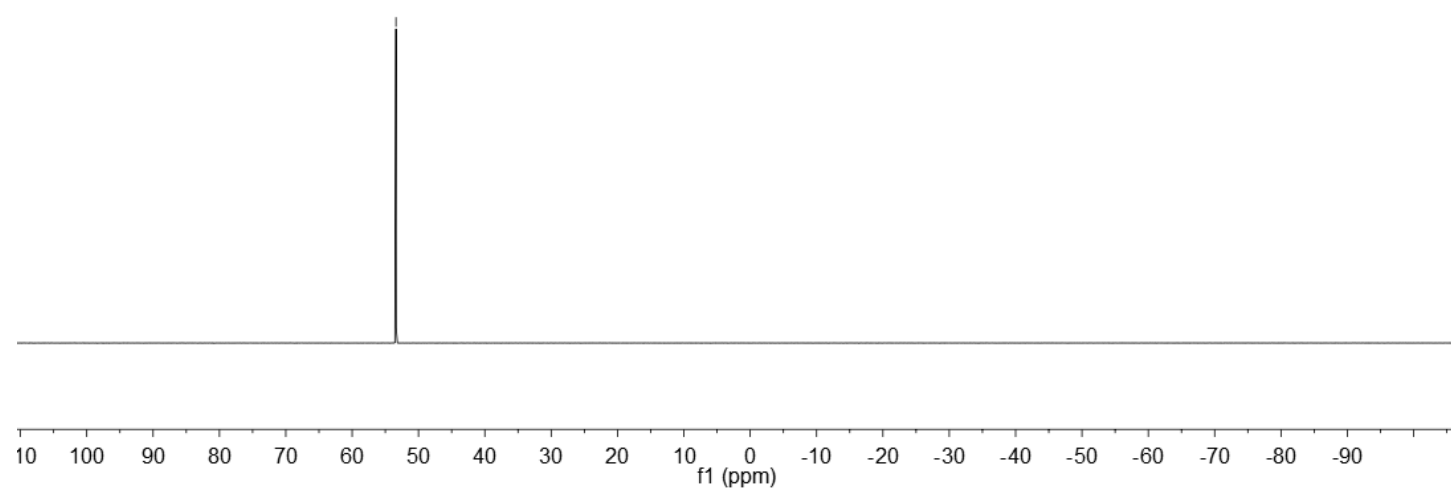

峁

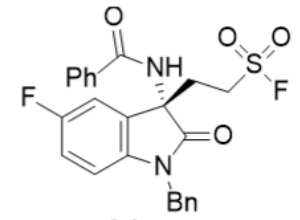

3d

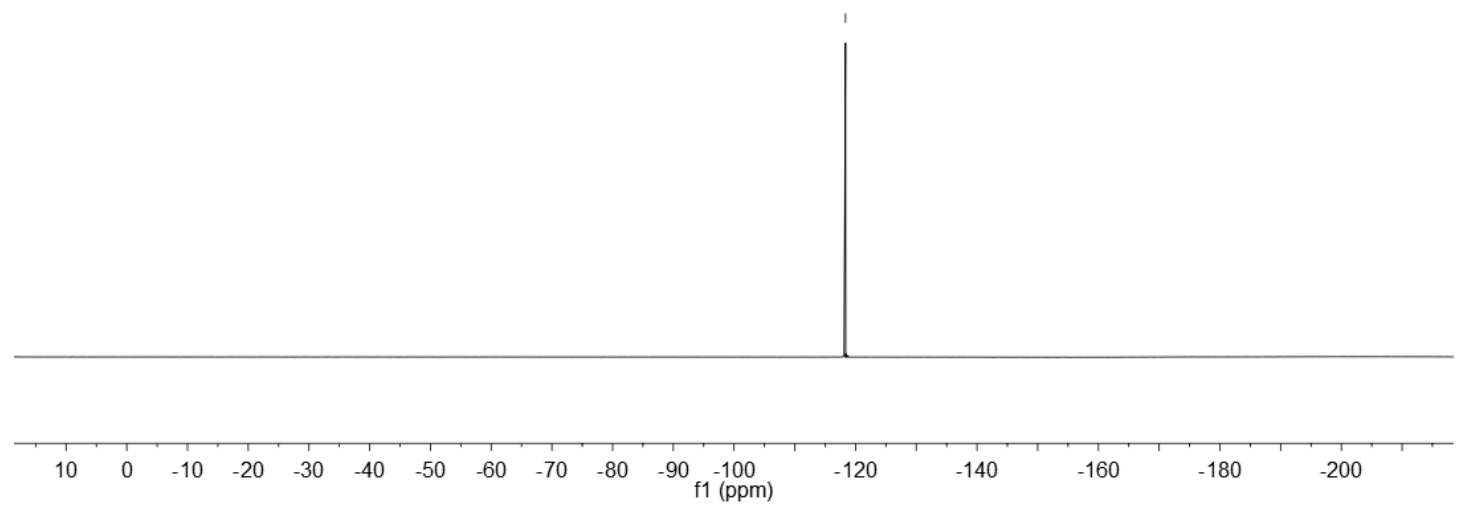




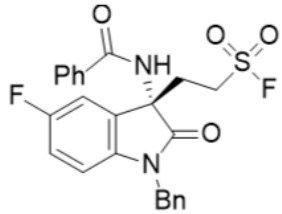

$3 d$

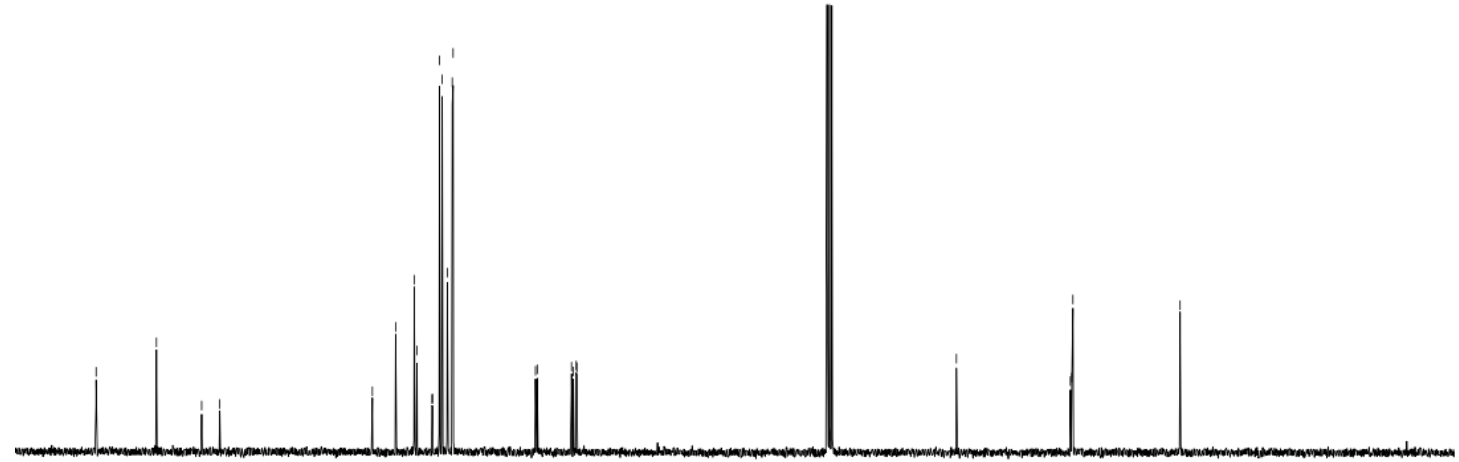

$\begin{array}{lllllllllllllllllll}180 & 170 & 160 & 150 & 140 & 130 & 120 & 110 & 100 & 90 & 80 & 70 & 60 & 50 & 40 & 30 & 20 & 10 & 0\end{array}$

${ }^{1} \mathrm{H},{ }^{19} \mathrm{~F}$ and ${ }^{13} \mathrm{C}$ NMR spectra of $\mathbf{3 d}$

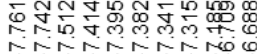

0
0
0

rininano

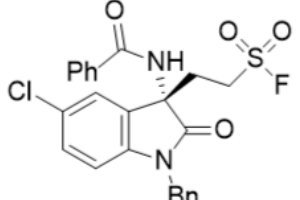

$3 e$

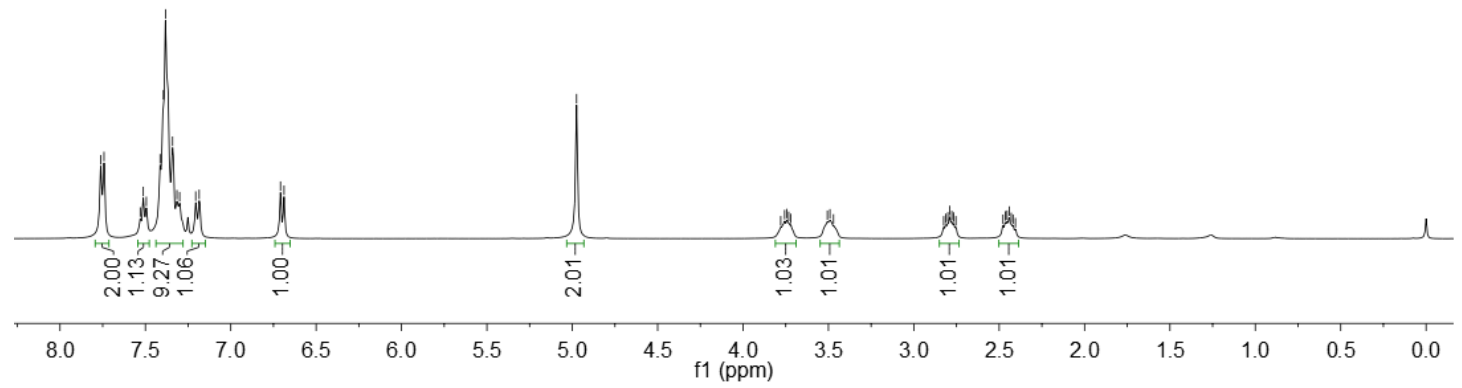



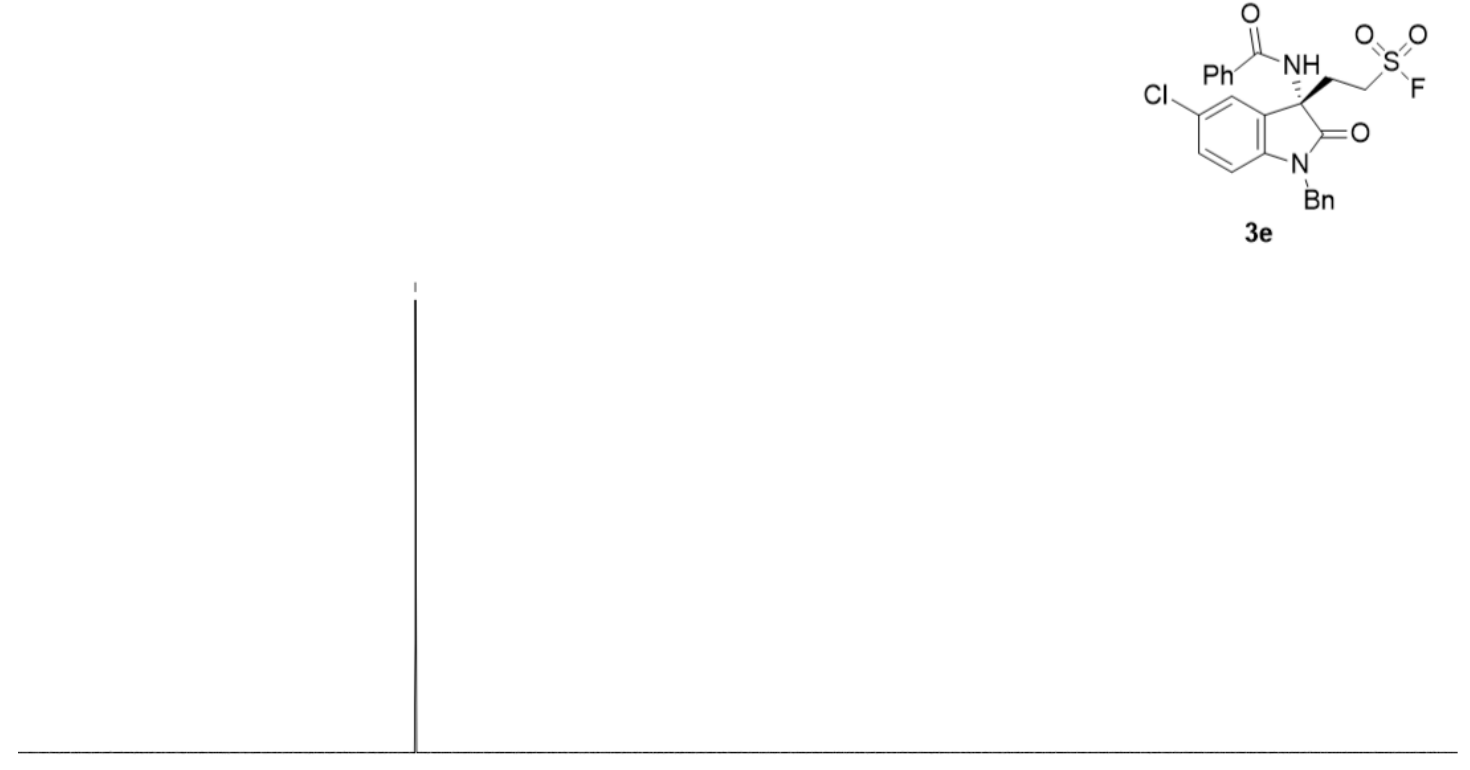

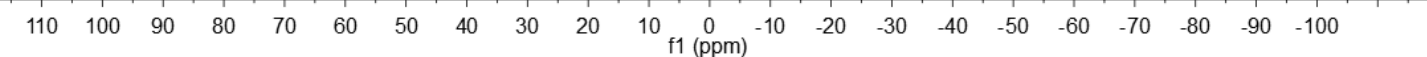

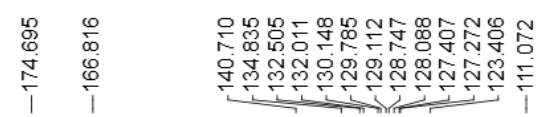

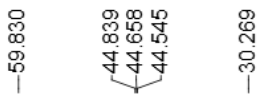

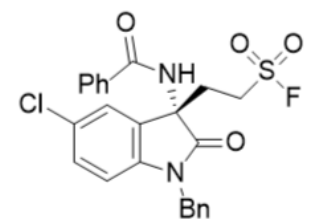

$3 \mathrm{e}$

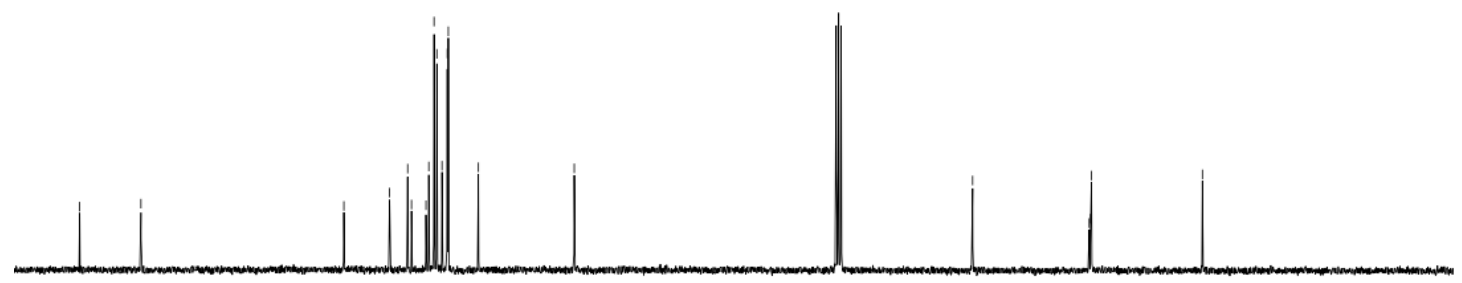

$\begin{array}{lllllllllllllllllll}180 & 170 & 160 & 150 & 140 & 130 & 120 & 110 & 100 & \begin{array}{c}90 \\ \mathrm{f} 1(\mathrm{ppm})\end{array} & 80 & 70 & 60 & 50 & 40 & 30 & 20 & 10 & 0\end{array}$

${ }^{1} \mathrm{H},{ }^{19} \mathrm{~F}$ and ${ }^{13} \mathrm{C}$ NMR spectra of $\mathbf{3 e}$ 


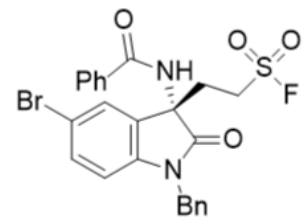

$3 f$

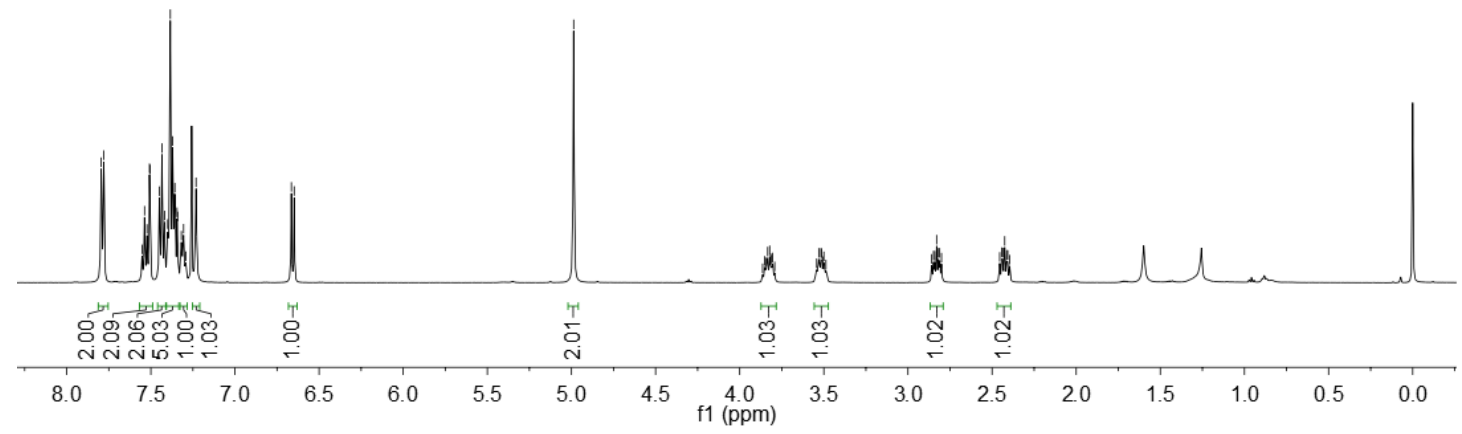

స్ల

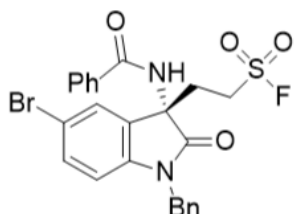

$3 f$

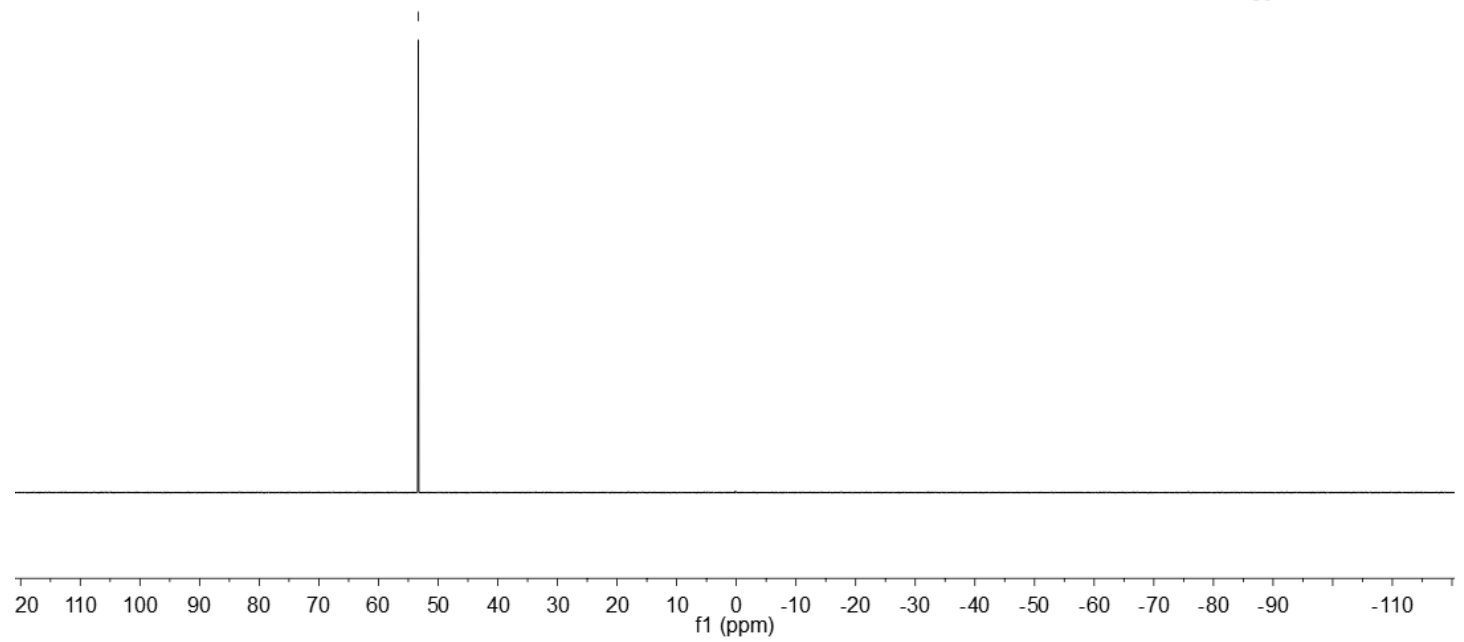




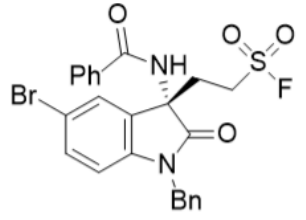

$3 f$
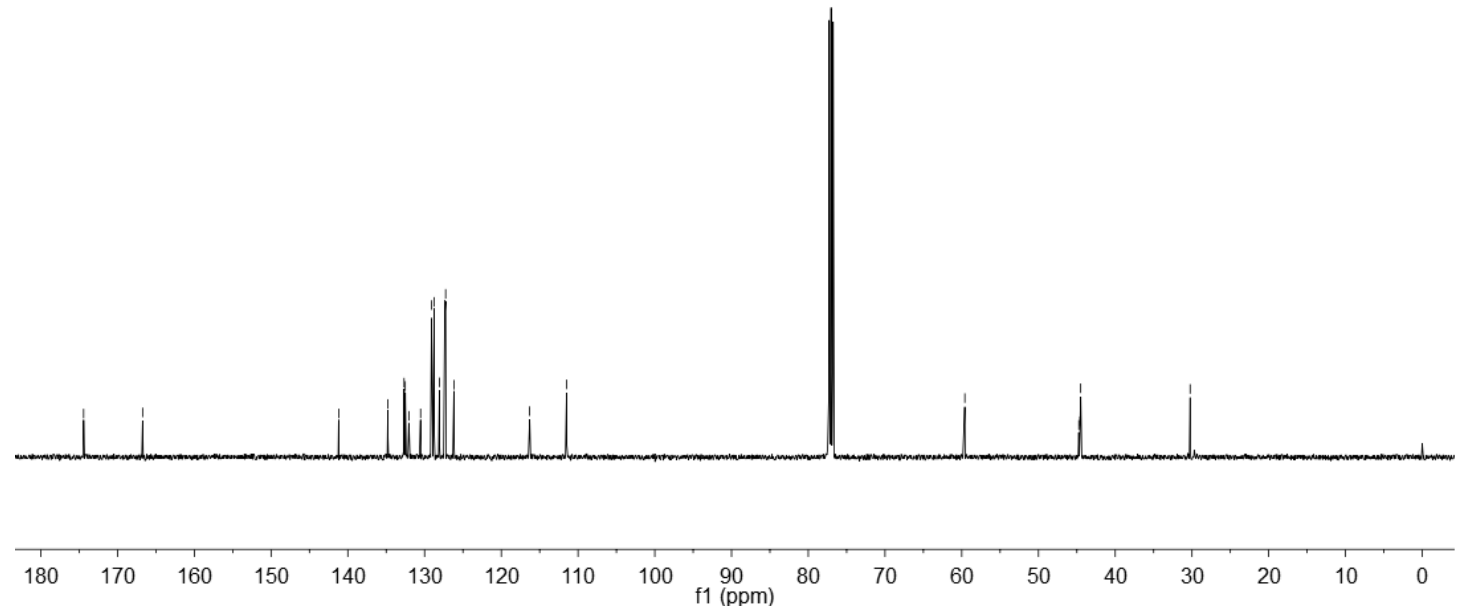

${ }^{1} \mathrm{H},{ }^{19} \mathrm{~F}$ and ${ }^{13} \mathrm{C}$ NMR spectra of $3 \mathbf{f}$

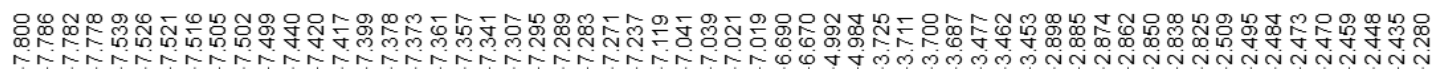

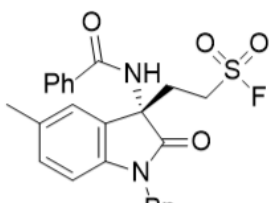

$3 \mathrm{~g}$

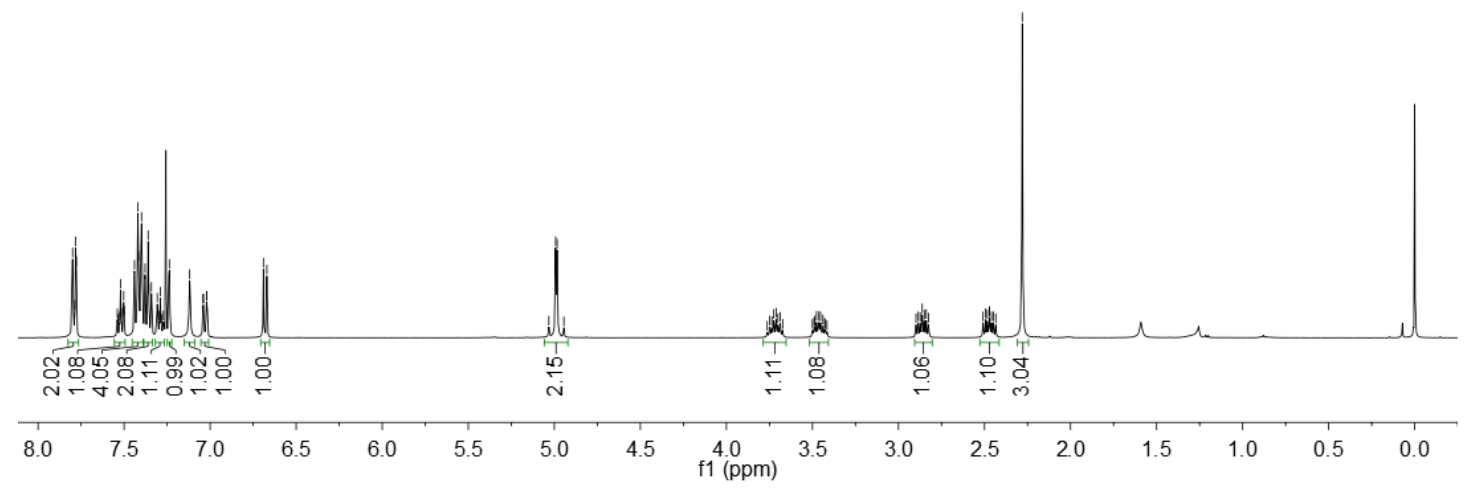




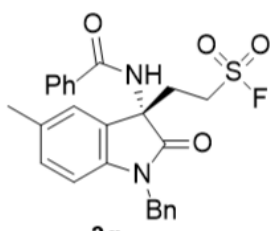

$3 g$

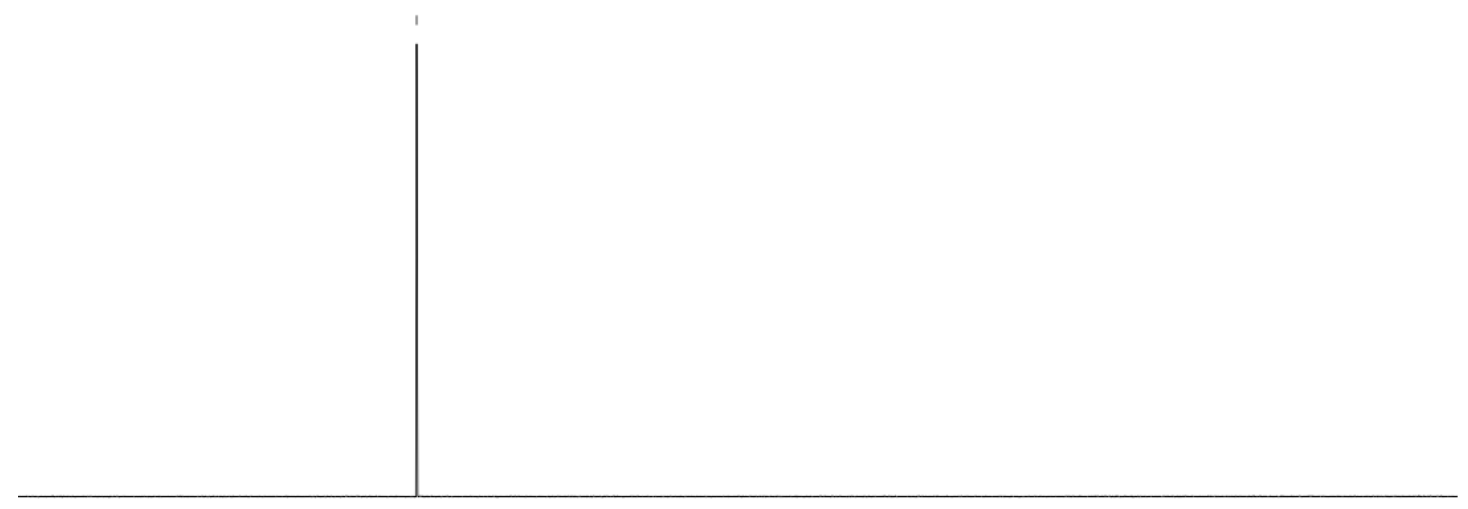

$\begin{array}{lllllllllllllllllllllll}110 & 100 & 90 & 80 & 70 & 60 & 50 & 40 & 30 & 20 & 10 & 0 & 0 & -10 & -20 & -30 & -40 & -50 & -60 & -70 & -80 & -90 & -100\end{array}$

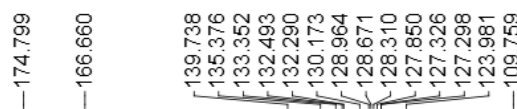

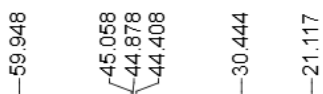

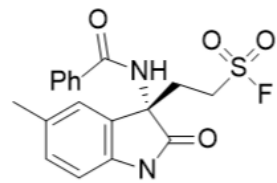

$3 \mathrm{~g}$

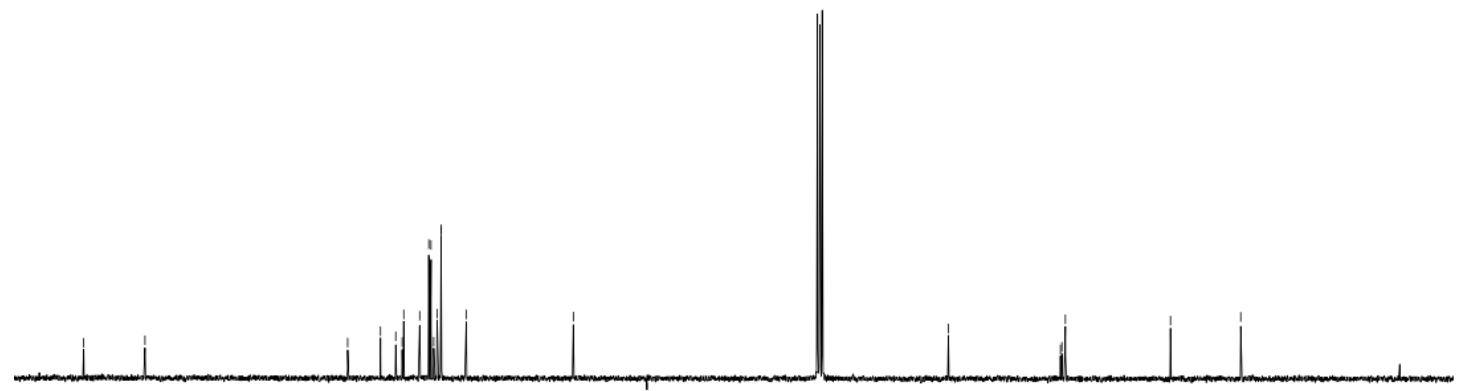

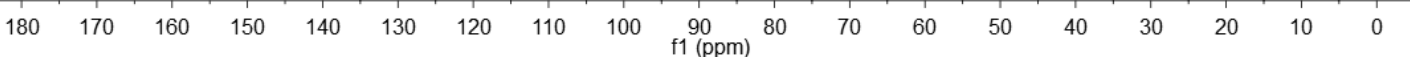

${ }^{1} \mathrm{H},{ }^{19} \mathrm{~F}$ and ${ }^{13} \mathrm{C}$ NMR spectra of $\mathbf{3 g}$ 


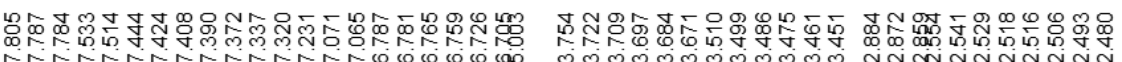

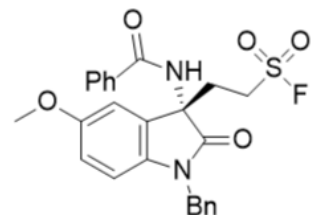

$3 \mathrm{~h}$

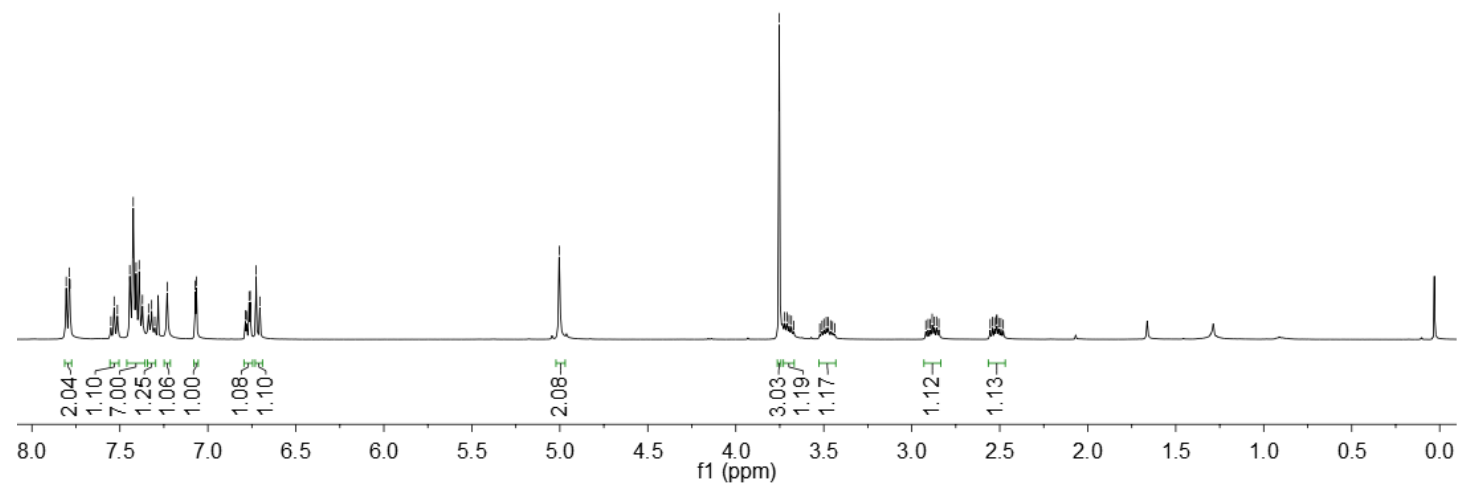

욜

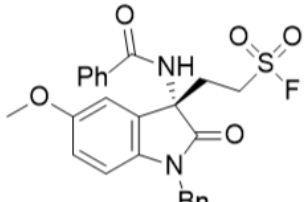

$3 \mathrm{~h}$

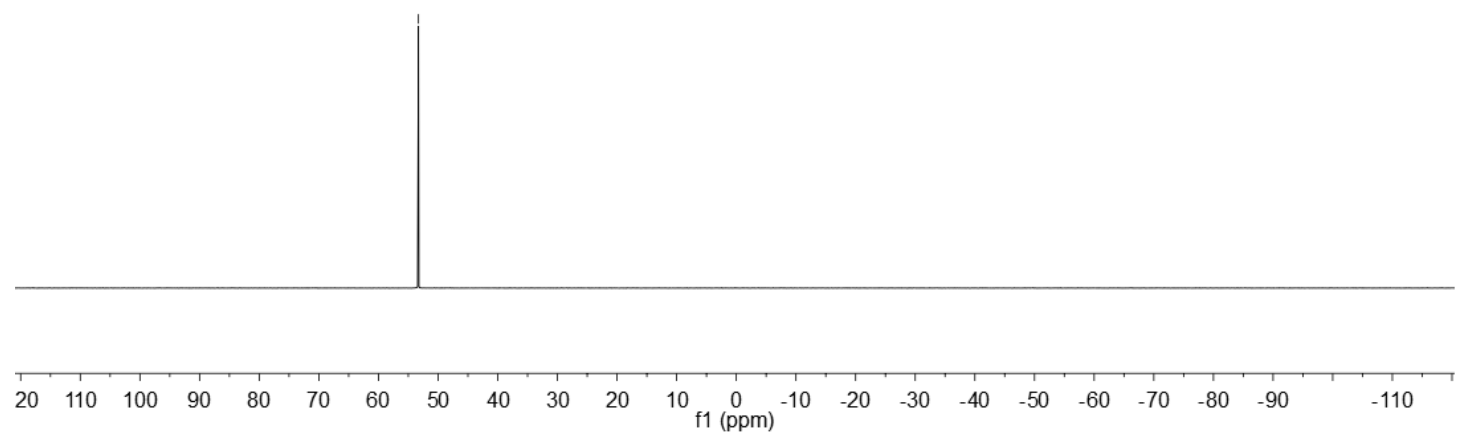




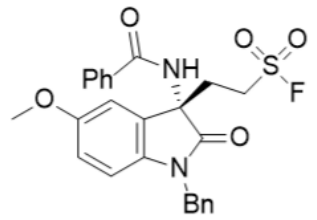

$3 \mathrm{~h}$

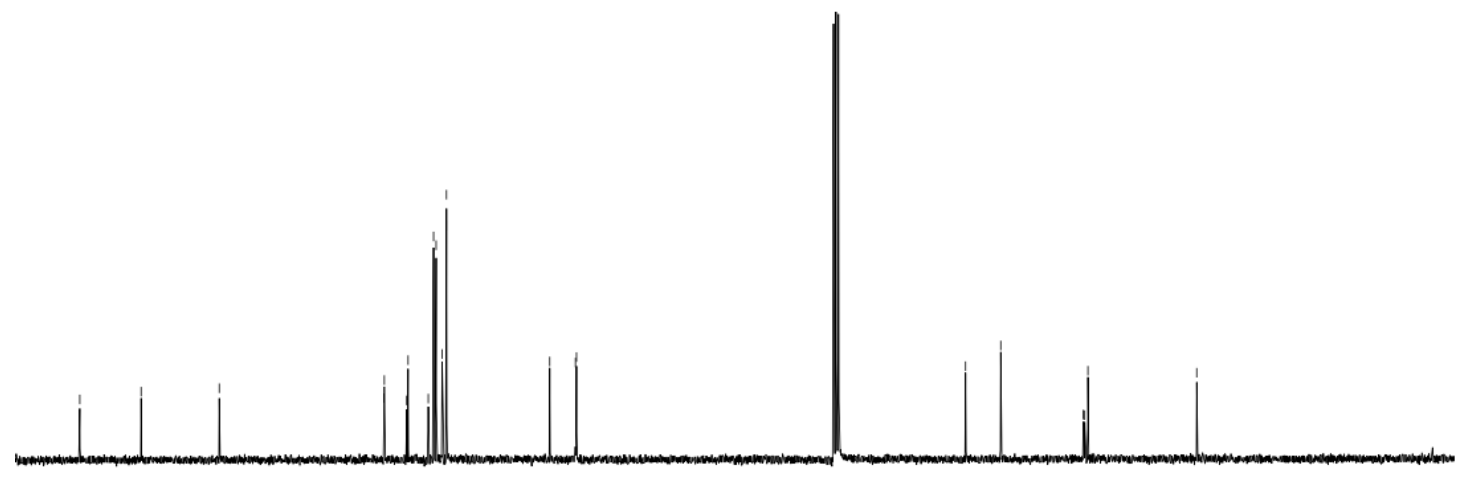

$\begin{array}{lllllllllllllllllllll}180 & 170 & 160 & 150 & 140 & 130 & 120 & 110 & 100 & \underset{\mathrm{f} 1}{90}(\mathrm{ppm}) & 80 & 70 & 60 & 50 & 40 & 30 & 20 & 10 & 0\end{array}$

${ }^{1} \mathrm{H},{ }^{19} \mathrm{~F}$ and ${ }^{13} \mathrm{C}$ NMR spectra of $\mathbf{3 h}$

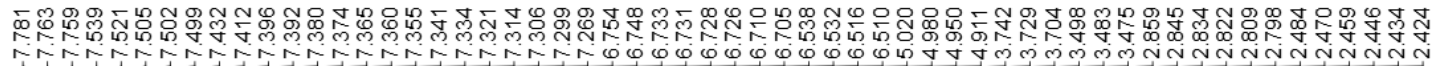
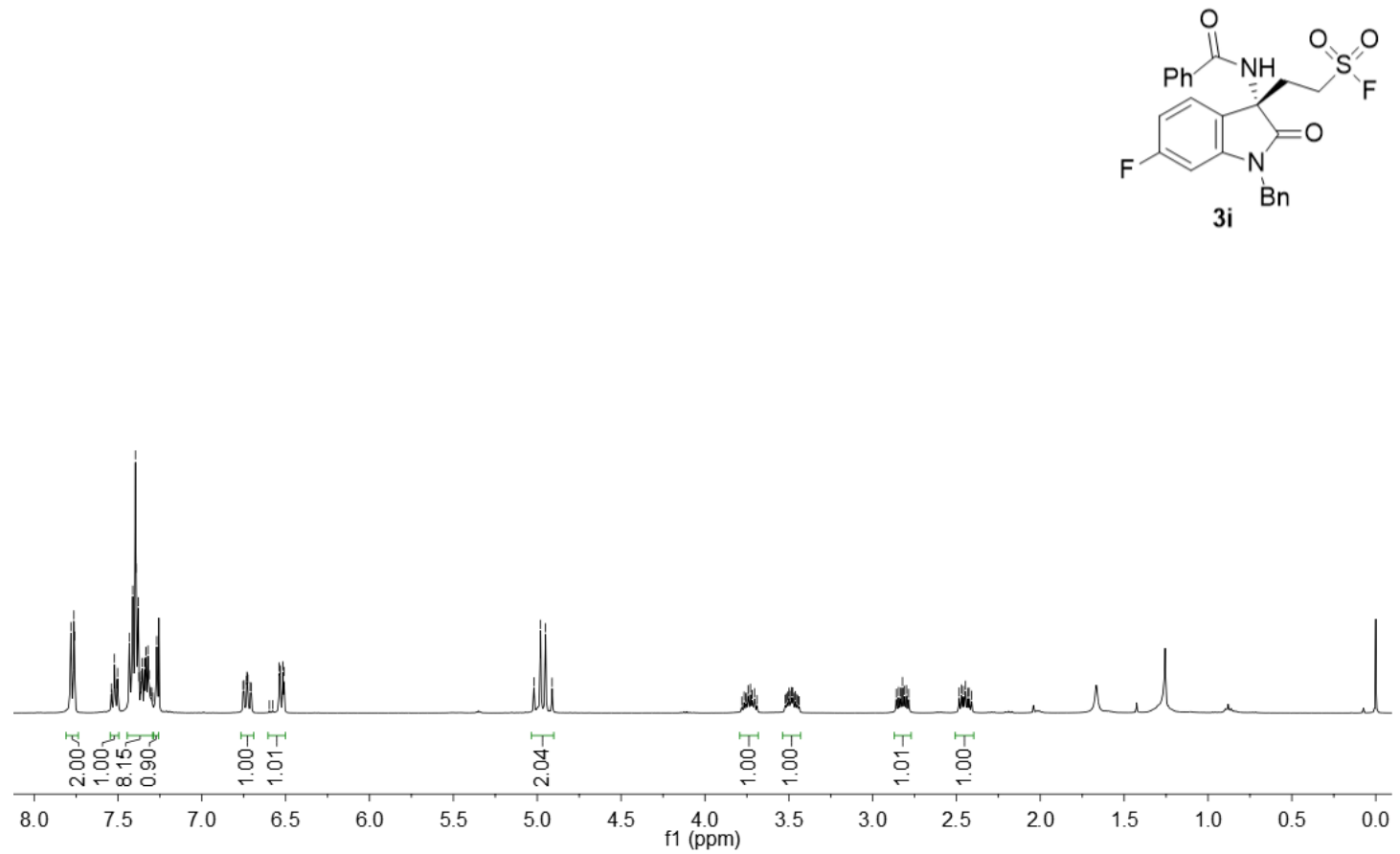


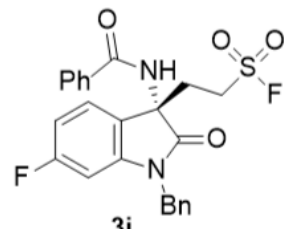

$3 i^{\mathrm{Bn}}$

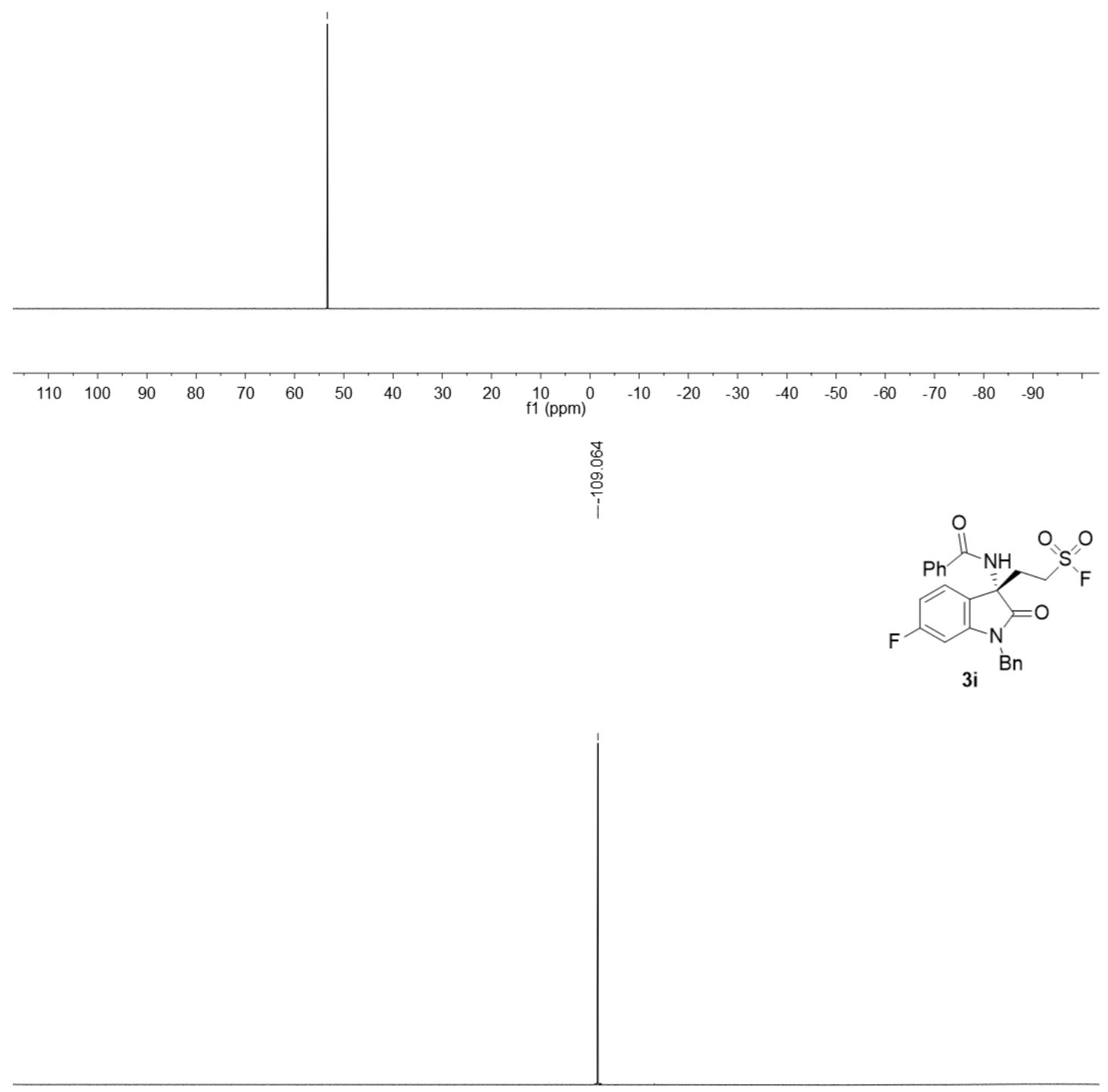

$\begin{array}{lllllllllll}10 & 0 & -10 & -20 & -30 & -40 & -50 & -60 & -70 & -80 & -90 \\ \mathrm{f} 1 & -100\end{array}$ 

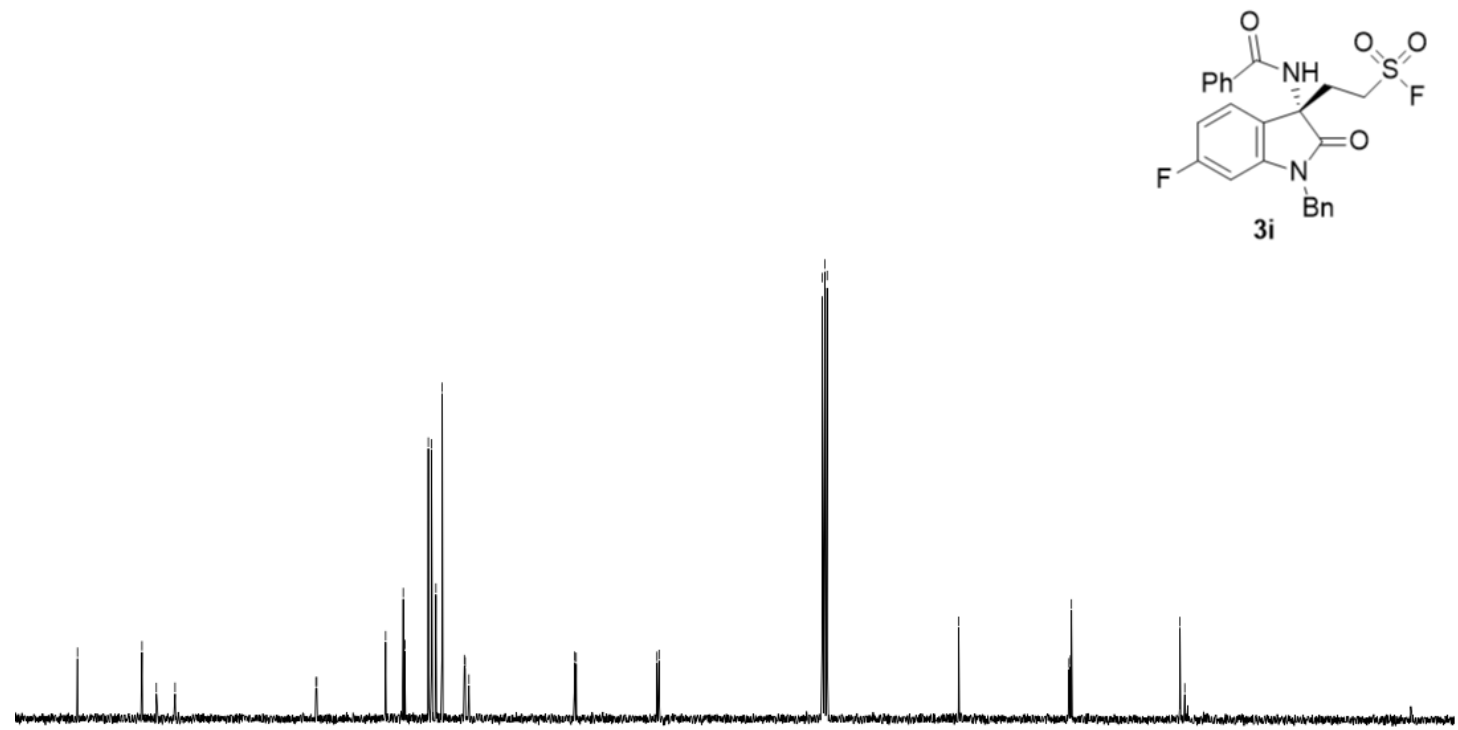

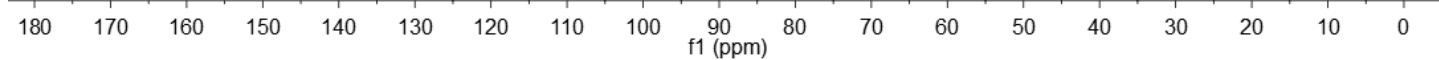

${ }^{1} \mathrm{H},{ }^{19} \mathrm{~F}$ and ${ }^{13} \mathrm{C}$ NMR spectra of $\mathbf{3 i}$

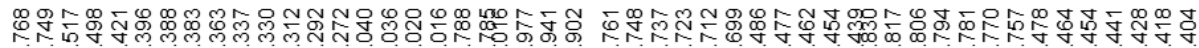

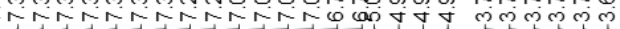

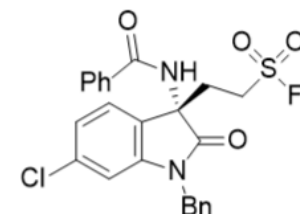

$3 \mathbf{j}$

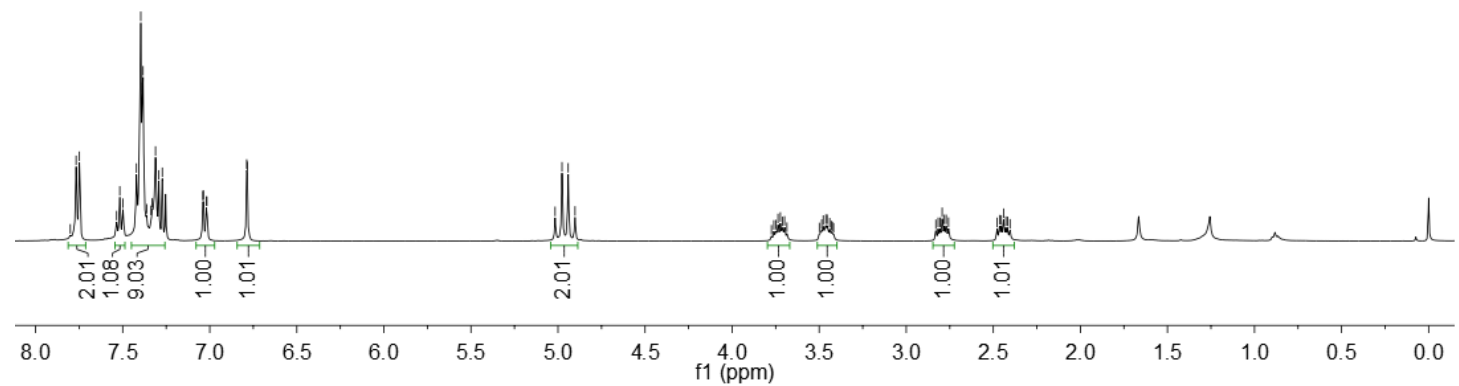



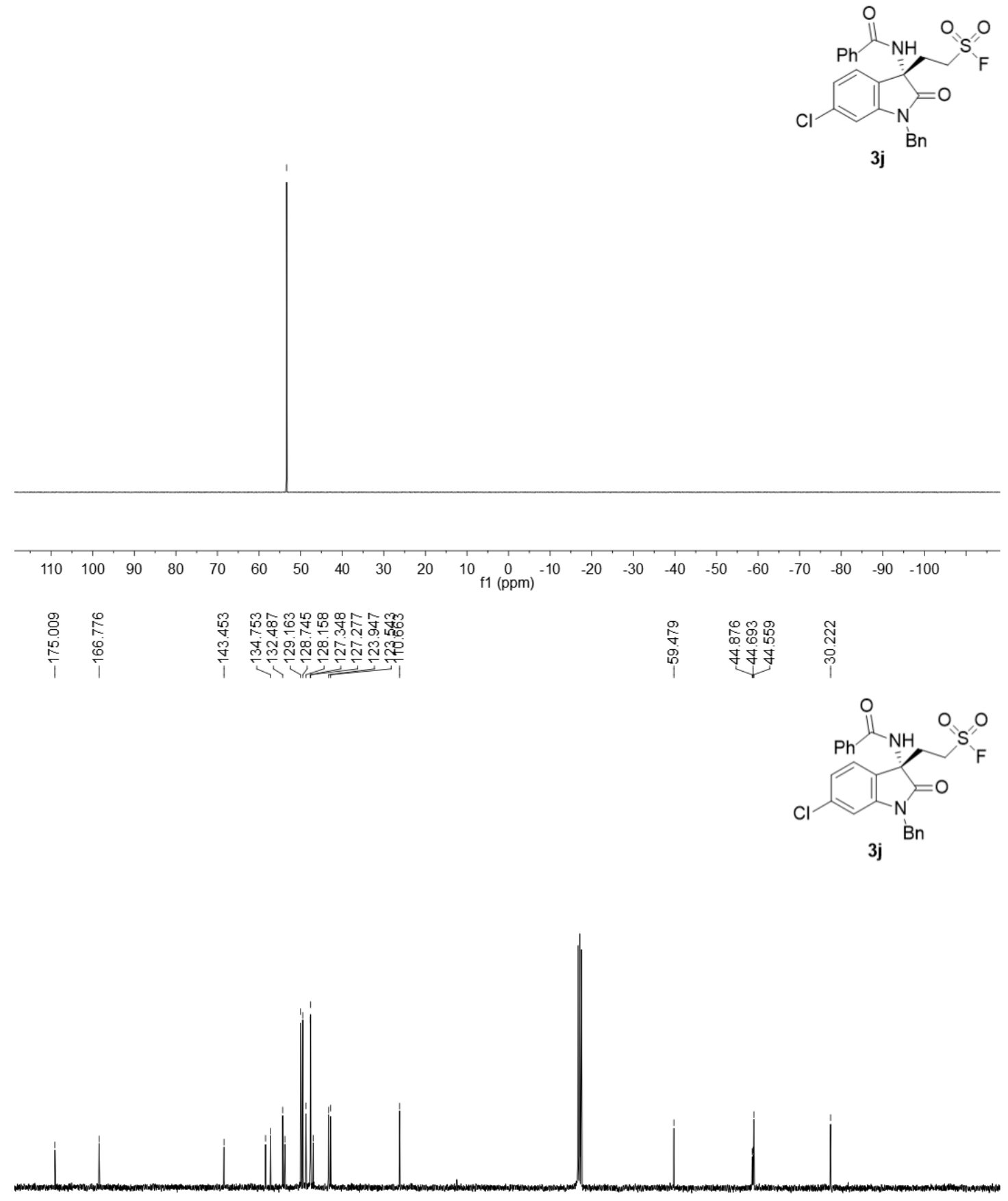

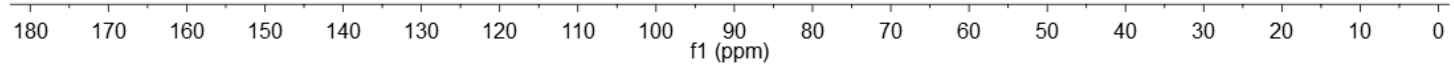

${ }^{1} \mathrm{H},{ }^{19} \mathrm{~F}$ and ${ }^{13} \mathrm{C}$ NMR spectra of $3 \mathbf{j}$ 


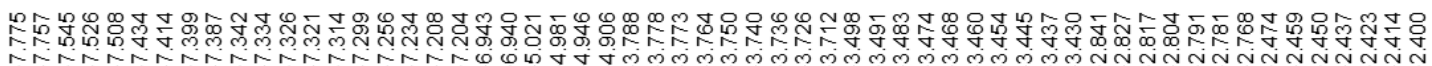

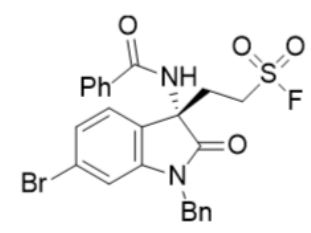

3k

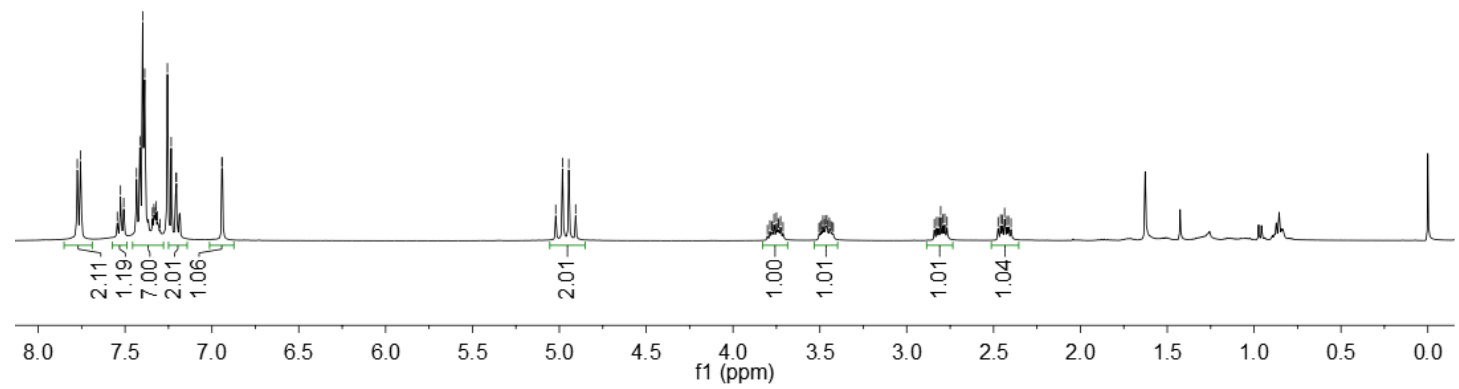

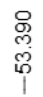

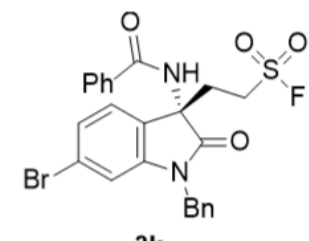

$3 k$

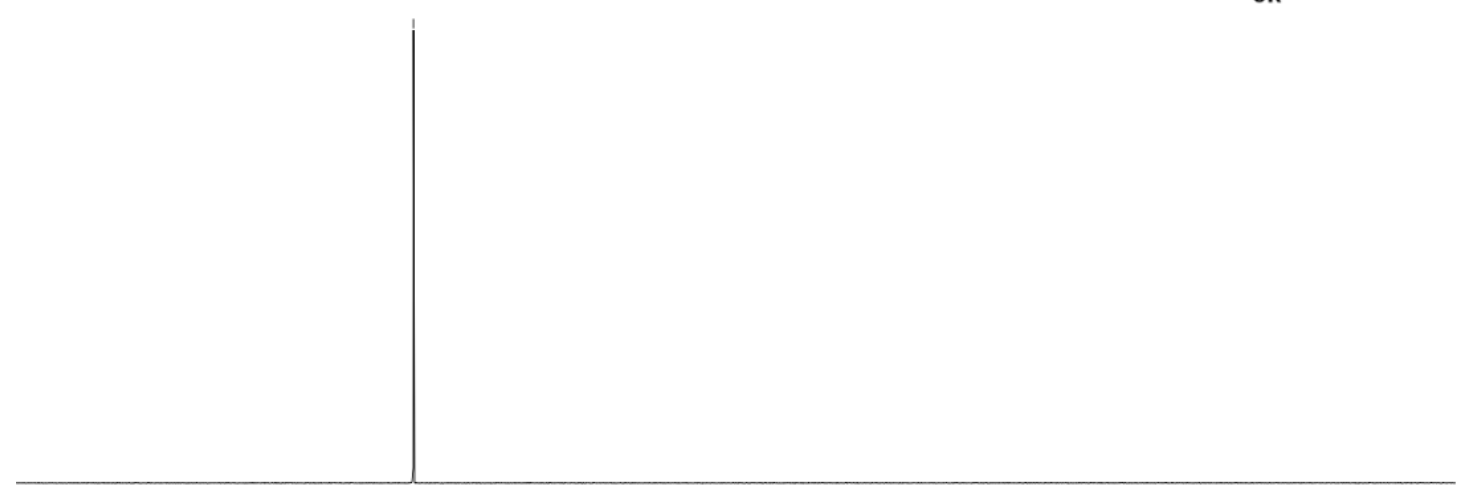

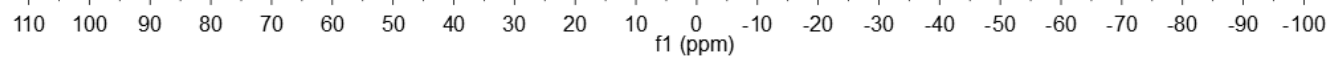




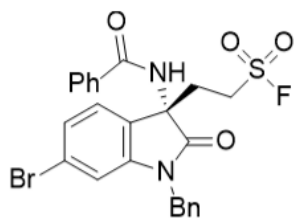

$3 k$

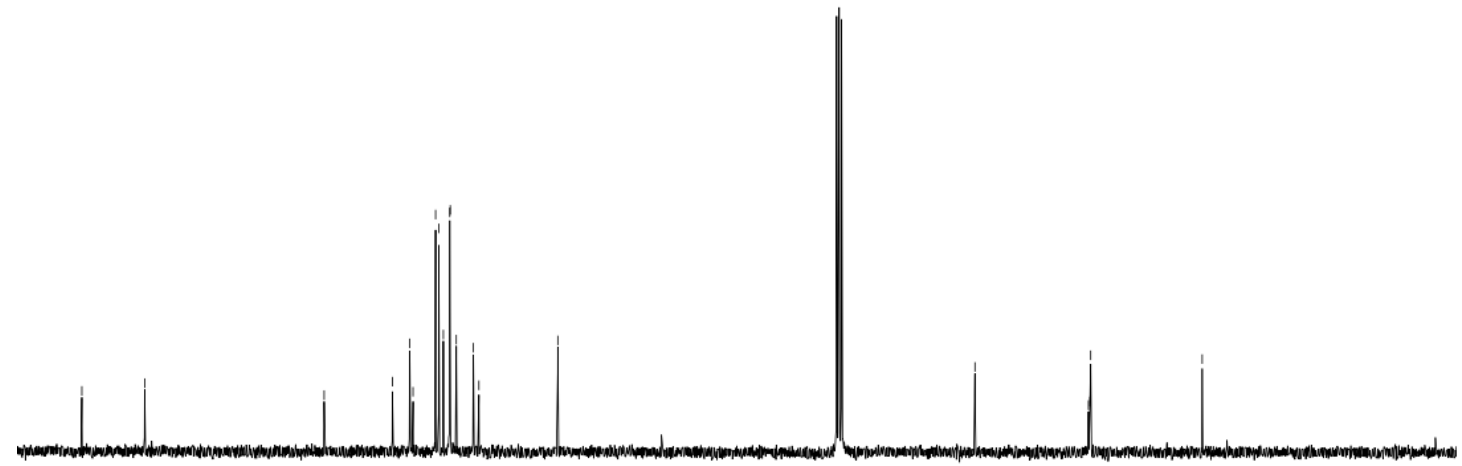

$\begin{array}{llllllllllllllllllll}180 & 170 & 160 & 150 & 140 & 130 & 120 & 110 & 100 & 90 & 80 & 70 & 60 & 50 & 40 & 30 & 20 & 10 & 0\end{array}$

${ }^{1} \mathrm{H},{ }^{19} \mathrm{~F}$ and ${ }^{13} \mathrm{C}$ NMR spectra of $\mathbf{3 k}$

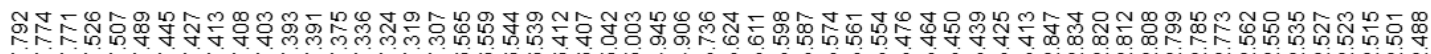
NiN

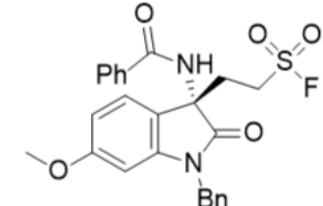

31

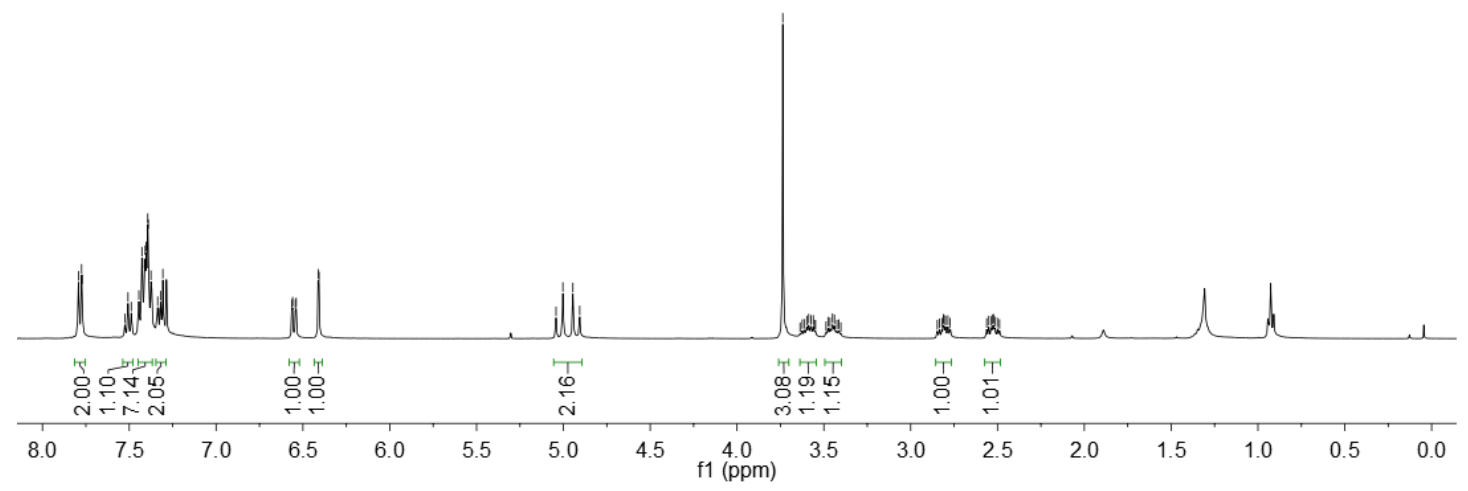




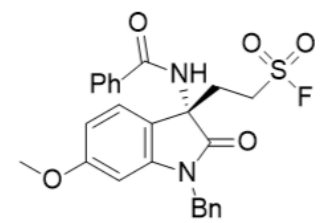

3!

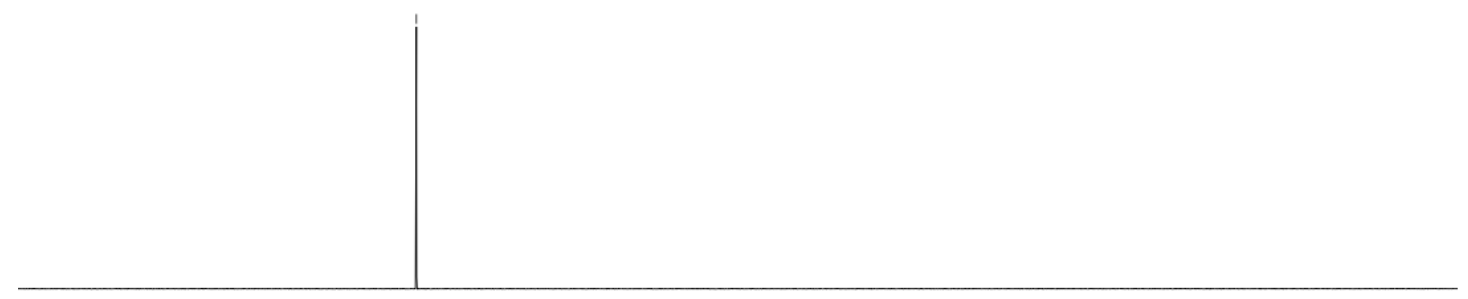

$\begin{array}{lllllllllllllllllllllllll}110 & 100 & 90 & 80 & 70 & 60 & 50 & 40 & 30 & 20 & 10 & 0 & -10 & -20 & -30 & -40 & -50 & -60 & -70 & -80 & -90 & -100 & \end{array}$

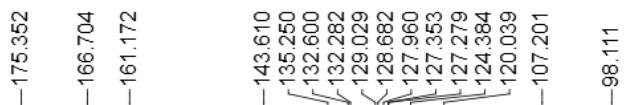

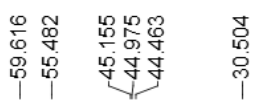

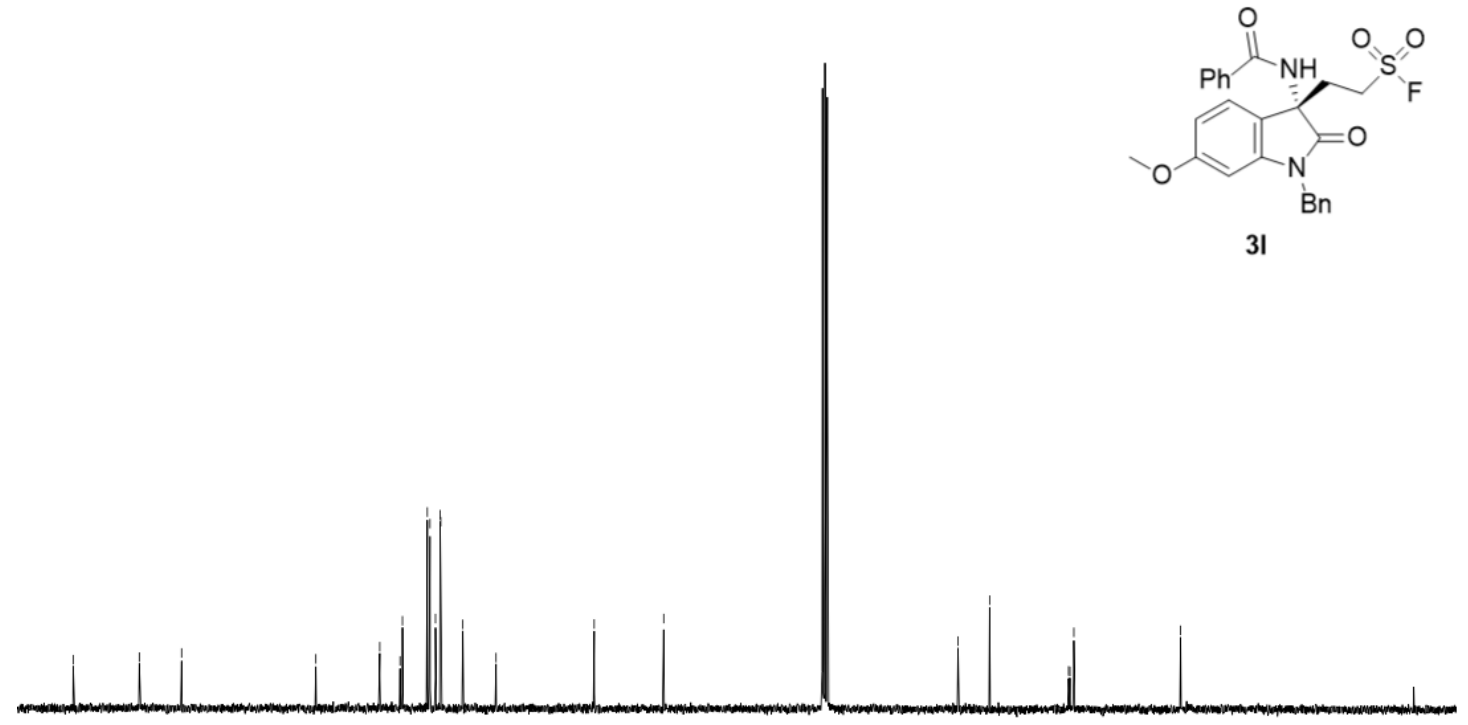

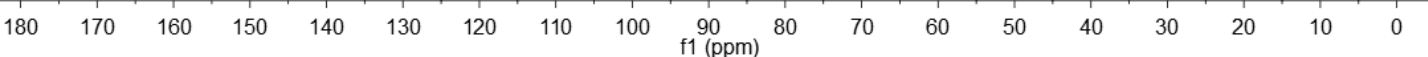

${ }^{1} \mathrm{H},{ }^{19} \mathrm{~F}$ and ${ }^{13} \mathrm{C}$ NMR spectra of $3 \mathbf{l}$ 


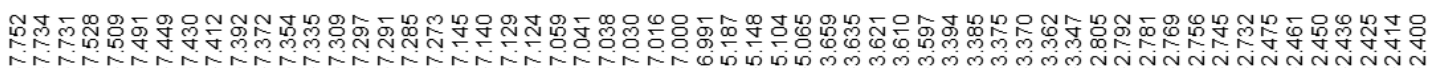
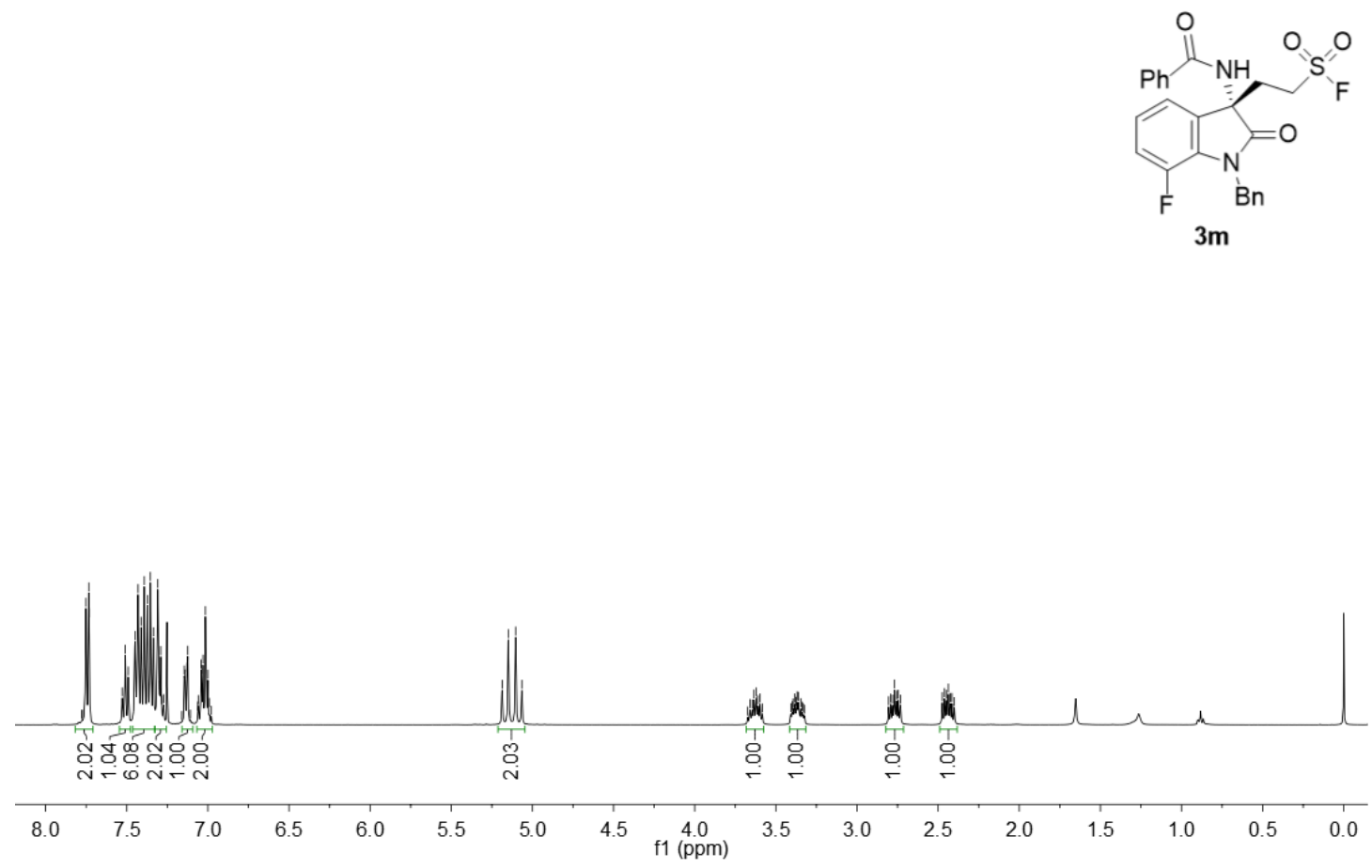

$\stackrel{\substack{m \\ \stackrel{n}{i}}}{i}$

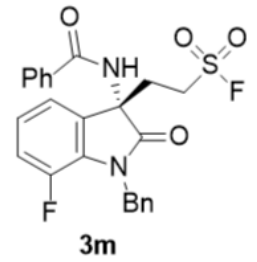

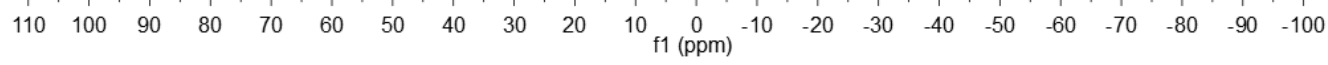



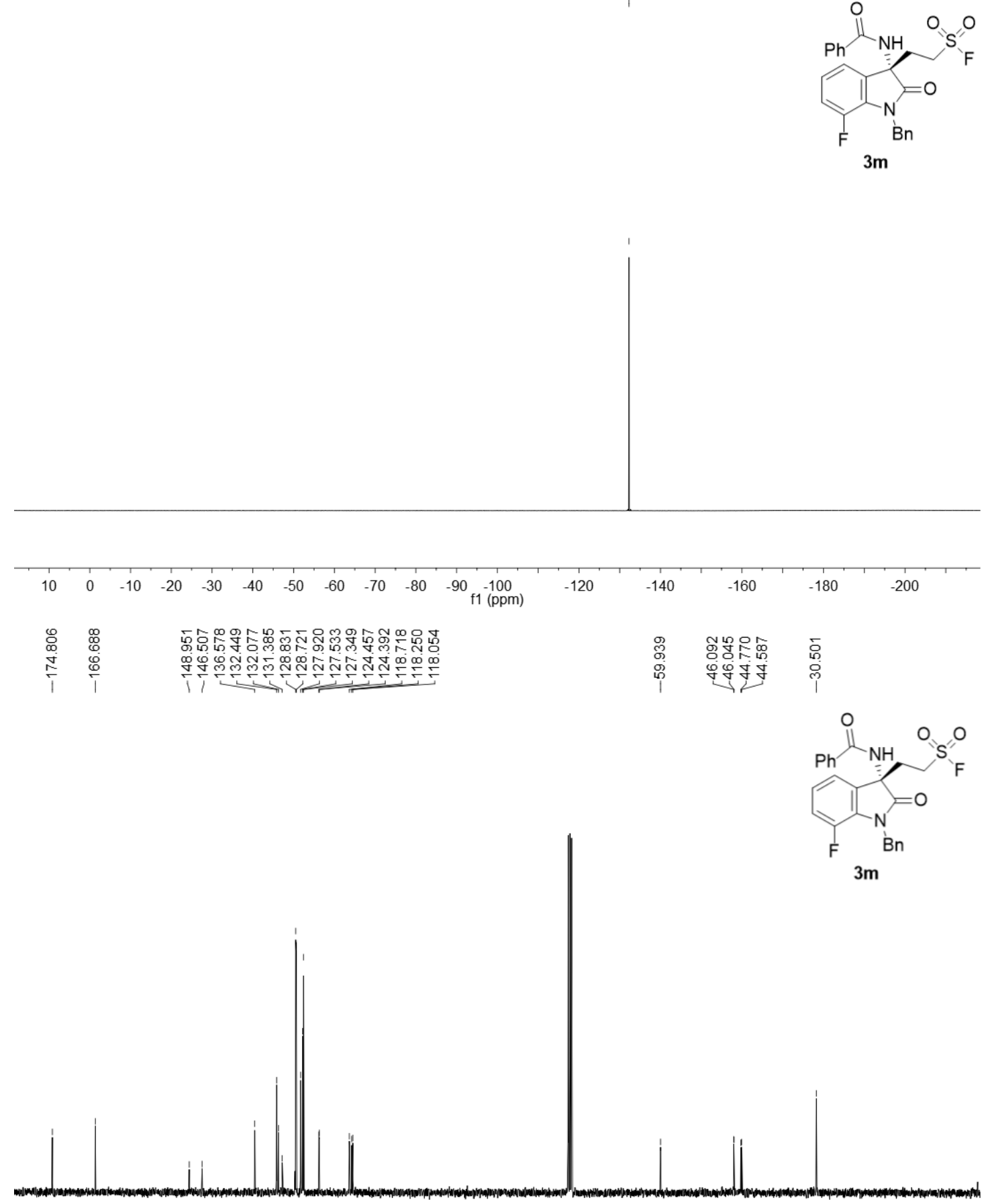

\begin{tabular}{lllllllllllllllllll}
\hline 180 & 170 & 160 & 150 & 140 & 130 & 120 & 110 & 100 & 90 & 90 & 70 & 60 & 50 & 40 & 30 & 20 & 10 & 0
\end{tabular}

${ }^{1} \mathrm{H},{ }^{19} \mathrm{~F}$ and ${ }^{13} \mathrm{C}$ NMR spectra of $3 \mathbf{m}$ 


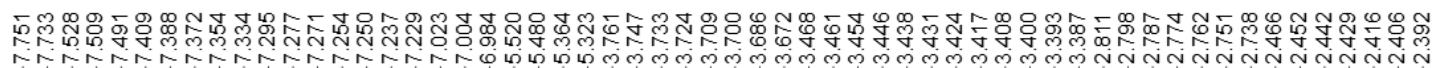

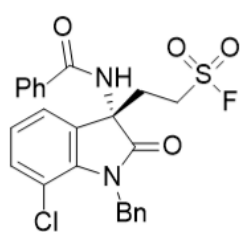

$3 n$

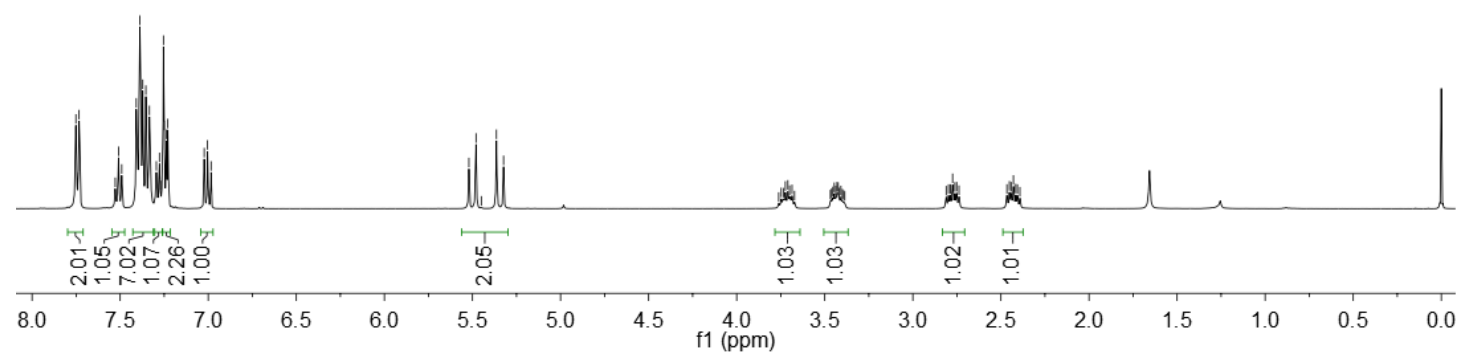

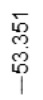

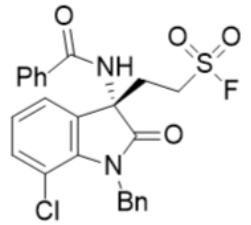

$3 n$

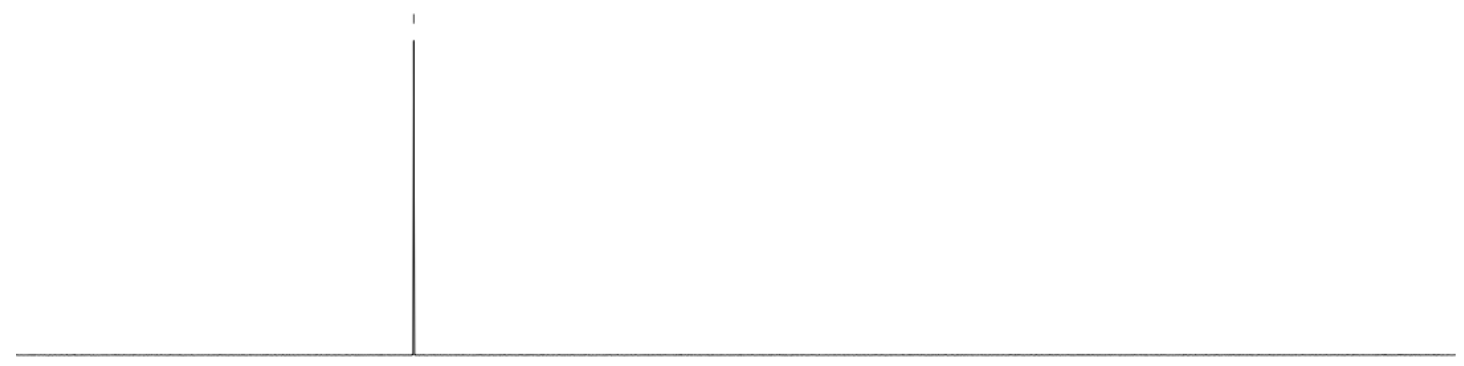

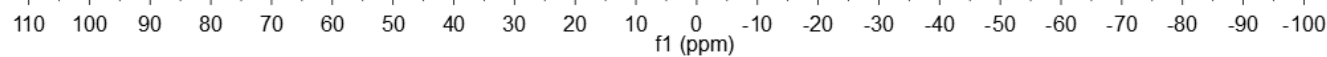




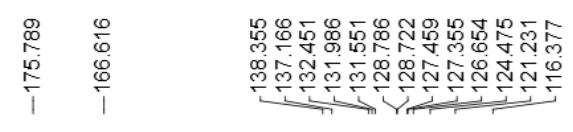

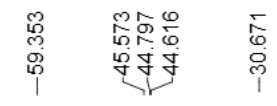

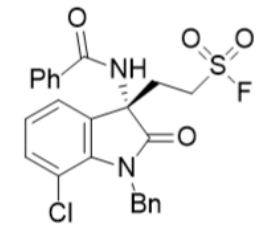

$3 n$
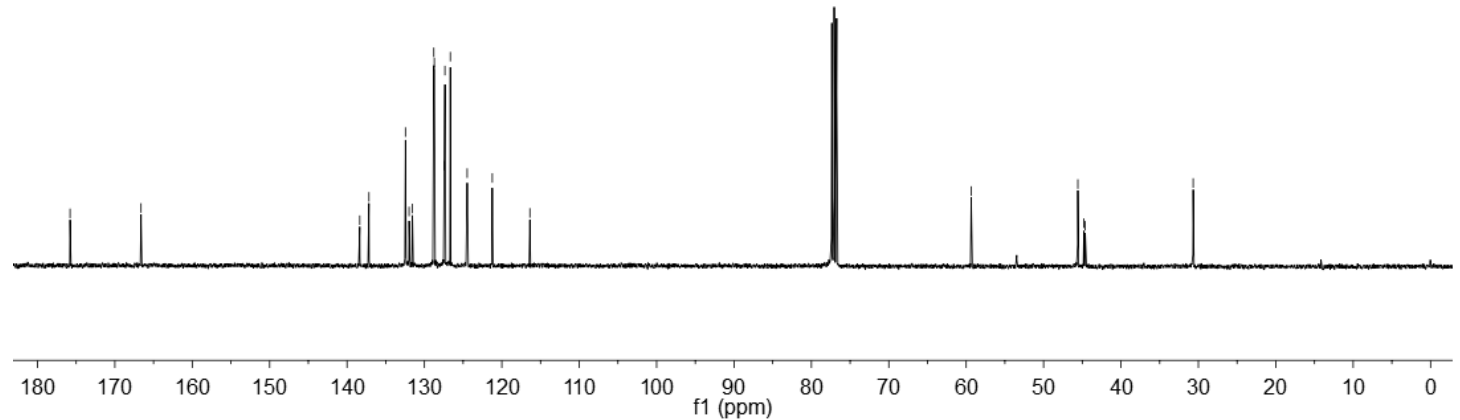

${ }^{1} \mathrm{H},{ }^{19} \mathrm{~F}$ and ${ }^{13} \mathrm{C}$ NMR spectra of $\mathbf{3 n}$

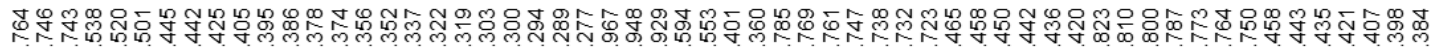

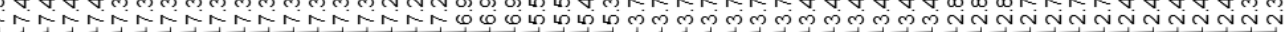<smiles>O=C(NC1(CCS(=O)(=O)F)C(=O)N(Cc2ccccc2)c2c(Br)cccc21)c1ccccc1</smiles>

3o

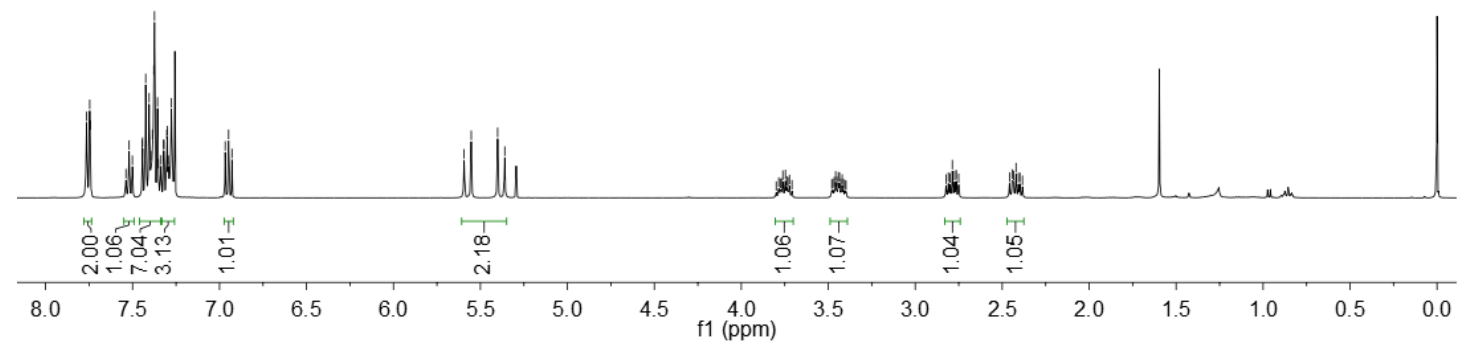




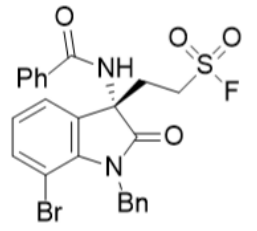

30

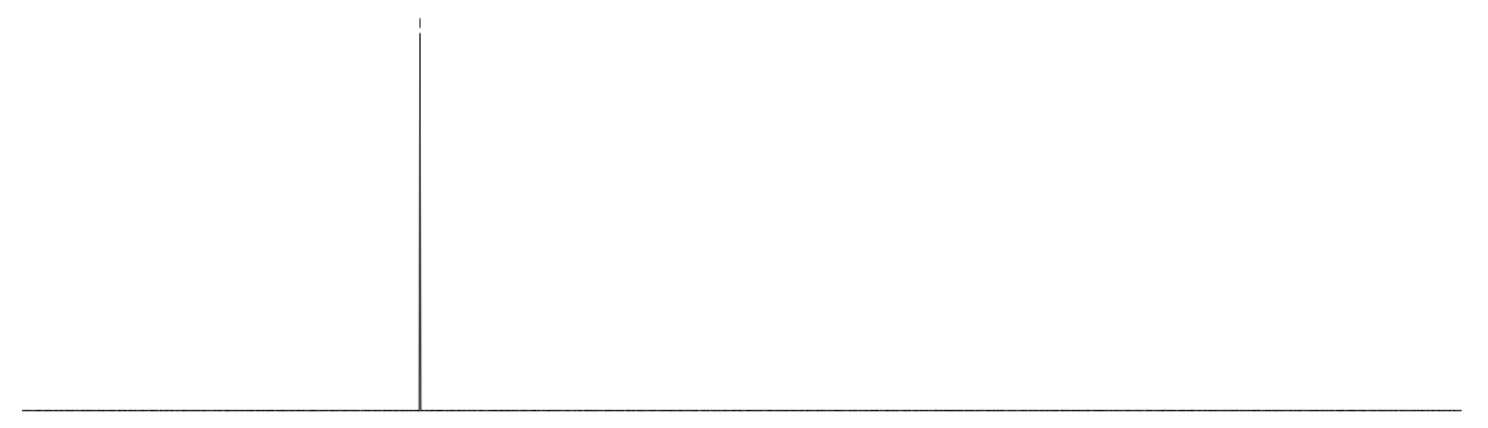

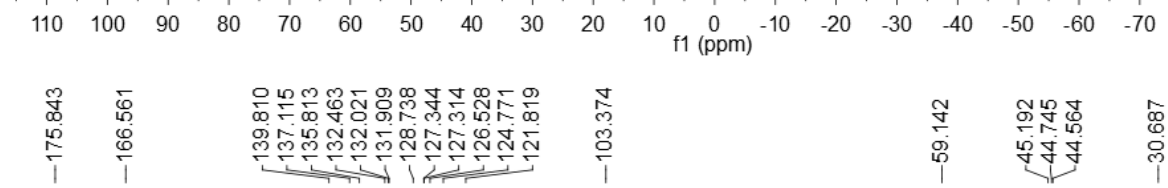

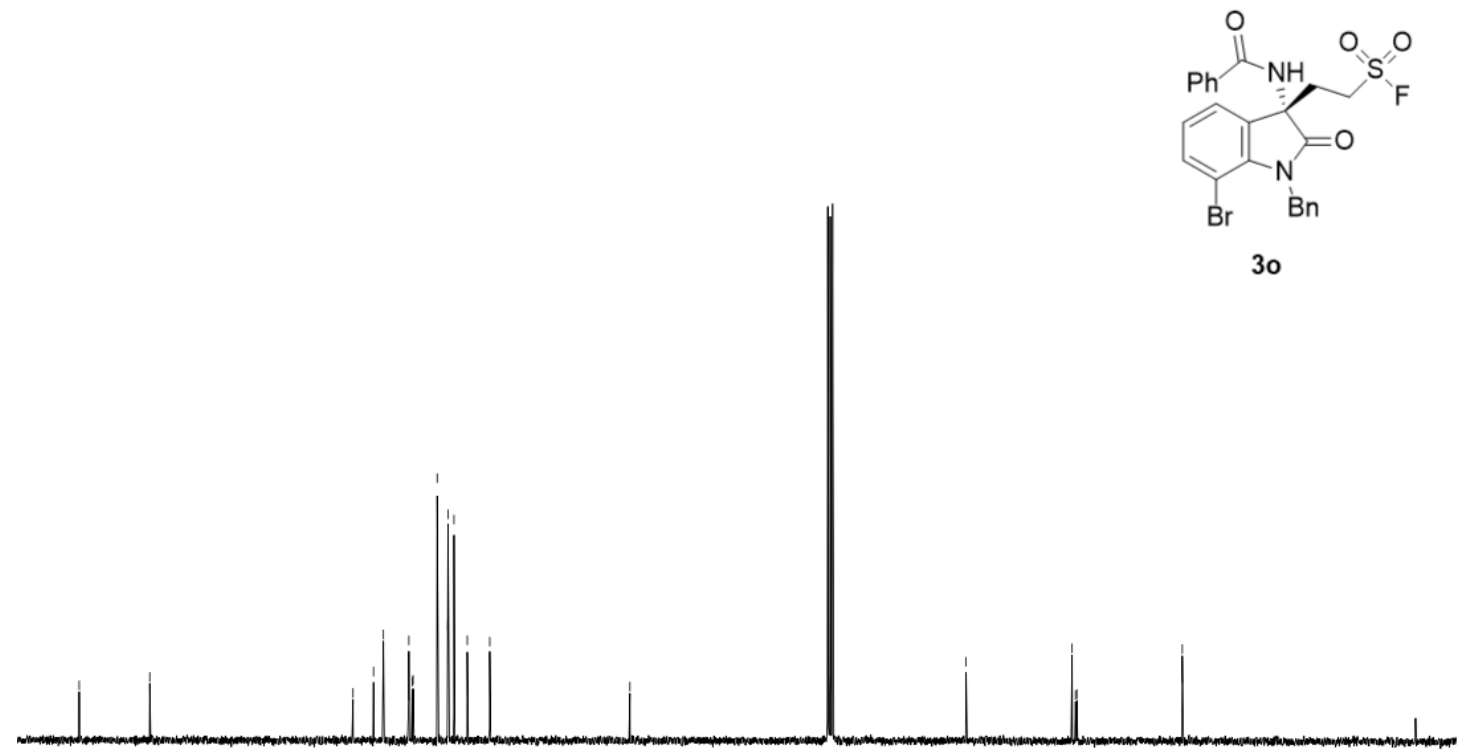

\begin{tabular}{lllllllllllllllllll}
\hline 180 & 170 & 160 & 150 & 140 & 130 & 120 & 110 & 100 & $\begin{array}{c}190 \\
\mathrm{f} 1(\mathrm{ppm})\end{array}$ & 80 & 70 & 60 & 50 & 40 & 30 & 20 & 10 & 0
\end{tabular}

${ }^{1} \mathrm{H},{ }^{19} \mathrm{~F}$ and ${ }^{13} \mathrm{C}$ NMR spectra of $3 \mathbf{0}$ 


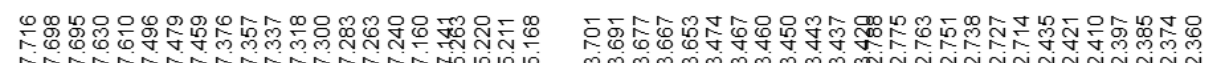

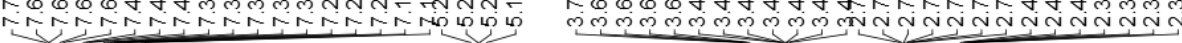

(N)

$3 p$

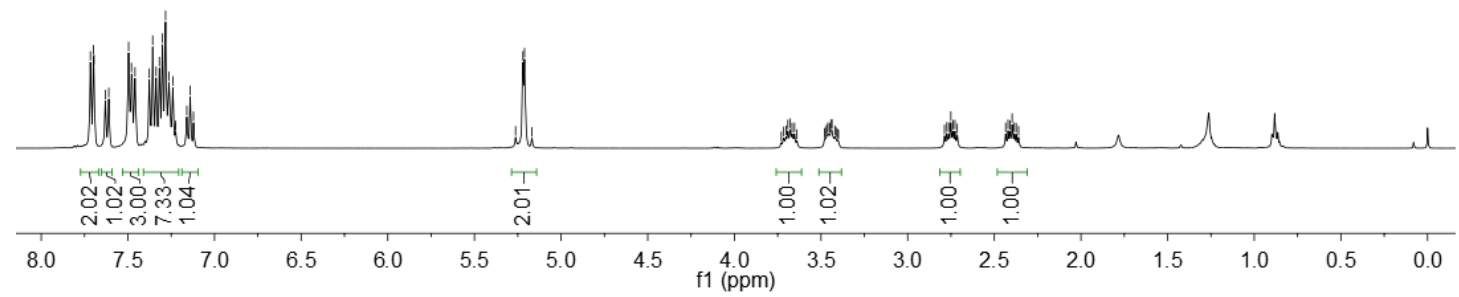

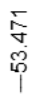

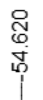

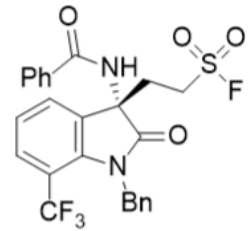

$3 p$

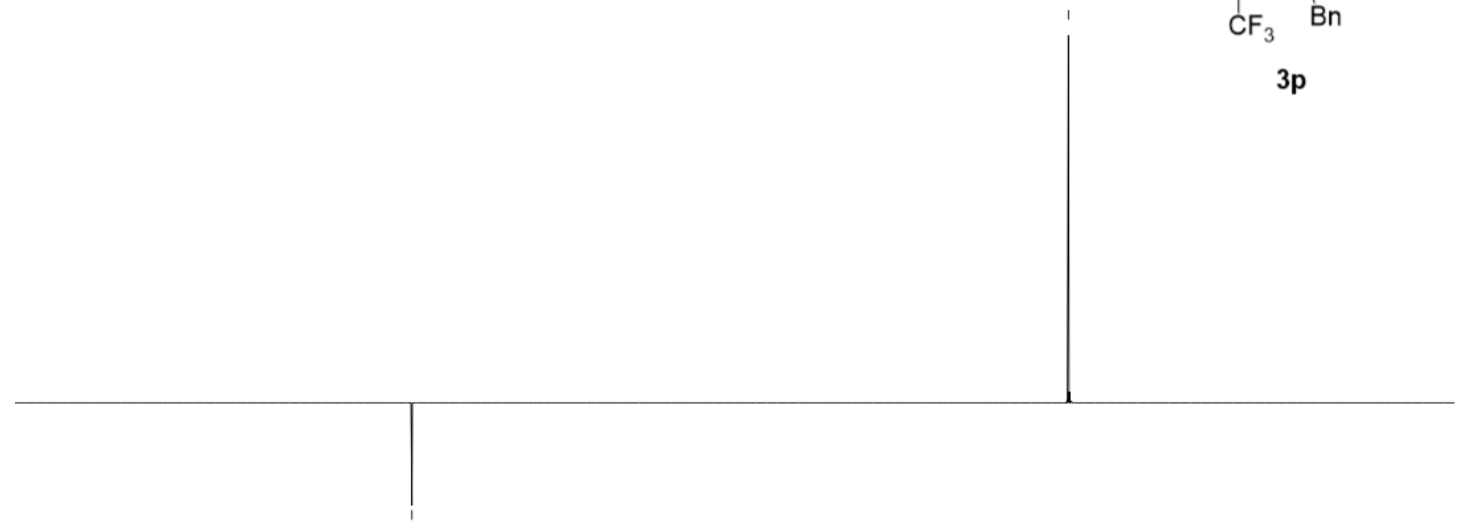

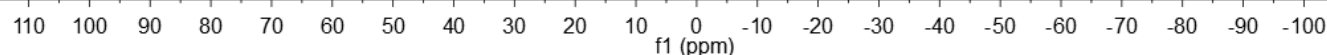




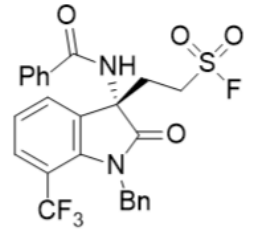

$3 p$
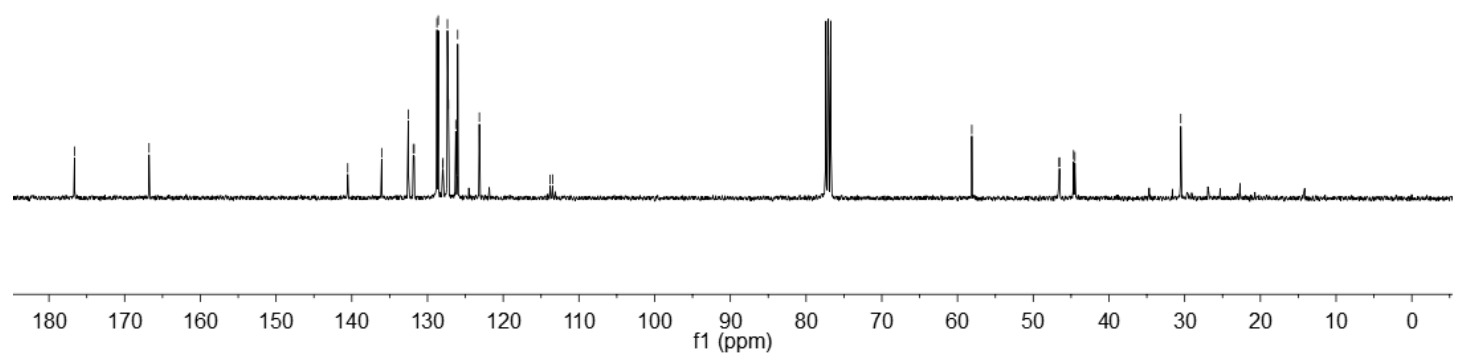

${ }^{1} \mathrm{H},{ }^{19} \mathrm{~F}$ and ${ }^{13} \mathrm{C}$ NMR spectra of $\mathbf{3 p}$

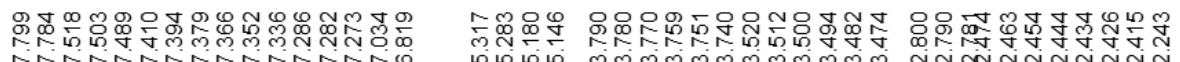

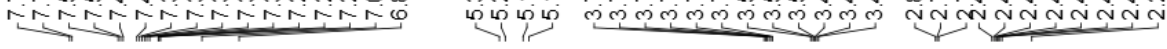

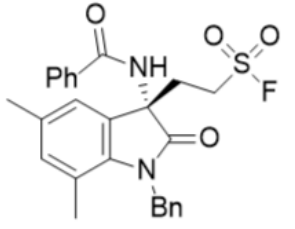

$3 q$

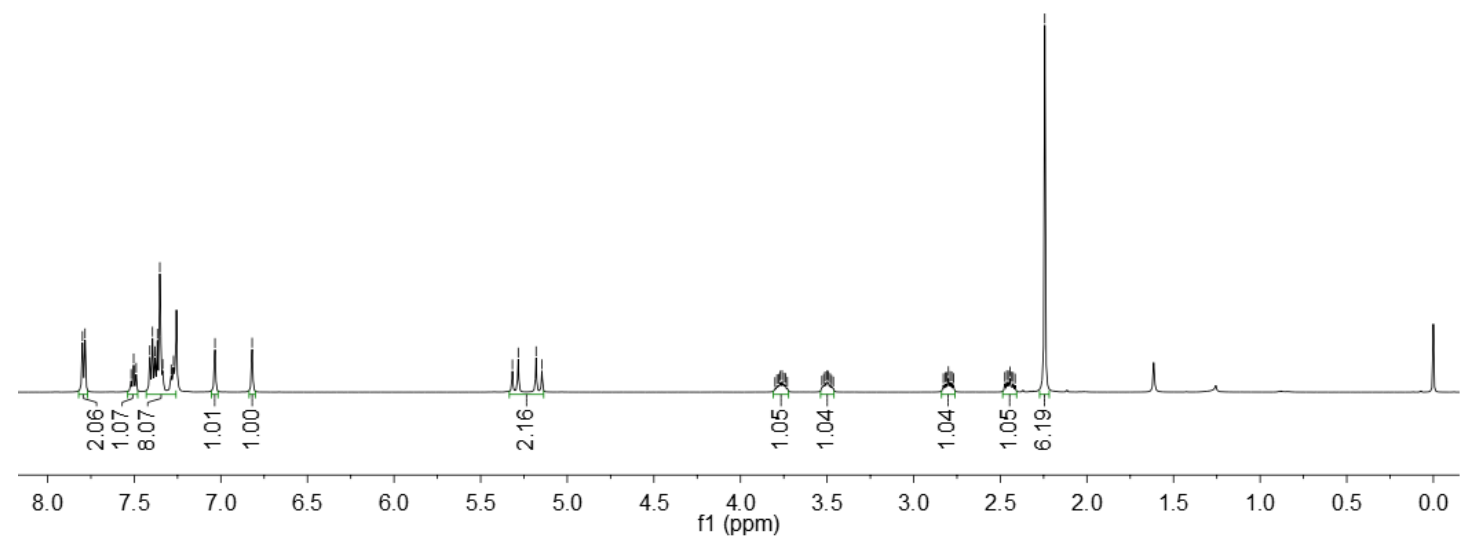




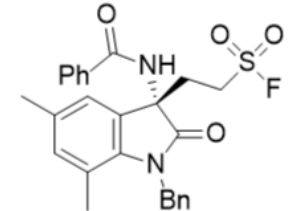

$3 q$

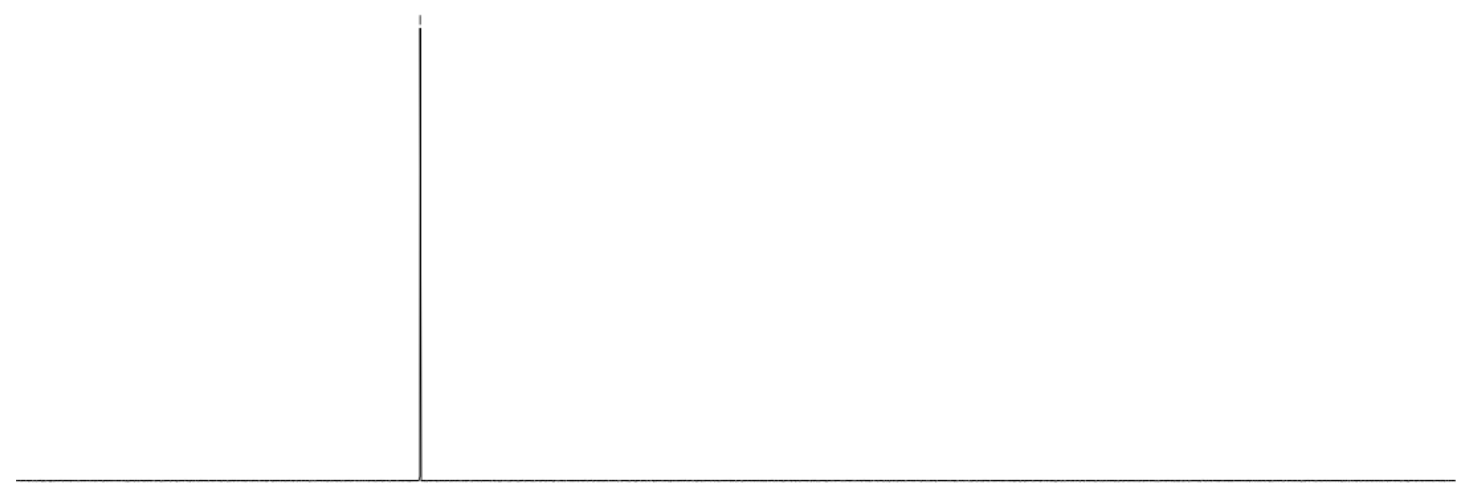

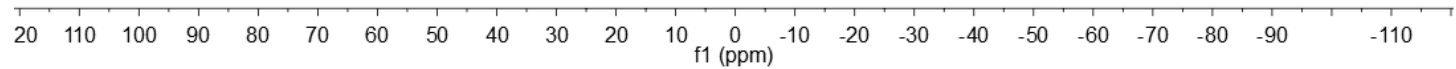

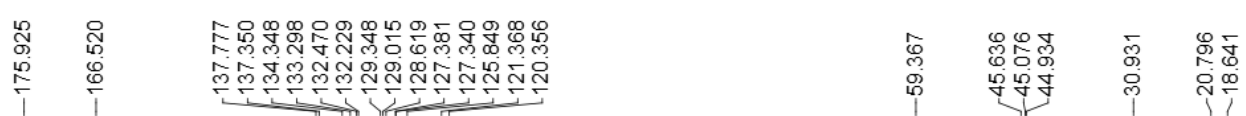

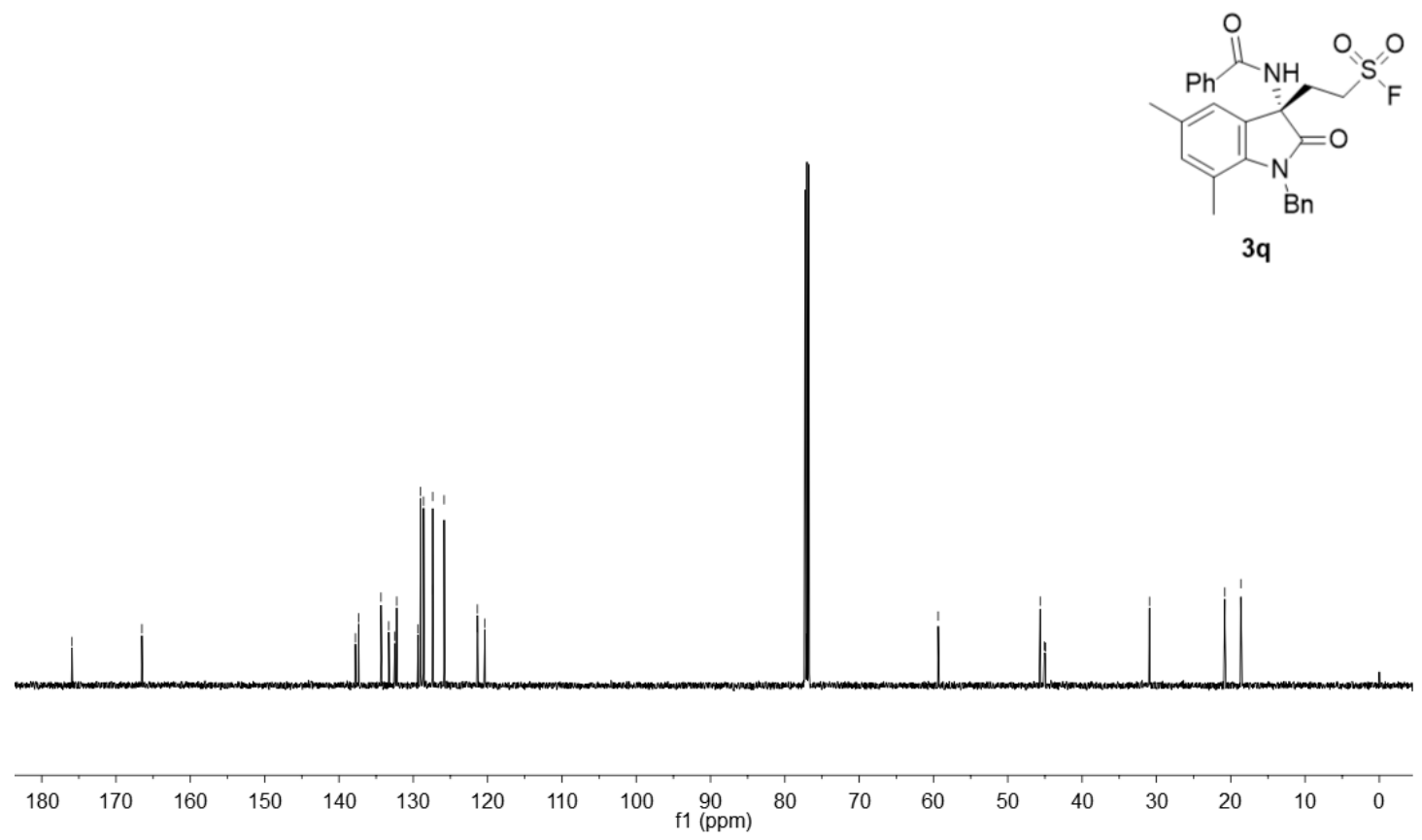

${ }^{1} \mathrm{H},{ }^{19} \mathrm{~F}$ and ${ }^{13} \mathrm{C}$ NMR spectra of $\mathbf{3 q}$ 


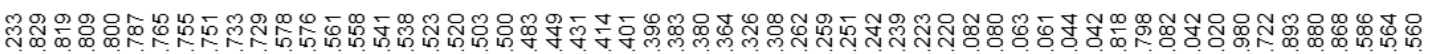

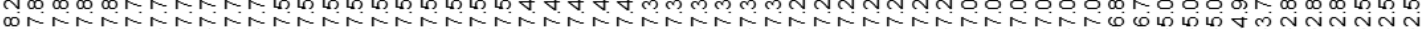
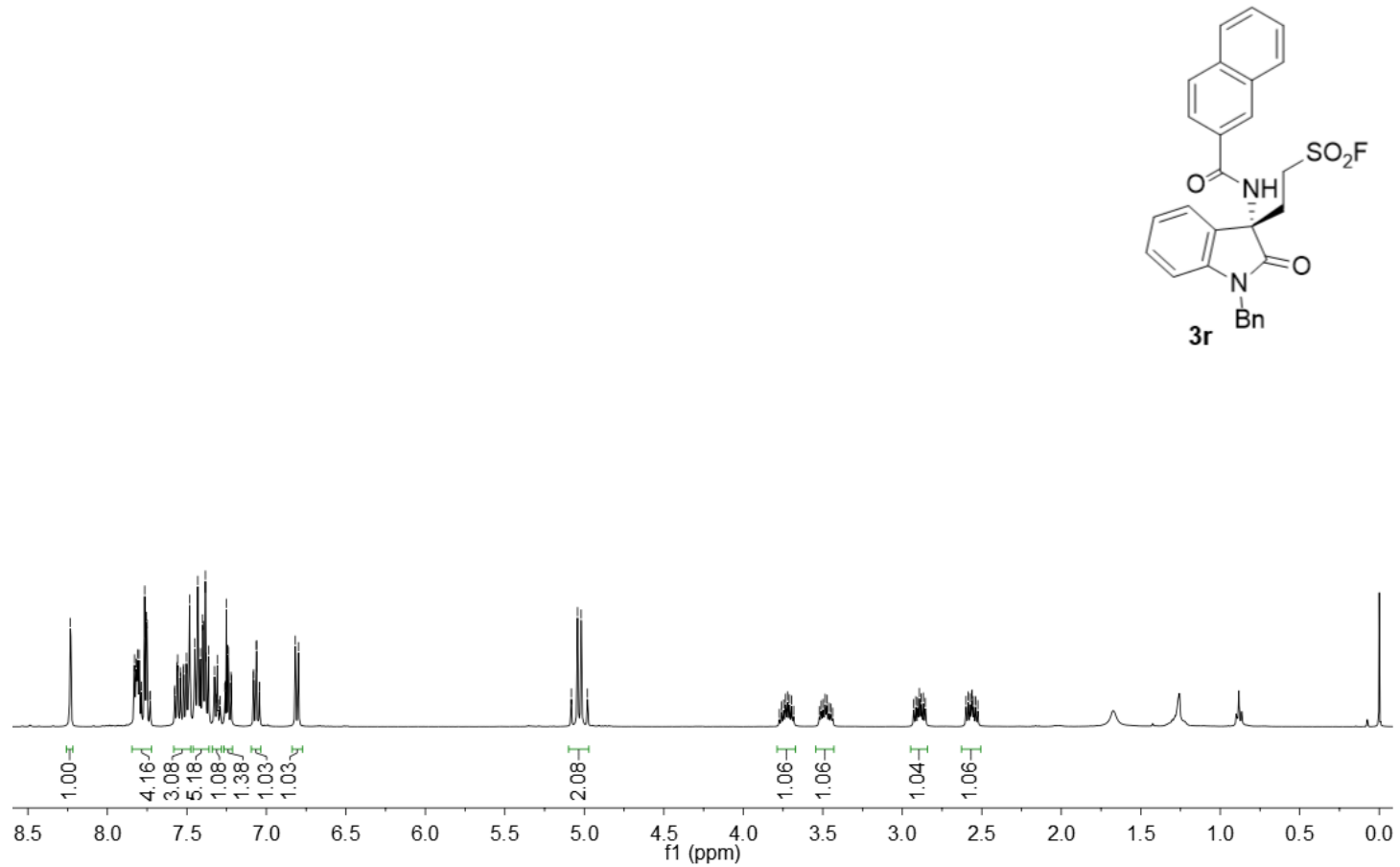

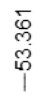

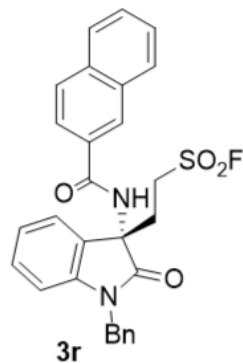

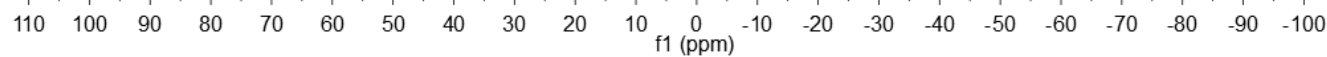



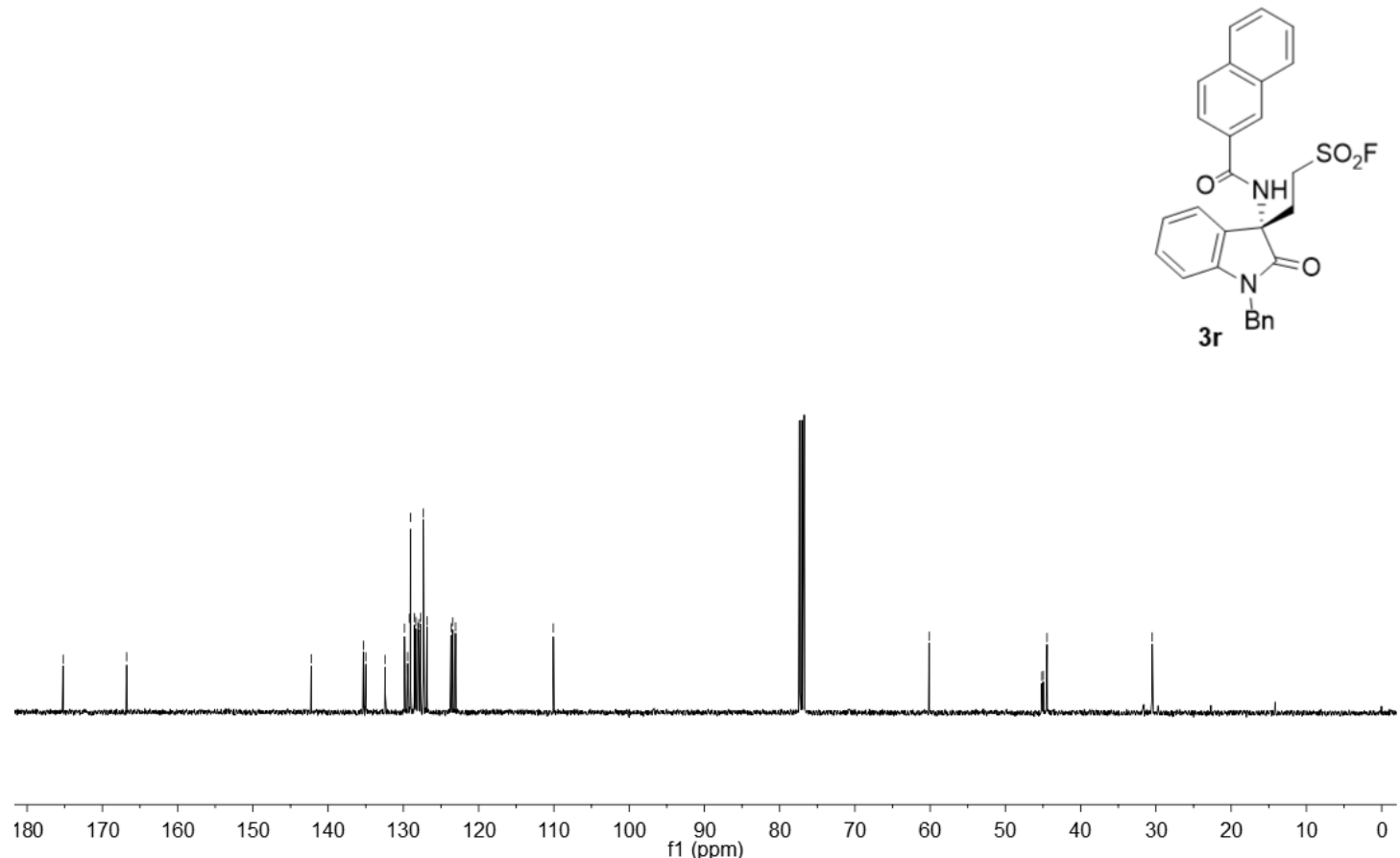

${ }^{1} \mathrm{H},{ }^{19} \mathrm{~F}$ and ${ }^{13} \mathrm{C}$ NMR spectra of $3 \mathbf{r}$

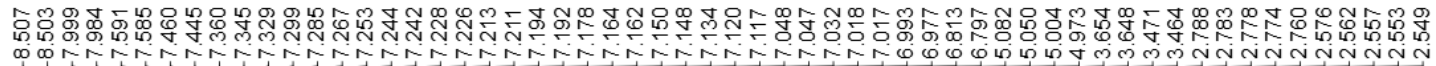

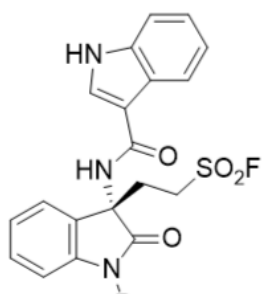

3s

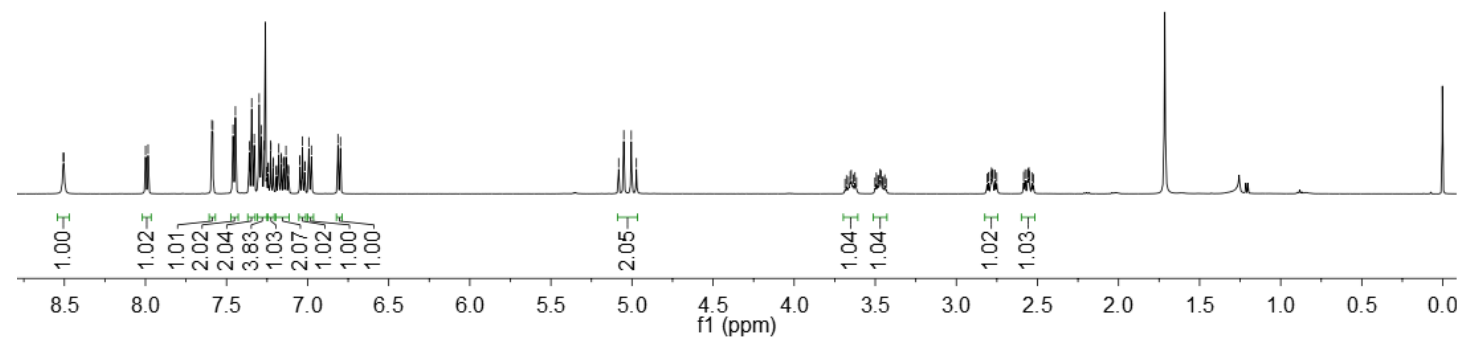




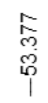

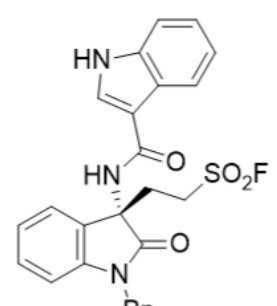

3s $\mathrm{Bn}$

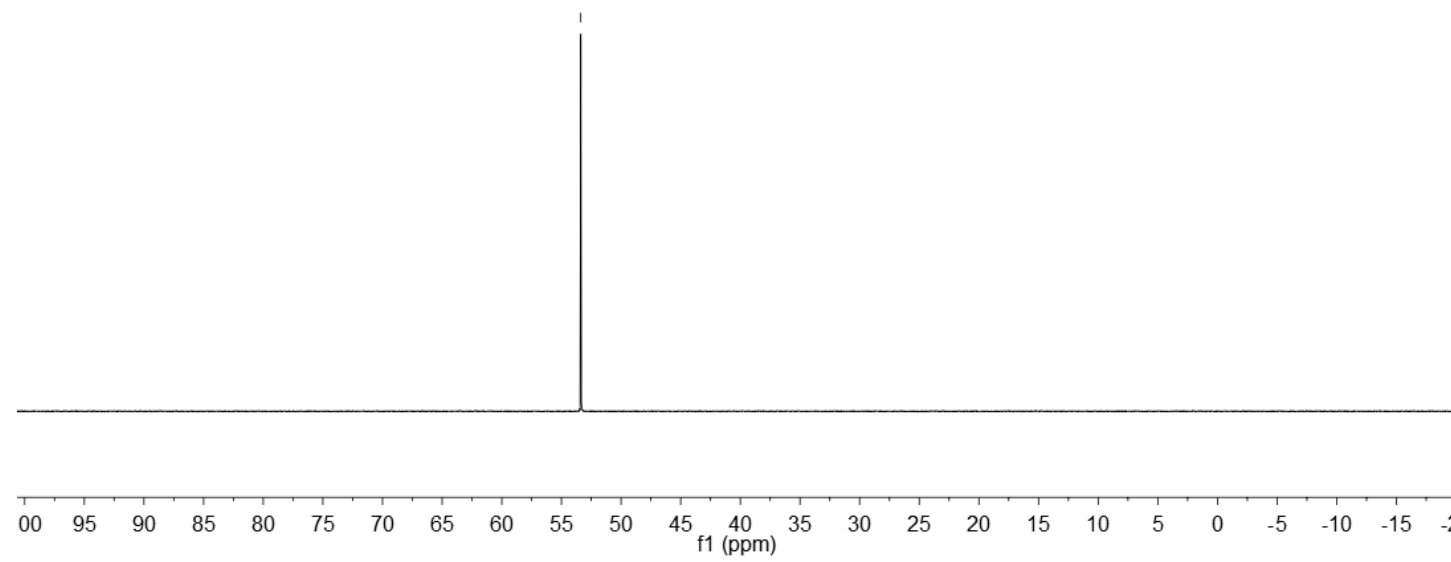

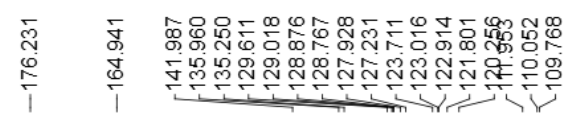

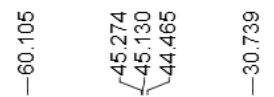

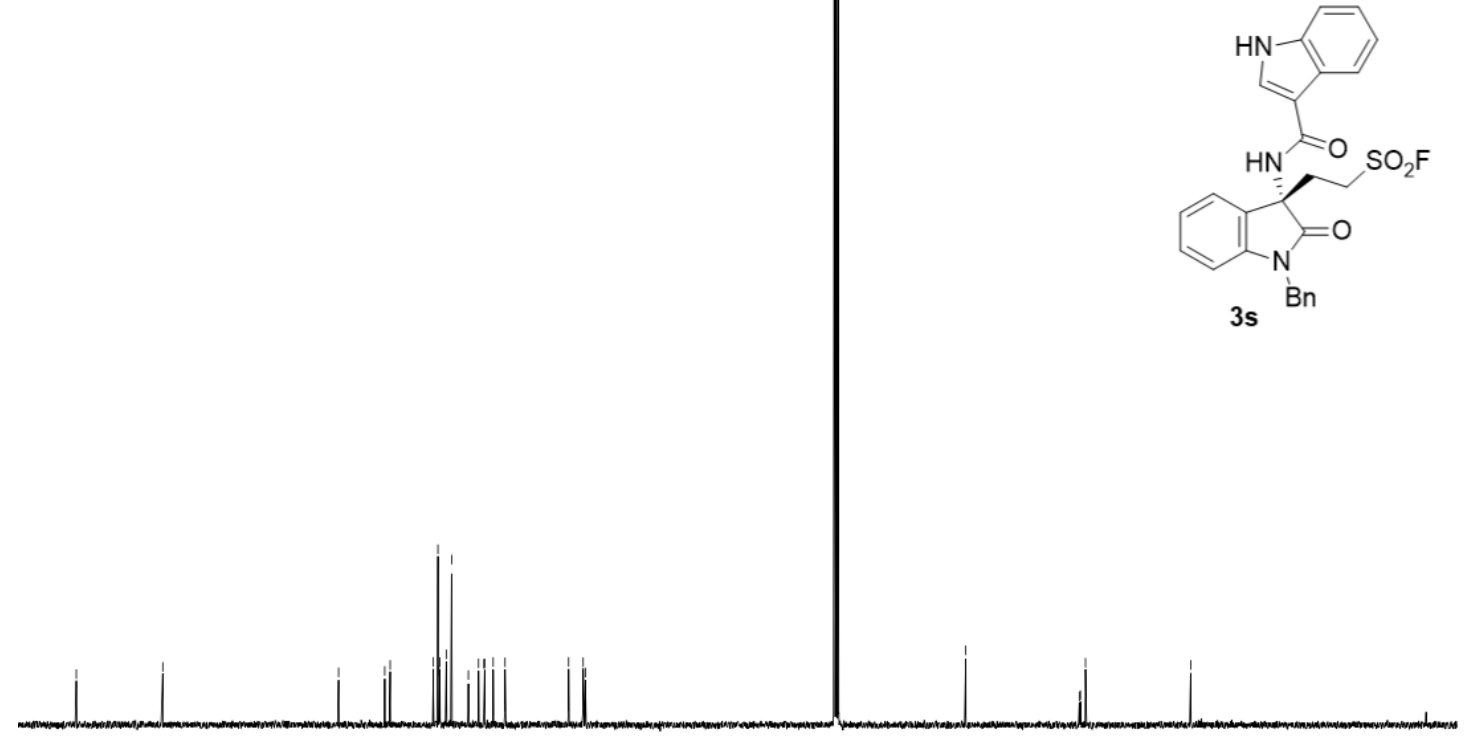

$\begin{array}{lllllllllllllllllll}180 & 170 & 160 & 150 & 140 & 130 & 120 & 110 & 100 & \begin{array}{c}90 \\ \mathrm{f} 1(\mathrm{ppm})\end{array} & 80 & 70 & 60 & 50 & 40 & 30 & 20 & 10 & 0\end{array}$

${ }^{1} \mathrm{H},{ }^{19} \mathrm{~F}$ and ${ }^{13} \mathrm{C}$ NMR spectra of $3 \mathrm{~s}$ 

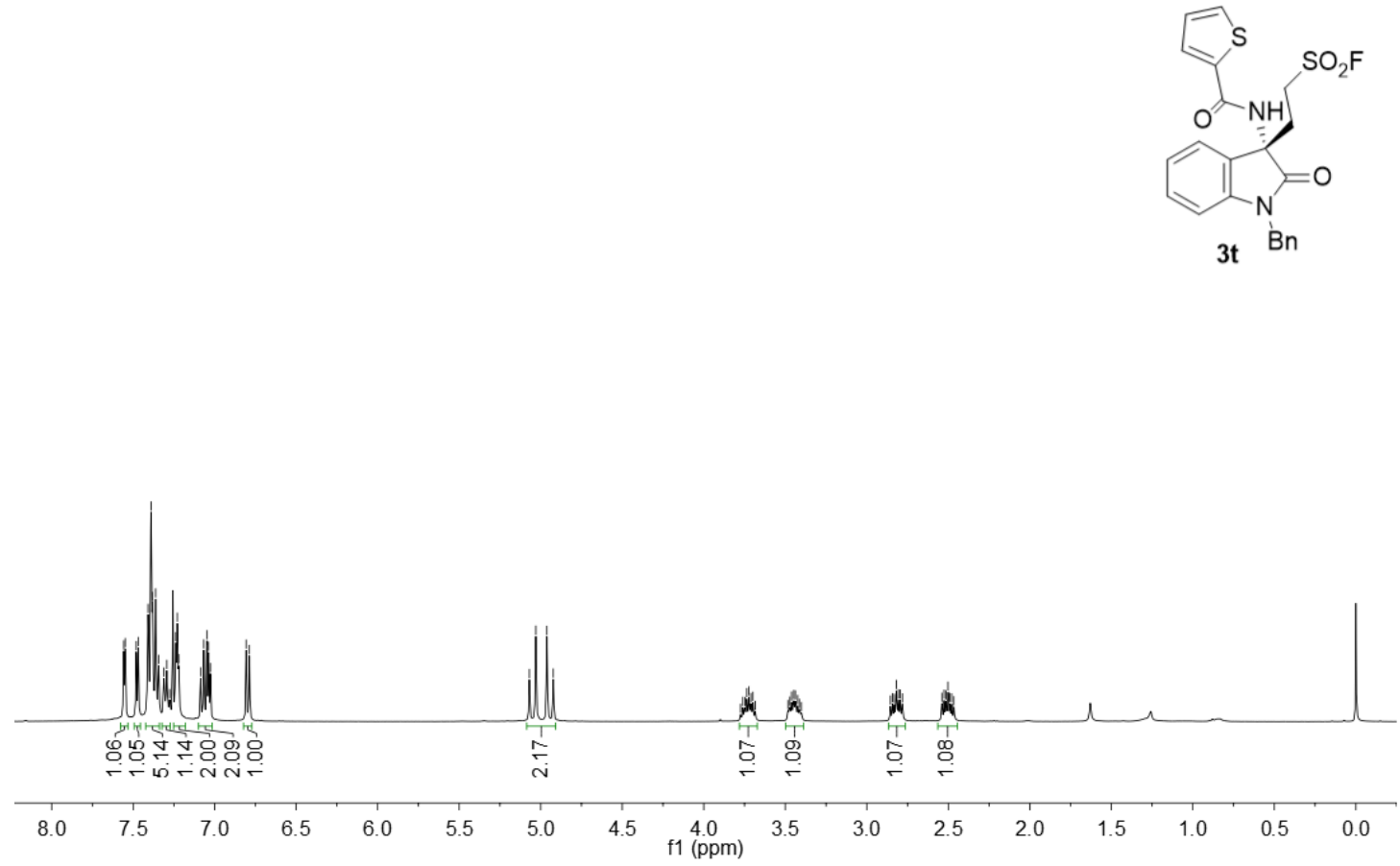

$\underset{\substack{\text { in } \\ \text { in }}}{i}$

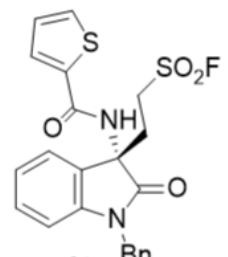

3t Bn

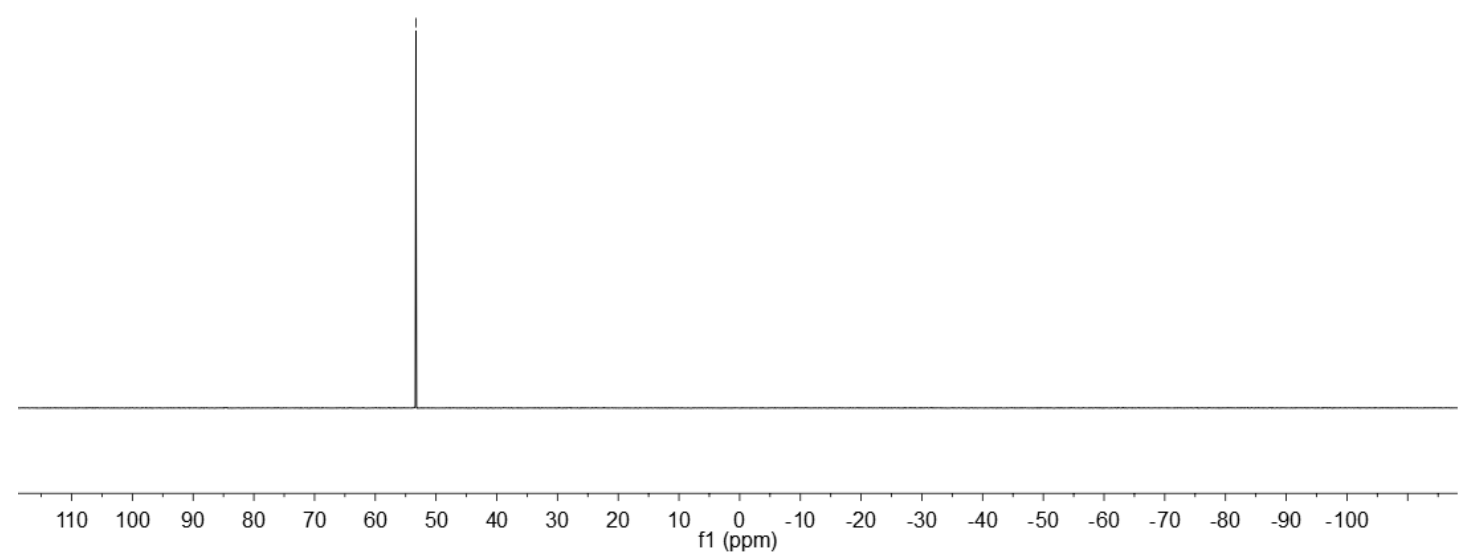



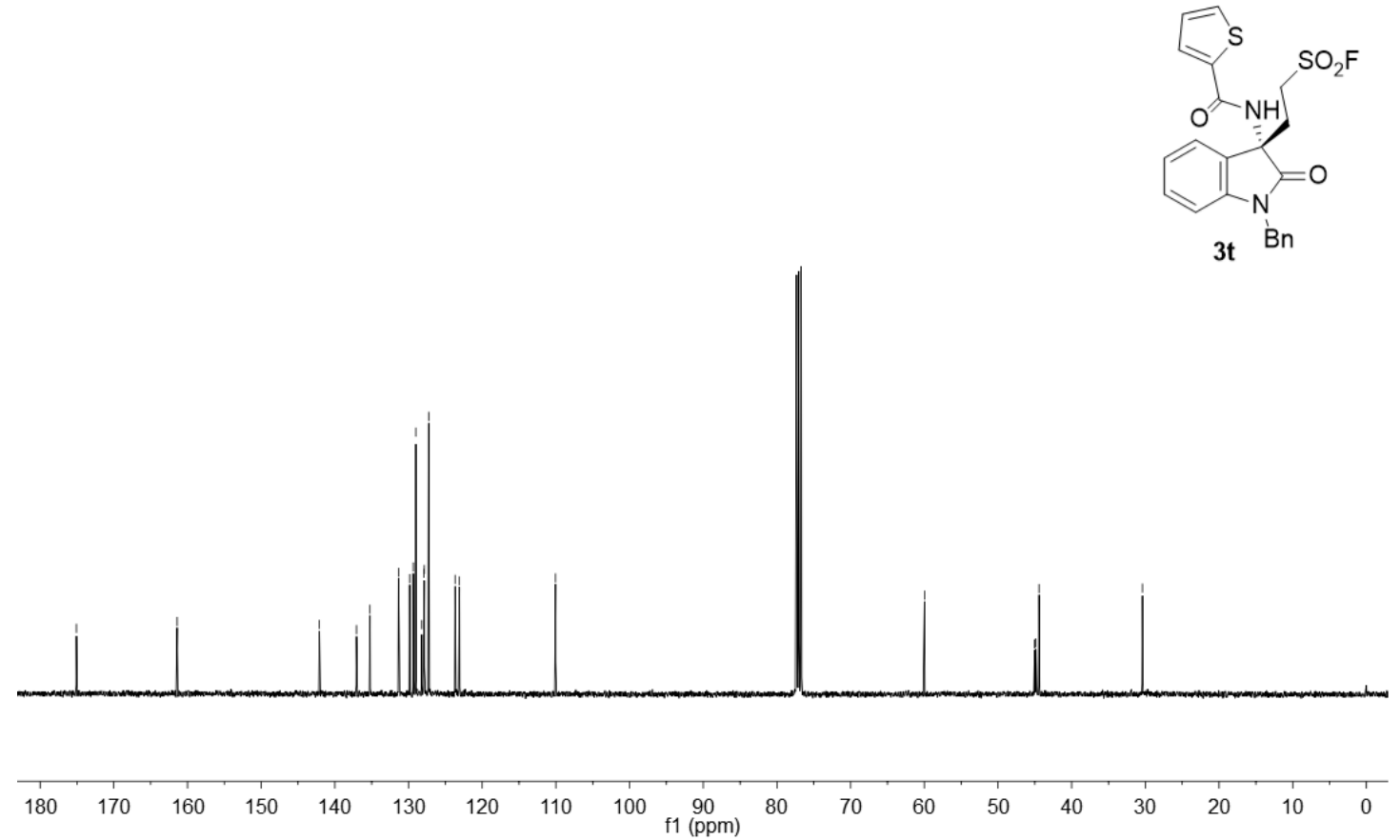

${ }^{1} \mathrm{H},{ }^{19} \mathrm{~F}$ and ${ }^{13} \mathrm{C}$ NMR spectra of $\mathbf{3 t}$

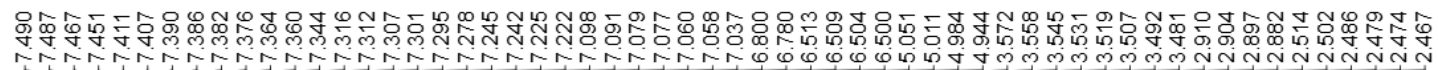
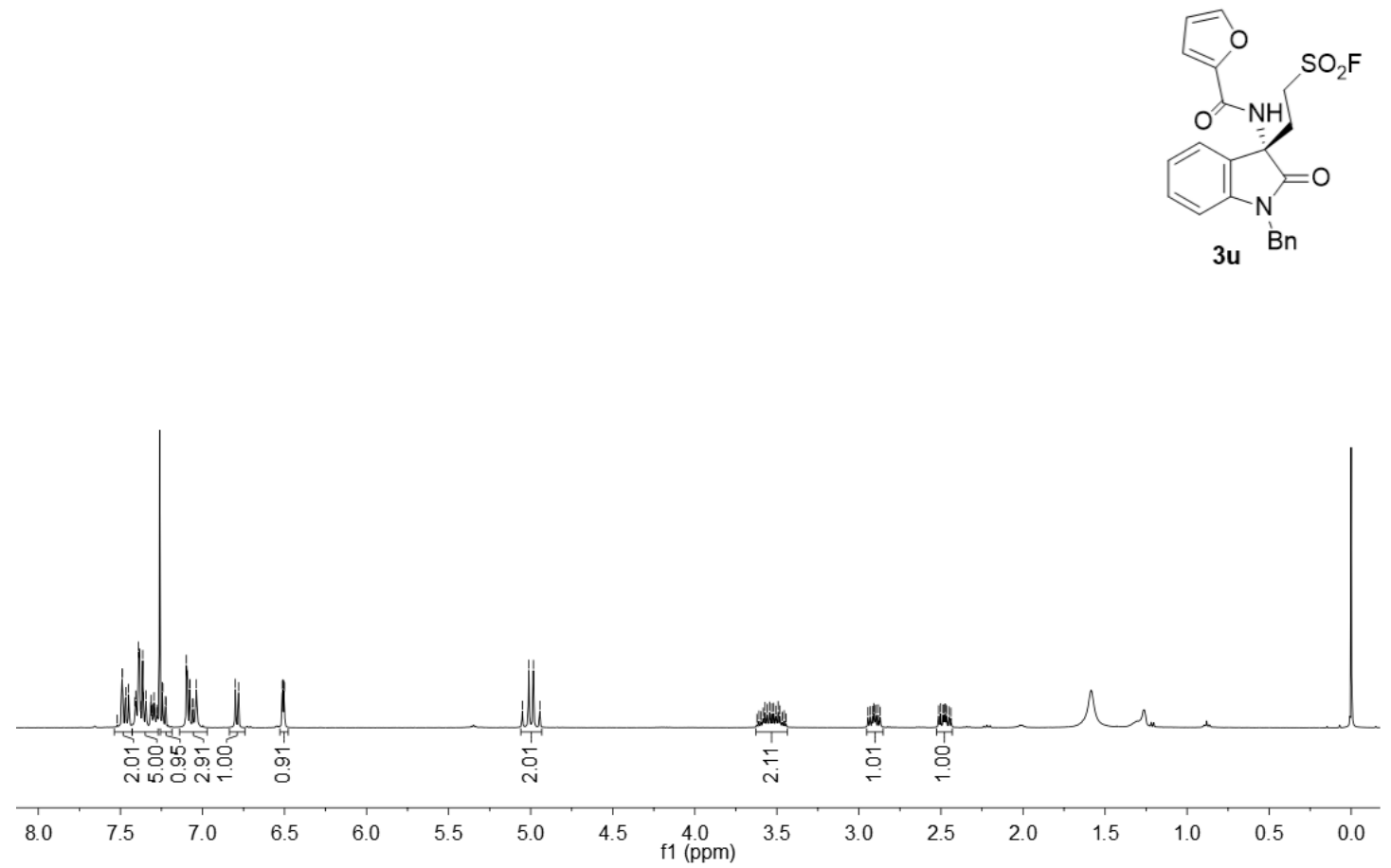

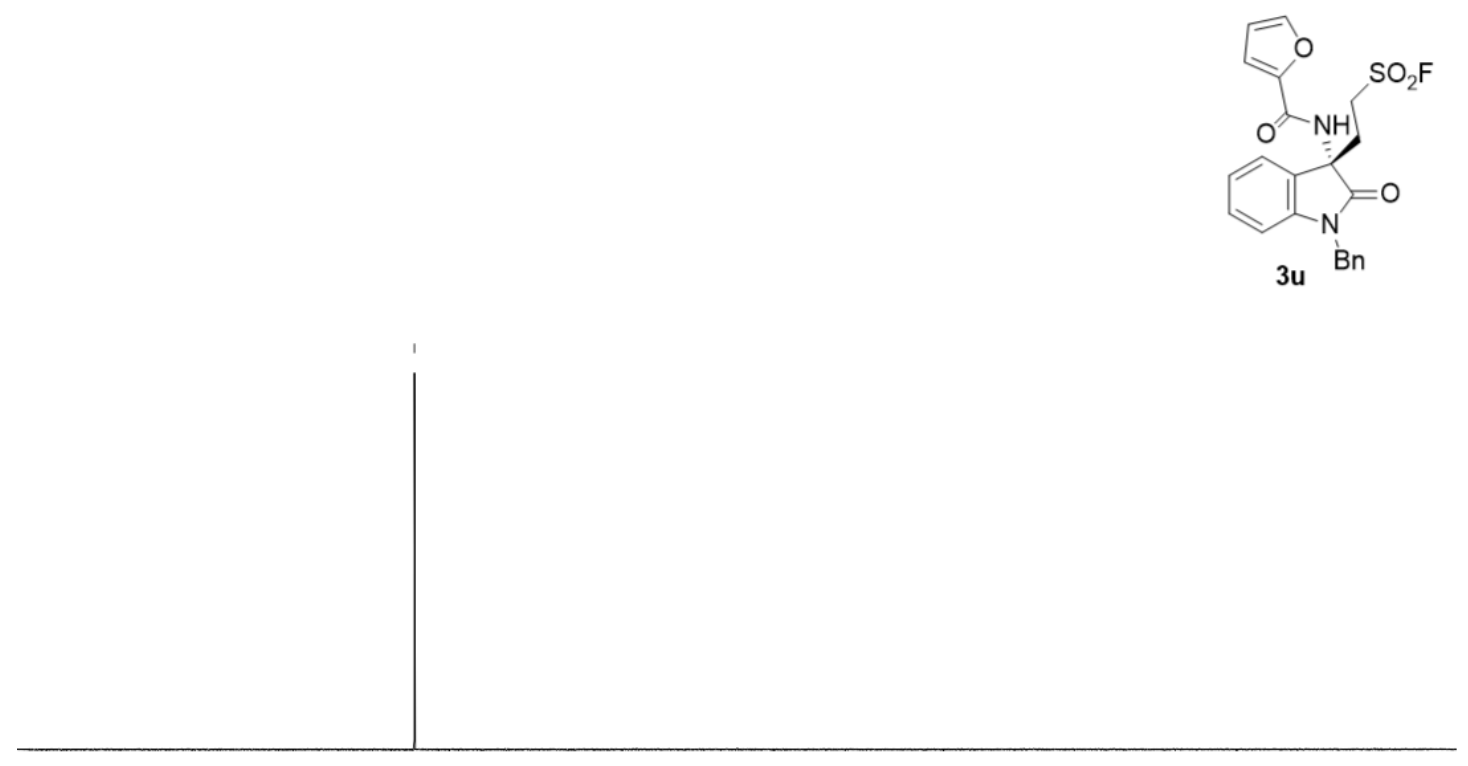

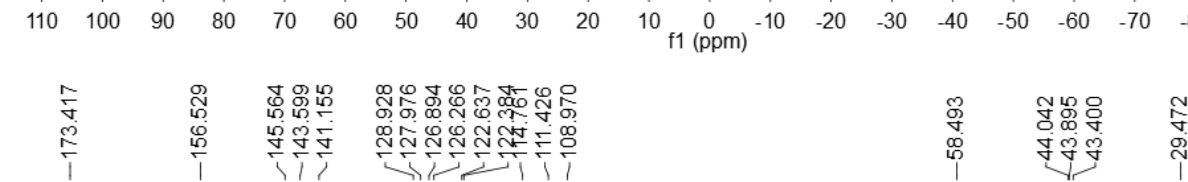

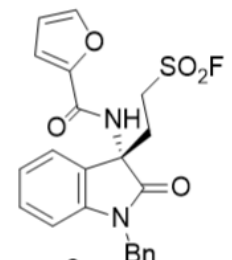

3u Bn

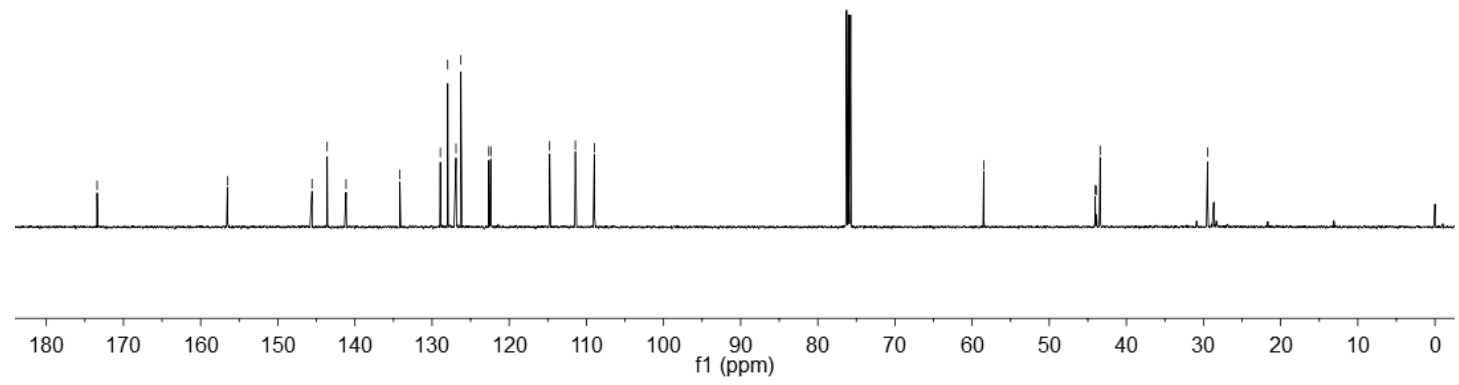

${ }^{1} \mathrm{H},{ }^{19} \mathrm{~F}$ and ${ }^{13} \mathrm{C}$ NMR spectra of $\mathbf{3 u}$ 


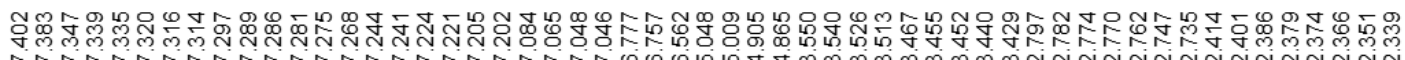

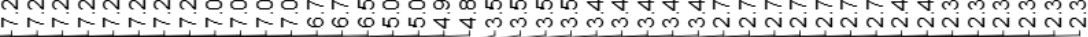

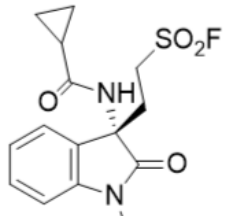

$3 v^{B}$

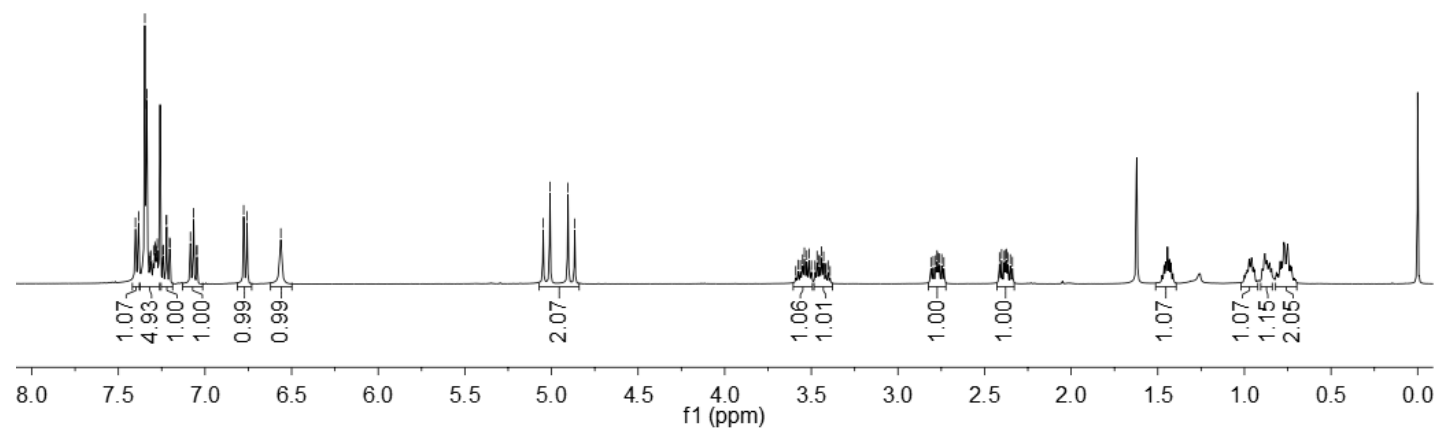

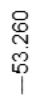

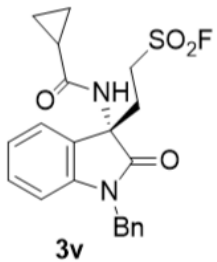

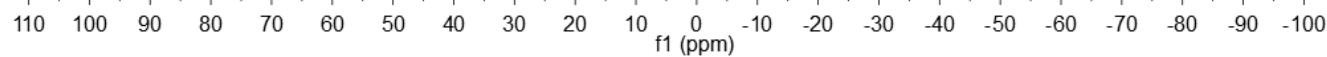



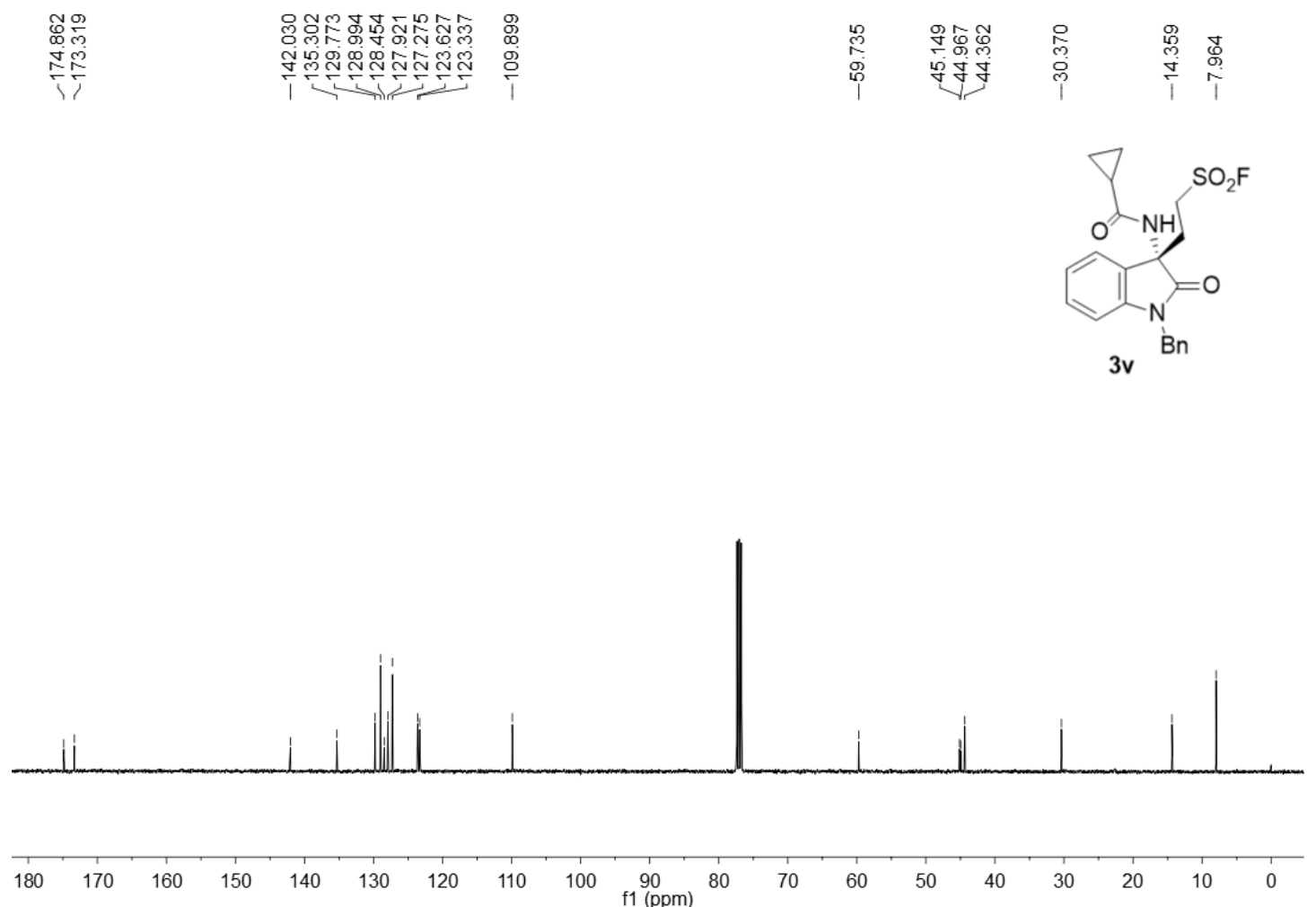

${ }^{1} \mathrm{H},{ }^{19} \mathrm{~F}$ and ${ }^{13} \mathrm{C}$ NMR spectra of $3 \mathbf{v}$

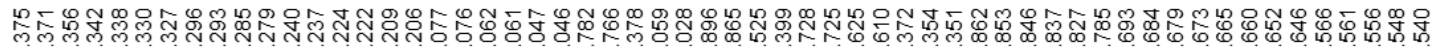

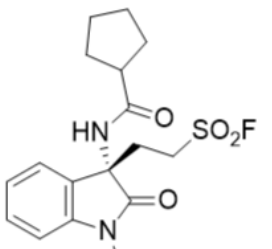

3w Bn

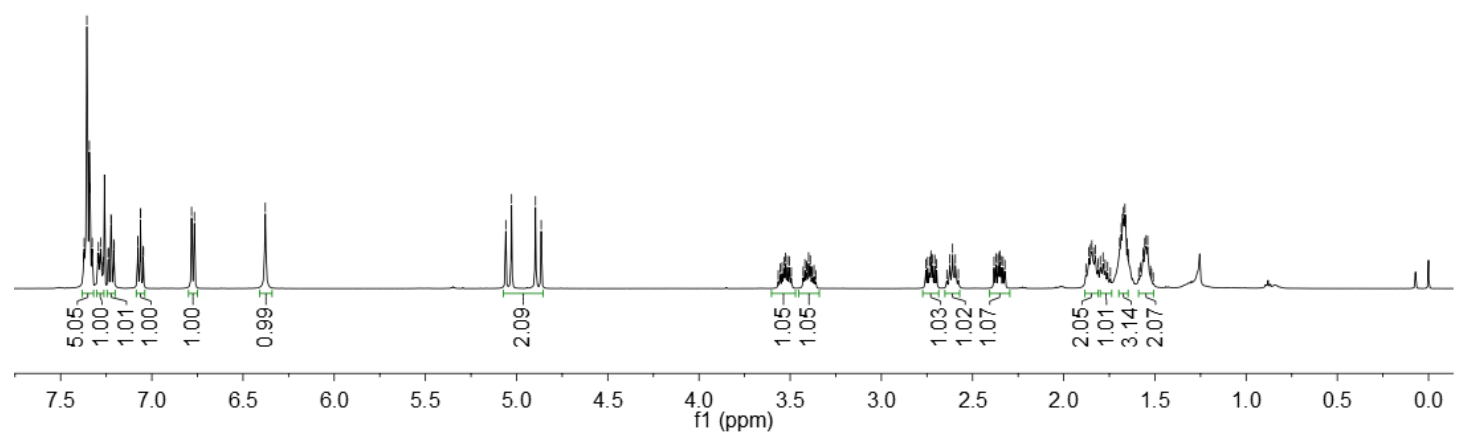




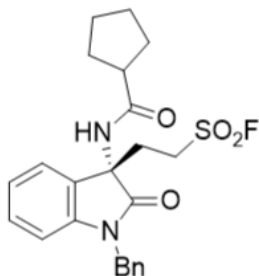

$3 w$

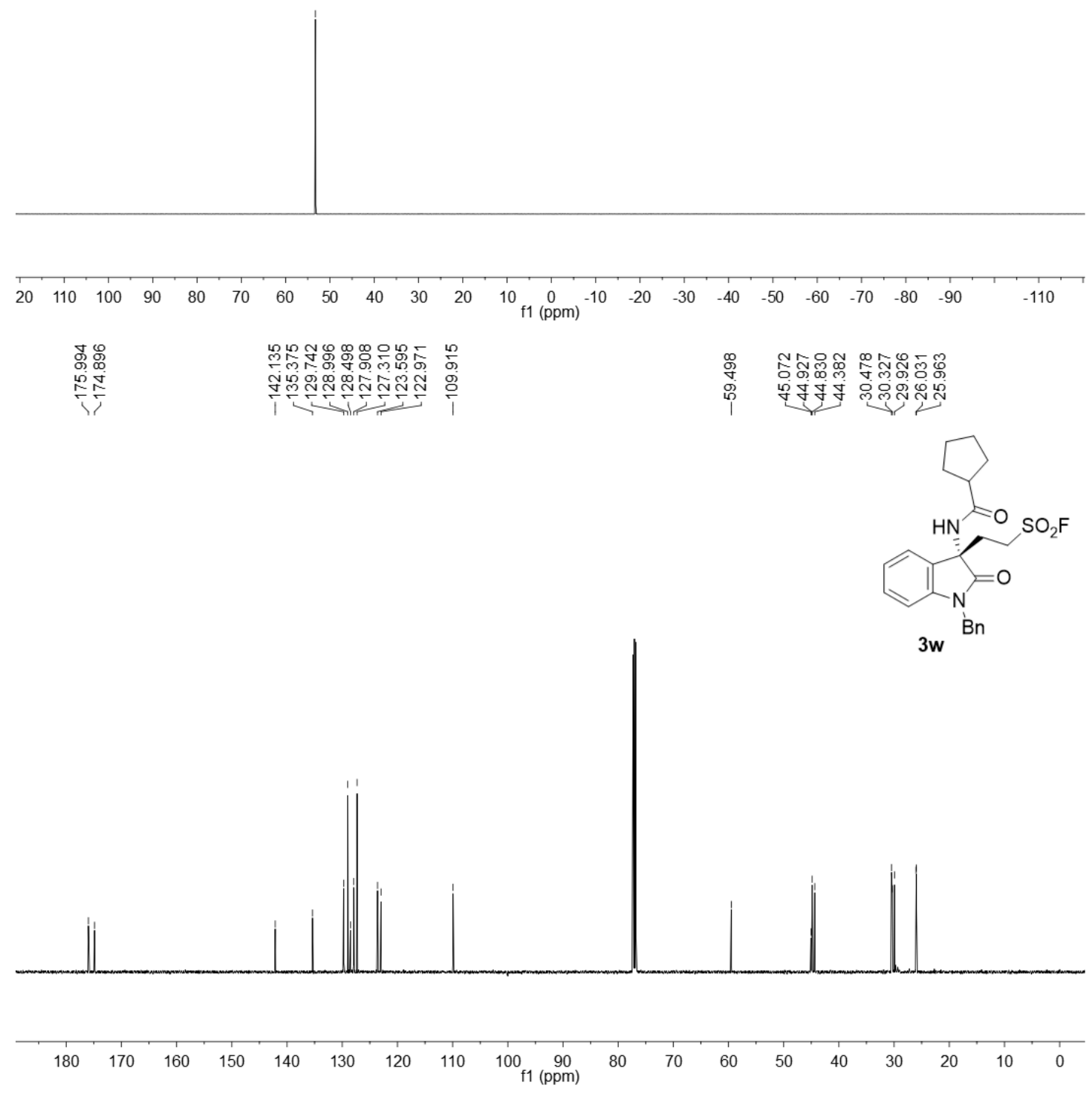

${ }^{1} \mathrm{H},{ }^{19} \mathrm{~F}$ and ${ }^{13} \mathrm{C}$ NMR spectra of $3 \mathbf{w}$ 


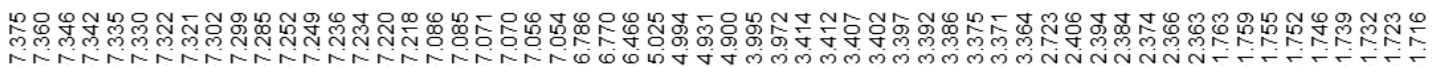
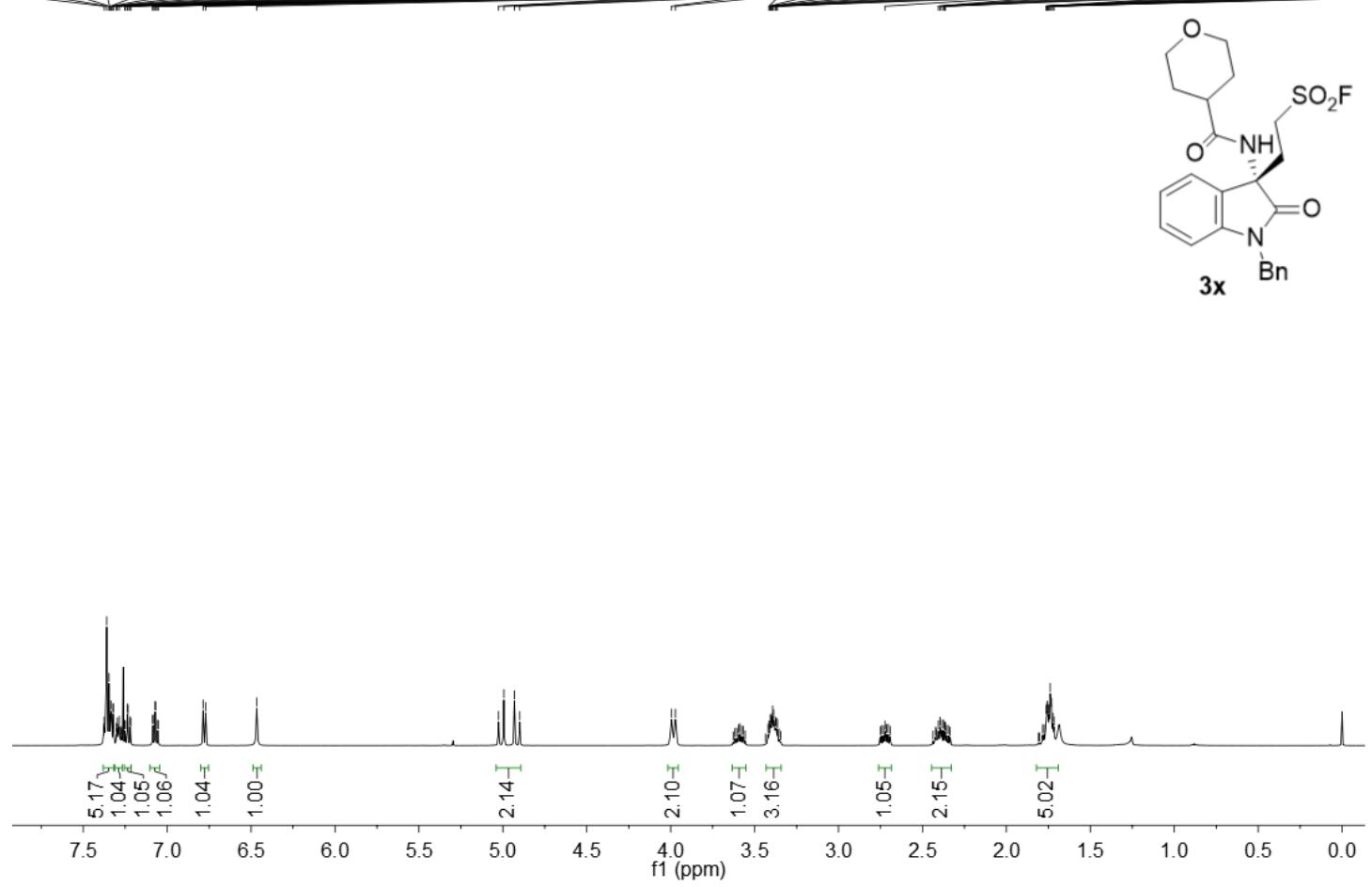

ֻ

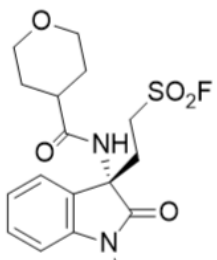

$3 \times \quad B n$

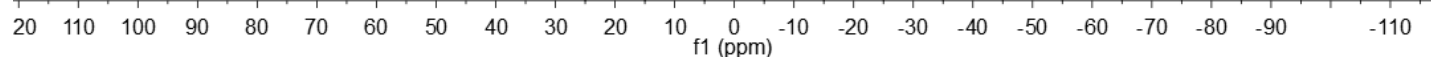




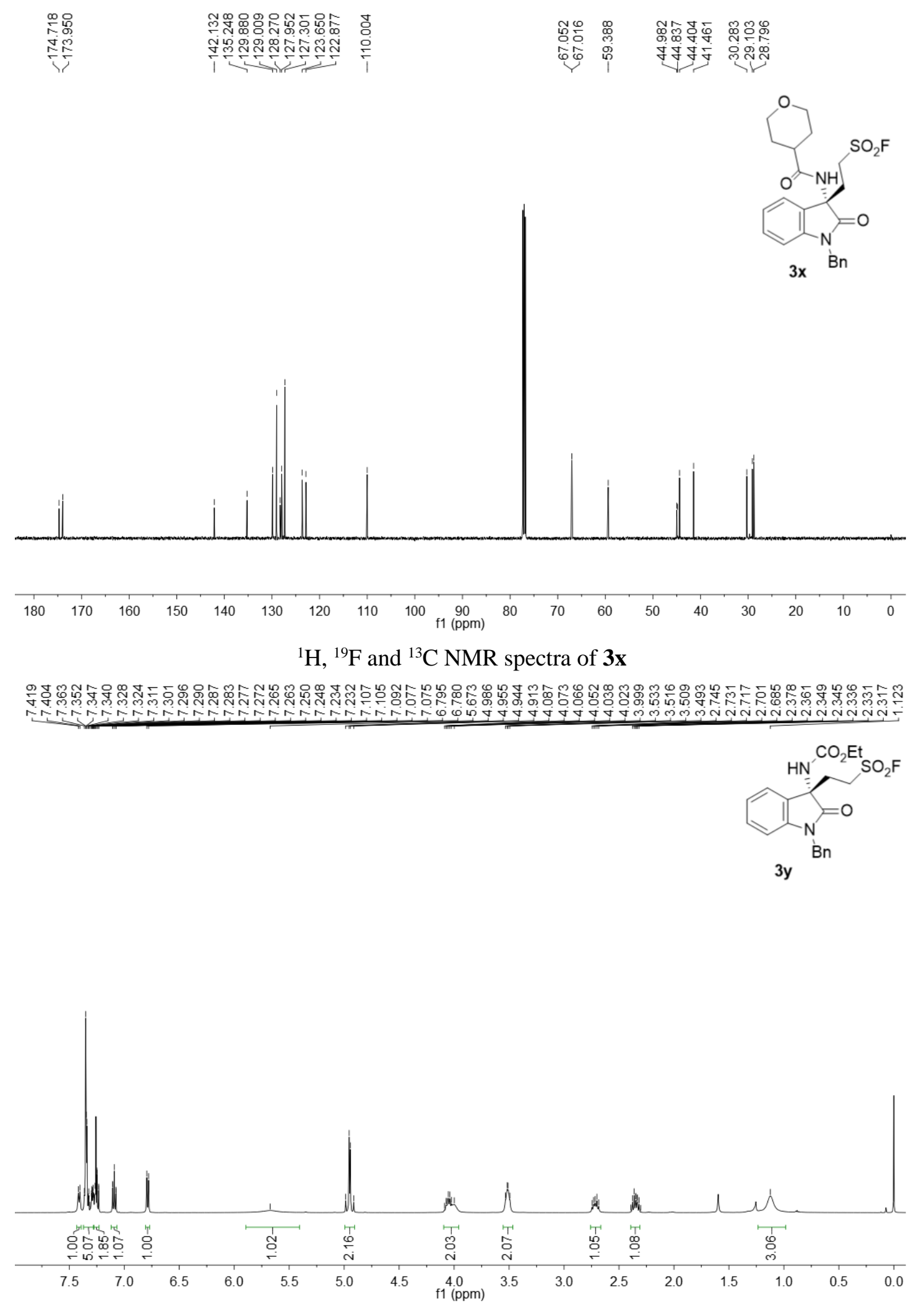




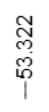
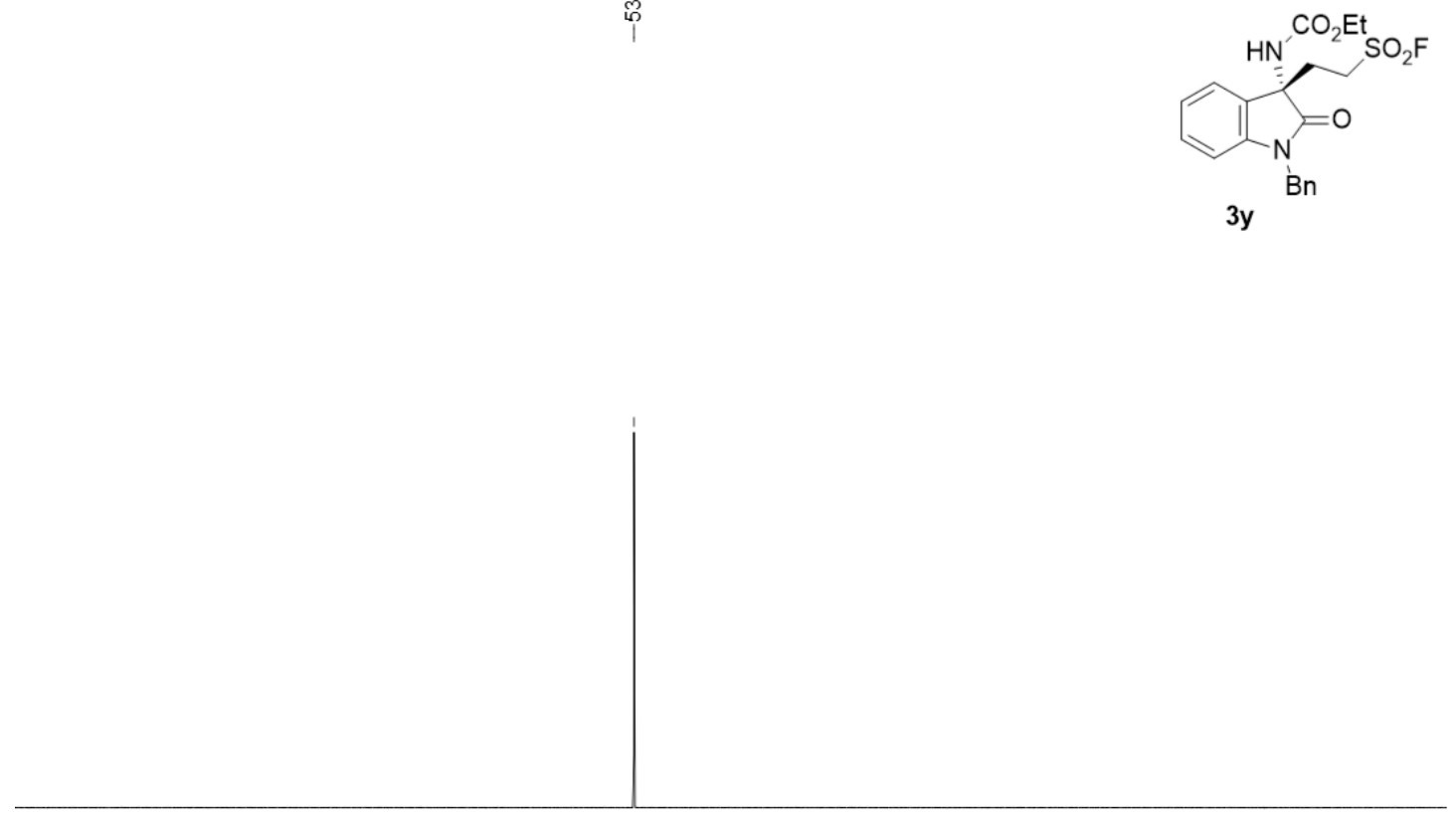

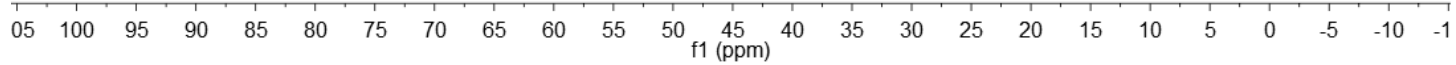

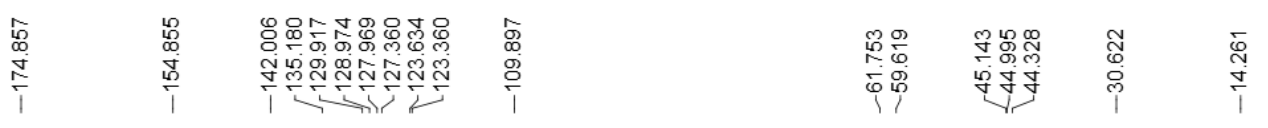

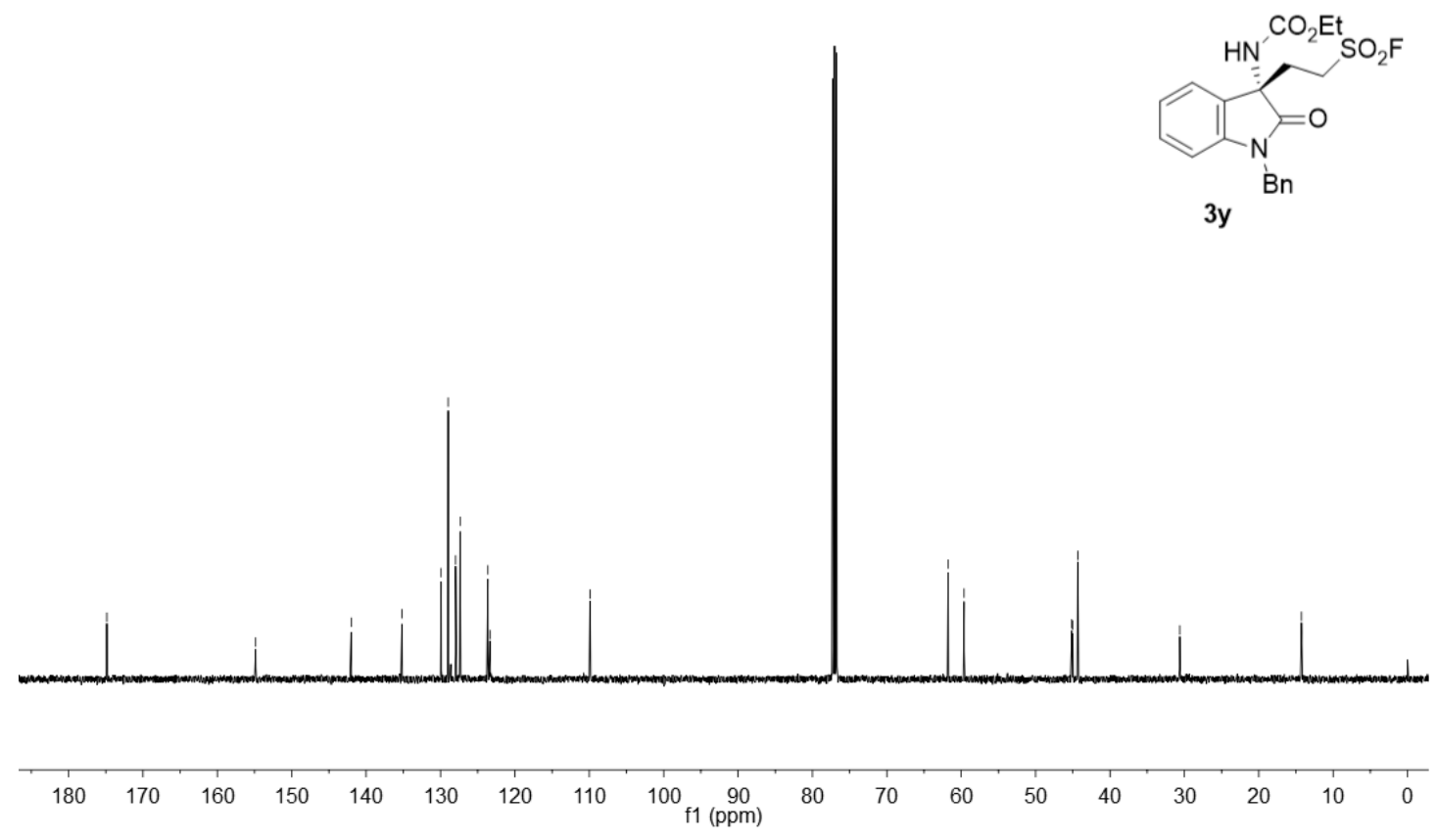

${ }^{1} \mathrm{H},{ }^{19} \mathrm{~F}$ and ${ }^{13} \mathrm{C}$ NMR spectra of $3 \mathbf{y}$ 


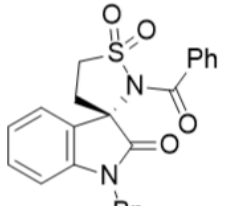

$5 a^{\mathrm{Bn}}$
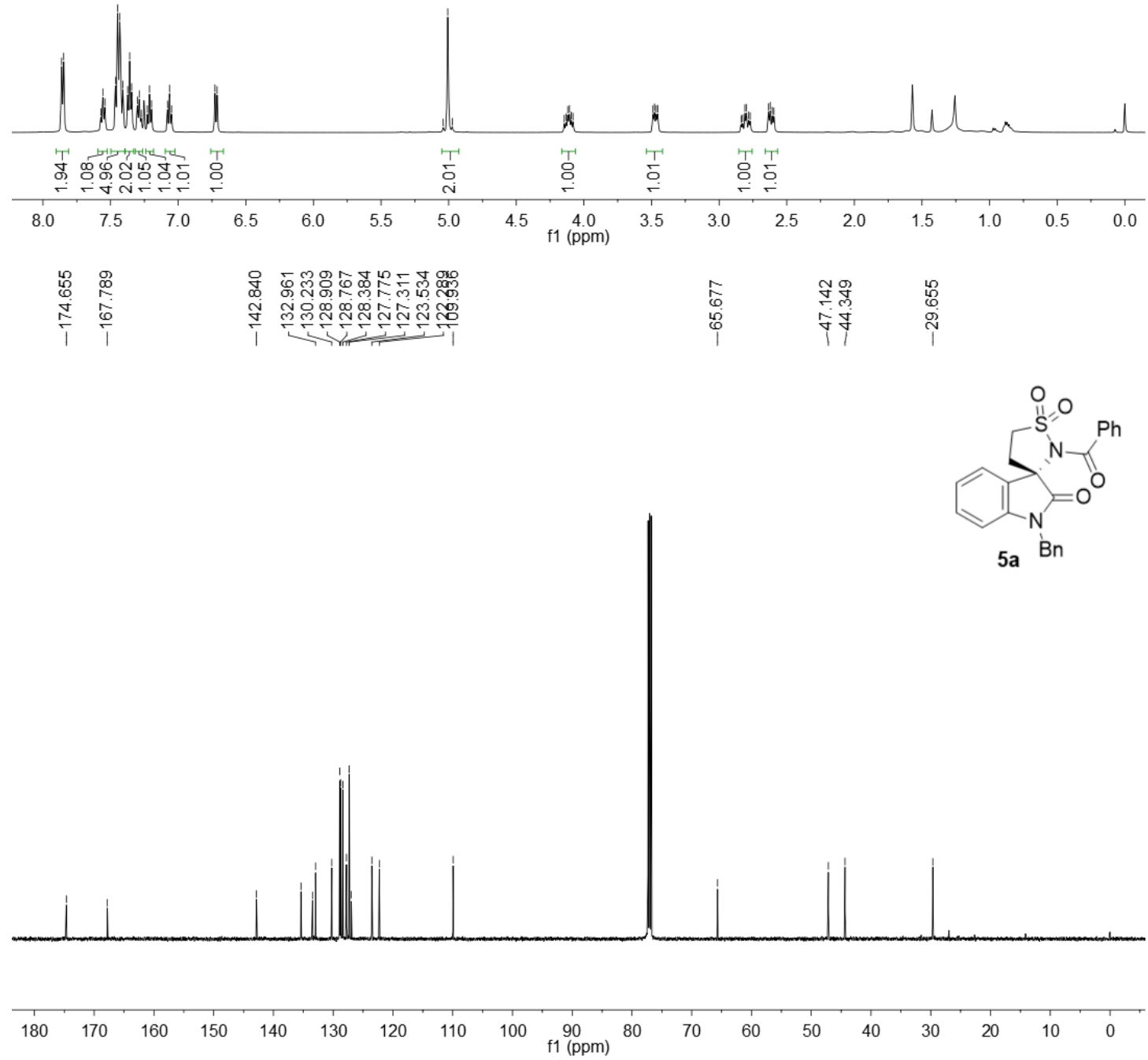

${ }^{1} \mathrm{H}$ and ${ }^{13} \mathrm{C}$ NMR spectra of $\mathbf{5 a}$ 

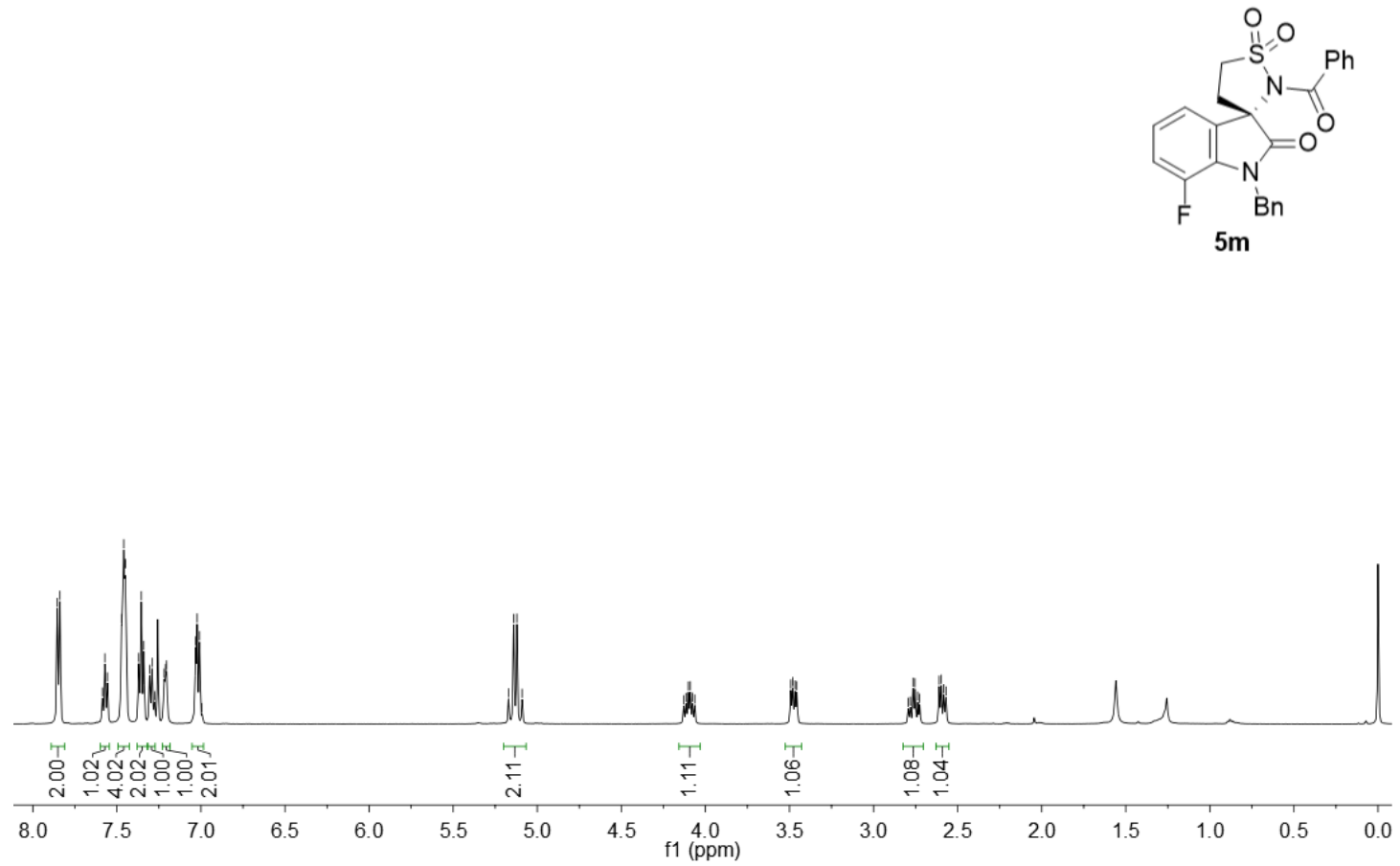

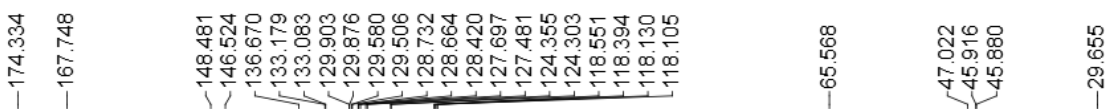

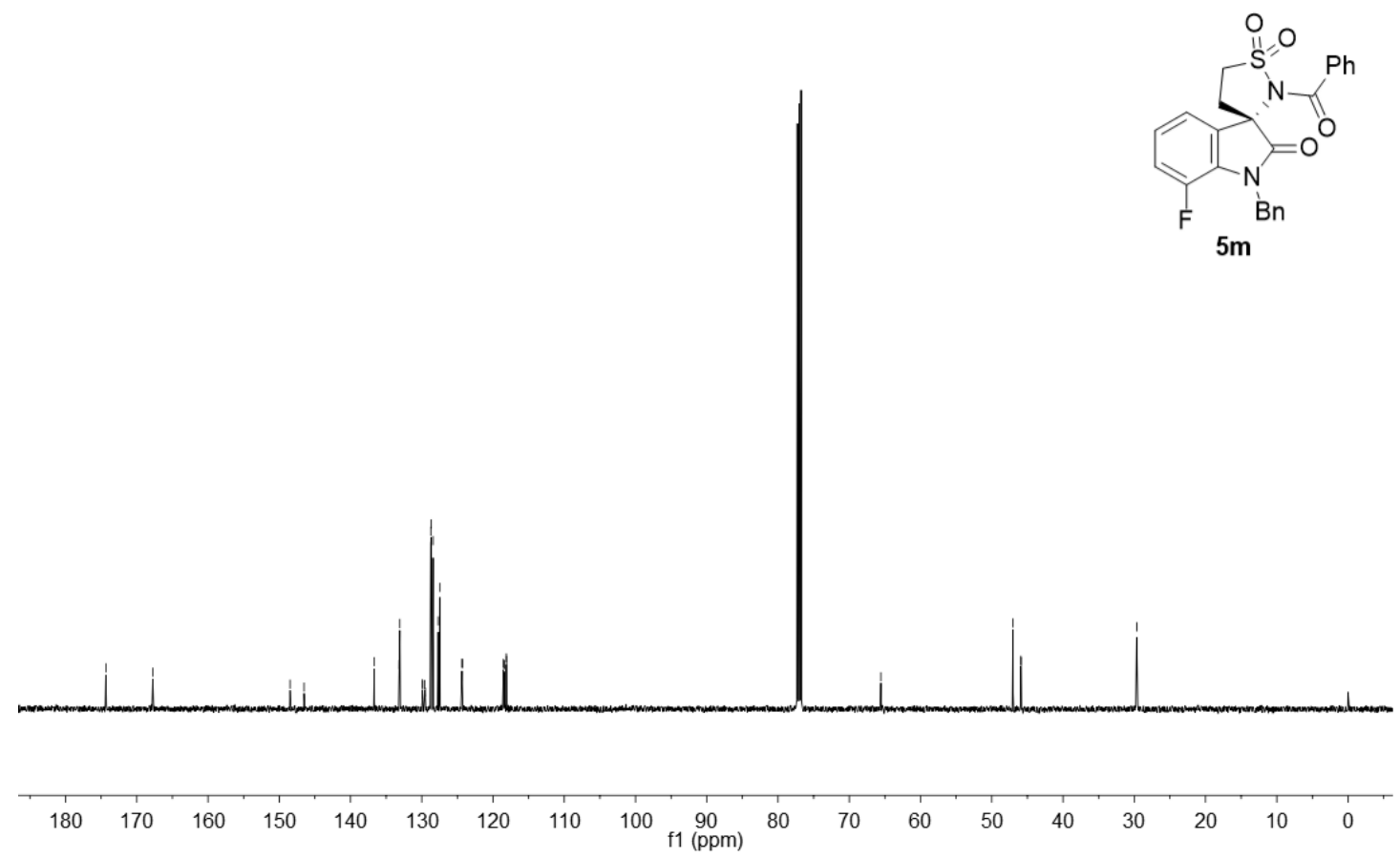

${ }^{1} \mathrm{H}$ and ${ }^{13} \mathrm{C}$ NMR spectra of $5 \mathrm{~m}$ 

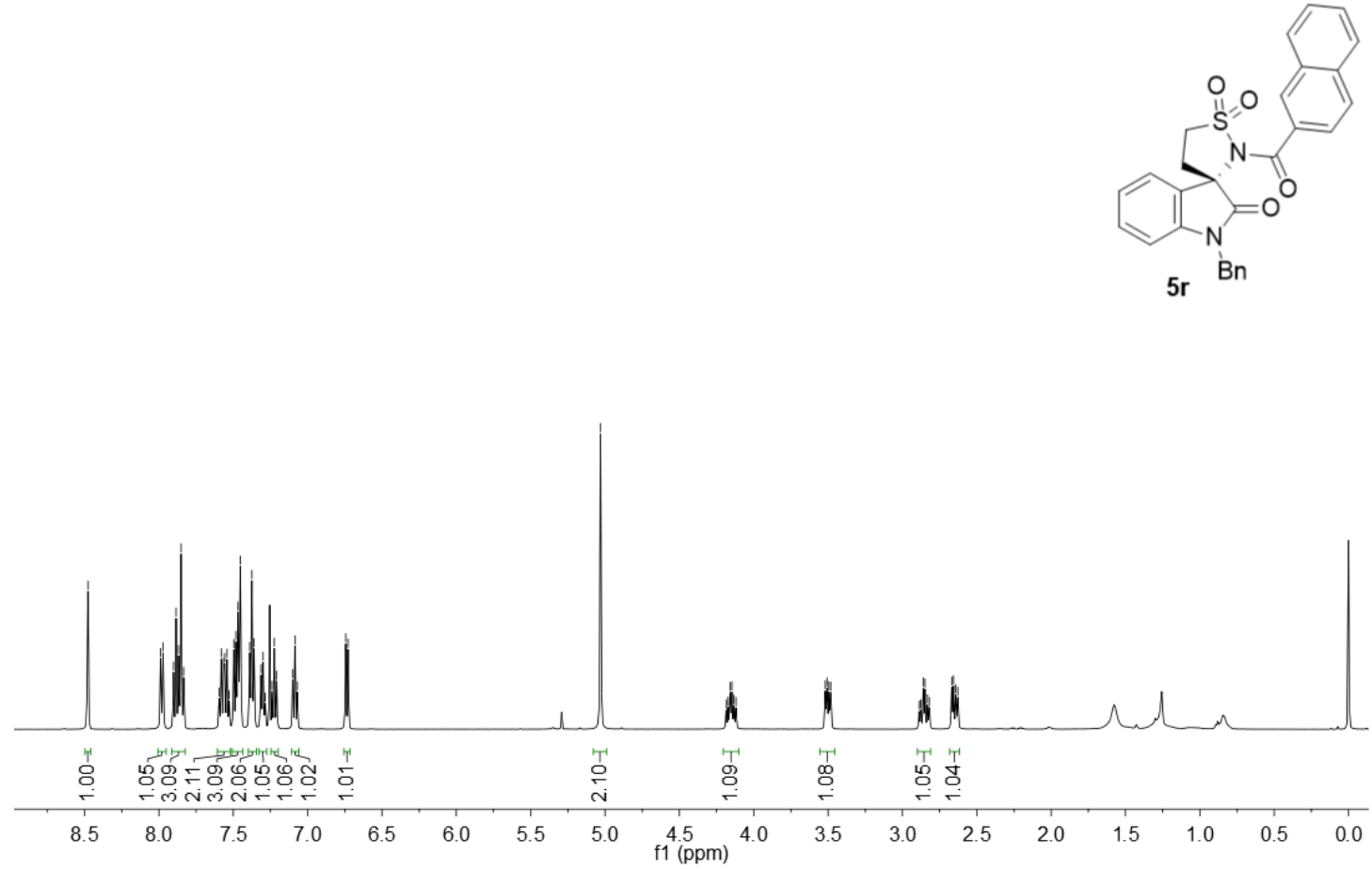

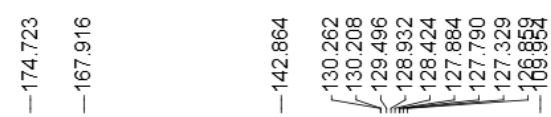

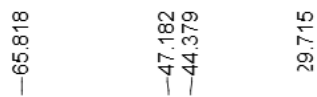

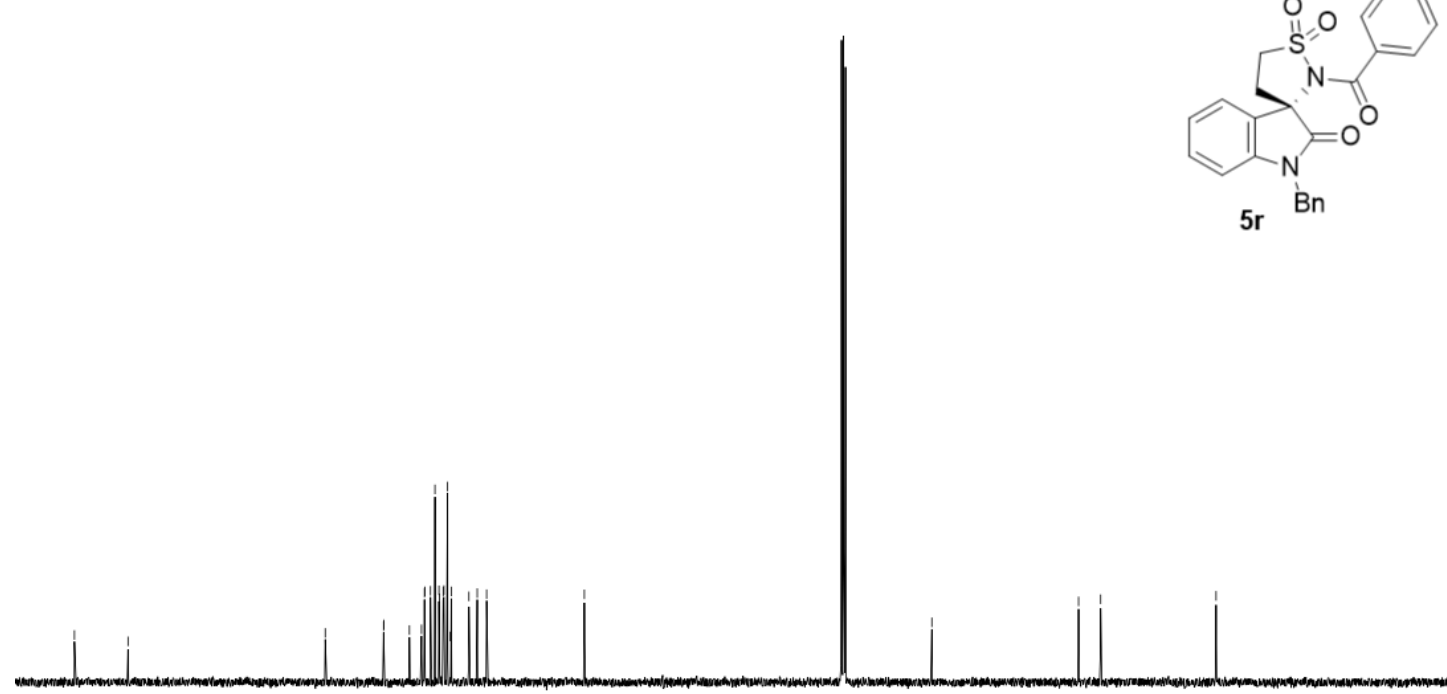

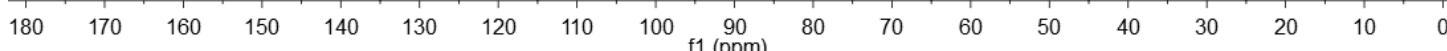

${ }^{1} \mathrm{H}$ and ${ }^{13} \mathrm{C}$ NMR spectra of $5 \mathbf{r}$ 

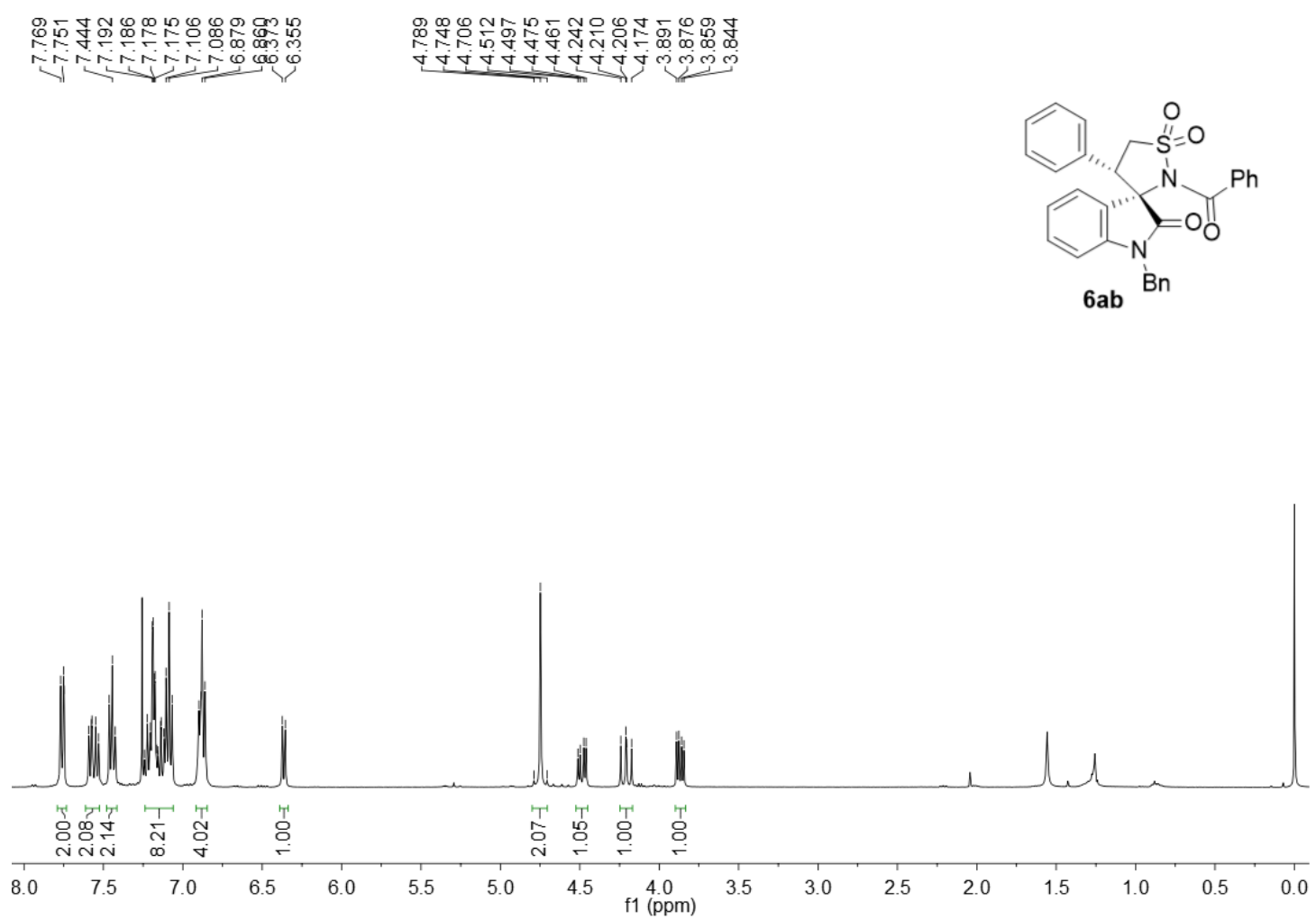

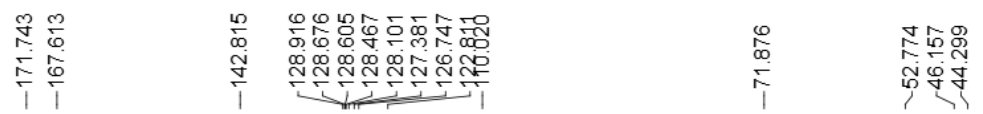

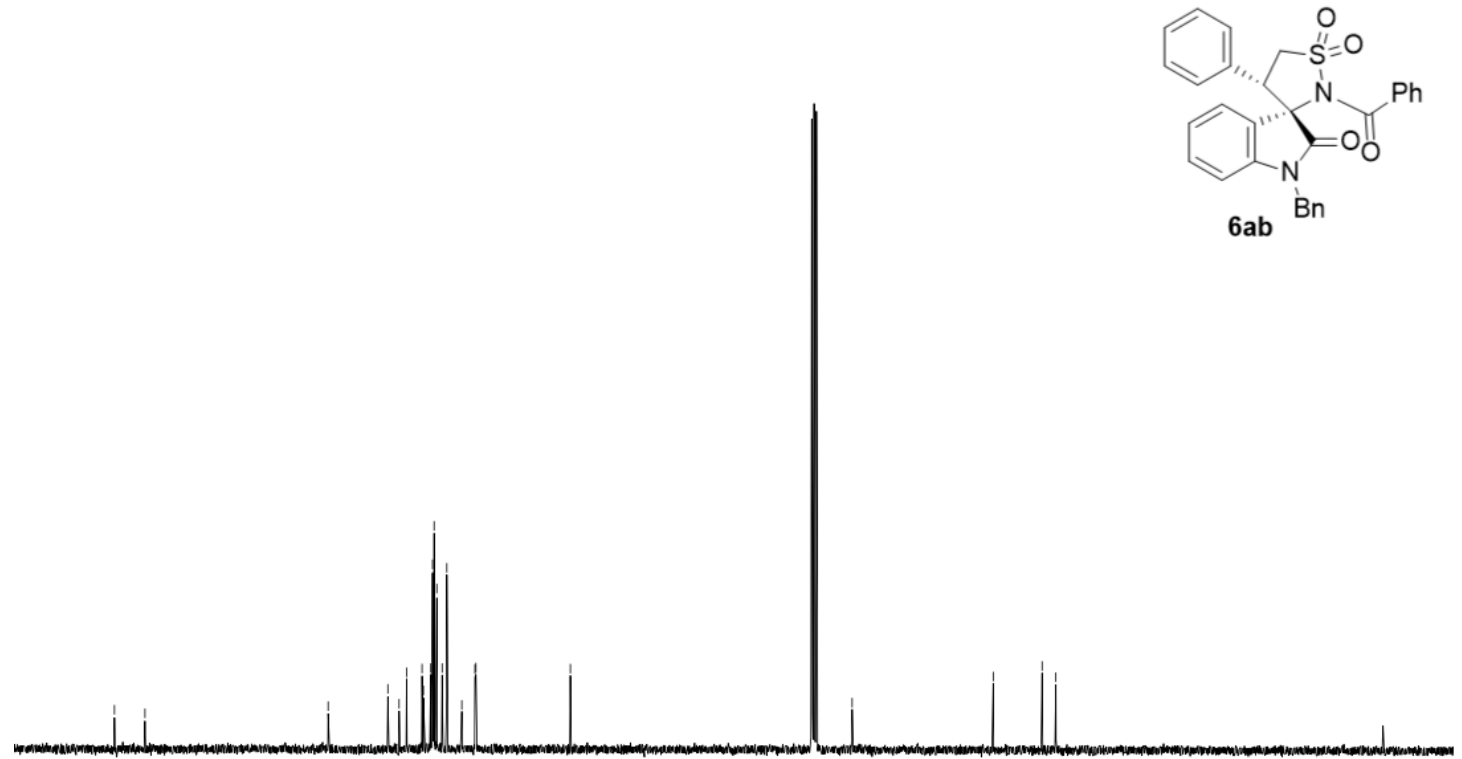

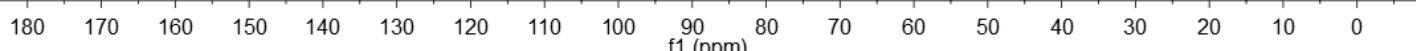

${ }^{1} \mathrm{H}$ and ${ }^{13} \mathrm{C}$ NMR spectra of $\mathbf{6 a b}$ 
$\mathrm{Cl}$

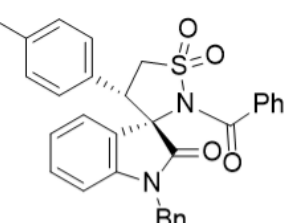

$6 a^{B n}$

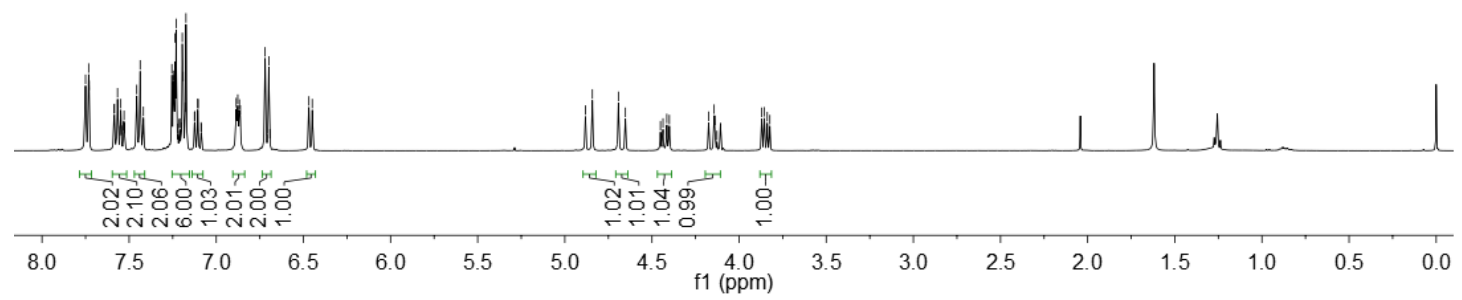

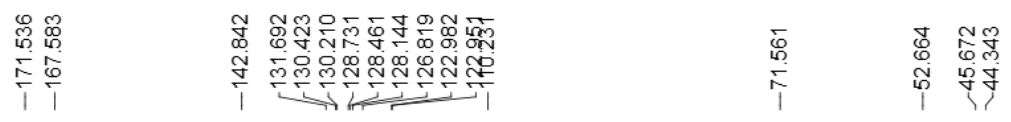
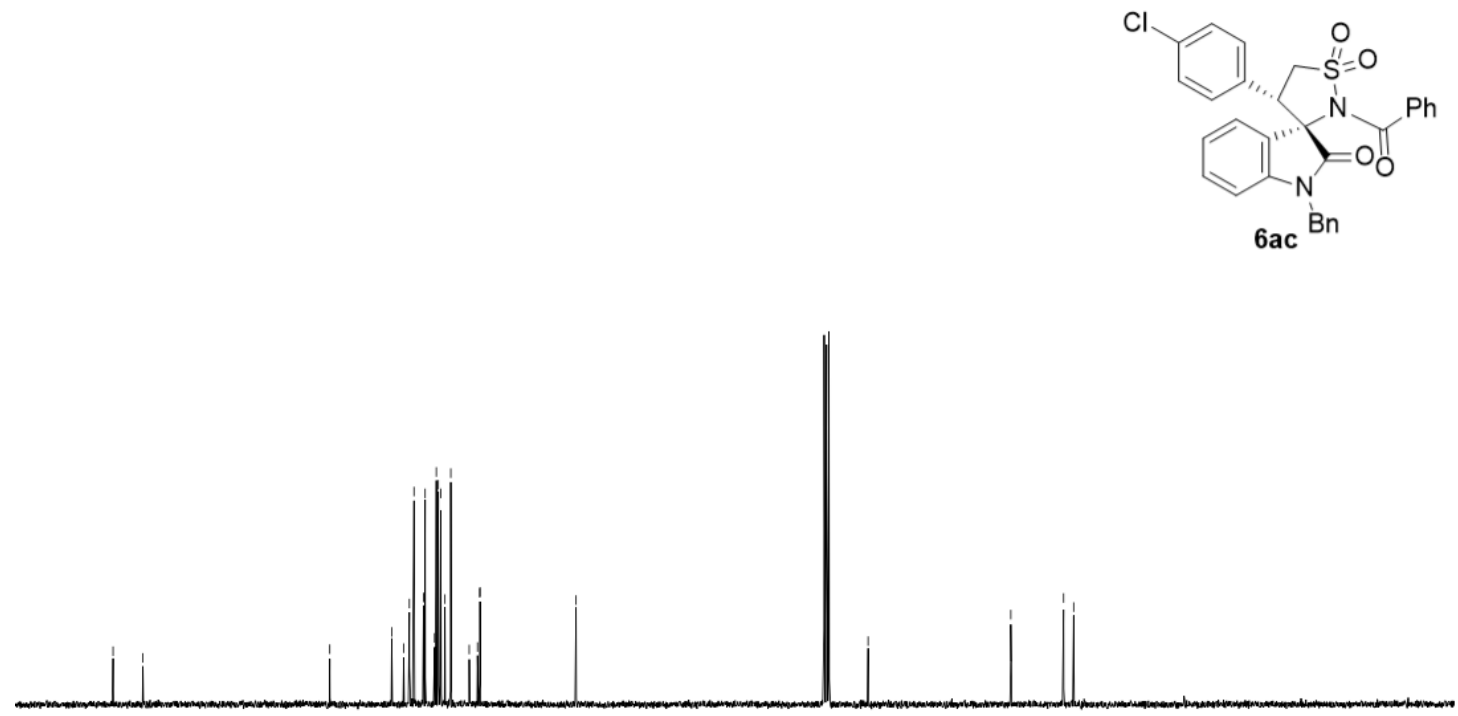

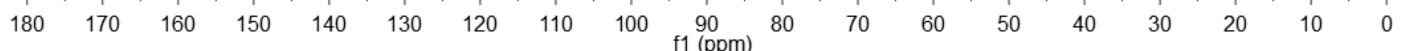

${ }^{1} \mathrm{H}$ and ${ }^{13} \mathrm{C}$ NMR spectra of $\mathbf{6 a c}$ 

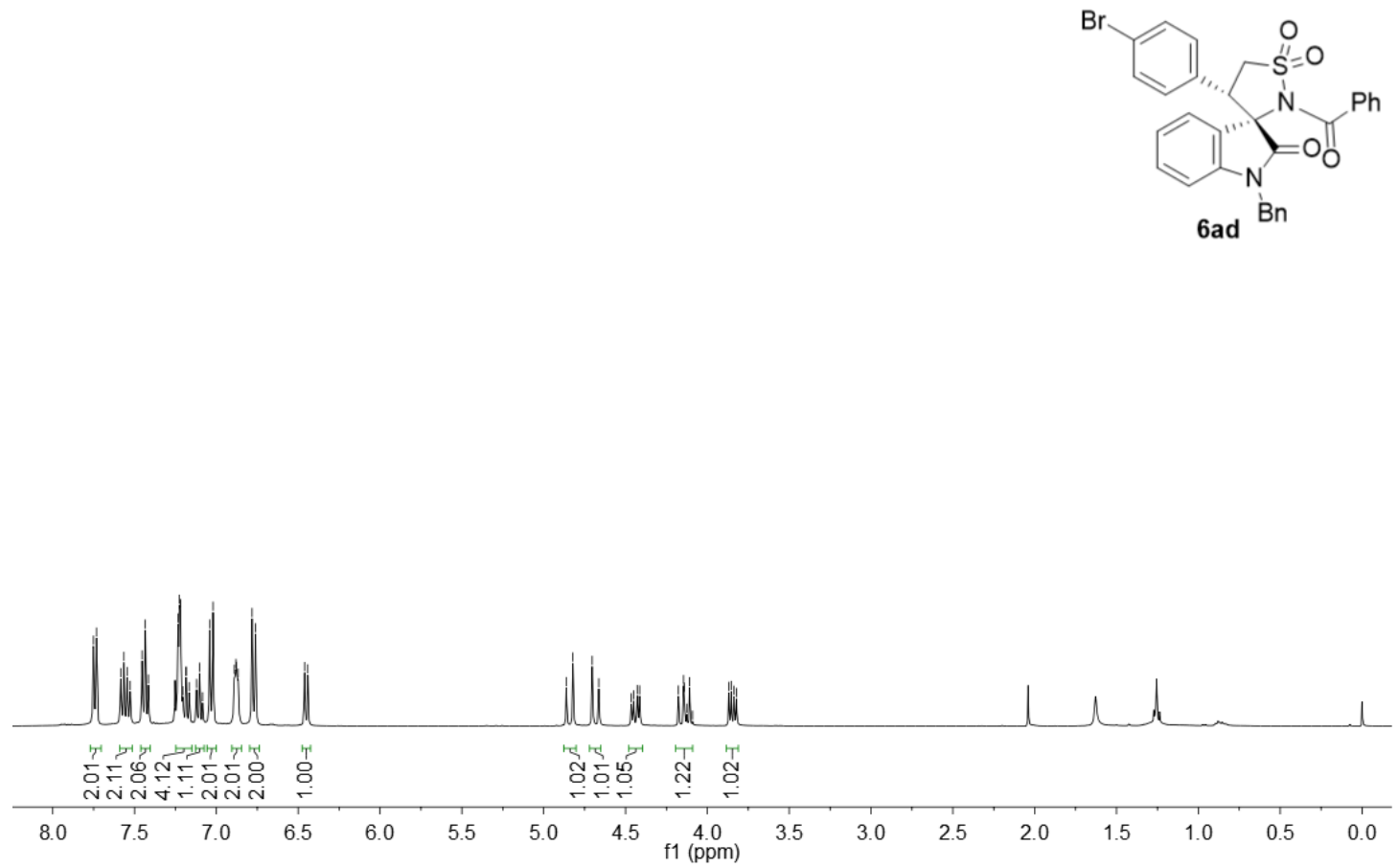

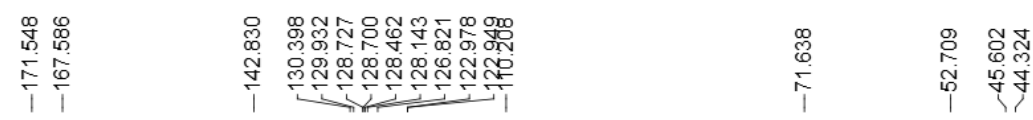

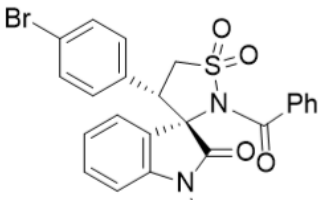

6ad $\mathrm{Bn}$

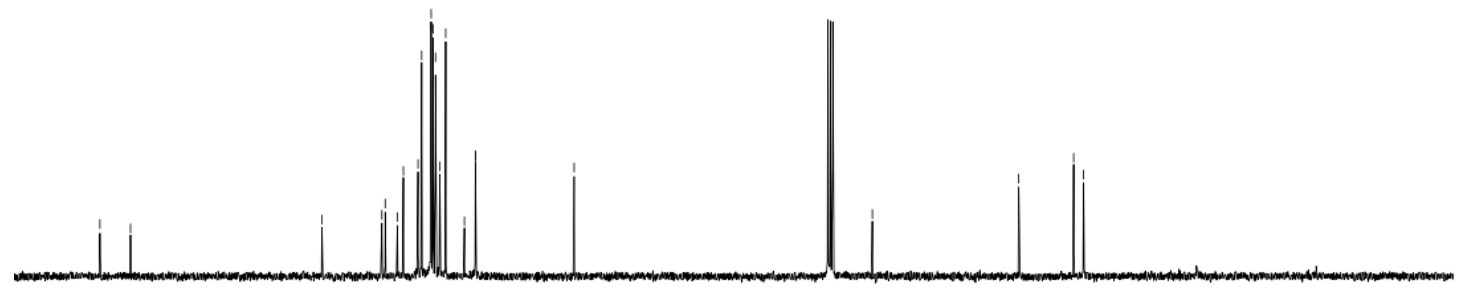

$180 \quad 170$

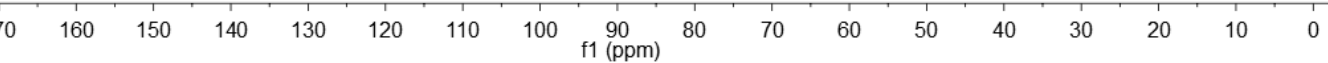

${ }^{1} \mathrm{H}$ and ${ }^{13} \mathrm{C}$ NMR spectra of $\mathbf{6 a d}$ 


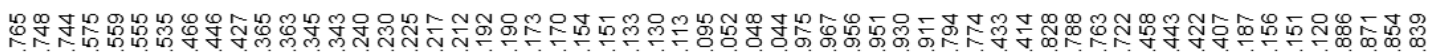

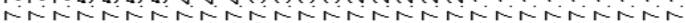<smiles>O=C(O)N(C(=O)c1ccccc1)C1(c2ccccc2)C(=O)Nc2ccccc21</smiles>

6ae Bn

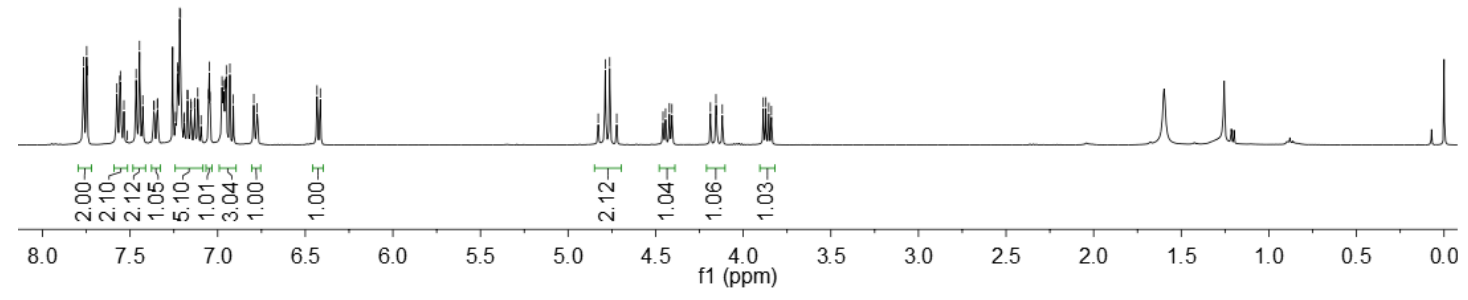

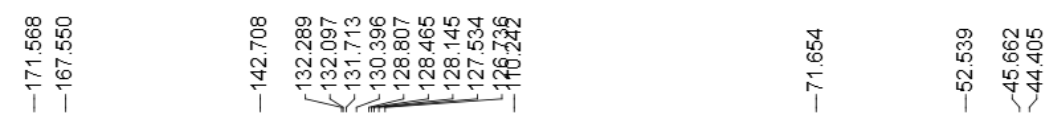

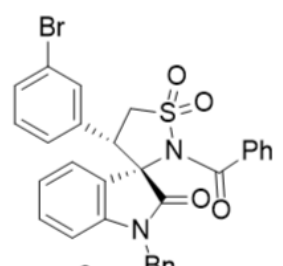

6ae Bn

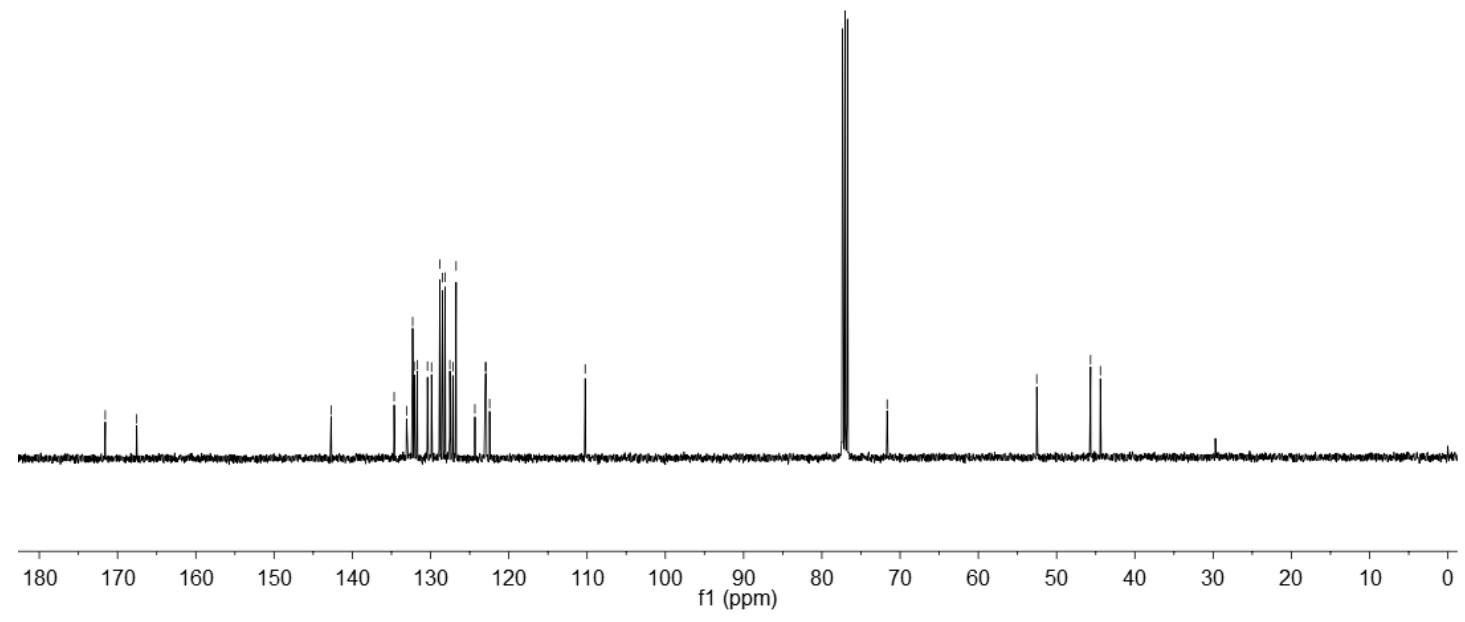

${ }^{1} \mathrm{H}$ and ${ }^{13} \mathrm{C}$ NMR spectra of $\mathbf{6 a e}$ 


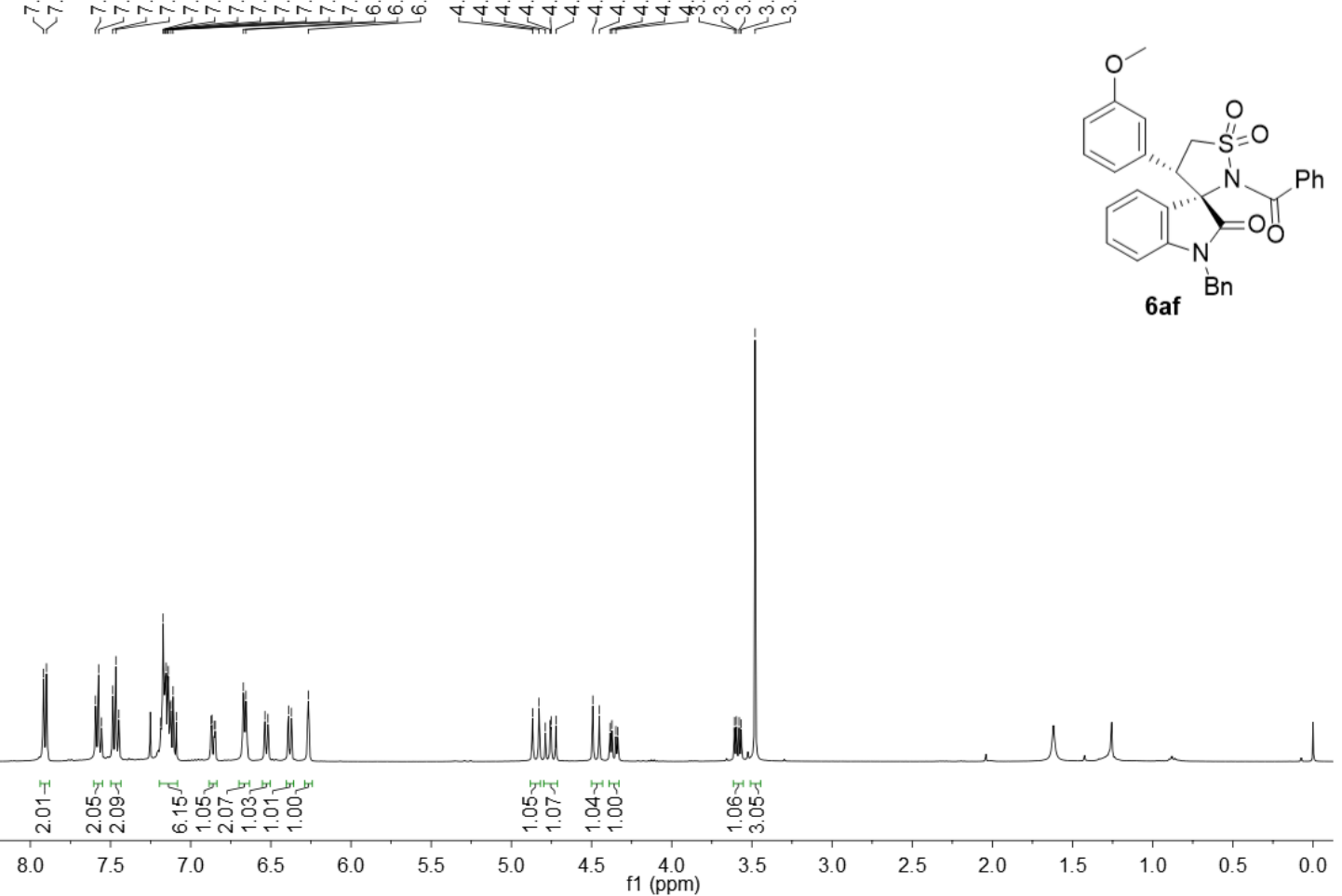

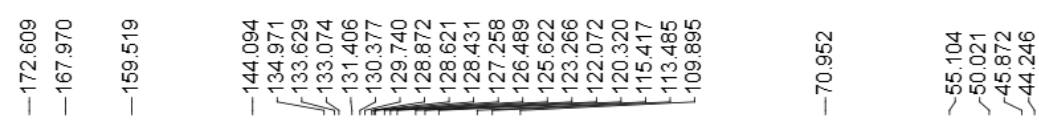
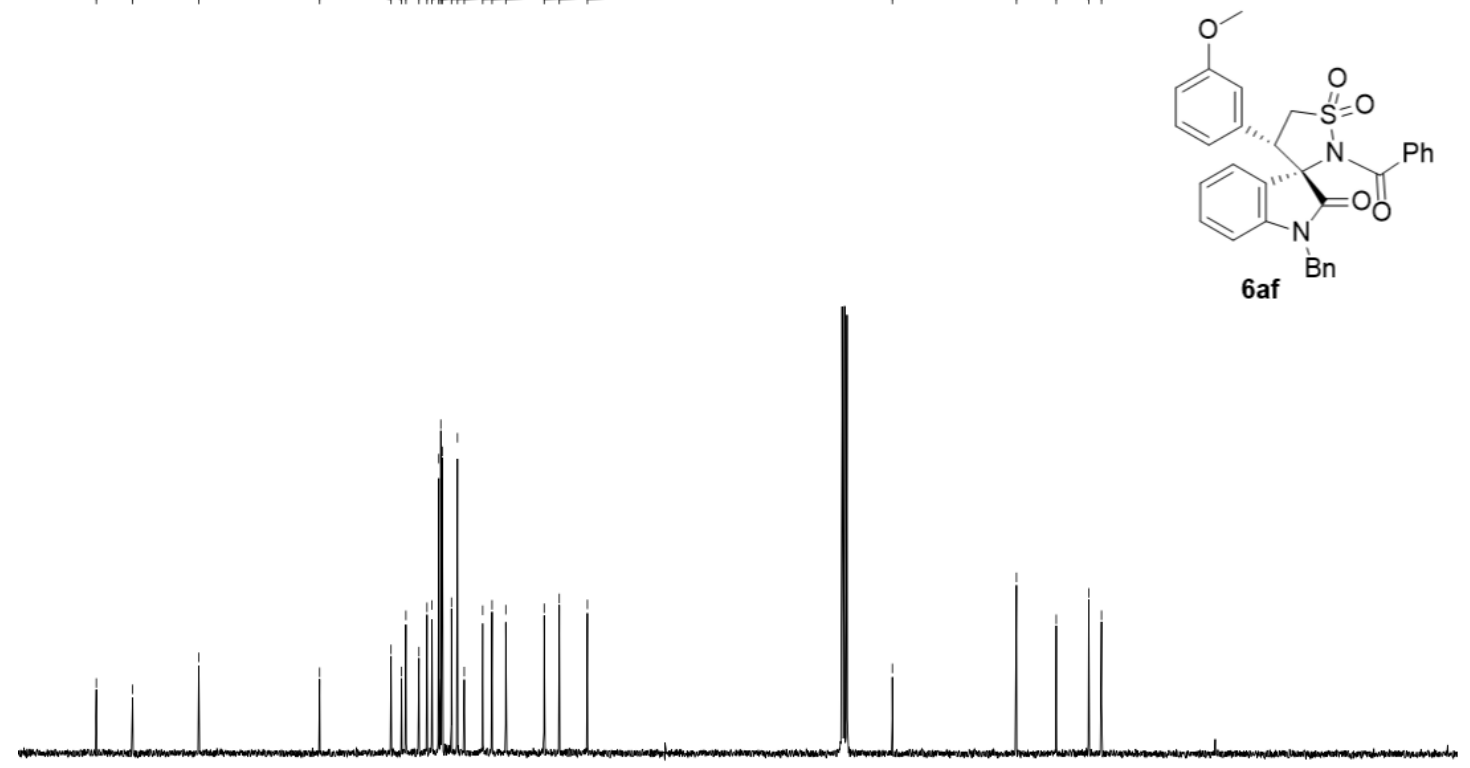

6af $\mathrm{Bn}$

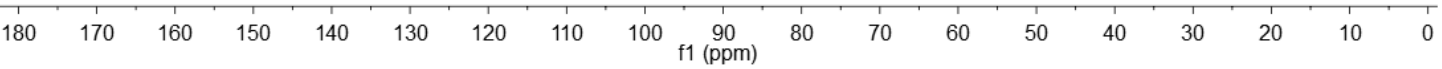

${ }^{1} \mathrm{H}$ and ${ }^{13} \mathrm{C}$ NMR spectra of $\mathbf{6 a f}$ 


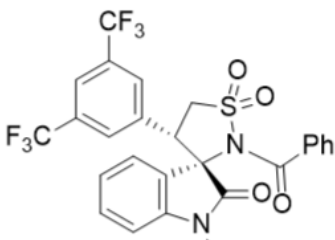

6ag Bn

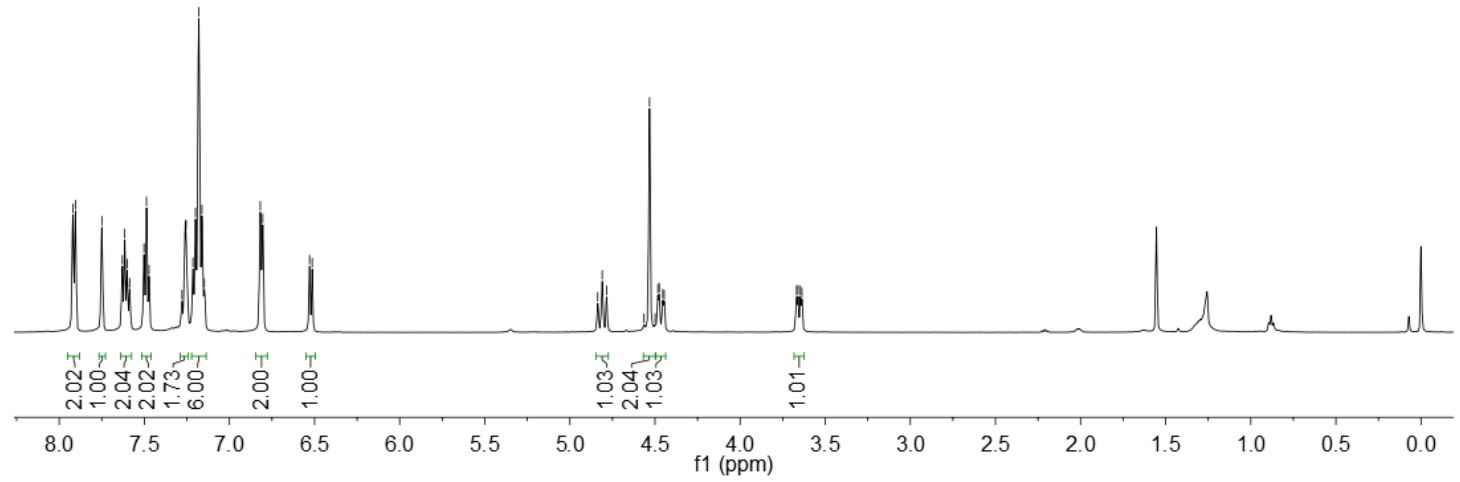

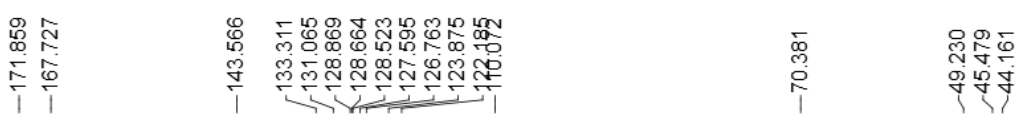

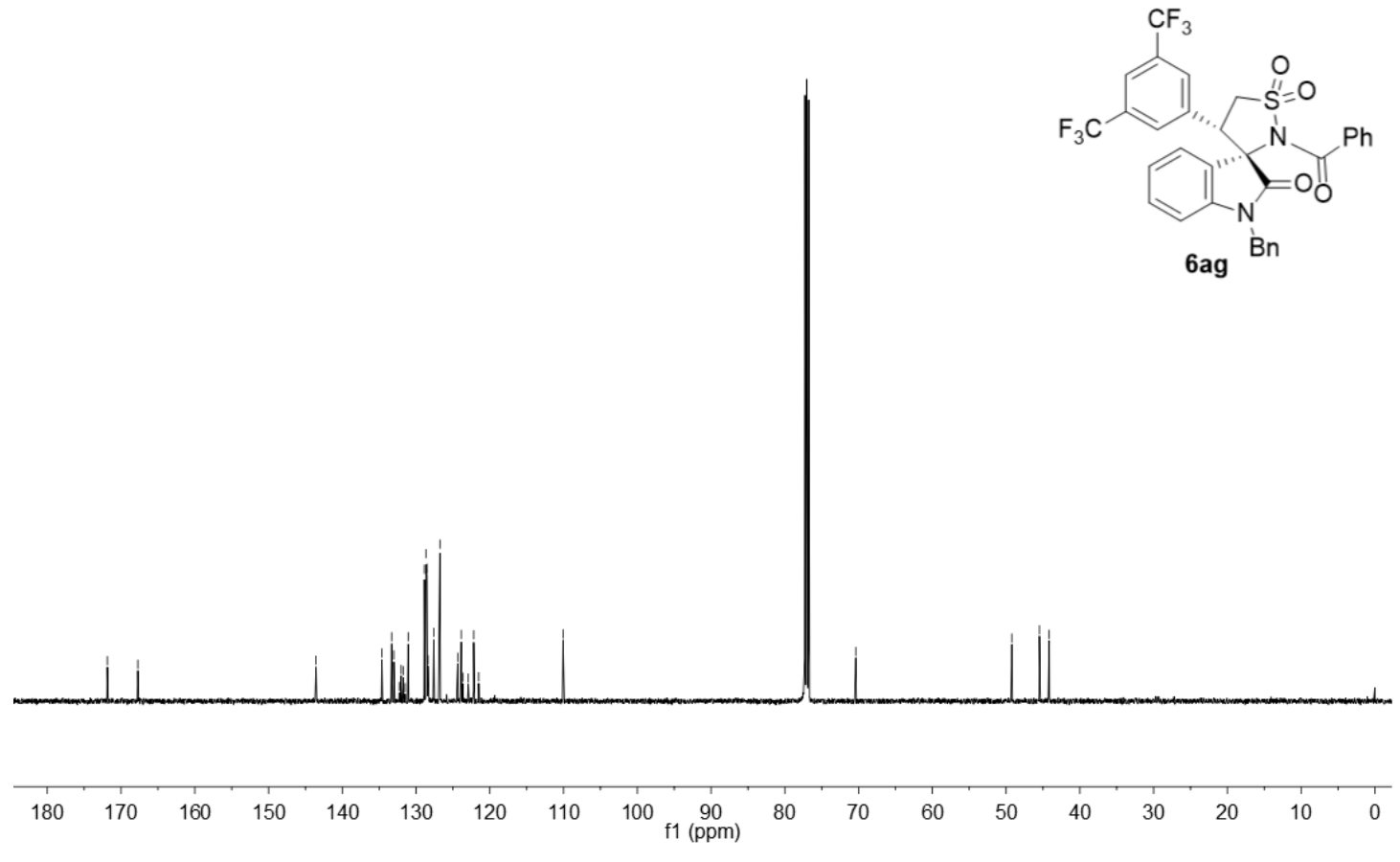

${ }^{1} \mathrm{H}$ and ${ }^{13} \mathrm{C}$ NMR spectra of $\mathbf{6 a g}$ 


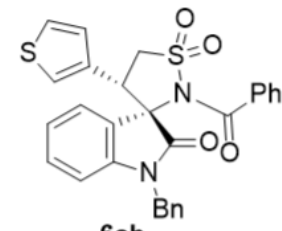

6ah $^{\mathrm{Bn}}$

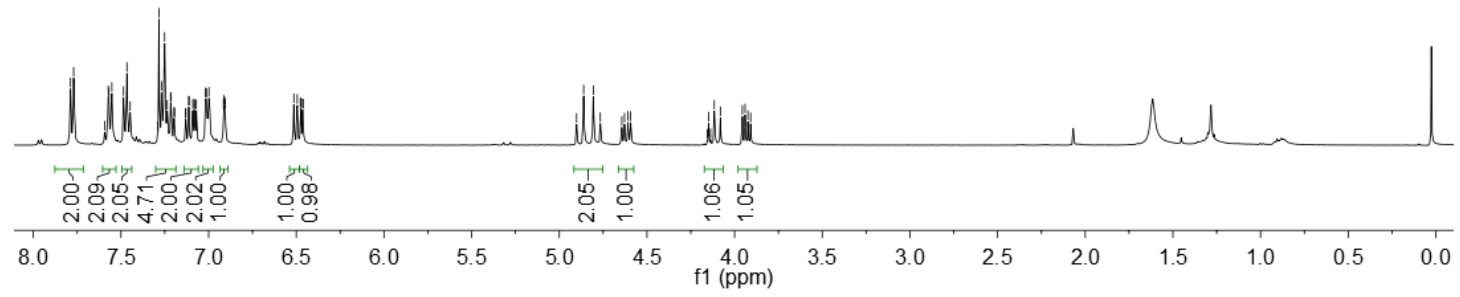

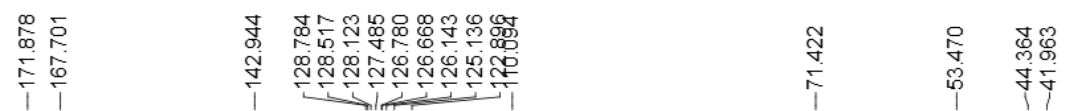

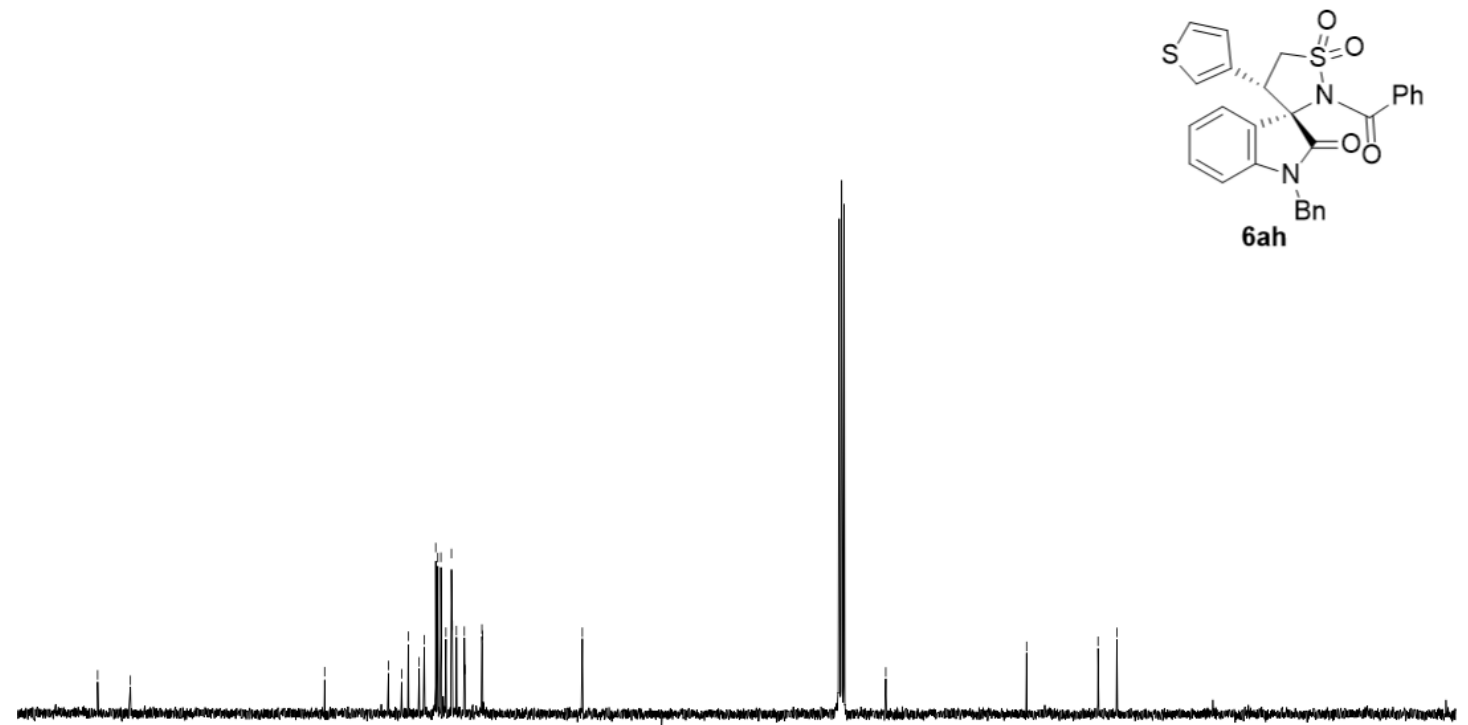

180

$\begin{array}{lllllllll}170 & 160 & 150 & 140 & 130 & 120 & 110 & 100 & \begin{array}{c}90 \\ \mathrm{f} 1(\mathrm{ppm})\end{array}\end{array}$

${ }^{1} \mathrm{H}$ and ${ }^{13} \mathrm{C}$ NMR spectra of $\mathbf{6 a h}$ 


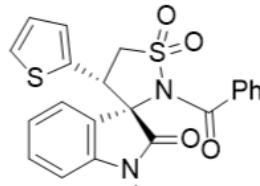

6ai $\mathrm{Bn}$

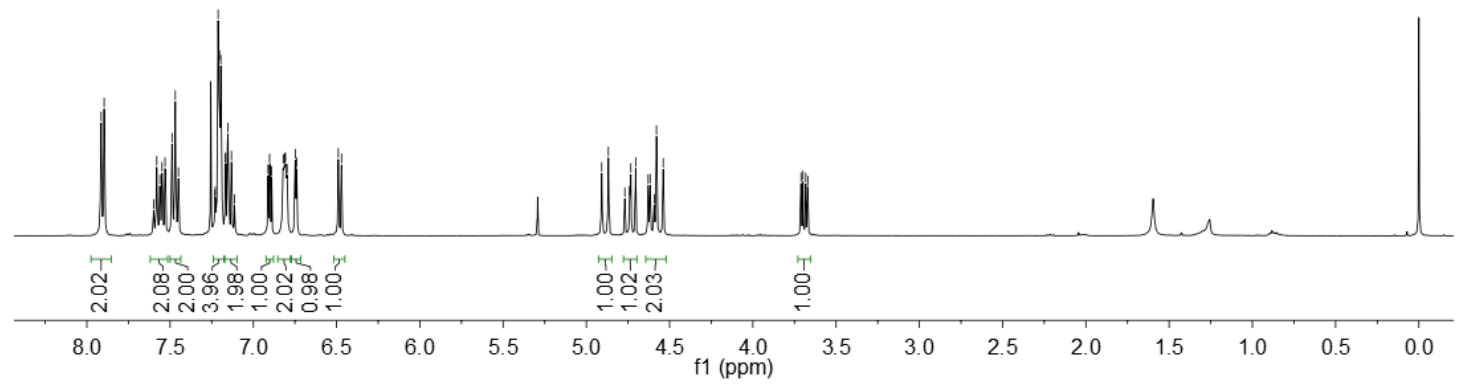

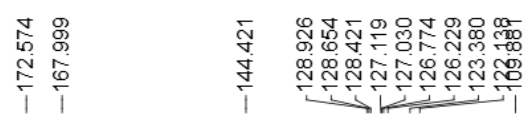

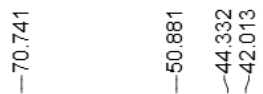

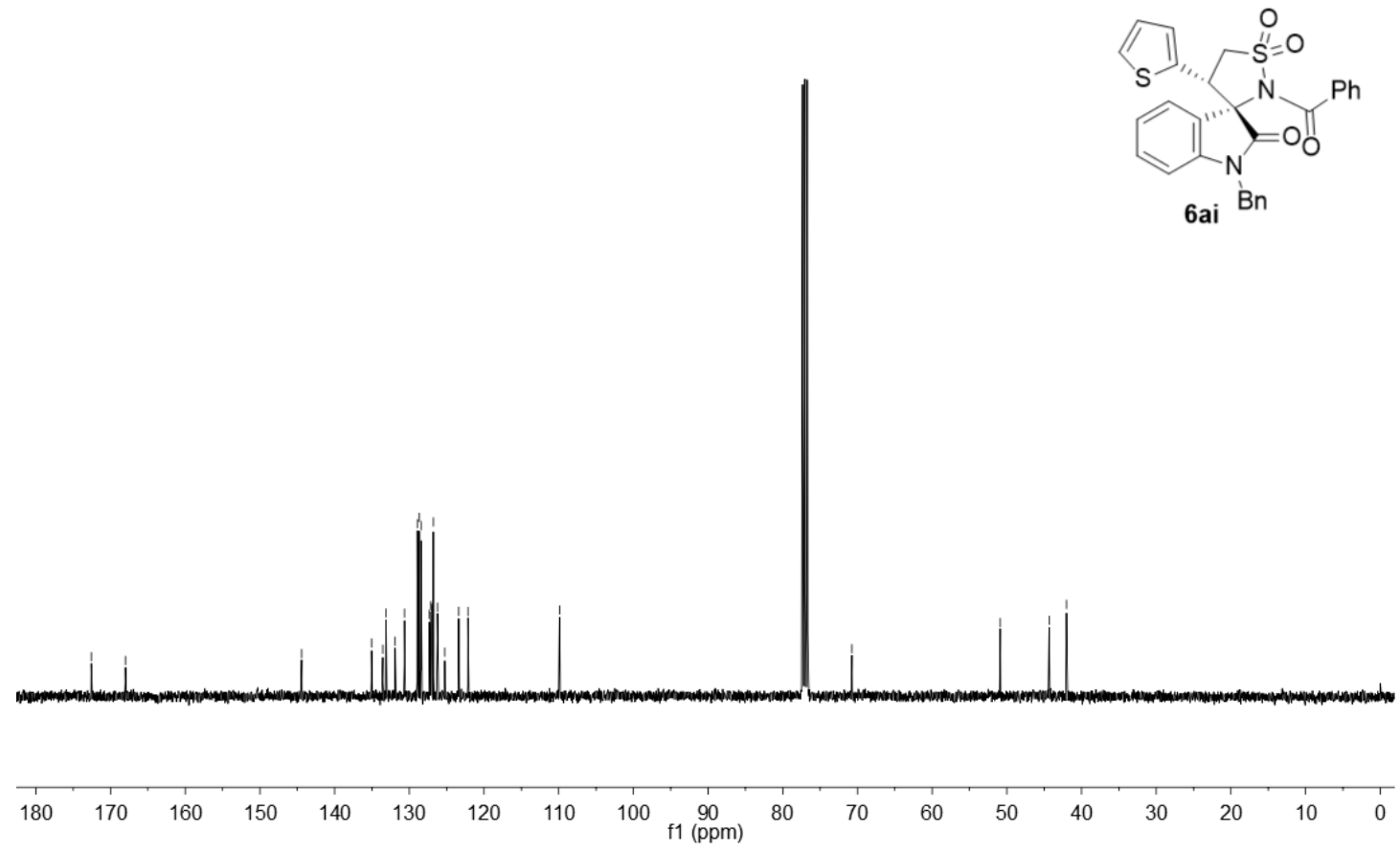

${ }^{1} \mathrm{H}$ and ${ }^{13} \mathrm{C}$ NMR spectra of $\mathbf{6 a i}$ 


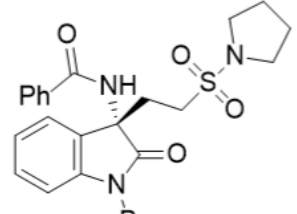

$7 \mathrm{a} \mathrm{Bn}$

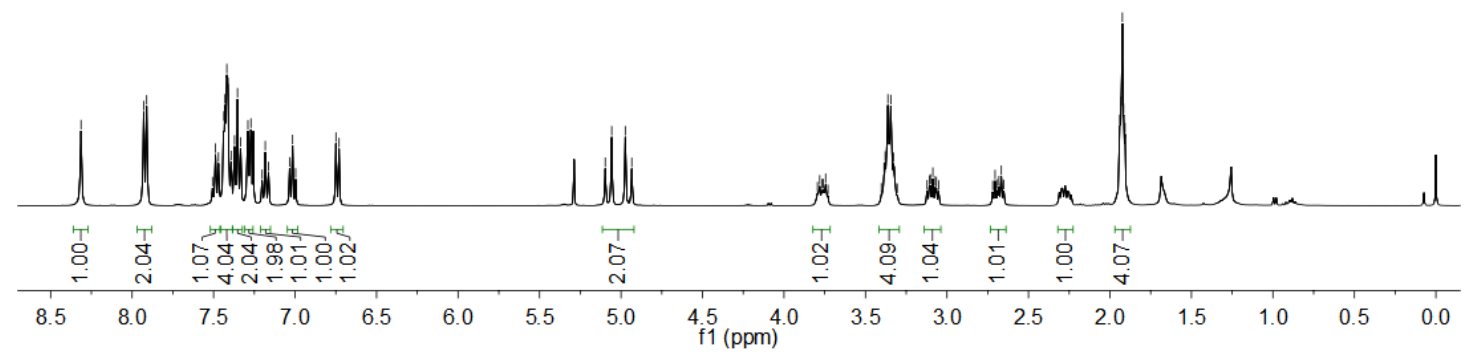

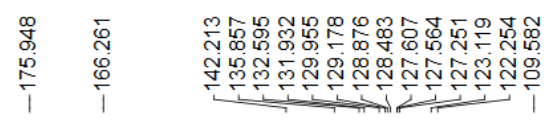

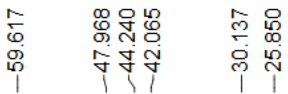

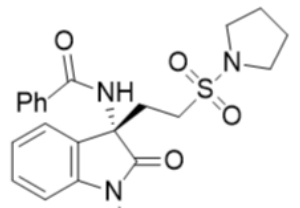

7a $\mathrm{Bn}$

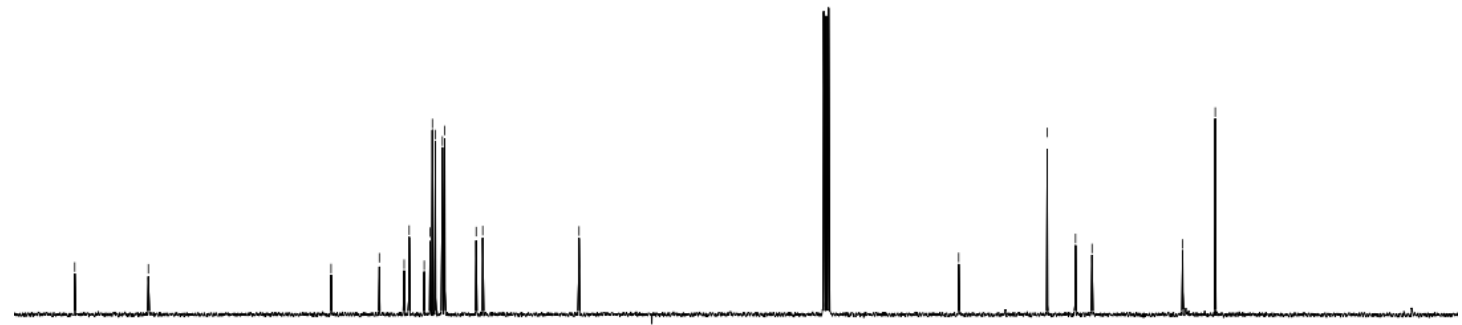

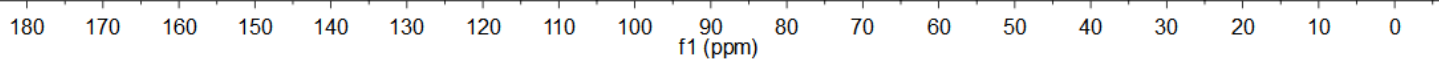

${ }^{1} \mathrm{H}$ and ${ }^{13} \mathrm{C}$ NMR spectra of $\mathbf{7 a}$ 


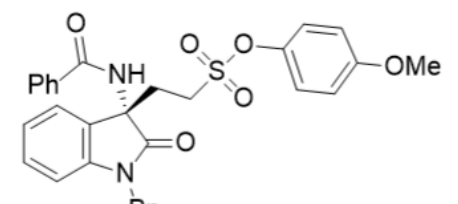

$8 \mathbf{a}$

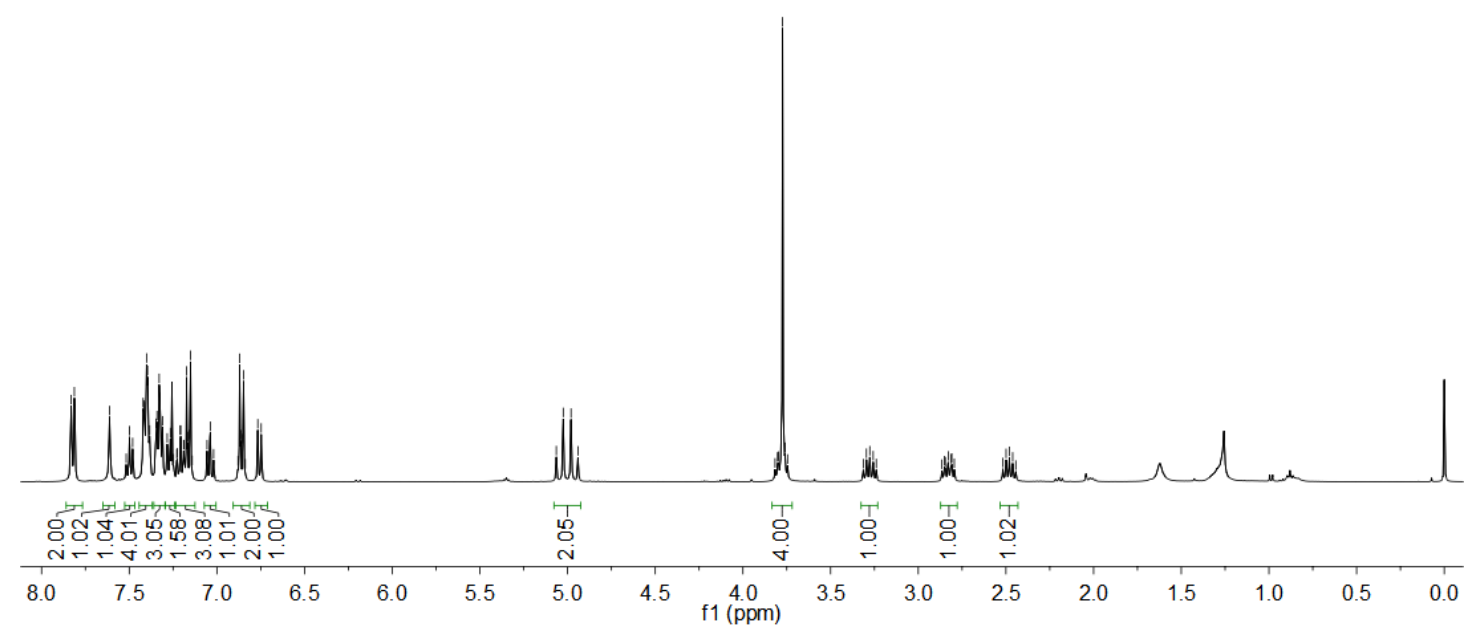

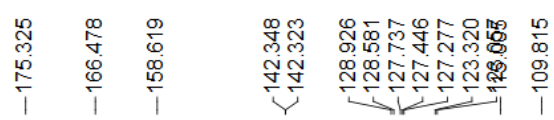

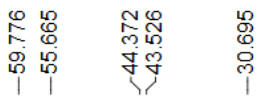

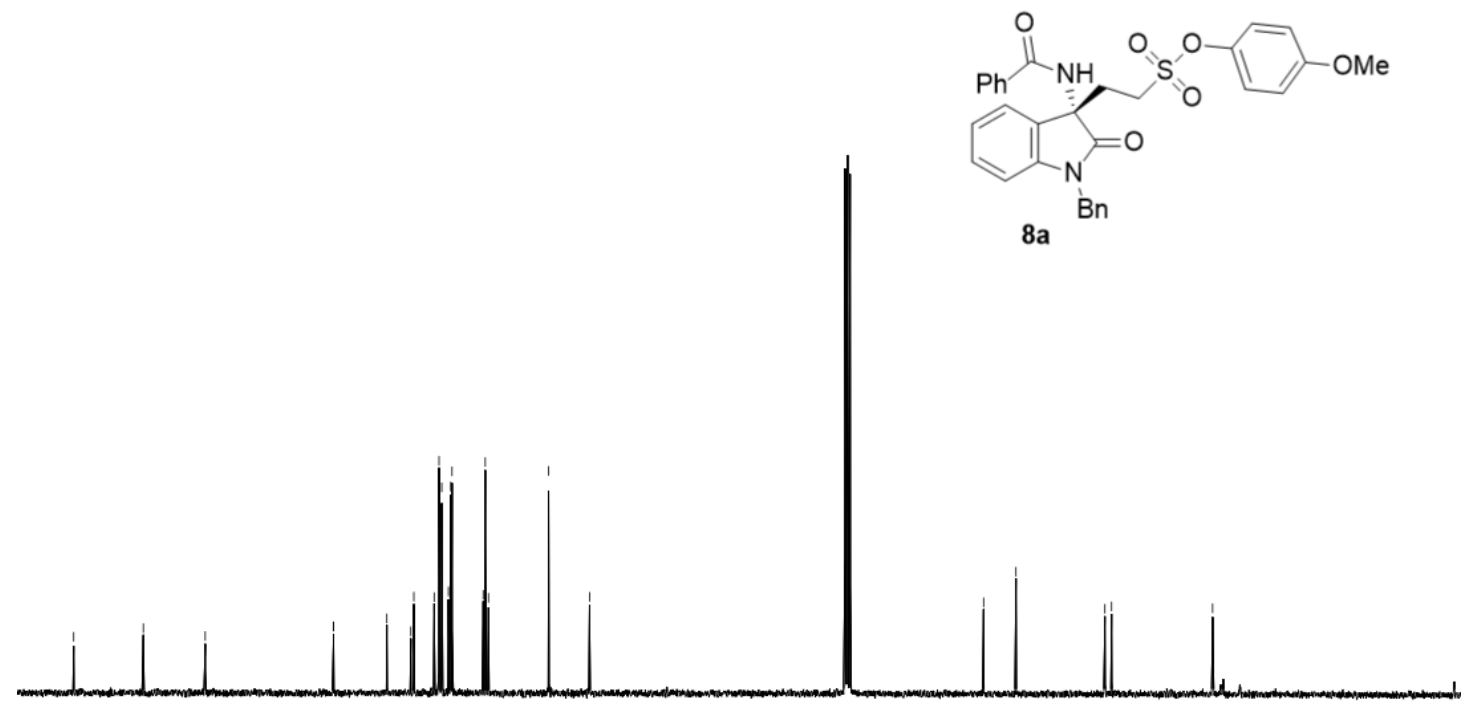

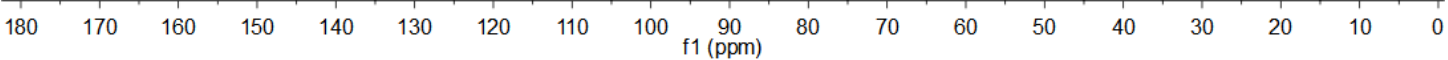

${ }^{1} \mathrm{H}$ and ${ }^{13} \mathrm{C}$ NMR spectra of $\mathbf{8 a}$ 